\title{
MAKING ICU RESEARCH HAPPEN: A QUALITATIVE DESCRIPTIVE STUDY ABOUT THE ROLE OF THE RESEARCH NURSE IN NEW ZEALAND LEVEL III INTENSIVE CARE UNITS
}

\author{
by
}

Diane Margaret Mackle

\begin{abstract}
A thesis
submitted to the Victoria University of Wellington

in fulfilment of the requirements for the degree of

Masters of Nursing
\end{abstract}

Victoria University of Wellington

(2012) 


\section{Abstract}

This study explored the role of the research nurse in New Zealand (NZ) Level III intensive care units (ICU). Little was known about this role in NZ prior to this study. A qualitative, descriptive approach, using semi-structured interviews was used. The study was conducted in six Level III ICUs throughout NZ, who employed a research nurse. Interviews were conducted with research nurses $(\mathrm{n}=11)$, the doctors they work with (principal investigators) $(\mathrm{n}=6)$ and nurse managers $(\mathrm{n}=6)$ for the ICUs, and the findings were triangulated. The views across all ICUs and stakeholders were generally similar, with differences only being in some operational areas. This study found that the primary role of the research nurse was trial management, where they coordinated all elements of trial conduct. Almost half of the research nurses were also involved in trial design through their positions on management committees. Research nurses also played a vital role in patient and trial advocacy, and they bridged the knowledge gap by bringing research to staff nurses, patients and their families. The issue of consent for clinical trials in the ICU setting was significant, as this was a process which research nurses were very involved in. Consenting patients was a shared responsibility of research nurses and doctors. There was a perception that research nurses were senior nurses, but not necessarily because of their role in research. The majority of research nurses reported to a nursing line manager, and also had an informal accountability to the principal investigator (PI). Research nurses and PIs worked closely in the pursuit of rigorous research for ICU patients, and research nurses were highly regarded by PIs. This study provides clarity about the research nurse's role and showcases their key contribution in ensuring that NZ ICUs undertake high quality research, thus contributing to potential improvements for future patients' outcomes.

Keywords: Research nurse, research coordinator, intensive care, role, New Zealand, descriptive methodology 


\section{Acknowledgements}

I would like to acknowledge all of the participants who took time out of their extremely busy work days to welcome me to their ICUs and be interviewed. The research nurses who participated in this study talked about respecting the contribution of patients who participated in ICU studies. I hope I have respected their contribution both in the time and support they have given me throughout this study.

My supervisor, Kathy Nelson has been a huge source of expertise and support throughout the writing of this thesis. I have always felt like she was 'on my side' and her advice and mentoring in all matters has been invaluable. I cannot thank you enough.

I would like to acknowledge the huge contribution my husband Steve has made to this thesis. He has kept our three children fed, clothed and entertained for the last two years so that I could devote time to my thesis. I could not have done this without him. To my children; Ruby, Eva and Luke, I hope you appreciate our efforts in the future.

Many people inspired and supported me during the writing of this thesis - I would like to extend my thanks to all of you. Special mention must go to my friend and colleague, Elinore Harper who was always 'one step ahead of me' writing her thesis. I value the support and advice you have given me, to make my journey an easier one. Dr Peter Hicks has always supported me in gathering and analysing quantitative data, and gave me an insight into the College of Intensive Care Medicine guiding documents. Dr Paul Young has shown me what hard work and a desire to conduct clinical research can achieve in a short space of time.

This type of study would be extremely difficult to complete without financial support. I would like to thank Victoria University of Wellington for the grants which paid for travelling to locations around New Zealand to interview the participants, and then to assist with transcribing the interviews - this came at just the right time. I would like to thank the Clinical Training Agency (CTA) which has contributed to my university enrolment fees. Without this type of support, conducting Masters-level studies would be beyond the reach of many nurses. I would also like to thank my Charge Nurse Manager, Steve James who encouraged me to do this research, supported me with study days and for endorsing my 
application to the CTA. This study would not have happened if Professor Jo Walton had not supported it - thank you for allowing me to research a topic I am passionate about.

The librarians at both Victoria University of Wellington library and University of Otago Medical School library have been outstanding and have assisted me with locating books and articles, beyond my expectations. 


\section{Table of contents}

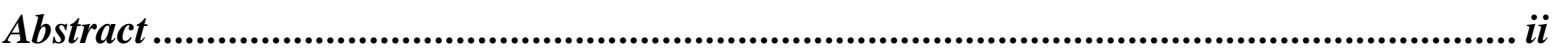

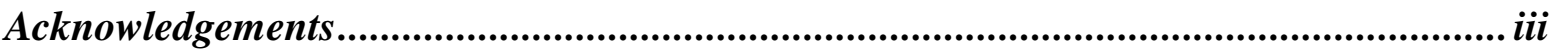

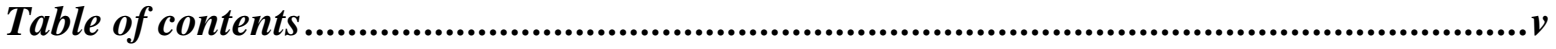

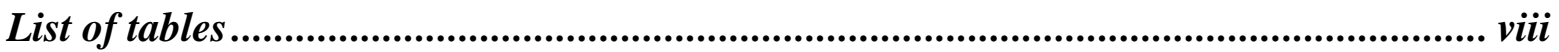

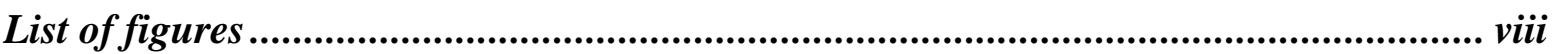

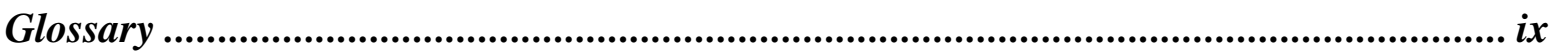

Chapter 1 Introduction................................................................................. 1

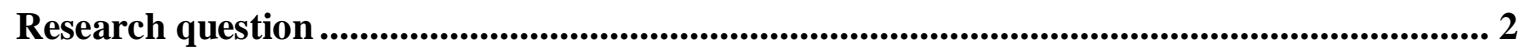

Aims and objectives of this study ............................................................................................... 2

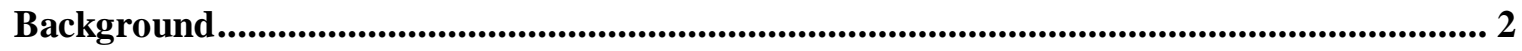

Formulating the research question............................................................................................................... 4

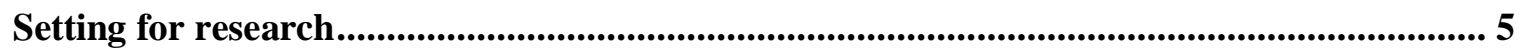

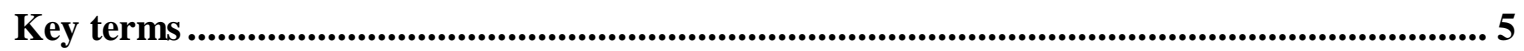

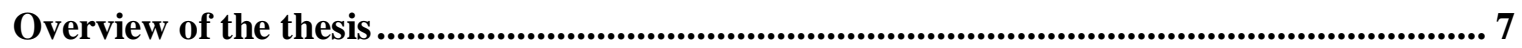

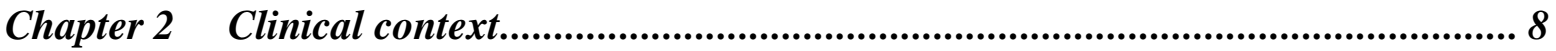

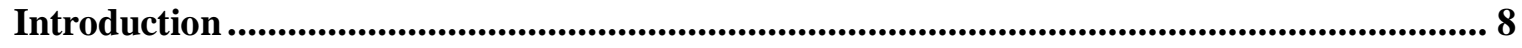

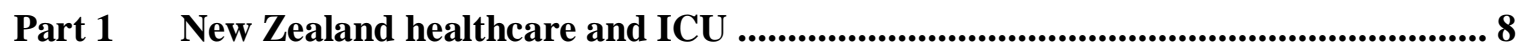

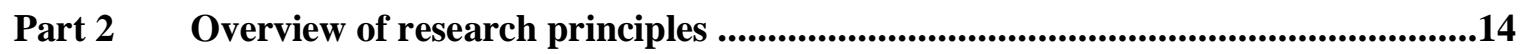

Part 3 Bringing two specialties together - research in the ICU setting ............................18

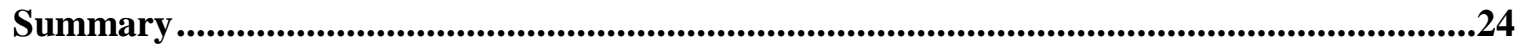

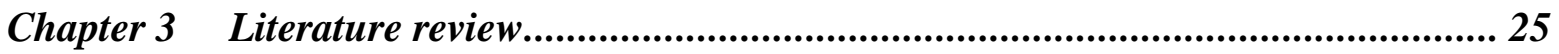

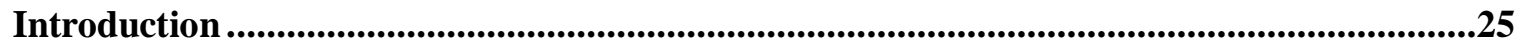

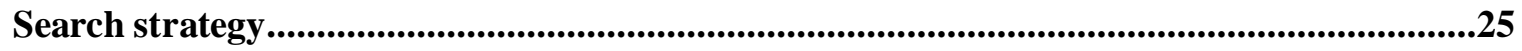

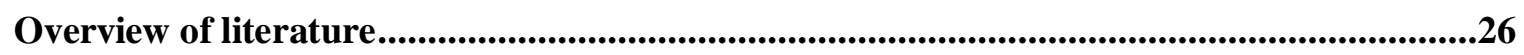

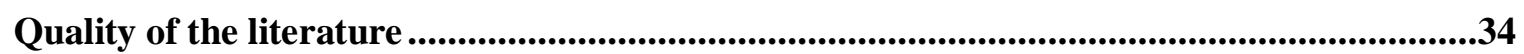

Development of the research nurse role ..............................................................................................34

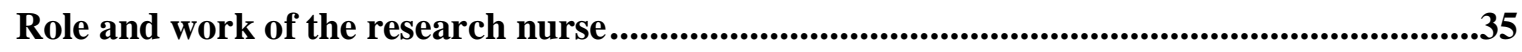


Ethics, patient advocacy and consent ..............................................................................................40

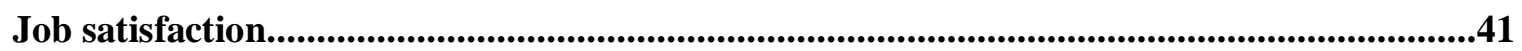

Role preparedness, career pathways and professional development ......................................44

Summary and areas for further research ...........................................................................................46

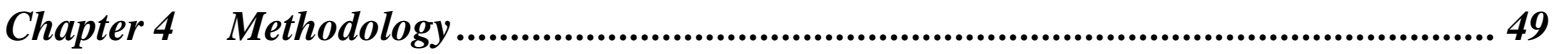

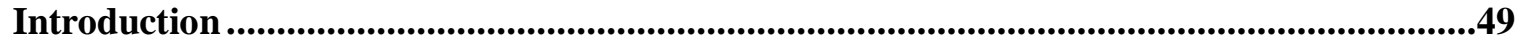

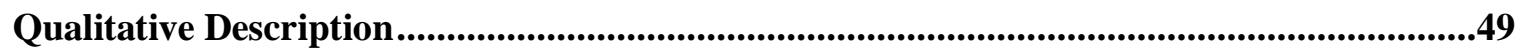

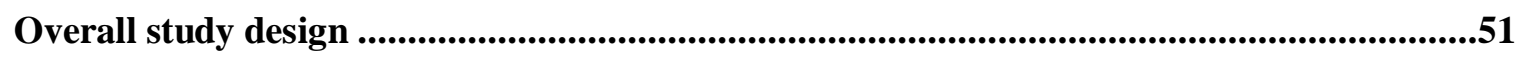

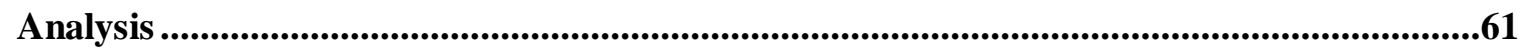

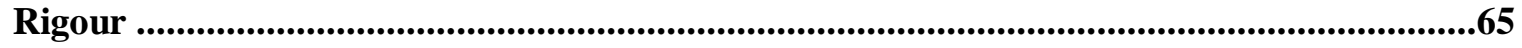

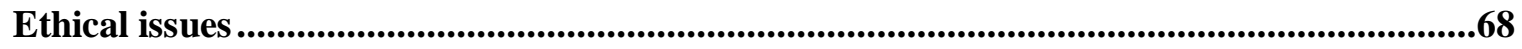

Summary

Chapter 5 Findings: Research participants, departments and supporting structures... 71

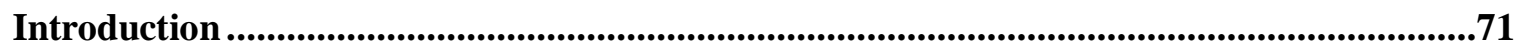

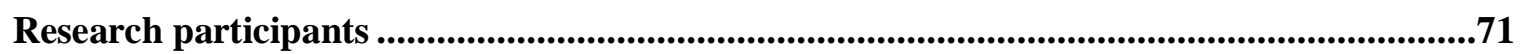

Research departments - structure and funding ............................................................................73

Research nurses .........................................................................................................................83

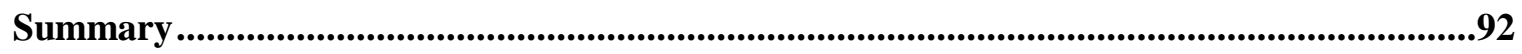

Chapter 6 Findings: Research nurse role ................................................................ 93

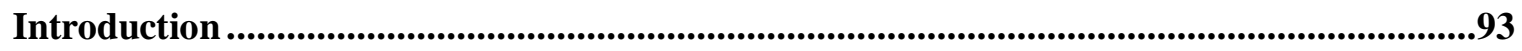

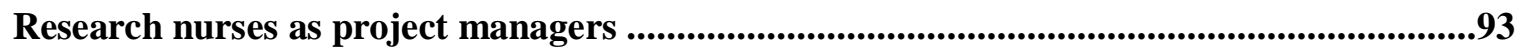

Research nurses balancing patient needs with research requirements ..................................104

Research nurses bridging gaps and making research happen................................................113

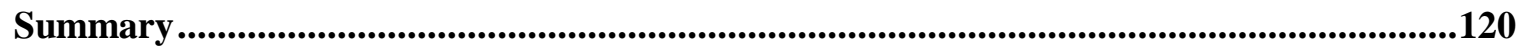

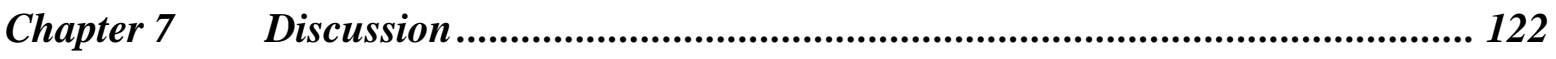

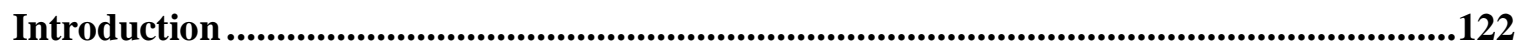

Overview of findings ......................................................................................................................122

Trial management - the research nurse's primary role.................................................123 
Patient advocacy .......................................................................................................................................126

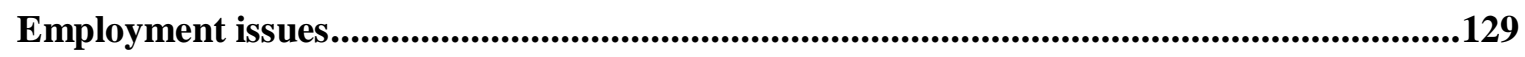

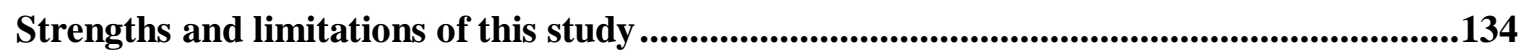

Reflection on methods of inquiry .......................................................................................................134

Recommendations .................................................................................................................138

Conclusion...............................................................................................................................................140

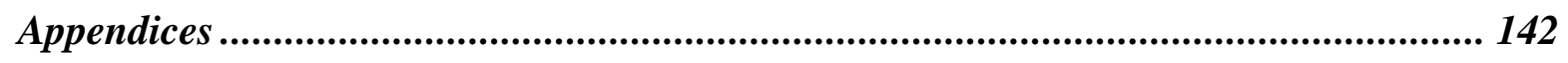

Appendix A Summary of quality of literature using STROBE criteria...........................142

Appendix B Interview schedule - research nurses .....................................................145

Appendix C Interview schedule - principal investigators ...................................................151

Appendix D Interview schedule - nurse managers ..............................................................155

Appendix E Information sheet for research nurses ..............................................................160

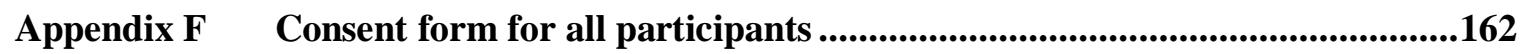

Appendix G Information sheet for principal investigators and nurse managers .............163

Appendix H Transcriber agreement ...................................................................................165

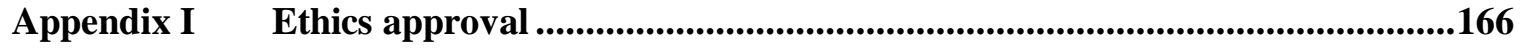

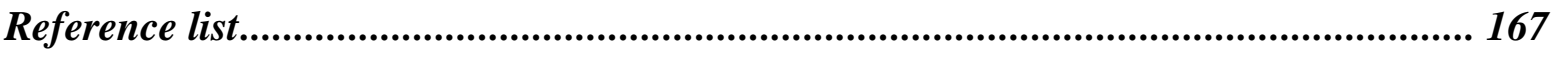




\section{List of tables}

Table 1 Requirements for the 3 levels of Intensive Care Unit in NZ .......................... 10

Table 2 Summary of New Zealand Intensive Care Units by level.............................. 13

Table 3 Types of consent used in the ICU setting ................................................ 20

Table 4 Summary of relevant literature about the role of research nurses ................... 28

Table 5 Levels of responsibility in responsibility assignment matrix........................ 56

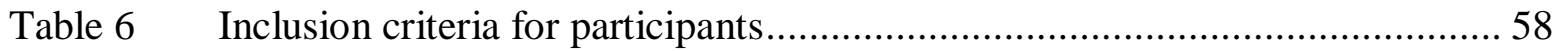

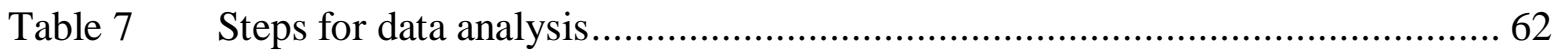

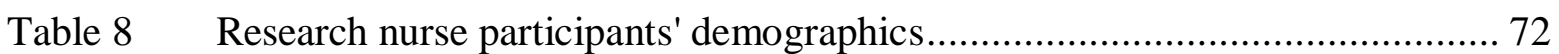

Table 9 Summary of principal investigators and studies for each ICU....................... 76

Table 10 Summary of ICUs' funding streams, research nurse FTE and overheads ........ 77

Table 11 On call and call back payments for ICU research nurses by ICU .................. 89

Table 12 Research nurses' role in the consent process ............................................ 108

\section{List of figures}

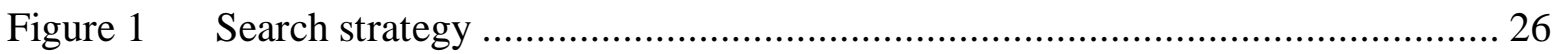

Figure 2 Sample responsibility assignment matrix used for this study ....................... 57

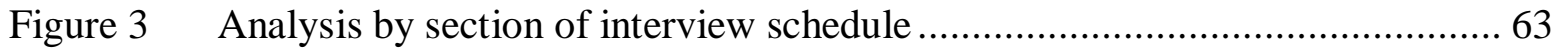

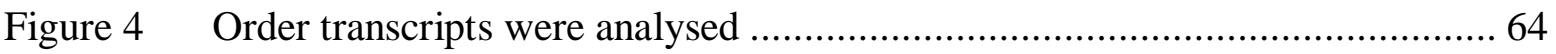

Figure 5 Sample of analysis using Responsibility Assignment Matrix .......................... 65

Figure 6 Reported line management models for research nurses.............................. 74

Figure 7 Responsibility assignment matrix - selecting studies for ICU ..................... 96

Figure 8 Responsibility assignment matrix - trial set up stage (selected tasks) ............ 99

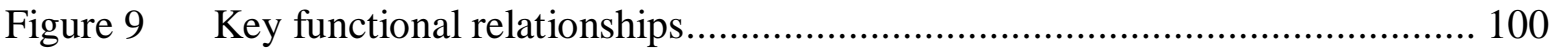

Figure 10 Responsibility Assignment Matrix - consent process (lower risk studies) ..... 109

Figure 11 Responsibility Assignment Matrix - consent process (higher risk studies) .... 111 


\section{Glossary}

\begin{tabular}{|c|c|}
\hline $\begin{array}{l}\text { Associate Charge Nurse } \\
\text { Manager (ACNM) }\end{array}$ & $\begin{array}{l}\text { Senior nurse who provides clinical coordination and } \\
\text { leadership, and manages resources for a shift. Also called } \\
\text { 'charge nurse'. Reports to the nurse manager. }{ }^{1}\end{array}$ \\
\hline Adverse Event & $\begin{array}{l}\text { Any untoward event or symptom either related to or not } \\
\text { related to the study treatment. }{ }^{2}\end{array}$ \\
\hline ANZICS & $\begin{array}{l}\text { Australia and New Zealand Intensive Care Society. A } \\
\text { professional body for intensivists }{ }^{3} \text {. }\end{array}$ \\
\hline Blinded & $\begin{array}{l}\text { Where one or more parties are kept unaware of whether a } \\
\text { subject is on a study treatment or control arm. }\end{array}$ \\
\hline Case Report Form (CRF) & $\begin{array}{l}\text { An electronic or paper form to record all information, } \\
\text { including data for a study subject. }{ }^{4}\end{array}$ \\
\hline Central Venous Line (CVL) & An intravenous catheter which is inserted into a large vein. \\
\hline CICM & $\begin{array}{l}\text { College of Intensive Care Medicine. }{ }^{5} \text { The body which } \\
\text { provides training, education and professional development } \\
\text { for intensivists. }\end{array}$ \\
\hline Clinical Director & Senior medical officer in charge of the ICU. \\
\hline Competence & $\begin{array}{l}\text { "A legal term used to denote capacity to act on one's own } \\
\text { behalf, the ability to understand information presented, to } \\
\text { appreciate the consequences of acting (or not acting) on that } \\
\text { information, and to make a choice." (p. 99) }\end{array}$ \\
\hline CTG & $\begin{array}{l}\text { ANZICS Clinical Trials Group. A collaborative group of } \\
\text { intensivists and researchers committed to conducting high } \\
\text { quality research in ICU. }\end{array}$ \\
\hline $\begin{array}{l}\text { Full Time Equivalence } \\
\text { (FTE) }\end{array}$ & $\begin{array}{l}\text { The proportion of a full-time ( } 40 \text { hour) working week. } 1.0 \\
\text { FTE is full-time. }\end{array}$ \\
\hline GCP & $\begin{array}{l}\text { Good clinical practice (also called 'Good clinical research } \\
\text { practice' and International Conference on Harmonisation } \\
\text { good clinical practice [ICH-GCP]). An international standard } \\
\text { for the ethical conduct of clinical research. }{ }^{4}\end{array}$ \\
\hline HDEC & New Zealand Health and Disability Ethics Committee ${ }^{2}$ \\
\hline $\begin{array}{l}\text { Incompetence (or } \\
\text { incapacity) }\end{array}$ & $\begin{array}{l}\text { A legal term meaning an inability to manage one's own } \\
\text { affairs }{ }^{2} \text {. Patients in ICU are often termed 'incompetent' due } \\
\text { to their severity of illness, and level of consciousness } \\
\text { (resulting from their illness, or induced through to sedation) }\end{array}$ \\
\hline Nurse Manager (NM) & $\begin{array}{l}\text { Clinical leader for nurses. Has overall responsibility for ICU } \\
\text { nursing staff and provides professional leadership to nursing } \\
\text { staff. Manages systems, processes and resources. }{ }_{1}\end{array}$ \\
\hline Monitor & $\begin{array}{l}\text { Also called 'Study monitor'. The person who oversees the } \\
\text { conduct of a trial, and ensures that it complies with GCP } \\
\text { guidelines. }\end{array}$ \\
\hline
\end{tabular}




\begin{tabular}{|c|c|}
\hline NZNO & $\begin{array}{l}\text { New Zealand Nurses Organisation. A professional body and } \\
\text { union for nurses in NZ. }\end{array}$ \\
\hline Protocol & $\begin{array}{l}\text { Also called study protocol. "A document that describes the } \\
\text { objective(s), design, methodology, statistical considerations, } \\
\text { and organization [sic] of a trial" (p.10). }{ }^{4}\end{array}$ \\
\hline $\begin{array}{l}\text { Protocol violation or } \\
\text { deviation }\end{array}$ & An incident where the protocol is not adhered to. \\
\hline RAM & Responsibility Assignment Matrix ${ }^{8}$ \\
\hline Reportable event & $\begin{array}{l}\text { Any adverse event which puts a patient or staff member at } \\
\text { risk, or has the potential to do so. }\end{array}$ \\
\hline Serious Adverse Event & $\begin{array}{l}\text { "Any untoward medical occurrence that at any dose; results } \\
\text { in death, is life-threatening, required inpatient hospitalization } \\
\text { [sic] or prolongation of existing hospitalization, results in } \\
\text { persistent or significant disability/incapacity or is a } \\
\text { congenital anomaly/birth defect" (p. } 9-10){ }^{4}\end{array}$ \\
\hline Screening log & $\begin{array}{l}\text { The log which is kept and reported to the sponsor, of all } \\
\text { patients who were considered for a clinical trial, but were } \\
\text { excluded. }\end{array}$ \\
\hline Sponsor & $\begin{array}{l}\text { "An individual, company, institution or organization which } \\
\text { takes responsibility for the initiation, management, and/or } \\
\text { financing of a clinical trial" (p. 10). }{ }^{4}\end{array}$ \\
\hline Subinvestigator & $\begin{array}{l}\text { "Any individual member of the of the clinical trial team } \\
\text { designated and supervised by the investigator at a trial site to } \\
\text { perform critical trial-related procedures and/or make } \\
\text { important trial-related decisions" (p. 10). }\end{array}$ \\
\hline
\end{tabular}

Adapted from:

1 District Health Boards/New Zealand Nurses Organisation. (2012). District Health Boards/New Zealand Nurses Organisation Multi-employer collective agreement 2011 - 2015. Retrieved 23 July, 2012, from http://www.nzno.org.nz/dhb

2 Ministry of Health. (2006). Operational standard for ethics committees. Retrieved 10 November, 2011, from http://www.ethicscommittees.health.govt.nz/moh.nsf/pagescm/6777/\$File/OperationalStandard2006.p df

3 Australian and New Zealand Intensive Care Society. (2010). 'About us' webpage. Retrieved 30 November, 2010, from http://www.anzics.com.au/about-us

$4 \quad$ European Medicines Agency. (2002). Note for guidance on good clinical practice Step 5. Retrieved 10 May, 2012, from http://www.ema.europa.eu/docs/en_GB/document_library/Scientific_guideline/2009/09/WC5000028 74.pdf

5 College of Intensive Care Medicine. (2012). 'About us' webpage. Retrieved 12 June, 2012, from http://www.cicm.org.au/aboutus.php

$6 \quad$ Clinical Trials Group. (2010). CTG website. Retrieved 30 November, 2010, from http://www.anzics.com.au/clinical-trials-group

7 New Zealand Nurses Organisation (2009) Home page. Retrieved 29 July, 2012, from http://www.nzno.org.nz/

8 Project Management Institute. (2008). A guide to the project management body of knowledge (PMBOK guide). PA: Project Management Institute. 


\section{Chapter 1 Introduction}

Intensive care is an environment in which highly skilled healthcare professionals work together to provide high quality care to critically ill patients, to give them the best chance of survival, long-term quality of life, or a dignified death. Intensive care research has the capacity to improve patient-centred outcomes and quality of life for future intensive care unit (ICU) patients. Conducting research in ICU is challenging, as the patients are amongst the sickest and most vulnerable in the healthcare system. Research nurses play a key role in overcoming these challenges and 'making research happen' in the ICU. This thesis reports on a study that explores the role of research nurses in New Zealand (NZ) ICUs.

This chapter introduces the research question, aims and objectives for the study. The background about the impetus for this study is provided, then how the research question was formulated. The setting and design is then outlined, key terms are introduced and an overview of the thesis is given.

This study is important because many ICUs in NZ employ research nurses to assist in conducting clinical research trials. However, little is known about the details of the role and work of research nurses and their contribution to ICU patient care in NZ. While there is international data about the role in general, much of it has been undertaken in populations with less acutely unwell participants. Studies set in Australian ICUs included NZ respondents; however, NZ has been grouped with Australia in analysing the role. This study will add to a growing body of knowledge about the role of the research nurse, and describe the role in an acute clinical setting. Describing what research nurses do, will also be a first step to understanding the role, enabling future development of the role including career pathways and professional opportunities.

Research nurses have various titles including research nurse, clinical research nurse, research coordinator and specialty nurse research. But do they share the same role and do they all do the same work? What is that work, and what is important about the work they do? This study sought to examine the role of the research nurse in the research process, and was designed to examine the role from the perspective of the research nurse themselves, the doctor they work closely with (principal investigator) and their nurse manager. 


\title{
Research question
}

The research question for this thesis is "What is the role of the Research Nurse in New Zealand Level III Intensive Care Units?'

\section{Aims and objectives of this study}

\begin{abstract}
Aims
This study aims primarily to describe the role and responsibilities of the ICU research nurse. This will enable increased understanding of the role and the contribution research nurses make to research and patient care in ICU. A secondary aim is to describe the structures and funding arrangements of the research departments the research nurses work in. This study does not aim to assess whether one version of the role is better than another but describes the role from different perspectives, thus allowing the reader to decide if the role within a particular ICU aligns to their beliefs.
\end{abstract}

\section{Objectives}

The objectives were to gather data about the research nurse role from three different participant groups; Research Nurses, Principal Investigators and Nurse Managers, by way of semi-structured interviews.

It was expected that this research would:

- Describe the demographics of research nurses currently working in NZ ICUs

- Identify important components of the role, the work they do and how different this was across NZ

Secondary objectives were to:

- Identify the structure of the individual ICU research departments

- Identify key relationships pertinent to the research nurse role

- Examine the similarities and differences about the role between professional groups

- Examine the similarities and differences about the role within ICUs.

\section{Background}

ICU is a clinical area I know well, having worked as a staff nurse, clinical nurse coordinator (equivalent to associate charge nurse manager [ACNM]) and since 2007 as a research nurse. As an ICU research nurse working in a Level III NZ ICU, I am an insider in this study. The implications of this to the study are discussed in Chapter 4. The majority of my work involves managing clinical trials and I also advise and assist nurses and doctors with their 
research and audit projects. There has been someone assisting with research in this ICU since the 1990s. Initially this was an ICU technician (F. Fitzjohn, personal communication, $26^{\text {th }}$ April, 2012) then from 2003 until 2006, research was managed by a staff nurse seconded part-time from ICU clinical work. The position of 'Clinical Research Nurse' was first formally established in July 2006 and in 2008, the role title was changed to 'Specialty Nurse Research' and the current position was established. The role was recognised as a designated senior nurse position at the same time (District Health Boards/New Zealand Nurses Organisation [DHB/NZNO], 2012 ).

When I began working as a research nurse, I had little orientation and learned by 'doing'. I drew on my experience in ICU, my academic background and any professional development opportunities that came my way. I turned to principal investigators (PIs), previous research nurses and study monitors for guidance and gradually built my skill level and understanding. Throughout this time I had a growing awareness that this role was not well understood or highly regarded. There seemed to be an imbalance between the high level of responsibility and a lack of recognition and understanding about the role. I attended a study day at the hospital I work in, where one talk unfavourably compared research nurses with 'nurse researchers' who conduct their own nursing research. The speaker implied that research nurses were basically 'doing a job', were 'doing doctor's research', didn't care about patient outcomes and were very poorly thought of by academic nurses. I already had doubts about how research was viewed within my own hospital and ICU because, despite support from the ICU Charge Nurse Manager, no space was allocated for research nurses in a newly planned ICU. Research nurses from all clinical areas were to work in a research department located far from ICU. While suitable for many clinical areas, I thought this showed lack of understanding about ICU research, which operates 24 hours a day, seven days a week, and is very 'hands on'.

These issues made me question whether other ICU research nurses around NZ did the same work as I did, in the same way and faced the same challenges. However, there was little evidence about the work of the ICU research nurse and none specifically about NZ. The literature review by Bell (2009) had reaffirmed that it was a nursing role by aligning it to the Nursing Council of NZ competencies, thus paving the way for future research into research nurses in NZ. I attended two presentations by Leonie Walker, a researcher from the New Zealand Nurses Organisation (NZNO), who highlighted the lack of job security experienced 
by many research nurses, citing her experience in the United Kingdom (United Kingdom Clinical Research Collaboration, 2007), the need for recognition of, and a career pathway for research nurses in NZ (Walker, 2009b, 2010). Walker summarised her views in an article in the NZNO journal, Kai Tiaki (2009a), stating that research nurses deserve to be recognised for the "complexity and importance of the professional roles they perform and the specialised knowledge and skills they possess" (p. 26). She recommended that research nursing should be considered a speciality, and attention to career progression was required.

\section{Formulating the research question}

In formulating the research question, the literature was reviewed to see what studies had already been conducted and if they were relevant to the NZ setting. The term 'research coordinator' as a nurse was first used in the literature in 1970 (Kerman, 1970) and the term 'research nurse' in 1976 (Edwards, 1976). The volume of literature gradually increased throughout the 1980s and 1990s, with much of the earlier literature being experiential accounts about this emerging role (Ahern, Kruger, Gatcomb, Petit, \& Tamborlane, 1989; Chadwick, 1992; Fowler \& Stack, 2007; Mullin et al., 1984; Stephens-Lloyd, 2004; Waller, 2002). Issues which were evident in the literature included whether research nurses could be considered nurses as they were often regarded as data collectors by their colleagues (Kenkre \& Foxcroft, 2001) and whether research nursing could be seen as a specialty (RajaJones, 2002; Stephens-Lloyd, 2004). Expert opinion articles provided useful background information about research and the research nurse role, particularly about strategies to overcome challenges, such as the establishment of networks (Chatfield, 2008; Chester, Kennedy, Hynd, \& Matthews, 2007; Kenkre \& Foxcroft, 2001; Poston \& Buescher, 2010). While experiential accounts frequently echoed my own experiences, they did not provide evidence. Empirical studies began to emerge in the 2000s and are discussed in detail in Chapter 3.

While much was written about the role internationally, literature about research nurses in NZ has traditionally been combined with Australia in studies. Was the role different in NZ, or were the expectations and challenges faced the same? Much of the published literature is set in the United States of America (USA), which has a very different healthcare system to NZ. As will be shown in Chapter 3, there are five Australian publications about the research nurse which are set in ICU; however the NZ data component was small and not analysed separately. 
What better people to seek advice from when designing this study than the research nurses themselves? The ICU research nurses in NZ have become a cohesive group, with the establishment of annual meetings and an informal network in 2009. Before details of this research were finalised, I approached the NZ ICU Clinical Research Nurses Group at a meeting in August 2010, to gauge their level of interest and the feasibility of conducting this study. They were all enthusiastic about the idea and said they would like to participate. The possibility of including the PIs, who are the doctors they work closely with and their nurse managers (NMs) in the study was also broached with them. The nurses thought this was a good idea and that their PIs and NMs were likely to participate. Discussion about design and methods was also had with the group. Their main suggestions included keeping a diary as a way of recording their work for a period of time, and conducting a focus group. These suggestions are reviewed in Chapter 4 where the rationale for choosing a qualitative descriptive approach is discussed.

\section{Setting for research}

The setting for this study was six Level III ICUs in five NZ cities that employed at least one research nurse. Detailed information about NZ ICUs is included in Chapter 2 to provide context to the research and results. There are seven adult and one paediatric Level III ICUs in NZ. One of the adult ICUs was excluded because they did not have a permanent research nurse employed at the time this study was conducted. Although some Level II ICUs employ research nurses, the decision to include only Level III ICUs was pragmatic based on likely recruitment and volume of data. This decision was supported by a study about Australian and NZ ICU research coordinators, which showed that $84 \%$ of the respondents were from a Level III ICU (Rickard, Roberts, Foote, \& McGrail, 2006). Starship Paediatric ICU (PICU) was excluded because of the perceived different issues for research nurses working with paediatric patients compared to those in adult ICUs.

\section{Key terms}

\section{Research nurse}

The generally accepted definition for a research nurse is a nurse employed for the purpose of managing and coordinating clinical trials (Bell, 2009; Jeong, Kang, \& Kim, 2007; NZNO Critical Care Nurses' Section, 2011). Research nurses are referred to in various ways in the literature, including research coordinators, clinical research nurses, study coordinators, clinical trials coordinator and trial coordinators (Bell, 2009; Rickard et al., 2006). The 
terms which do not contain the word 'nurse' in the title refer to positions including, but not necessarily limited to nurses. Throughout this thesis, the original terms used in the literature or guiding documents are used. Unless otherwise stated, they refer to the research nurse role which is being investigated. For the purpose of this study, the generic term 'Research Nurse' and has been abbreviated to ResN.

It is important to distinguish between research nurses (ResN) and nurse researchers, as many articles that emerged from the literature were about nurse researchers. A nurse researcher is a nurse conducting their own research in the field of nursing (Gordon, 2008; Watmough, Flynn, Wright, \& Fry, 2010). Many ResNs do conduct their own studies, but they are mainly employed to conduct clinical trials (Hill \& MacArthur, 2006; Rickard et al., 2006). ResNs work in a variety of different clinical settings, including oncology, diabetes, cardiology, gastroenterology, paediatrics, and ICU (Rickard et al., 2006). In NZ, the work settings are varied but include universities, general practice, hospital and community locations in district health boards and private research institutions (Walker, 2009a, 2009b). There is no official data about how many ResNs work in NZ.

\section{Principal investigator}

In this thesis the principal investigator (PI) refers to an intensivist who is the investigator for at least one study in their ICU. In the ICU setting, the PI usually refers to the person who is responsible for the conduct of a clinical trial at that site and is usually an intensivist. This differs from the official definition for PI which is the person who has "primary responsibility for the design and conduct" (p. 103) of a study, and the person who conducts the study at a local site is usually called an 'investigator' (Ministry of Health [MOH], 2006). The PI for a study can be any health professional and as will be revealed in the findings, some of the research nurses who participated in this study were PIs for studies.

\section{Nurse managers and nurses}

The nurse in charge of ICU is known by different titles including Charge Nurse Manager, Nurse Unit Manager, and Nurse Manager. I use the term nurse manager (NM) when talking about this role. The registered nurses who are providing direct clinical care to ICU patients are referred to as 'bedside' nurses as this is what many participants referred to them as. Their official title is 'staff nurse' however 'bedside' nurse is used to differentiate between these nurses and research nurses. In some cases the research nurses also work as ICU 
bedside nurses, so the term research nurses is only used when referring to them in their research role.

\section{Other terms}

The terms 'role' and 'work' are used frequently throughout this thesis. Role is defined as "the function assumed or the part someone plays in a particular situation" (Oxford University Press, n.d.). 'Work' is about the tasks that are done. Other frequently used terms are provided in the glossary and further terms specific to the research are explained in Chapter 2, which gives an overview of the research process.

\section{Overview of the thesis}

This chapter has introduced the background to the research question and outlines the rest of the thesis. This thesis consists of seven chapters which collectively aim to describe the role of the research nurses working in NZ Level III ICUs. Chapter 2 gives some clinical context about the setting ICU research nurses work in. It is divided into three sections, of which the first gives the reader an overview of the healthcare setting and ICUs in NZ. The second section gives an insight into the process of research, and in particular, clinical trials. The third section brings the ICU and research specialities together to describe this challenging environment for conducting research. A review of the current literature about research nurses is presented in Chapter 3 and gaps are identified to frame the research question. Chapter 4 outlines the methodology for conducting the research study and describes how the research was conducted. The findings are presented in Chapters 5 and 6 . The focus of Chapter 5 is describing the participants and the ICU research departments while Chapter 6 presents the findings about the work of the ResN, in a thematic way. Chapter 7 discusses the findings, puts them in the context of the current literature and reflects on the study.

A key consideration in reporting the findings was managing confidentiality. Because the ICU research community is very small, and participants and ICUs are likely to be readily identifiable, discretion has been used as to when they are named, given code names, and when a general term, such as 'one research nurse said' is used. For the purpose of confidentiality, participants' identification numbers (e.g., ResN01) were randomly assigned. While it is acknowledged that readers of this thesis from ICUs in NZ may know and recognise who the participants are as a group, every endeavour has been made to protect individuals' views and opinions. 


\section{Chapter 2 Clinical context}

\section{Introduction}

This chapter contextualises the environment in which NZ ICU research nurses work. It provides a platform for the rest of the thesis by explaining the setting and organisations they work within and what is involved in conducting clinical research in NZ ICUs. This chapter has three distinct parts. It commences with a brief overview about the healthcare system and then introduces the reader to the ICU setting, definitions and the documents which guide ICU management. Part 2 introduces basic research concepts, including definitions of different types of research, phases of research and key guiding principles. The third part brings the specialty of ICU and research together and discusses considerations for conducting clinical research in ICU. Woven throughout is how the information provided links to the ResN.

\section{Part 1 New Zealand healthcare and ICU}

NZ publicly funded healthcare, including hospitals, is governed locally by 20 district health boards (DHBs) (Ministry of Health [MOH], 2010a). The DHBs were established under the NZ Public Health and Disability Act 2000 (NZ Government, 2000). In addition to publicly funded healthcare, there are many healthcare providers and hospitals which are privately funded through health insurance or patient self-payment.

\section{The Treaty of Waitangi}

The Treaty of Waitangi is a significant document in NZ history. It was signed in 1840 and aims to protect the rights and well-being of Maori, who are the indigenous people of NZ (Reid, 1999). This Treaty is essential when discussing research in NZ because Maori must be consulted prior to research occurring. Statistics show that Maori have a higher overall mortality than other ethnic groups and are overrepresented in many illnesses (MOH, 2010b) so considering Maori health interests is very important. Healthcare workers and providers have an obligation under the Treaty to ensure that Maori are not disadvantaged $(\mathrm{MOH}$, 2002). Furthermore, the Nursing Council of NZ (2011) state that the principles of the Treaty "require nursing to have a commitment to be responsive to Maori interests, and to ensure that these are protected" (p. 12). 


\section{Intensive care}

Intensive Care is defined by the Intensive Care Clinical Advisory Group (2005) as:

A designated ward of a hospital that is specially staffed and equipped to provide observation, care and treatment to patients with actual or potential life-threatening illnesses, injuries or complications, from which recovery is possible. The ICU provides special expertise and facilities for the support of vital functions and utilises the skills of medical, nursing and other staff trained and experienced in the management of these problems. (p. 8)

Guidelines for minimum standards for ICUs in NZ and Australia have been developed by the College of Intensive Care Medicine of Australia and New Zealand (CICM). CICM is the body which provides training, education and professional development for intensivists (CICM, 2012a). ICUs are categorised into three levels (Level I, II or III) according to the complexity of care they are able to provide and the number of patients they are capable of admitting annually (CICM, 2010b). A summary of the capabilities and differences between the three levels is provided in Table 1.

Level I ICUs are only required to provide immediate resuscitation and short term cardiorespiratory support for critically ill patients (CICM, 2010b). They need to be capable of providing mechanical ventilation and simple cardiovascular monitoring for several hours. This care could be provided for more than 24 hours but only if it involved failure of a single body system and a patient's care was discussed with an intensivist at a Level II or Level III ICU. Typically, critically ill patients are transferred to a Level II or Level III unit as soon as able.

A high standard of general intensive care under the supervision of an intensivist is expected in a Level II ICU. They can provide "complex multi-system life support, such as mechanical ventilation, renal replacement therapy and invasive cardiovascular monitoring for several days" (CICM, 2010b, p. 3).

Level III units are often referred to as tertiary referral ICUs, to which lower level units can refer their sickest patients. These units "should be capable of providing comprehensive critical care including complex multi-system life support for an indefinite period. Level III 
Table 1

\section{Requirements for the 3 levels of Intensive Care Unit in NZ*}

\begin{tabular}{|c|c|c|c|}
\hline & Level I & Level II & Level III \\
\hline Overview & $\begin{array}{l}\text { Immediate resuscitation \& short term } \\
\text { cardio-respiratory support }\end{array}$ & High standard of general intensive care & Tertiary Referral Unit \\
\hline $\begin{array}{l}\text { Type of care } \\
\text { provided }\end{array}$ & $\begin{array}{l}\text { Simple invasive cardiovascular monitoring } \\
\& \text { mechanical ventilation for several hours }\end{array}$ & $\begin{array}{l}\text { Invasive cardiovascular monitoring, renal } \\
\text { replacement therapy, mechanical } \\
\text { ventilation for several days }\end{array}$ & $\begin{array}{l}\text { Complex multi-system life support } \\
\text { indefinitely }\end{array}$ \\
\hline $\begin{array}{l}\text { Work } \\
\text { practice/caseload }\end{array}$ & $\begin{array}{l}\text { Sufficient ICU beds \& admissions to } \\
\text { maintain clinical skills of medical \& } \\
\text { nursing staff }\end{array}$ & $\begin{array}{l}\text { Minimum } 4 \text { staffed \& equipped beds } \\
>200 \text { mechanically ventilated patients } \\
\text { each year }\end{array}$ & $\begin{array}{l}\text { Minimum } 6 \text { staffed \& equipped beds } \\
>300 \text { mechanically ventilated patients } \\
\text { each year }\end{array}$ \\
\hline \multirow{3}{*}{ Medical Staff } & $\begin{array}{l}\text { Medical director who is experienced in } \\
\text { Intensive Care Medicine }\end{array}$ & $\begin{array}{l}\text { Medical director (Fellow of College of } \\
\text { Intensive Care Medicine) }\end{array}$ & $\begin{array}{l}\text { Medical director (Fellow of College of } \\
\text { Intensive Care Medicine) }\end{array}$ \\
\hline & $\begin{array}{l}\text { Consultant support from a specialist with } \\
\text { ICU experience }\end{array}$ & $\begin{array}{l}\text { One other specialist who is a Fellow of } \\
\text { College of Intensive Care Medicine }\end{array}$ & $\begin{array}{l}\text { Sufficient specialists so that an ICU } \\
\text { specialist is always available }\end{array}$ \\
\hline & $\begin{array}{l}\text { At least } 1 \text { medical practitioner exclusively } \\
\text { rostered at all times }\end{array}$ & $\begin{array}{l}\text { At least } 1 \text { medical practitioner exclusively } \\
\text { rostered at all times }\end{array}$ & $\begin{array}{l}\text { At least } 1 \text { medical practitioner exclusively } \\
\text { rostered at all times }\end{array}$ \\
\hline \multirow{6}{*}{ Nursing Staff } & $\begin{array}{l}\text { Nurse in charge must have post- } \\
\text { registration qualification in ICU or the } \\
\text { specialty of the unit }\end{array}$ & $\begin{array}{l}\text { Nurse in charge must have post- } \\
\text { registration qualification in ICU or the } \\
\text { specialty of the unit }\end{array}$ & $\begin{array}{l}\text { Nurse in charge must have post- } \\
\text { registration ICU qualification }\end{array}$ \\
\hline & $\begin{array}{l}\text { Majority of nursing staff have post- } \\
\text { registration qualification in ICU or the } \\
\text { specialty of the unit }\end{array}$ & $\begin{array}{l}\text { Majority of nursing staff have post- } \\
\text { registration qualification in ICU or the } \\
\text { specialty of the unit }\end{array}$ & $\begin{array}{l}\text { Majority of nursing staff have post- } \\
\text { registration ICU qualification }\end{array}$ \\
\hline & & Access to a nurse educator & At least one nurse educator \\
\hline & $\begin{array}{l}\text { All nurses responsible for patient care are } \\
\text { registered nurses }\end{array}$ & $\begin{array}{l}\text { All nurses responsible for patient care are } \\
\text { registered nurses }\end{array}$ & $\begin{array}{l}\text { All nurses responsible for patient care are } \\
\text { registered nurses }\end{array}$ \\
\hline & 1:1 nursing for critically ill patients & 1:1 nursing for critically ill patients & $\begin{array}{l}\text { 1:1 nursing for ventilated or critically ill } \\
\text { patients }\end{array}$ \\
\hline & $\begin{array}{l}\text { Minimum of } 2 \text { registered nurses in unit at } \\
\text { all times, when there is a patient admitted } \\
\text { to the unit }\end{array}$ & & $\begin{array}{l}\text { Nursing staff available for }>1: 1 \text { ratio in } \\
\text { very complex patients }\end{array}$ \\
\hline
\end{tabular}




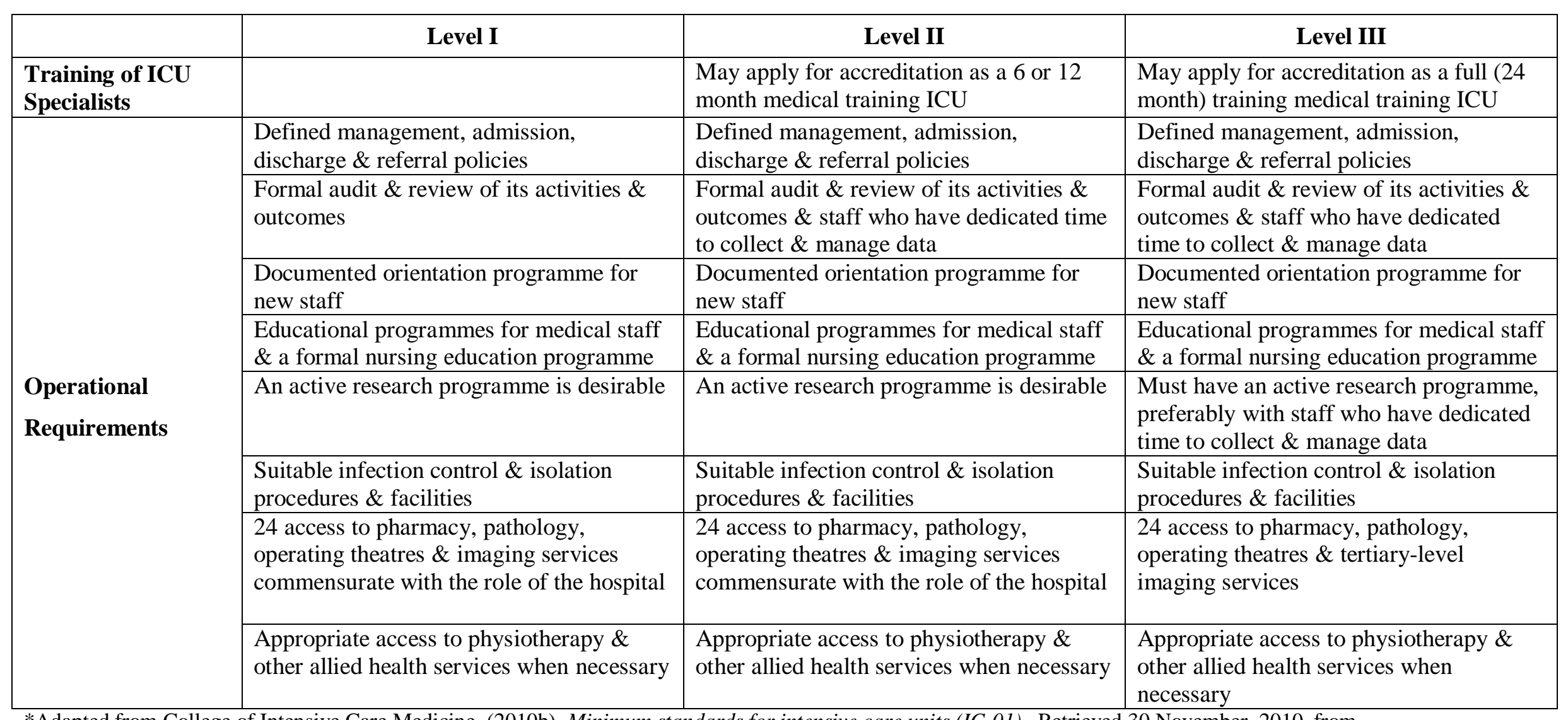

*Adapted from College of Intensive Care Medicine. (2010b). Minimum standards for intensive care units (IC-01). Retrieved 30 November, 2010, from

http://www.cicm.org.au/cmsfiles/IC-1\%20Minimum\%20Standards\%20for\%20Intensive\%20Care\%20Units.pdf 
units should have a demonstrated commitment to academic education and research" (CICM, 2010b, p. 1).

Level III ICUs can apply for accreditation to provide medical or registrar training in intensive care medicine for six, 12 or 24 months and Level II ICUs for six or 12 months as per the requirements outlined in the CICM IC-3 guideline (2010a). Pertinent to the ResN role, training accredited ICUs are required to have:

Access to an appropriate level of funding for research coordinators to support the active research program. As a minimum, funding to support the full time research coordinator position for a Level III Unit (IC-1), and an appropriate part-time position in smaller units. (CICM, 2010a, p. 4, S. 3.5)

\section{New Zealand ICUs}

The Acute Respiratory Unit at Auckland Hospital, established in 1962 was the first dedicated ICU in NZ (Trubuhovich \& Judson, 2001). There are currently 22 adult ICUs in NZ public hospitals, and a further three in private hospitals (Table 2). Many ICUs also admit children, and Starship Hospital has a dedicated paediatric ICU. Neonatal ICUs are not included in this table as they are regarded as a separate specialty. Auckland City Hospital Department of Critical Care, Christchurch Hospital, Middlemore Hospital and Wellington Hospital are accredited as unrestricted 24 month training ICUs (CICM, 2012b). Auckland City Hospital Cardiovascular ICU, Hawke's Bay Hospital, and Waikato Hospital are approved for 12 month advanced training, and North Shore Hospital for six months.

There were 18,919 intensive care and high dependency admissions to NZ ICUs in 2008 (ANZICS Centre for Outcome and Resource Evaluation Database, [ANZICS CORE Database], 2011). Of the 10,473 admissions to Level III ICUs in this period, $42 \%$ were classified as emergency admissions and the remainder were following elective surgery. The mortality rate varies depending on reasons for admissions and admission diagnoses; however the overall mortality rate in 2008 for Level III ICUs was $7 \%$.

Due to the severity of their illness, ICU patients are vulnerable in clinical and research terms. They are often unconscious or sedated, and therefore unable to speak for themselves. This is referred to as 'incompetence' and is the (usually temporary) inability of a patient to "understand information presented, to appreciate the consequence of acting (or not acting) on 
that information and to make a choice" for themselves (MOH, 2006, p. 101). In 2008, approximately $52 \%$ of patients admitted to Level III ICUs were mechanically ventilated and therefore would have been sedated at some point during their admission (ANZICS CORE Database, 2011) meaning they were unable to communicate verbally.

Table 2 Summary of New Zealand Intensive Care Units by level*

\begin{tabular}{|c|c|c|}
\hline Level I & Level II & Level III \\
\hline Gisborne Hospital & Hawke's Bay Hospital & $\begin{array}{l}\text { Auckland City Hospital } \\
\text { Cardiovascular ICU }\end{array}$ \\
\hline Grey Hospital & $\begin{array}{l}\text { Mercy Hospital \& Health } \\
\text { Services** }\end{array}$ & $\begin{array}{l}\text { Auckland City Hospital } \\
\text { Department of Critical Care } \\
\text { Medicine }\end{array}$ \\
\hline Hutt Hospital & North Shore Hospital & Christchurch Hospital \\
\hline $\begin{array}{l}\text { Southern Cross } \\
\text { (Hamilton)** }\end{array}$ & Palmerston North Hospital & Dunedin Hospital \\
\hline Taranaki Base Hospital & Rotorua Hospital & Health Waikato \\
\hline Wairau Hospital & Southland Hospital & Middlemore Hospital \\
\hline Wakefield Hospital $* *$ & Tauranga Hospital & Starship Children's Hospital \\
\hline Whakatane Hospital & Timaru Hospital & Wellington Hospital \\
\hline & Wanganui Hospital & \\
\hline & Whangarei Hospital & \\
\hline
\end{tabular}

Ventilated and critically ill ICU patients are nursed at a 1:1 ratio (one nurse for each patient). Patients who are less sick and considered 'high dependency' may be nursed at a 1:2 ratio (Morley, 2005). Because of these ratios, the bedside nurse constantly monitors and assesses the patient's condition, makes clinical decisions and provides care for the patient, in 
collaboration with medical and allied health staff. In NZ, ICU patients are cared for by a registered nurse (CICM, 2010b).

\section{Part 2 Overview of research principles}

Research is a systematic and methodical process which aims to create new knowledge or validate existing knowledge (European Medicines Agency [EMA], 2002; Gillis \& Jackson, 2002; Rischbieth \& Blythe, 2005). There are many different types of research, however because most research conducted in NZ ICUs is observational or clinical trial design, these two designs are described in more detail to give an indication of the expectations of the Res $\mathrm{N}$ in such studies.

Observational studies refer to studies which observe current practice, and no intervention takes place (National Ethics Advisory Committee [NEAC], 2006). These studies involve only data which can be collected prospectively or retrospectively. The data are then analysed and can be used for quality control, benchmarking current practice and to "add to generalisable knowledge about a health issue or disability" (NEAC, 2006, p. 3). Low risk observational studies are eligible for review by an expedited ethical approval process.

A clinical trial is an investigation in human subjects, intended to discover or verify the "safety, efficacy or optimum dosage schedule of an investigational product or intervention" (Rischbieth $\&$ Blythe, 2005, p. 60). It is usually pre-planned and participants are selected according to "predetermined eligibility criteria" (Rischbieth \& Blythe, p. 60) and assessed according to predefined endpoints.

There are four phases of clinical trials (Coulson \& Phelan, 2000; Fowler \& Stack, 2007; Murray, 2011). The focus of Phase I studies is to collect safety data and the research drug or therapy is used for the first time in healthy humans. Phase II studies assess whether the agent has an effect on the disease (efficacy) in a particular group of patients. The purpose of a Phase II study may be to see if a Phase III study is feasible and warranted. Because Phase II studies have a small patient population and patient outcomes may not show an effect, surrogate endpoints such as biomarkers or hospital length of stay may be used. Phase III studies gather additional safety and efficacy data and aim to show whether the new agent is statistically and clinically better than best current practice. The follow-up tends to be longer, and have a patient 
based outcome such as mortality or quality of life. Phase IV studies are long term and done when the therapy is licensed and in general use. Most clinical trials in ICU are Phase II and III.

\section{Good clinical practice}

Fundamental to all clinical trials is the concept of good clinical practice (GCP). "GCP is an international ethical and scientific quality standard for designing, conducting, recording and reporting trials that involve the participation of human subjects" (EMA, 2002, p. 5). The purpose of GCP is to ensure that patients' rights are upheld and research studies are conducted in an ethical way. The origins of GCP are in the Declaration of Helsinki which state that the individual patient's rights take precedence over the interests of a study (World Medical Association, 1964). The risks of a trial must be carefully weighed against the likely benefits. Underpinning GCP is patient consent and the concept that the patient has the right to refuse to participate in a research trial and is still entitled to receive the best available medical care. Responsibilities of those involved in research are outlined in the Guideline for Good Clinical Practice (EMA, 2002). Since January 2011, all clinical trials conducted in NZ have been expected to adhere to GCP guidelines ( $\mathrm{MOH}, 2011)$.

The role of the trial 'monitor' is to ensure that a study complies with GCP. This is done by checking that enrolled patients met eligibility requirements, consents are filled in correctly and that pre-specified data points match source data (EMA, 2002). Source data are defined as the information found in source documents which are the "original records or certified copies of original records of clinical findings" (p. 10) such as hospital records, laboratory results, radiology results and vital observations (EMA, 2002). The sponsor is the research organisation "who take responsibility for the initiation, management and financing of a clinical trial" (EMA, 2002, p. 10).

\section{Protocol}

The process of conducting a clinical trial begins with recognising an issue or problem that needs to be investigated. The protocol is the document which outlines all information about a trial. It provides the statistical and clinical background, rationale based on previous studies and literature to conduct the trial, specifies treatments to be performed, the data to collect and safety information, including the course of action if a patient is put at risk, (EMA, 2002). The protocol is developed and refined by the investigator and/or management committee, which is a group of interested and suitably qualified individuals. The protocol is submitted for approval 
by an ethics committee, along with any written study material, including information sheets, consent forms and advertising information. Adherence to the protocol ensures that procedures are carried out consistently and safely by multiple participating sites.

Protocol violations (or deviations) are events which are not in accordance with the study protocol. They may or may not be harmful to the patient, but may put the results of the study in jeopardy, by diluting the statistical effect. An example of a protocol violation would be if a study was investigating the efficacy of two different resuscitation fluids and a patient was given the incorrect fluid, not the one they were randomised to receive. This type of violation requires reporting to the sponsor. Serious adverse events (SAEs), on the other hand, are events that may have put the patient at risk, and may or may not be related to the study treatment. EMA (2002, p. 10) defines SAEs as:

Any untoward medical occurrence that at any dose:

- $\quad$ results in death,

- $\quad$ is life-threatening,

- $\quad$ requires inpatient hospitalization [sic] or prolongation of existing hospitalization, results in persistent or significant disability/incapacity, or

- $\quad$ is a congenital anomaly/birth defect.

A data monitoring committee (DMC) is responsible for the safe conduct of the study and unlike the management committee they can review unblinded data while the study is in progress. A DMC consists of "a group of independent experts external to a study assessing the progress, safety data and, if needed critical efficacy endpoints of a clinical study" (EMA, 2005, p. 3). If the trial does not have a DMC, the PI must report any SAE to the ethics committee as soon as able (New Zealand Health and Disabilities Ethics Committees [HDEC], 2010). If a trial is overseen by a DMC, SAEs are reported to the ethics committee for NZ patients only if the SAE is unexpected, not defined by study end-points, and if the study code must be unblinded (HDEC, 2010).

\section{Conducting research and clinical trials in New Zealand}

In addition to GCP, researchers must also adhere to the ethical requirements for NZ. Changes to the NZ Health and Disability Ethics Committees (HDEC) became effective on 13 June 2012 while this thesis was in the final stages of being written up (HDEC, 2012). Because data 
collected about the ResN's role in ethics were collected in 2011, the ethical review requirements in effect at the time of the interviews are discussed below, not the new guidelines.

\section{$N Z$ ethical review process}

Ethical review is the approval of research by an external body to determine whether the research being proposed has an acceptable risk to the patient compared to the likely benefit to society. In NZ, six regional and one multi-regional ethics committees (MREC) were established in 2004, under Section 11 of the NZ Health and Disability Act 2000 (NZ Government, 2000) and operate under the MREC Committee Terms of Reference (HDEC, n.d.). The primary purpose of ethical review is to ensure "that the rights and interests of participants in research and innovative practice, and consumers of health and disability services" are protected $(\mathrm{MOH}, 2006$, p. 2). MREC review allows for a single ethics application, submitted by the 'lead site', when a study is being conducted at more than one site in NZ.

If multiple ICUs are participating in a study, it is my experience that the research staff at one of the ICUs volunteers to be the lead site. Staff at the lead site complete the full ethics application and create generic consent forms and information sheets for the NZ setting. These forms and sheets are either adapted from a template sent by the sponsor or developed independently. The documents from all other sites are collated by the lead site and submitted to MREC together. The approvals necessary from each site are evidence of consultation with Maori, signed locality assessment, and a signed declaration by each PI (called a 'Part 4'). Evidence of consultation with, and support from Maori is completed according to the local hospital process. The locality assessment is a declaration that the proposed locality is appropriate and has sufficient resources, including personnel, for the trial to take place (MOH, 2006). The Part 4 declaration is a statement signed by the PI and senior manager stating that the PI understands the protocol and ethical considerations have been made.

If a drug is a 'new medicine', approval must first be given by the Standing Committee on Therapeutic Trials (SCOTT) under Section 30 of the Medicines Act 1981 (MOH, 2011). The purpose of SCOTT approval is to assess the scientific and clinical validity and risks of the proposed trial. Additionally, SCOTT reviews the merit of clinical trials being submitted for Health Research Council funding (Health Research Council of New Zealand, 1993). 


\section{Hospital research departments}

In planning this study, I presumed that most hospitals or DHBs had a research department or policy to ensure that the DHB is protected from risk (including financial) when research is conducted within the hospital. The hospital I work at has a register of research being conducted within the hospital. The budget and clinical trials agreement are assessed prior to the commencement of any research.

\section{Study funding}

Generally, a 'per patient' payment for each patient enrolled into a study is paid by the sponsor to the site. The 'per patient' payment aims to recompense the costs of the study, including ResN time, consumables, therapeutic substances, and laboratory, pharmacy and radiology costs. Study funding is an important consideration for this thesis because in NZ ICUs, funding received from studies contributes to the cost of ResNs' salaries. Specific details about this are discussed in Chapter 5.

\section{Commercial studies}

Commercially sponsored studies are those where a clinical trial is "conducted principally for the benefit of the manufacturer or distributor of the medicine or item being trialled" (HDEC, 2012, p. 52). Commercial studies tend to be drug trials which may be experimental in nature or assessing a new use, different doses or side effects for an existing drug. Sites are paid by pharmaceutical or other healthcare companies.

\section{Investigator-initiated studies}

Investigators initiating their own studies can apply for grants from research organisations such as the Health Research Council (HRC), private trust funds and special interest groups or collaboration may be sought from commercial companies, to provide an unconditional grant. Anecdotally, investigator-initiated, grant-funded studies tend to be less well funded than commercial studies, and sometimes the 'per-patient' payment may not cover the costs incurred. Observational studies are usually investigator-initiated and may attract little or no funding.

\section{Part 3 Bringing two specialties together - research in the ICU setting}

Research has been a part of ICU for almost as long as ICU has existed in NZ. While it is difficult to say exactly when research started in ICUs, when 'New Zealand', 'Intensive Care' and 'Critical Care' are searched in Medical Literature On-Line via ProQuest (Medline) and Cumulative Index to Nursing and Allied Health Literature (CINAHL), the earliest NZ 
publication was an article published in 1967 titled 'The Intensive Care Unit in Tauranga' (Fergus \& Watts, 1967). The intensivists profiled in 'Intensive Care in New Zealand - a History of NZ Region of ANZICS' (Trubuhovich \& Judson, 2001) talked about conducting research at their ICUs throughout the 1980s and 1990s. NZ ICUs have also been involved in many recent multicentre international studies (Cooper et al., 2011; NICE Study Investigators, 2009; The ANZIC Influenza Investigators, 2009).

\section{What is known about the research nurse role?}

The ResN role is relatively new and the first nurse employed specifically as a ResN in a NZ ICU was in 1997 in Auckland Hospital Department of Critical Care Medicine (L. Newby, personal communication, June 15, 2010). The exact number of ResNs working in NZ ICUs is unknown, however all but one Level III ICU employed at least one ResN prior to this study.

\section{Trial selection}

The process of studies being selected by an ICU relies on a belief of 'equipoise' within the ICU. Equipoise is where there is genuine uncertainty about a treatment (Murray, 2011). The protocol will be assessed by ICU personnel to establish the feasibility of conducting the study in their ICU. Who is involved in this decision in the participating ICUs is revealed in Chapter 6. Determining feasibility involves estimating the number of patients a site could recruit and can also include consideration of the costs which will be incurred. Usually one of the intensivists will become the PI for a trial, or for observational studies, this may be a ResN or other doctor such as a registrar. Sites are selected by the sponsor based on the ICU's ability to recruit patients into a study and their research experience.

\section{Considerations when conducting research in ICU}

ICU patients are a vulnerable cohort and there are many ethical and clinical considerations when conducting research in ICU. With approximately half of all admissions being classified as emergency (or unexpected) admissions, ICU can be a frightening experience for the patient and their family. ICU patients can be extremely sick and many will die of their illness either in ICU, or soon after (Hicks \& Mackle, 2010).

\section{Consent}

Consent is the process by which a patient decides whether to participate in a trial or not. This process involves explaining the patient's condition, the specifics of the study treatment and potential risks and benefits of the study. The information must be given to the patient in a way 
they can understand. This is usually in the form of a 'plain language' information sheet and the option of an impartial interpreter if required. Fundamental to upholding patients' rights, the patient must consent without "manipulation, coercion, inducement or any other undue influence" (MOH, 2006, p. 7).

As ICU patients are frequently unable to consent to participate in clinical trials for themselves, special provision is provided in Research involving unconscious participants (Appendix 3) of the Operational Standards for Ethics Committees (MOH, 2006). Unconscious patients can be enrolled into studies either using a 'delayed consent' option or after consultation with their family (assent) (Table 3). 'Assent' is a term used by some ICU researchers and refers to obtaining permission from the patient's family to enrol a patient into a trial. In NZ law, there is no allowance for consenting by proxy (unless the patient has a legal representative), so when research is discussed with family, they are asked if they think the patient would want to participate in a study.

Table 3 Types of consent used in the ICU setting

\begin{tabular}{|l|l|l|}
\hline & \multicolumn{1}{|c|}{ Consent (patient) } & \multicolumn{1}{c|}{ Assent (family) } \\
\hline Prior & $\begin{array}{l}\text { Prior consent from the patient is the } \\
\text { usual practice when conducting } \\
\text { research. This is obtained from the } \\
\text { patient before any treatment begins. }\end{array}$ & $\begin{array}{l}\text { Assent is where the family agrees to } \\
\text { their family member participating in a } \\
\text { trial prior to study treatment beginning. }\end{array}$ \\
\hline Delayed & $\begin{array}{l}\text { If no family is immediately available, } \\
\text { the patient may be enrolled into an } \\
\text { approved study without consent. Once } \\
\text { the patient is able to consent, they } \\
\text { consent to continuing with the study. } \\
\text { This may be following assent from the } \\
\text { family or from the patient alone. }\end{array}$ & $\begin{array}{l}\text { If no family is immediately available, } \\
\text { the patient may be enrolled into an } \\
\text { approved study and assent sought from } \\
\text { the family after the study treatment has } \\
\text { begun. }\end{array}$ \\
\hline
\end{tabular}

Delayed consent allows the patient to be enrolled into some trials without prior consent or consultation with the family. The use of delayed consent is applied for on an individual trial basis as part of the ethics application and is used only when the family is not immediately available. This request is made for trials where delaying enrolment into the trial until the family had been spoken to, would mean that many patients were not able to be enrolled. An 
example of this is for a study investigating fluid resuscitation, where treatment must be given immediately. Assent to continue with study treatment is sought from the family, until the patient is competent to decide for themselves.

Whichever option is used, consent to continue with the study is sought from the patient when they are competent and they still have the right to decline any further study treatment or follow-up, without affecting their medical treatment. If the patient does not recover enough to consent, or dies, the ethics committee can give permission for the trial data to be used.

The decision about who can obtain consent from a patient is trial-specific and would generally be decided at the beginning of each study, and documented in a 'delegation of duty log', which is authorised by the PI. Depending on the study, they may perform all of the tasks themselves, or delegate them to someone else.

\section{Other considerations}

The timeframe for putting a patient into an ICU clinical trial is often very short. While all clinical trials are scientifically justified and ethically approved, the full effect of study treatment is unknown; therefore there is always a risk. All potential risks and benefits of a study are explained to the family (or patient) and they have the opportunity to ask questions. Many ICU studies are 'blinded', which means none of the staff know if the patient will receive the treatment or if they are in the control group. Part of the consent process includes explaining to families that clinical trials are only conducted when it is not known if one treatment is more beneficial than another in this group of patients. The family are asked to consider the risks and benefits and make the decision about allowing the patient to participate. This can be a burdensome decision to make in an already stressful situation as their family member is critically ill and they are in an unfamiliar environment, perhaps without their usual support networks.

\section{Supporting organisations}

\section{Australia New Zealand Intensive Care Society Clinical Trials Group}

The Australia New Zealand Intensive Care Society (ANZICS) is a professional body for intensivists (ANZICS, 2010) of which the ANZICS Clinical Trials Group (CTG) is a subcommittee (CTG, 2011a). Established in 1994, the CTG is a group of intensivists and researchers, whose members are committed to collaborating to produce high quality research 
(CTG, 2010a). The CTG is funded by ICUs paying an annual fee and eight NZ ICUs are members (CTG, 2011a). Three scientific meetings are run by the CTG each year and are open to all staff including PIs, other doctors and ResNs. The largest meeting runs over three days in March and is held in Australia. At all of the meetings, there are presentations about studies at all stages of development, updates about current studies and results from studies which have been completed. When new studies are presented, there is an open forum for feedback about the design, potential problems and feasibility, which anyone present can contribute to.

Although the CTG does not conduct studies itself, investigators can seek 'CTG-endorsement' for their studies. The process of CTG-endorsement involves the investigator presenting the study at a CTG meeting and submitting documentation about the study, which is then assessed by the CTG executive committee. Relevant to the ResN role, a condition of CTG-endorsement it that "the study management committee must include at least one individual employed as an ICU Research Coordinator [sic] for the duration of the study" (CTG, 2011c, p. 8). Many multi-centre studies have been endorsed by the CTG, and several publications in international medical journals have resulted (Cooper et al., 2011; NICE Study Investigators, 2009; The ANZIC Influenza Investigators, 2009; The Blood Observational Study Investigators on behalf of the ANZICS-Clinical Trials Group, 2010). An example of an ongoing CTG-endorsed observational study is the 'point prevalent' programme which ICUs across Australia and NZ can participate in once or twice a year. As well as reporting a 'snapshot' of patients who are in the ICUs on the specified days, point prevalent days are also used to assess current practice about pre-defined topics, how many patients are enrolled in research trials and feasibility of future research projects. These point prevalent studies are also used to see whether research findings have been translated into clinical practice (Finfer et al., 2010). Researchers can apply to have questions included in a point-prevalent study (CTG, 2010b).

It is unknown if enrolling patients concurrently into more than one study, known as coenrolment, is common practice in other clinical areas. For CTG-endorsed studies, coenrolment is encouraged and outlined in the CTG Co-enrolment Policy (CTG, 2011b) to ensure that trials are completed as quickly as possible "allowing results of potentially beneficial interventions to be available earlier" (p. 1). The management committee for each study decides which studies it is safe to co-enrol in, without compromising patient safety or potentially affecting the study outcomes. There is also a CTG Competing Studies Policy which prioritises 
studies which are CTG-endorsed and funded by large funding bodies such as the HRC in NZ or National Health and Medical Research Council (NHMRC) in Australia, to ensure trials are completed on time (CTG, 2008).

\section{Intensive Care Research Coordinators Interest Group (IRCIG)}

The Intensive Care Research Coordinators Interest Group (IRCIG) is a collaborative network for research coordinators and is a sub-group of the CTG (IRCIG, 2011, 2012). IRCIG was formally established in 2000 and was originally called the Intensive Care Research Nurses Interest Group. The name was changed in 2001 to incorporate people doing the research role, who were not nurses (B.L. Roberts, personal communication, 16 ${ }^{\text {th }}$ April, 2012). The title Research Coordinator (RC) is used throughout Australia, and includes, but does not refer exclusively to nurses (Rickard et al., 2006).

IRCIG aims to provide support for RCs, promote the role of RCs and to enable communication between them. A significant aim is to ensure that CTG-endorsed studies receive input from RCs through representation on the committees that develop the studies (IRCIG, 2011). IRCIG coordinates a regional mentoring programme for RCs throughout Australia and NZ, with one representative in NZ. IRCIG has a one-day workshop the day before the main CTG meeting which has guest speakers and offers educational and professional development talks. ResNs are encouraged to present their own research work. It also provides an opportunity for ResNs to network with others in the same position. It was at one of these annual workshops that the idea of starting a NZ based network for ICU ResNs began. There are few NZ based ICU ResNs, compared to Australia and it was recognised that NZ needed a collective voice.

\section{New Zealand Critical Care Clinical Research Nurse Group}

In August 2009, the NZ ICU Research Nurses Group was started with a meeting in Wellington. This first meeting had two invited speakers, speaking about ethics and the role of the ResN. Each of the nine ICU ResNs present spoke about the projects they were currently involved in. Information about the member units, including the studies they were participating in, contact details and ResN full-time equivalence (FTE), was collated at this meeting, and updated with the second meeting at Middlemore ICU in 2010. In 2010, the ICU ResNs became the first subgroup of the Critical Care Section of the NZ Nurses Organisation, and became known as the NZ Critical Care Clinical Research Nurse Group. A position statement about the role of 
the research nurse in ICU was released in January, 2011 (NZNO Critical Care Nurses' Section, 2011).

\section{Summary}

This chapter has outlined healthcare and ICU in NZ, research concepts and given some background about the way research is incorporated into ICU in NZ. Challenges of conducting research in the ICU setting have been outlined, as well as organisations which support research and ResNs working in ICU.

By providing this background to conducting research in NZ ICUs, a base has been provided, on which the international literature, choice of methodology and ultimately the findings of this theses can be built. The next chapter builds on contextualising the research by analysing the international literature about the role of the ResN. 


\section{Chapter 3 Literature review}

\section{Introduction}

A review of the literature was conducted to see what was already known about the role of the ResN internationally, to assess whether it was reflective of the NZ setting and to identify gaps in current knowledge. This chapter begins with an outline of the search strategy and inclusion criteria. An overview of the literature and its quality is then provided, following which the findings and an analysis of relevant international literature are presented. The themes that emerged and were considered significant include the development of the role, the 'work' of ResNs, their role preparedness and job satisfaction. The gaps in the literature are identified in the conclusion, paving the way for the topic of this thesis.

\section{Search strategy}

A literature review was conducted at the outset of this study in 2010, and updated again in 2012. Four significant studies were published between the initial and final review. The literature review was conducted using CINAHL, Medline and PubMed databases, and Google Scholar search engine. Various combinations of the search terms "research nurse", "research coordinator", "study coordinator", "clinical trials coordinator", "clinical research nurse" and "trials coordinator" were used as keywords. These results were combined with the term 'role' and limited to articles in the English language, published from 2000 - 2012. Publications these articles cited were also reviewed, as were significant articles cited in other literature (Figure 1). This resulted in a total of 21 articles, which were reviewed in depth. These articles were based on 16 individual studies; three of the studies involved more than one publication (Mueller, 2001; Mueller \& Mamo, 2000, 2002; Rickard et al., 2006; Rickard, Roberts, Foote, \& McGrail, 2007; Roberts, Eastwood, Raunow, Howe, \& Rickard, 2011a, 2011b; Roberts, Rickard, Foote, \& McGrail, 2006). A review article (Becze, 2010) which summarised the article by Nagel, Gender and Bonner (2010) was not analysed separately as it contained no new information.

Studies were only included if they had an empirical study component. Articles were excluded if they were about nurse researchers or had no information about the role of the ResN. The hierarchy of evidence and research in Moule and Goodman (2009) ranks 'expert opinion' and 'experiential accounts' in the lowest two positions. While experiential accounts give a valuable insight into the 'day-to-day' work that ResNs do and are often cited in publications, 
Figure 1 Search strategy

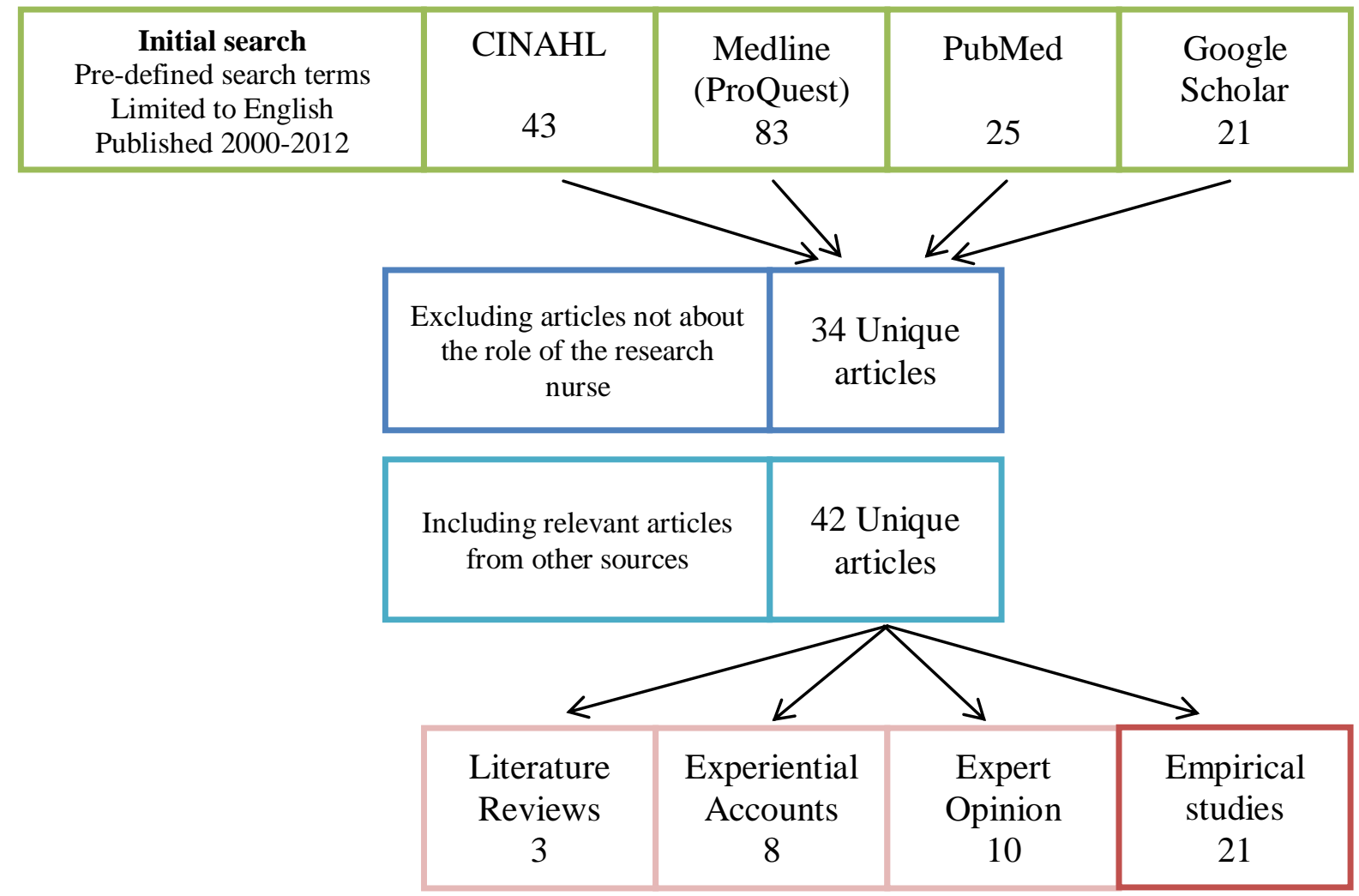

they have been excluded from this literature review because of this low ranking. Likewise, although there was useful background information in expert opinion articles they were not analysed in isolation. Three articles were excluded from the final analysis because they were literature reviews (Bell, 2009; Bird \& Kirshbaum, 2005; Raja-Jones, 2002).

\section{Overview of literature}

The majority of studies about the role of the ResN are from the United States of America (USA) $(\mathrm{n}=9)$, one of which included participants from Canada. There are also studies from Australia $(n=6)$, Italy $(n=1)$, South Korea $(n=1)$, Spain $(n=1)$ and United Kingdom $(U K)$ $(n=3)$. Five of the Australian publications involved NZ respondents. The publications most relevant to this thesis are five articles, based on two separate surveys, which are set in Australian and NZ ICUs (Rickard et al., 2006, 2007; Roberts et al., 2011a, 2011b; Roberts et al., 2006). In some sections of this review, these studies have been analysed separately from the rest of the literature. Studies which relate to clinical areas other than ICU have been included in the overall analysis of the literature, to position the role in a more general context. 
In all except one study (Catania et al., 2012), the ResNs were predominantly female (85 $100 \%$ ), with an age range of 21 - 65 years. Age was recorded using either a range or the mean. Where a mean age was provided, this ranged from 30.9 - 45.8 years. Where the information was collected, ResNs were well educated and experienced nurses, although it is not clear whether this was a prerequisite for the role or if there was an expectation (either formally or informally) to attain these qualifications once in the role.

Eight studies which included non-nurse research coordinators in the cohort have been reviewed because they contribute to what is known about the role. The non-nurse study coordinators came from a variety of backgrounds including general sciences, public health, genetic, physiotherapy, and social work (Anderson, 2008; Davis, Hull, Grady, Wilford, \& Henderson, 2002; Loh, Butow, Brown, \& Boyle, 2002; Rickard et al., 2006, 2007; Rico-Villademoros et al., 2004; Roberts et al., 2006). Table 4 summarises the method, participants, findings and relevance to this study. In this table, unless otherwise stated, the participants are nurses. Methods used for data collection included survey $(n=13)$, focus groups $(n=3)$, one-to-one interviews $(n=3)$ or a combination $(n=1)$. The method was not articulated for one study (Gordon, 2008). All but one of the studies examined information from the viewpoint of the ResN only. The remaining study surveyed ResNs and research doctors (investigators) about the role of the research nurse (Jeong et al., 2007).

Rickard et al. (2006) reported that out of the 49 respondents in a study of intensive care (ICU) research coordinators (RCs), there were 21 different job titles, and $31 \%$ had no job description. Few studies surveyed the structure of research departments, or analysed the reporting lines for ResNs. The RCs reported most commonly to an intensivist (53\%), nurse manager (16\%) or both (18\%). Hill and MacArthur's UK study (2006) reported 17\% of ResNs had a clinical nurse line manager. These authors reported that a significant number of respondents $(65 \%)$ were on fixed-term contracts and although $72 \%$ of participants had a job description, only $50 \%$ considered it accurately reflected their duties. Concerns were raised about short-term contracts and having to recruit patients in order to pay for their wages by Hill and Macarthur. This was echoed by Rickard et al. who found that $37 \%$ of respondents "would remain in the role until the funding runs out" (p. 236), although this was not an issue for other respondents. 
Table 4 Summary of relevant literature about the role of research nurses

\begin{tabular}{|c|c|c|c|c|c|}
\hline Reference & Country & Method & $\begin{array}{c}\text { Participants and } \\
\text { setting }\end{array}$ & Research aim & Findings in relation to this study \\
\hline $\begin{array}{l}\text { Anderson } \\
(2008)\end{array}$ & USA & Survey & $\begin{array}{l}\text { Study Coordinators } \\
\text { (nurses, } \mathrm{n}=35 ; \\
\text { non-nurses, } \mathrm{n}=55 \text { ) } \\
\text { Gene therapy }\end{array}$ & $\begin{array}{l}\text { Describe characteristics of } \\
\text { sample study coordinators } \\
\text { Determine difference between } \\
\text { nurse and non-nurse } \\
\text { coordinators } \\
\text { Identify qualifications and role } \\
\text { preparation }\end{array}$ & $\begin{array}{l}\text { Study coordinators learn about gene therapy research 'on } \\
\text { the job', by physicians and lack formal training } \\
\text { Nurse coordinators were not certified in clinical research } \\
\text { trial management } \\
\text { Perceived high degree of collaboration between } \\
\text { coordinators and PIs } \\
\text { Top ranking priority skill identified as 'protection of } \\
\text { research participants' }\end{array}$ \\
\hline $\begin{array}{l}\text { Bevans et al. } \\
\text { (2011) }\end{array}$ & USA & Survey & $\begin{array}{l}\text { Clinical Research Nurses } \\
(\mathrm{CRN}), \text { Research Nurse } \\
\text { Coordinators (RNC), } \\
\text { Nurse Practitioners } \\
(\mathrm{NP}) \\
(\mathrm{n}=412) \\
\text { Large biomedical research } \\
\text { institution }\end{array}$ & $\begin{array}{l}\text { Describe frequency and } \\
\text { perceived importance of study } \\
\text { activities } \\
\text { Delineate differences between } \\
\text { CRN and RNCs }\end{array}$ & $\begin{array}{l}\text { Two distinct roles identified (CRN and RNC) } \\
\text { CRNs worked more in the 'clinical practice' domain and } \\
\text { significantly less in 'study management', 'care } \\
\text { coordination', subject protection', and 'contributing to } \\
\text { science' } \\
\text { RNCs more focussed on 'coordinating' patient care and } \\
\text { research activities and tended to be linked to one study or } \\
\text { PI }\end{array}$ \\
\hline $\begin{array}{l}\text { Catania et al. } \\
(2012)\end{array}$ & Italy & $\begin{array}{l}\text { Survey } \\
\text { (CTNQ) }\end{array}$ & $\begin{array}{l}\text { Clinical Trials Nurses } \\
(\mathrm{CTN})(\mathrm{n}=30) \\
\text { Oncology }\end{array}$ & $\begin{array}{l}\text { Assess the role of CTN } \\
\text { Evaluate the quality of the job } \\
\text { performed }\end{array}$ & $\begin{array}{l}\text { Italian CTNs mostly focussed on trial activities, including } \\
\text { 'consent process', 'implementation and evaluation of } \\
\text { protocol' and 'investigational product management' }\end{array}$ \\
\hline
\end{tabular}




\begin{tabular}{|c|c|c|c|c|c|}
\hline Reference & Country & Method & $\begin{array}{c}\text { Participants and } \\
\text { setting }\end{array}$ & Research aim & Findings in relation to this study \\
\hline $\begin{array}{l}\text { Davis et al. } \\
\text { (2002) }\end{array}$ & USA & Focus Group & $\begin{array}{l}\text { Study Coordinators } \\
\text { (nurses, } \mathrm{n}=31 ; \\
\text { non-nurses, } \mathrm{n}=14 \text { ) } \\
\text { Academic medical centre, } \\
\text { federal research centre, } \\
\text { private organisation }\end{array}$ & $\begin{array}{l}\text { Compare whether there was a } \\
\text { difference in protection of } \\
\text { research subjects, depending on } \\
\text { workplace }\end{array}$ & $\begin{array}{l}\text { Study coordinators balance } 3 \text { advocacy roles; patient, } \\
\text { subject and study } \\
\text { Some difference found between the balance of the } 3 \\
\text { advocacies depending on the workplace }\end{array}$ \\
\hline $\begin{array}{l}\text { Duane et al. } \\
\text { (2007) }\end{array}$ & USA & Survey & $\begin{array}{l}\text { Clinical Research } \\
\text { Coordinators }(\mathrm{CRC}) \\
\text { (nurses, } \mathrm{n}=101 ; \\
\text { non-nurses, } \mathrm{n}=104 \text { ) } \\
\text { Members of Association } \\
\text { of Clinical Research } \\
\text { Professionals (ACRP) }\end{array}$ & $\begin{array}{l}\text { Describe the CRC work tasks, } \\
\text { percentage of time spent on each } \\
\text { task and perceptions of CRC } \\
\text { work experiences }\end{array}$ & $\begin{array}{l}\text { On the job training }(56 \%) \\
\text { Obstacles identified as multi-tasking, recruitment, } \\
\text { interruptions and inadequate compensation }\end{array}$ \\
\hline $\begin{array}{l}\text { Gordon } \\
(2008)\end{array}$ & UK & Not stated & $\begin{array}{l}\text { Clinical Research Nurses } \\
\text { (CRN) (not stated) }\end{array}$ & $\begin{array}{l}\text { Pilot data about amount of time } \\
\text { spent on various study tasks }\end{array}$ & Majority of time spent on clinical tasks and administration \\
\hline $\begin{array}{l}\text { Hill \& } \\
\text { MacArthur } \\
\text { (2006) }\end{array}$ & UK & $\begin{array}{l}\text { Survey (2) } \\
\text { Focus Group }\end{array}$ & $\begin{array}{l}\text { Research Nurses } \\
\text { (Study } 1, \mathrm{n}=72 ; \text { Study } 2 \text {, } \\
\mathrm{n}=50, \text { Study } 2 \mathrm{a}, \mathrm{n}=16 \text { ) } \\
\text { Lothian University } \\
\text { Hospital National Health } \\
\text { Service Trust }\end{array}$ & $\begin{array}{l}\text { To develop a profile of research } \\
\text { nurses working for the Trust } \\
\text { Explore professional issues } \\
\text { related to their role }\end{array}$ & $\begin{array}{l}\text { Most on fixed term contracts }(65 \%) .72 \% \text { had job } \\
\text { descriptions, half of which were accurate. Line manager: } \\
\text { doctors }(43 \%) \text {, directorate manager }(40 \%) \text {, nursing } \\
\text { manager (17\%) } \\
\text { Majority reported 'isolation'. Concerns raised regarding } \\
\text { lack of support and being ethically compromised }\end{array}$ \\
\hline
\end{tabular}




\begin{tabular}{|c|c|c|c|c|c|}
\hline Reference & Country & Method & $\begin{array}{c}\text { Participants and } \\
\text { setting }\end{array}$ & Research aim & Findings in relation to this study \\
\hline $\begin{array}{l}\text { Jeong et al. } \\
\text { (2007) }\end{array}$ & Korea & Survey & $\begin{array}{l}\text { Clinical Research Nurses } \\
(\mathrm{CRN})(\mathrm{n}=79) \text { and } \\
\text { Investigators }(\mathrm{n}=71) \\
\text { Hospitals, pharmaceutical } \\
\text { companies and contract } \\
\text { research organisations }\end{array}$ & $\begin{array}{l}\text { Determine the standard roles, } \\
\text { proficiency and qualifications of } \\
\text { CRNs from CRN and } \\
\text { investigator perspective }\end{array}$ & $\begin{array}{l}\text { Important role components identified by both CRNs and } \\
\text { investigators: communication, completing CRFs (data) } \\
\text { and obtaining informed consent } \\
\text { Statistically significant different perception between } \\
\text { CRNS and investigators: } \\
\text { - CRNs > Investigators: 'recruiting subjects' and } \\
\text { 'screening subjects' } \\
\text { - Investigators > CRNs: 'preparing for monitoring } \\
\text { and inspection' and 'scheduling subjects' }\end{array}$ \\
\hline $\begin{array}{l}\text { Loh et al. } \\
\text { (2002) }\end{array}$ & Australia & Focus Group & $\begin{array}{l}\text { Data Managers } \\
\text { (nurses, } \mathrm{n}=14 ; \\
\text { non-nurses, } \mathrm{n}=7 \text { ) } \\
\text { Large teaching hospitals }\end{array}$ & $\begin{array}{l}\text { Explore views of data managers } \\
\text { and compare their role to } \\
\text { physicians in obtaining consent }\end{array}$ & $\begin{array}{l}3 \text { roles identified in informed consent process: } \\
\text { - Information provision } \\
\text { - Quality assurance } \\
\text { - Ongoing support during the trial }\end{array}$ \\
\hline $\begin{array}{l}\text { Mori et al. } \\
\text { (2007) }\end{array}$ & USA & $\begin{array}{l}\text { Survey } \\
\text { (CTNQ) }\end{array}$ & $\begin{array}{l}\text { Clinical Research Nurses } \\
(\mathrm{CRN})(\mathrm{n}=109) \\
\text { General Clinical Research } \\
\text { Centres (GCRCs) }\end{array}$ & $\begin{array}{l}\text { Describe the role of CRN to } \\
\text { inform development of CRN } \\
\text { professional organisation and } \\
\text { certification programme }\end{array}$ & $\begin{array}{l}\text { CRNs primarily focussed on clinical implementation of } \\
\text { trials. } \\
\text { Recommended standardising through certification to } \\
\text { promote consistency in education, adherence to standards } \\
\text { and professionalism. } \\
\text { CRNs had high job satisfaction, autonomy, but also stress }\end{array}$ \\
\hline
\end{tabular}




\begin{tabular}{|c|c|c|c|c|c|}
\hline Reference & Country & Method & $\begin{array}{c}\text { Participants and } \\
\text { setting }\end{array}$ & Research aim & Findings in relation to this study \\
\hline $\begin{array}{l}\text { Mueller } \\
(2001) \\
\text { Mueller \& } \\
\text { Mamo } \\
(2000) \\
\text { Mueller \& } \\
\text { Mamo } \\
(2002)\end{array}$ & USA & Interviews & $\begin{array}{l}\text { Nurse Trial Coordinators } \\
\text { (Study } 1, \mathrm{n}=32 ; \text { Study } 2, \\
\mathrm{n}=24, \text { Study } 3, \mathrm{n}=24 \text { ) } \\
\text { Hospital-based medical } \\
\text { centres (public and } \\
\text { private clinical trials) }\end{array}$ & $\begin{array}{l}\text { Examine work of nurse } \\
\text { coordinators and occupational } \\
\text { processes. } \\
\text { Career contingency } \\
\text { Job satisfaction }\end{array}$ & $\begin{array}{l}\text { Trial coordination activities delegated 'down' from } \\
\text { doctors to nurses (sociological context) } \\
\text { Development of the role, and the way nurses became trial } \\
\text { coordinators varied } \\
\text { Identified positive aspects as more in-depth relationship } \\
\text { with patients, enhanced relationship with physicians, } \\
\text { clinical skill and knowledge, and autonomy } \\
\text { Negative aspects were uncertainty about funding, } \\
\text { professional isolation, workload stress, paperwork, too } \\
\text { specialised and lack of recognition on publications }\end{array}$ \\
\hline $\begin{array}{l}\text { Nagel et al. } \\
\text { (2010) }\end{array}$ & $\begin{array}{l}\text { USA \& } \\
\text { Canada }\end{array}$ & $\begin{array}{l}\text { Survey } \\
\text { (CTNQ) }\end{array}$ & $\begin{array}{l}\text { Clinical Research Nurses } \\
(\mathrm{CRN})(\mathrm{n}=85) \\
\text { Paediatric oncology }\end{array}$ & $\begin{array}{l}\text { Describe the roles and } \\
\text { responsibilities of CRN }\end{array}$ & $\begin{array}{l}\text { Most common roles identified were consent process, } \\
\text { implementation of study protocol, professional nurse role } \\
\text { performance, data management and protocol planning } \\
\text { High levels of competence, satisfaction but also stress } \\
\text { reported }\end{array}$ \\
\hline
\end{tabular}




\begin{tabular}{|c|c|c|c|c|c|}
\hline Reference & Country & Method & $\begin{array}{c}\text { Participants and } \\
\text { setting }\end{array}$ & Research aim & Findings in relation to this study \\
\hline $\begin{array}{l}\text { Rickard et al. } \\
(2006) \\
\text { Rickard et al. } \\
(2007) \\
\text { Roberts et al. } \\
(2006)\end{array}$ & $\begin{array}{l}\text { Australia } \\
\& \mathrm{NZ}\end{array}$ & Survey & $\begin{array}{l}\text { Research Coordinators } \\
\text { (RC) } \\
\text { (nurses, } \mathrm{n}=46 \text {; } \\
\text { non-nurses, } \mathrm{n}=3 \text { ) } \\
\text { Intensive Care Units }\end{array}$ & $\begin{array}{l}\text { Describe demographics, } \\
\text { education and work experience } \\
\text { of ICU RCs } \\
\text { Describe the current role and } \\
\text { structure of RC positions } \\
\text { Job satisfaction }\end{array}$ & $\begin{array}{l}\text { Mostly female nurses, aged } 30 \text { - } 50 \text { years, with } \\
\text { postgraduate qualifications, working in Level III ICUs } \\
\text { Role content included ethics submissions, liaison with the } \\
\text { healthcare team, data collection, assess patient's condition } \\
\text { and education } \\
\text { Satisfaction about flexibility and hours of work, } \\
\text { autonomy, making a difference to patients, intellectual } \\
\text { stimulation and working with multidisciplinary team } \\
\text { Dissatisfaction about remuneration and on call, isolation, } \\
\text { stress, lack of professional recognition and career } \\
\text { pathway, high workloads, protocol requirements, tension } \\
\text { with nursing and senior management }\end{array}$ \\
\hline $\begin{array}{l}\text { Rico- } \\
\text { Villademoros } \\
\text { et al.2004 }\end{array}$ & Spain & Survey & $\begin{array}{l}\text { Clinical Research } \\
\text { Coordinators (CRC) } \\
\text { (nurses, } n=14 ; \\
\text { non-nurses, } n=23 \text { ) } \\
\text { Oncology }\end{array}$ & Determine standard tasks & $\begin{array}{l}\text { CRCs were mainly involved in trial activities involving } \\
\text { administering the protocol } \\
\text { Most CRCs were extremely or very satisfied with their job }\end{array}$ \\
\hline
\end{tabular}




\begin{tabular}{|c|c|c|c|c|c|}
\hline Reference & Country & Method & $\begin{array}{c}\text { Participants and } \\
\text { setting }\end{array}$ & Research aim & Findings in relation to this study \\
\hline $\begin{array}{l}\text { Roberts et al. } \\
\text { 2011a } \\
\text { Roberts et al. } \\
2011 \mathrm{~b}\end{array}$ & $\begin{array}{l}\text { Australia } \\
\& \mathrm{NZ}\end{array}$ & Survey & $\begin{array}{l}\text { Research Coordinators } \\
(\mathrm{RC})(\mathrm{n}=56) \\
\text { Intensive Care Units }\end{array}$ & $\begin{array}{l}\text { Describe demographics, role and } \\
\text { responsibilities } \\
\text { Map professional development } \\
\text { priorities } \\
\text { Job satisfaction } \\
\text { Compare results to earlier } \\
\text { survey }\end{array}$ & $\begin{array}{l}\text { Mostly female nurses, aged between } 30 \text { - } 50 \text { years, with } \\
\text { postgraduate qualifications. Long-term experience in ICU } \\
\text { research } \\
\text { Maintaining ethical standards highest priority. Highest } \\
\text { ranking study duties: ethics submission, protocol } \\
\text { adherence, screening patients, education, data collection, } \\
\text { regulatory and ethics matters } \\
\text { Satisfaction about autonomy, making a difference, } \\
\text { intellectual stimulation, variability and peer support } \\
\text { Dissatisfaction about work hours, lack of support, } \\
\text { recognition and job security }\end{array}$ \\
\hline $\begin{array}{l}\text { Spilsbury et } \\
\text { al. } \\
2008\end{array}$ & UK & Focus Group & $\begin{array}{l}\text { Clinical Research Nurses } \\
(\mathrm{CRN})(\mathrm{n}=9) \\
\text { Pressure area care trial } \\
\text { conducted in } 6 \text { National } \\
\text { Health Service Trusts }\end{array}$ & $\begin{array}{l}\text { Explores scope and potential } \\
\text { contribution of CRN role to } \\
\text { clinical trials of a nursing- } \\
\text { specific topic }\end{array}$ & 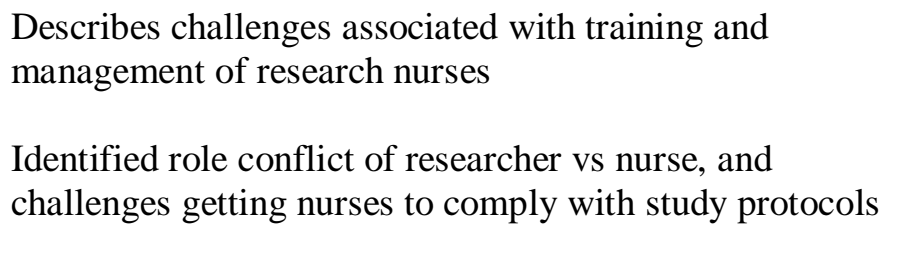 \\
\hline
\end{tabular}




\section{Quality of the literature}

The overall quality of the literature ranged from single centre, pilot studies to large, welldesigned validated surveys. The 'Strengthening The Reporting of Observational Studies in Epidemiology' (STROBE) criteria have been used as one tool in assessing the quality of the literature reviewed (von Elm et al., 2007). The STROBE statement consists of 22 individual components (Appendix A) important to the reporting of observational studies. Each component is either 'present' $(\mathrm{Y})$ or 'absent' $(\mathrm{N})$, with the total number of components present used for rating the quality of the publication (Covic et al., 2009). Using the criteria of a low score ( $\leq 10$ items), moderate $(11-16$ items), or good $(\geq 17$ items) identified in Covic et al., there were one poor, six moderate and 14 articles of good quality. The lower ratings tended to be due to using non-validated questionnaires, not including the method in the title, not stating bias, not outlining the statistical method used and/or not reporting on limitations.

It is acknowledged that the STROBE criteria are better suited to assessing the quality of reporting of research, than the actual research itself (von Elm et al., 2007). However, it has been used here as a helpful way of highlighting potential weaknesses when assessing the literature. Other criteria used were; critiquing of sampling frames, data collection techniques and quality of findings reported which are described throughout the literature review. The STROBE score generally aligned with the overall analysis of the studies.

\section{Development of the research nurse role}

Sociologist and Professor of Nursing, Mary-Rose Mueller, was the lead researcher in a series of studies based on in-depth semi-structured interview data conducted between 1992 and 1999 (Mueller, 2001; Mueller \& Mamo, 2000, 2002). The objectives of these studies were to examine the role of the nurse trial coordinator, the activities they perform and explore their career development (Mueller \& Mamo, 2000) using the conceptual framework of the sociology of occupational development (Mueller).

The interviews were performed in two regions of the USA, using a convenience, snowball sampling approach. Thirty nine interviews were audiotaped, transcribed verbatim and coded with recurrent patterns and themes. The number of participants' interviews used for each of the three studies differed according to the objective of the particular study. Six interviews were not included in the final analysis as the audiotapes were lost or incomplete. 
An identified limitation of the studies was that the interviews were conducted over a long period of time (1992 -1999) (Mueller \& Mamo, 2002).

Mueller (2001) examines the path of nurse trial coordinators being delegated duties by doctors to it becoming a fully-fledged specialty of nursing. She states that tasks which may previously have been performed by doctors for clinical trials were now routinely performed by nurse trial coordinators. Potential contributing factors included an increase in clinical trials, recognition of the randomised controlled trial as the 'gold standard' and physicians becoming too busy with other duties to perform the tasks required in clinical trials. Mueller argues that delegation occurs along socially stratified lines, from higher status occupational groups (doctors) to lower status groups (nurses). She also states that nurses enjoyed the "status enhancement" (Mueller \& Mamo, 2002, p. 38) that this new position had. Nurses embraced the responsibility delegated to them, and frequently acted independently. In some examples of their work in clinical trials, nurses themselves resolved issues which arose with patients on trials; however in situations they were less sure about, they would refer to the principal investigator. Making the decision to resolve independently or in collusion with medical staff highlighted the extensive nursing knowledge, skills and decision-making that experienced nurses make all the time. She recognised that trial co-ordination was a combination of work which was perceived as partly medical and partly nursing. Mueller questioned whether the job of coordinating clinical trials could be fulfilled by occupational groups, other than nurses. She challenged research nurses themselves to prove that their skills and knowledge were more valuable to clinical research than those from other occupational groups.

\section{Role and work of the research nurse}

Publications quantifying and 'delineating' the role of the ResN began to emerge from the USA in the mid-2000s, and several have been published since. These international studies point to a clearly defined role for the research nurse of managing clinical trials and ensuring the study protocol is adhered to, in order to produce good quality research.

Three of the studies examining the role of the ResN used the clinical trials nursing questionnaire (CTNQ) (Catania et al., 2012; Mori, Mullen, \& Hill, 2007; Nagel et al., 2010). The CTNQ was developed by a working group for the Oncology Nursing Society in the USA to 'delineate' the role of the ResN (Ehrenberger \& Lillington, 2004). The literature 
indicates that it was developed in response to Mueller's challenge to produce empirical evidence about the role of the ResN. The process of developing the CTNQ included an extensive literature review, which enabled generation of items for the questionnaire, using the Nursing Role Effectiveness Model as a framework (Irvine, Sidani, \& Hall, 1998). The tool was initially validated by way of an expert panel, focus group testing, reviewed and finalised by the working group. The tool itself consists of 154 items and 12 sections $(\mathrm{H}$. Deininger, personal communication, $21^{\text {st }}$ August, 2010). Respondents were asked to indicate the frequency and importance of the predefined items within their role. When the CTNQ was used to quantify the role of the research nurse, it was criticised as being too long by respondents. This is supported by the low return rate of 39.5\% (Mori et al.) and a high number (19\%) of incomplete surveys (Nagel et al.). Despite this limitation, the CTNQ was subsequently translated into Italian and validated using the same methodology as the original English version (Catania, Poir Ã “, Dozin, Bernardi, \& Boni, 2008. It was used to study the clinical trials nurses in Italy (Catania et al., 2012).

A two part study conducted in a Scottish research trust aimed to develop a profile of the research nurses working in the trust and explore professional issues (Hill \& MacArthur, 2006). The first part of the study used a non-validated postal survey which focussed on employment issues, such as line accountability and job descriptions. A second postal survey explored research nurses' knowledge, professional issues, support and educational opportunities. The focus group explored these issues in more depth.

Rickard et al. (2006, 2007) and Roberts et al. (2006) developed the self-reporting RickardRoberts-Research Coordinator Survey (RRRCS) to conduct a cross-sectional survey of Australian and NZ ICU RCs in 2004. It consisted of 33 questions related to demographics, education, employment history, role structure and role components. It can be assumed that RRRCS was not validated, as no reference to validation is made. The second part of the questionnaire used a modified, previously validated McCloskey/Mueller Satisfaction Survey (MMSS) to assess job satisfaction, and MMSS Importance survey so that comparison could be made between tasks that were done and how important they were. Two open-ended questions asking respondents to list the best and worst things about being an RC were included and are discussed in the job satisfaction section of this review. The survey tool was adjusted slightly and used again in 2009 (Roberts et al., 2011a, 2011b). 
Both the 2004 and 2009 questionnaires were web-based and IRCIG members were invited to participate by email. Reminder emails were sent between three and four weeks, and again at six weeks. At the time of the first survey, the authors considered that most Australian and NZ ICU RCs would have been members of IRCIG. There was a $71 \%$ response rate to the survey in $2004(\mathrm{n}=49)$, of whom 44 were Australian RCs and five were from NZ. The response rate dropped to 54\% $(\mathrm{n}=56)$ when repeated in 2009 and the number of NZ respondents was not specified. The data were collected anonymously, by using an electronic data collection system and descriptive analysis was undertaken.

Over the decade, there was consistency within the literature about the tasks research nurses performed for clinical trials. The most frequently reported components of the role were clinical skills/patient monitoring, managing the study protocol, educating staff and patients/relatives, treatment administration (drug or other), collecting specimens and other research specific data, completing the case report form (CRF), monitoring for and treating adverse events, ensuring informed consent, monitoring of protocol adherence and reporting non-compliance or protocol deviations (Bevans et al., 2011; Catania et al., 2012; Davis et al., 2002; Jeong et al., 2007; Mori et al., 2007; Nagel et al., 2010; Rickard et al., 2006; RicoVillademoros et al., 2004; Roberts et al., 2011b). One UK study categorised the work of the ResN in Newcastle, England citing unpublished pilot data from Newcastle Clinical Research Facility (Gordon, 2008). Research nursing activity was examined for one week and found that $31.3 \%$ of time was spent doing clinical activities such as venepuncture, intravenous cannulation, study visits and study treatment administration. A further $30.6 \%$ of time was spent on administration, with the remaining time divided between other study related activities.

A role component considered significant to the NZ situation is protocol planning and development. Rickard et al. (2006) and Roberts et al. (2011b) reported 67\% and 73\% respectively of study respondents identified protocol development as part of their role. About half of ResNs in four other studies were involved in protocol planning and in three cases it was rated with a high importance although a low frequency (Catania et al., 2012; Hill \& MacArthur, 2006; Mori et al., 2007; Nagel et al., 2010). Nagel et al. conducted a comparative analysis which revealed that the more qualified a clinical research nurse (CRN) was, the more likely they were to participate in protocol assessment, subject recruitment, obtaining informed consent, data management, and performance of the professional nursing 
role. No statistically significant association was found between the number of years worked in clinical research and any role components.

The work of two separate research-related nursing roles was delineated in a recent USA study set in a large research centre (Bevans et al., 2011). This study consisted of a survey based on a validated conceptual framework, which once again rated frequency and importance of tasks performed. Separation between the roles of research nurse coordinator (RNC) and CRN was identified. The focus of the CRN was more about delivering patient care of research participants, while the RNC was more focused on study management, and overseeing of care to research participants. The role of nurse practitioners (NP) was less clear but incorporated educational, administrative and research roles

In the only study that researched the viewpoint of research doctors as well as research nurses about the research nurse role (Jeong et al., 2007), there was a statistically significant difference between the perceptions of investigators and CRNs in four tasks. CRNs rated 'recruiting subjects' $\left(\chi^{2} 4.23 ; \mathrm{p}<0.05\right)$ and 'screening subjects' $\left(\chi^{2} 6.83 ; \mathrm{p}<0.01\right)$ as important components of their role, more than the investigators did. Conversely, investigators rated 'preparing for monitoring and inspection' $\left(\chi^{2} 6.08 ; \mathrm{p}<0.01\right)$ and 'scheduling subjects' $\left(\chi^{2}\right.$ 8.99; $\mathrm{p}<0.05)$ significantly higher than CRNs. The standard deviations were not provided in this publication. The CRNs and investigators both rated 'communicating with investigator/sponsor' and 'completing CRFs' in their top five roles. This Korean study used a non-validated questionnaire, purposeful selection of respondents and weighted the findings of the investigators more highly than the CRNs in its conclusions. However, it highlights the different perception about the role between the investigators and ResNs. Roberts et al. (2011a) also highlighted participant and management perceptions as an area of future investigation.

Communicating with and educating staff was highlighted as a very important aspect of the research nurse role and was identified as a major factor contributing to protocol adherence (Rico-Villademoros et al., 2004; Spilsbury et al., 2008). Some studies described the ResN as being a 'hub' who liaised with investigators, research companies, patients and their families, clinical staff and other clinics or departments (Davis et al., 2002; Rickard et al., 2006). 
Spilsbury et al. (2008) used the opportunity which arose from conducting a large, multicentre randomised controlled trial about pressure area care across six National Health Service Trusts in the UK to study the role of the CRN. They conducted a focus group to explore the experiences of being a CRN, and hear their observations of care related to trial and clinical practice. Out of the potential $16 \mathrm{CRNs}$, nine participated in the focus group. The participants were guided towards the aims of study, with the facilitator encouraging more in-depth discussion of inconsistencies amongst the group. The focus group discussion was recorded, as well as field notes being made, then transcribed verbatim and analysed using QSR NVivo v.2 - a software package for analysing qualitative data. The data were grouped into themes for content analysis which was performed by more than one team member to ensure trustworthiness. One of the challenges the CRNs voiced was ward nursing staff not complying with the study protocol. In some cases if the RC didn't administer the treatment, which in this case was putting the correct mattress on a bed, then it didn't get done. The CRNs reported that staff just didn't understand the trial process. Often relevant paperwork was not completed or was poorly completed, making it difficult to understand things that had happened with regards to the trial.

Managing trial budgets featured as a major role in the latest study by Roberts et al. (2011b), in which $86 \%$ of ResNs were now responsible for this task, compared to only $47 \%$ in their previous study (Rickard et al., 2006). Although few respondents in Mori et al.'s 2007 study about role delineation prepared study budgets on a frequent basis, $72 \%$ rated it with high importance.

Developing nursing research was not generally formal component of the ResN role. Hill and MacArthur (2006) reported that only 15\% were involved in developing studies to examine nursing practice. Of the 61 ResNs not already involved in nursing research, 52\% expressed an interest in doing so and $59 \%$ of those considered that it would be possible. Rickard et al. (2006) reported that 36.7\% $(n=18)$ of RCs were conducting their own studies, although this did not seem to be as part of their role as RCs. A higher proportion of those who were studying towards or already had masters level degrees, conducted their own research. It was not discussed whether the research being conducted was solely for the purpose of attaining a qualification or to answer a research question. 


\section{Ethics, patient advocacy and consent}

Ethics was discussed in several studies, where the ResN held a key role in ensuring that ethical principles were upheld. An important study of study coordinators, of whom $68 \%$ were nurses, by Davis et al. (2002) was set in three different work settings (an academic medical centre, federal research institution and private organisation) in the USA. Seven focus groups were conducted where participants were given different vignettes about the roles and skills of study coordinators, and then study recruitment. Transcripts of the focus groups were coded using NUD.IST version 5. Their findings indicate that study coordinators balance patient advocacy and welfare with the rights and welfare of a patient as a research subject (subject advocacy) and advancing research goals and ensuring that the right patients were recruited and the study protocol adhered to (study advocacy).

Preparation of ethics submissions was considered part of the RC role by $96-100 \%$ of respondents in Rickard et al. (2006) and Roberts et al. (2011b). Respondents in Mori et al. (2007) rated this as a role component which was performed infrequently, however was of high importance. Although other studies cover ethics issues, these were the only studies where ethics submissions were listed as a major role component. The reason for this is unknown, however may be related to differing ethics systems internationally.

Involvement in the consent process was a major component in many studies. In some studies, it was unclear whether ResNs obtained consent or just ensured it was obtained (Catania et al., 2012; Mori et al., 2007; Nagel et al., 2010). Bevans et al. (2011) articulated this as "facilitate the ongoing informed consent/assent process" (p. 424). The terminology about consent changed between the first and second survey of Australian and NZ RCs from "Request consent" (p. 238) in 2004 to "Obtain consent" (p. 264) in 2009 (Rickard et al., 2006; Roberts et al., 2011b). The number of respondents for whom this was part of their role however, remained high at $78 \%$ and $80 \%$ respectively. Another Australian study conducted a focus group about ethical issues for data managers, two thirds of whom were trained nurses (Loh et al., 2002). The data managers had a role in obtaining consent, and rated upholding ethics by ensuring that consent was informed as important. Hill and MacArthur (2006) highlighted a lack of training to obtain informed consent, even though this was part of the ResNs' role. 


\section{Job satisfaction}

Nine of the 21 studies examined job satisfaction of ResNs as a component of a larger study. Most reported that ResNs were generally very satisfied with their role. The long period of time ResNs tended to stay in their positions was also construed as an overall indicator of job satisfaction (Mueller \& Mamo, 2002; Roberts et al., 2011a). Two UK-based studies reported more negative components than positive, highlighting in particular the sense of isolation ResNs experienced (Hill \& MacArthur, 2006; Spilsbury et al., 2008).

Two studies used the previously discussed CTNQ tool to assess job satisfaction (Mori et al., 2007; Nagel et al., 2010). The CTNQ contains a section titled 'professional nursing role perception", and asked respondents to rate 10 statements about stress, satisfaction, competence, recognition and support on a five point scale (Strongly Agree, Agree, Undecided, Disagree, Strongly Disagree) or "not applicable". Mori et al. reported that the majority $(88 \%)$ of respondents felt competent in their current role, $82 \%$ experienced job satisfaction and 79\% experienced autonomy and independence. These results were reinforced by Nagel et al. (2010) where the majority (94\%) of respondents felt competent, satisfied (90\%) and experienced autonomy and independence (89\%). This was similar to the results of the Spanish Lung Society study in which most (83\%) CRCs expressed job satisfaction and believed they played an important role in oncology clinical trials (RicoVillademoros et al., 2004).

Positive aspects of the role which consistently featured in the studies included autonomy, flexible and social working hours, and intellectual stimulation (Rickard et al., 2007). Mueller and Mamo (2002) analysed the "benefits and drawbacks" of the nurse trial coordinator role, using a sub-sample of 24 from their original 39 semi-structured interviews conducted. Participants identified benefits of the position as autonomy, daytime social hours, different, more intense relationships with patients and a more 'equal' relationship with physicians including more respect from physicians.

Negative aspects of the role included working in isolation, lack of recognition, administration, heavy workloads, stress, and uncertainty about the security of on-going employment in the position (Hill \& MacArthur, 2006; Roberts et al., 2006; Spilsbury et al., 2008). The drawbacks cited by Mueller and Mamo (2002) included lack of recognition on publications, physicians' lack of urgency in dealing with matters, no back-up when trial co- 
ordinator is on leave, position insecurity (grant-dependent), isolation from other nurses and paperwork and the inability to actually change the outcome of diseases such as AIDS in particular patients.

Most significant to NZ, was the job satisfaction of the cohort of 49 Australian and NZ ICU RCs (Rickard et al., 2007; Roberts et al., 2006). The results of the open-ended questions were clustered into four themes; how the job is structured, worth of the job, what the work involves and who I work with. All of these categories had statements for the best and worst aspects. High on the list of best aspects of the role were flexibility and work hours, autonomy, making a difference to patient care, intellectual stimulation and working with the multidisciplinary team. Negative comments included being on-call, poor pay, isolation and stress, lack of recognition, protocol requirements, dealing with ethics committees, workload and nursing colleagues and senior management. There were more positive statements $(60 \%)$ than negative statements $(40 \%)$ in 2004 . When the study was repeated in 2009 , while there were still more positive (52\%) than negative statements $(48 \%)$, the overall findings were more negative than the previous study. The 2009 findings rated 'lack of support or recognition in general' highest in the negative themes, followed by working unsociable hours and lack of job security (Roberts et al., 2011a). The best aspects of the role were autonomy, peer support and variability.

The MMSIS component of the study consisted of 31 points about specific aspects of satisfaction and importance about the role (Rickard et al., 2007). Respondents were asked to select a number from 1-10 with one being not satisfied or important to 10 being very satisfied or important. The questionnaire was formatted so that respondents answered the questions regarding satisfaction, followed by the same points listed for importance. The study found that RCs were generally satisfied with their position with a score of 3.84 (out of 5). The aspects which RCs felt most satisfied with, and had a mean satisfaction score over 4.3 were working social (normal) hours, flexibility, weekends off, level of responsibility and level of control over work. Things that RCs were least satisfied with a level satisfaction level $\leq 3.25$ were compensation for working weekends, child care facilities, other aspects of remuneration package (e.g., no cellphone or laptop provided), salary level and lack of opportunities for career advancement. The open-ended questions were in the middle of the questionnaire, before the predefined points, so to some extent they validated the findings from the questionnaire. 
Rickard et al. (2007) also rated the same role components for importance. Items which rated with the highest importance were level of control over working conditions, level of input into decision making and level of control over work, with $100 \%$ of RCs rating these as very or moderately important. The least important aspects were membership of departmental committees, social contact with colleagues outside work and convenient childcare facilities. The area the authors highlighted as concerning was where an item received a high importance score and a low satisfaction score. The top five of these were compensation for working weekends, salary level, recognition by ICU management, career advancement opportunities and research processes in unit.

A sense of isolation featured in almost every study. Hill and MacArthur (2006) found in one of the questionnaire components of the study that $58 \%$ of ResNs expressed a feeling of isolation within their jobs. The feeling of isolation was reiterated in the focus group component of the study. Focus group participants were also asked for strategies to deal with this sense of isolation. Responses included discussion with clinical colleagues (69\%), contact with other ResNs (48\%), support from manager (14\%) and 10\% found it difficult or could not find a strategy. They also reported that ward nurses didn't understand their feelings of isolation and thought they had an easy job because of the social hours, and didn't see the rest of the job, which in some cases included unsociable hours.

This sense of isolation was also one of the themes that emerged from the study by Spilsbury et al. (2008). The findings of this study were generally quite negative, which the authors attributed, in part, to recall bias as the focus group was conducted after the study had finished. CRNs experienced hostility from ward staff, even though ward management had agreed to participate in the trial. A strategy to overcome this was to help staff in clinical areas to gain their cooperation in research. However, the CRNs experienced role conflict between their role as CRN and their role as a clinical nurse and reported that there was an over-reliance by ward staff to provide clinical expertise beyond the scope of the research project. This included asking staff asking them questions about non-research specific pressure area care. The CRNs in this study also expressed that they were regarded 'differently' to CRNs working on medical trials because their trial was nursing research, although the reason for this was not discovered. 
In contrast, many studies reported support from other staff. In the CTNQ 'perception' section, 66\% experienced acceptance and support from non-research nurses, 85\% support from physicians and 83\% experienced support from administrative staff (Mori et al., 2007). Nagel et al. (2010) reported similar results with $75 \%$ reporting they had support of nonresearch nurses in their role as CRNs, more than $90 \%$ experienced physician support and more than $70 \%$ had administrative support. Nagel et al. reported that CRNs had a dual role of nurse and researcher in the oncology setting. Some of the findings were contradictory such as enjoying learning new skills and knowledge required, but perceiving the specialty as 'too narrow', or being a speciality within a specialty (such as research within the oncology setting) (Mueller \& Mamo, 2002).

A high rate of stress was reported by many ResNs. Mori et al. (2007) reported that $67 \%$ of CRN respondents experienced stress related to their workload. This was even higher in Nagel et al. (2010) at $81 \%$. Stress related to role ambiguity was also reported by $43 \%$ of CRNs in Nagel et al.'s study. Duane et al. (2007) found CRCs considered the number of studies they managed was more than they thought was a reasonable number. Multi-tasking, recruitment, interruptions and inadequate compensation were cited as the four main (over $50 \%$ ) obstacles to completing work tasks. Twenty percent of the respondents in the study stated they worked more than 50 hours per week. This was confirmed by Anderson (2008) who reported $11 \%$ of study coordinators worked 41 - 50 hours per week, and another $11 \%$ working more than 50 hours per week. Heavy workloads were also reported by $24 \%$ of respondents in Roberts et al. (2006).

Many studies suggested strategies to overcome drawbacks of the roles or identified it as an avenue for future research (Mueller \& Mamo, 2002). Some strategies included establishments of a network, establishing a research nurse database and permanent contracts to increase job security (Hill \& MacArthur, 2006).

\section{Role preparedness, career pathways and professional development}

A common theme throughout the literature, from all countries is that ResNs had learnt how to do their work, "on the job" (Anderson, 2008; Hill \& MacArthur, 2006; Mori et al., 2007; Mueller, 2001). Mori et al. reported that $80 \%$ of respondents reporting that they were selftaught. This was backed up by Hill and MacArthur who reported that only $44 \%$ of respondents had been specifically orientated to their research role, although most of the rest 
felt this would have been useful. Ninety percent however, had access to continuing professional development although few took the opportunity to use it.

A significant study examining the role preparedness of gene therapy research coordinators aimed to compare the nurse and non-nurse respondents (Anderson, 2008). This study was a web-based validated survey of study coordinators in the USA. The focus was particularly about ethical preparedness, considering the contentiousness of gene therapy research and likely ethical situations which may arise. The study found that despite study coordinators feeling strongly prepared for their role, few of them actually had formal training in this specialty. Non-nurse study coordinators lacked clinical knowledge, skill and expertise in gene therapy, physiology, disease pathology, pharmacology or adverse events, while the nurse study-coordinators lacked knowledge and experience about research ethics and scientific misconduct.

Research nurses commonly learnt from the Principal Investigators (25\%) and from other study coordinators (25\%) (Anderson, 2008). They also learnt through monitoring visits, by making mistakes, from meetings, and networks. The reasons for learning this way appeared to be partly due to the lack of specific formal training for research nurses and perhaps the lack of career structure (Rickard et al., 2006). Roberts et al. (2011a) built on this theme by examining the RCs' perception of professional development. They found that RCs rated "protection of research participants" (p. 132) as the most important priority for professional development. This was followed by communication skills and organisational, planning and management skills.

According to Mueller and Mamo (2000), career development was determined by a number of related factors, rather than following a predefined career pathway. They called this concept "career contingencies" (Mueller \& Mamo, 2000). Mueller and Mamo (2001) report that there was no single route for nurse to become clinical trials nurses, but rather it was a number of contributing factors which led to this being an advantageous career for a nurse. During the time these interviews were conducted, there was no official training for clinical trials nurses and the nurses had a tendency to "learn on the job" (Mueller, 2001, p. 185). 


\section{Summary and areas for further research}

There was a predominance of literature about the 'tasks' ResNs performed for research trials and a major finding from the literature was that the primary role of the research nurse was the management of clinical trials. The most commonly identified tasks were about administering the study protocol. The 'sociology' studies indicated that study tasks had been delegated 'down' from doctors to nurses, and the work could be seen as both nursing and medical. Was this the case in the ICU setting, where patients are so ill, and families stressed? The Australian literature which was set in ICUs showed a significant number of ResNs were also involved in protocol development - was this role also a feature for NZ ResNs, given the collegial relationship through the organisations identified in Chapter 2 ? While 'identifying study tasks' cannot be identified as a gap in the international literature, it was not known whether NZ ResNs shared this role with their counterparts internationally.

Job satisfaction featured in many studies, with ResNs generally being satisfied with their role. Areas of dissatisfaction identified were: Lack of recognition, poor remuneration, no career pathway, high levels of stress, high workloads, lack of job security and a sense of isolation. Because much was already written about job satisfaction, this shaped the type of questions asked in the ResN interviews, as will be seen in Chapter 4. Isolation was a significant feature for many ResNs internationally. NZ is a small, low populated country with relatively few, geographically distant ICUs who employ a ResN. It was unknown whether NZ ResNs also experienced a sense of isolation, and if so, the strategies they had found to overcome it. The role of ResNs has already been identified in Chapter 2 as relatively new - had that led to a sense of professional isolation? Other challenges faced by the ResNs in NZ were also unknown.

Research nurses tended to be well-qualified and experienced, but undertrained in clinical trial management. The literature indicated that learning 'on the job' was the predominant way of being orientated to the ResN role. Given the newness of the role in NZ ICUs, how did the ResNs learn what to do, and given that there are so few, who taught them? There was little written about the management structures ResNs work within. The two studies which did examine lines of accountability reported that most ResNs reported to PIs, with others reporting to nursing and other managers. It was unknown if there was any similarity amongst who NZ ResNs report to, and the structures they work within compared to other international settings. These same studies identified job security as a problem, and ResNs 
were often employed on a 'contract', rather than as a permanent position. Given the CICM requirements identified in Chapter 2, would this also be the case in NZ ICUs? I had some prior knowledge of funding sources, and together with the literature about job security, I identified this as information which needed to be gathered, when examining the role.

Patient advocacy was a significant feature in only one study (Davis et al., 2002), yet ICU ResNs work in a setting in which patients are extremely vulnerable. Describing the ResN role in balancing the demands of the study with those of the patients, was clearly described in this study. Logically, balancing these demands in the ICU setting could be even more important, given the vulnerable population. This Davis et al. article had a significant impact on my study as I sought to explore whether ResNs also had an advocacy role in the ICU research setting.

A significant feature of the literature was that by far the most commonly used method was a survey design. This finding was important when designing this study because while survey gave an excellent overview of the demographics of the ResNs, and the tasks they performed, it lacked depth. An example was that ResNs were identified as being involved in the consent process, yet it was unclear whether they obtained consent, or just ensured that a consent form was completed by a member of the research team. Additionally, although participation in ethics submission was noted in three studies, this process was not described. The ethics system in NZ is very robust, and whether ResNs are involved is significant.

Much of the literature was set in the USA, where the healthcare system differs greatly from NZ. While the literature set in Australian and NZ ICUs was robust, NZ ResNs accounted for only $10 \%$ of the cohort and their accounts have not been analysed separately. All but one of the studies explored the role of the ResN from only the ResN perspective. It wasn't known if this view aligned with other members of the multidisciplinary team they work with. This had significant influence on the research question and overall design of my study, as I sought to describe the role from the ResN, PI and NM perspective.

Despite the increasing volume and quality of literature about ResNs, two major omissions were identified. These are; 'what is the ResN role in the NZ setting?' and 'what are the perspectives of those they work with?' These findings and unanswered questions lead on to 
the next chapter which describes how this study was undertaken to fill some of these gaps in knowledge. 


\section{Chapter 4 Methodology}

\section{Introduction}

The primary aim of this study was to describe the role of the ResN in NZ Level III ICUs, from the perspective of the ResN, PI and NM. The secondary aim was to describe the structure and funding of the research departments in the context of the ResN role. This chapter commences with describing the methodological approach used to answer the research question and the rationale for choosing the qualitative descriptive approach. The overall study design is then reported in depth, including the method used, use of analysis tools, sample selection and recruitment. The chapter also reports on ethical issues encountered, the process of analysis and how rigour was ensured.

\section{Qualitative Description}

The function of the methodology is the "strategy, plan of action, process or design lying behind the choice and use of particular methods" (Crotty, 1998, p. 3) which will answer the research question. Broadly speaking, the considerations when selecting the methodology for this study included the ability to answer the research question, a rigorous approach, collect good data, feasible to do in the time frame, good recruitment of participants and produce results which would add to the body of literature. The research question could have been answered in a number of ways, and survey and case study methodologies were explored and eliminated before selecting the qualitative descriptive approach. As an insider, the methodological approach also had to be 'safe' for me as I was researching my colleagues, who I rely on for advice and support.

The overarching approach to this study is qualitative description. Qualitative research can be defined as research that explores and seeks to interpret social or human behaviour (Creswell, 2009; Gillis \& Jackson, 2002). There is a focus on verbal rather than numerical data. Accurately defining qualitative description is challenging. Even Sandelowski (2010), who has been attributed as identifying qualitative description as a methodology describes it as "naming that that has no name" (p. 80). Qualitative descriptive studies aim to accurately describe a particular phenomena or aspect of society (Gillis \& Jackson), in this case, the role of the ResN. While the interpretation in qualitative description is less in-depth than other qualitative methodologies, some interpretation is still required (Milne \& Oberle, 2005; Sandelowski, 2000). According to Sandelowski (2000) this lower lever of interpretation 
enables the voice and meaning of the participants to be presented as intended. It reflects the views of the people whose behaviour is being described, through rich verbal data, as well as the interpretation of the data by the researcher (Gillis \& Jackson).

Qualitative description was the best approach to answer this research question because the study aimed to describe the ResN role and understand what was already happening in NZ ICUs. Critical discourse was not required as I did not aim to find which interpretation of the role was the right one but to gain an understanding. This included establishing what was happening in the ResNs' role, who they were, what they do and how they do it. Qualitative description allowed me to describe patterns, see if there was consensus or variability about the role and whether there was shared understanding amongst the key informants and amongst ICUs. If there were differences, what aspects were different and what underpinned those differences? I presumed that the role was shared throughout NZ but I didn't know if all components were shared, or what influenced any differences or similarities.

One methodological approach which was considered was survey design using a questionnaire. A survey approach would have been appropriate for gathering data to describe the role of the ResN and the literature review revealed that this methodology had been used to answer similar questions, with some success (Bevans et al., 2011; Mori et al., 2007; Nagel et al., 2010; Rickard et al., 2006, 2007; Roberts et al., 2011a, 2011b; Roberts et al., 2006). Existing questionnaires (G. Eastwood, personal communication, $6^{\text {th }}$ April, 2010, H. Deininger, personal communication, $21^{\text {st }}$ August, 2010) could have been adapted to incorporate the PI and NM participants. The limitations of the survey method include that the sample size would have been very small for this approach and a poor return rate would have resulted in incomplete data. Poor response rates is a common problem with survey with Gillis and Jackson (2002) reporting that response rates between 50 and $75 \%$ are good. However, even a response rate of $75 \%$ would possibly have meant the question was not fully answered. The ultimate reason a survey design was eliminated was that the findings generated would be too similar to a study conducted in Australian and NZ ICUs by Rickard et al. (2006) using a survey methodology.

Case study was also considered as a way of reporting about the role in each ICU. Case study is defined as an in-depth analysis of a case (Stake, 1995; Tellis, 1997). The single case can refer to a "program [sic], event, activity, process of one or more individuals" 
(Creswell, 2009, p. 13). Case study is most useful when you know little about a group or community. Studying 'role' as a phenomena in case study would have been difficult because the information being studied was too general (Stake). Case study would have worked better if I was studying one ResN or one ICU. The issue with case study is that it wasn't going to enable sufficient description about role of the ResN across the ICUs.

\section{Overall study design}

Semi-structured interviews with three sets of key informants were chosen as the overall design. Although usually referred to in ethnography, key informants are those people who know the most about the phenomena (Crabtree \& Miller, 1992; Gillis \& Jackson, 2002). The key informants identified for this study were the ResNs, PIs and NMs. Analysis was planned for within the whole dataset, within key informants' groups and within ICUs. As part of the analysis, a responsibility assignment matrix (RAM) was used to determine who had responsibility for key research tasks. The matrix is discussed in more detail later in this chapter.

\section{Semi-structured interviews}

The data were gathered via semi-structured interviews. Qualitative research has an emphasis on verbal descriptions and the most commonly used method for obtaining such data is the semi-structured interview (Whiting, 2008). Semi-structured interviews are oneto-one interviews which are conducted with some pre-defined open-ended questions, whilst also allowing for themes to emerge (DiCicco-Bloom \& Crabtree, 2006). They differ from other forms of interview in that unstructured interviews are usually used in conjunction with other data collection methods and are non-directive in nature (Ryan, Coughlan, \& Cronin, 2009). Structured interviews have an explicit interview schedule which asks the same question in exactly the same way to all participants (Ryan et al.).

Semi-structured interviews were decided as the most appropriate method for this study because it was possible to direct the course of the interview to gather data needed to answer the research question. It also allowed the participants to initiate other topics and expand on specific things if they wished. There were also other, more pragmatic reasons for conducting interviews including the increased likelihood of participants to keep a prearranged appointment for an interview than respond to a questionnaire. Given the likely sample size, semi-structured interviews of the three participant groups were considered feasible and likely to elicit enough data to describe the role of the ResN. 
One of the considerations, which never arose, was that not all participants would agree to being interviewed. While it was already known that the ResNs were enthusiastic about the study and keen to participate, the likely reaction of PIs and NMs was initially unclear. The nature of this design meant that a comprehensive perspective from each of the sites was required. To off-set the risk of low recruitment of PIs and NMs, a more in-depth interview schedule for the ResNs was developed.

Prior to commencing the interviews, a pilot interview was conducted in April 2011 with a nurse who had previously worked as an ICU ResN, to test the ResN interview schedule and recording equipment. Their feedback was sought and no significant changes were made to the schedules. It was not thought necessary to test the PI and NM interview schedules as they were derived from the ResN schedule which had the most detailed questions.

\section{Other methods considered}

Other methods that were considered included focus groups, document review and selfobservation by way of a time-and-motion diary and a combination of these. Focus groups are a form of interviewing or group discussion where a group rather than an individual is interviewed (DiCicco-Bloom \& Crabtree, 2006). Focus groups are facilitated by a moderator (Plummer-D'Amato, 2008), who relies on the group interacting with each other to elicit rich "interactive data" (Lambert \& Loiselle, 2008, p. 229). The moderator needs to encourage participants to talk about the topic with each other, rather than using probing questions that might be used in individual interviews (Gillis \& Jackson, 2002). An advantage of focus group as a method is the perceived ease of only conducting one 'interview'. However, for the purposes of this study, two main limitations were identified. The ResNs worked in six different ICUs around NZ which would have made it logistically difficult to get them together. Secondly, in focus groups, strong-willed individuals may influence the group discussion and therefore the data gathered (Gillis \& Jackson). While I am unable to say whether this was a possibility for the sample I had selected, I knew that the ResNs had varied levels of experiences and length of service. It was important to capture all of their views and it was possible that more experienced ResNs could dominate a focus group. I was uncertain that as a novice researcher and interviewer, I would be able to manage this situation. 
A time-and-motion study would have been an appropriate method to gather some of the data required (Harrison \& Nixon, 2002; Lindquist et al., 2011) and I considered asking the ResNs to keep a diary of their work activities, hourly. An advantage of this method is that it would have given an accurate reflection of the work ResNs did for that timeframe. It was my own personal experience which eventually discounted the idea. As part of my role, I have to allocate hours to particular clinical trials and projects which is a task I find burdensome and sometimes time-consuming. I also find it disruptive to complete at the time, and often do it retrospectively. Asking ResNs to account for every hour over the period of one or two weeks was therefore considered to be unrealistic given their usually very busy job.

Conducting a document review of the ResNs' position descriptions was also considered to complement the interviews. When I was developing my own position description, I found it very difficult to obtain position descriptions from other hospitals, as they are confidential to the hospital. To replace this, and partly address this issue, a question was included in the ResN interview schedule asking if they had a position description and whether this was indicative of the work they did.

\section{Interview schedules}

Interview schedules were developed for the three participant groups. This was necessary because I recognised that each group would have different levels of knowledge about aspects of the role. The ResNs' interview schedule was the most detailed, incorporating questions about all aspects of the role, finances and the structures of the departments (Appendix B). The PI interview schedule focussed on finances, structures and their understanding of the roles and responsibilities of the ResN (Appendix C). In addition to this, more focus was placed on nursing management in the NM interview schedule (Appendix D).

The interview schedule is recognised as a key element in successful data collection through semi-structured interviewing (Baumbusch, 2010; DiCicco-Bloom \& Crabtree, 2006; Ryan et al., 2009). The progression of the interview schedules was designed to establish rapport with the participants by initially asking closed ended, non-threatening questions before progressing to more open-ended questions about structures and various aspects of the ResN role. The NM and PIs were not well known to me so establishing rapport was important for me as well as the participants. 
All of the interview schedules began with an introduction and then consisted of five sections which aligned with a mixed analysis approach. These sections were background, accountability and funding, ResNs role and responsibilities, processes around studies and future development of the ResN role. The order of the sections and questions was adapted for the individual interview schedules, and as the interviews were semi-structured, the order was adjusted, depending on the flow of the interview.

The 'background' section of the ResN schedule consisted of a collection of closed questions including demographic data about their hours of work, length of service, qualifications, and how they were orientated to the role. The PI and NM interview schedules began with closed-ended questions about their role in the research department in relation to the ResNs and clarification of the studies the ICU was participating in.

The accountability and funding section consisted of a series of closed and open-ended questions, with prompts. The data I wanted to elicit from this section was how the ResNs' positions were funded, and what the structure within the department was. The NMs and PIs were also asked more specific questions about the financial situation in the research department, including the mix of studies they participated in. All groups were asked whether the ResNs were seen as 'senior nurses' within ICU, and also about the expectation of them in relation to resource allocation when the ICU was short-staffed. Specific questions were asked about the CICM guideline requiring tertiary level ICUs to fund a research coordinator (CICM, 2010a), and necessity of, and funding for ResNs to attend work-related meetings.

Questions about the ResNs' role consisted of open-ended questions, with a series of prompts which were used if necessary. ResN participants were asked to recall what they did the last day they worked as a ResN. The only prompts they were given was to think about everything they did, including interruptions. They were then asked if this constituted a typical day and if it was, what would be different about an atypical day, and if not, then what a typical day was. This question was included as an alternative to the time-and-motion diary and was used for content analysis as well as to inform the RAM. One benchmark question was included in this section asking all participants about the expectation of the ResN if a clinical emergency arose while they were with a patient in a research capacity. To 
give context to two questions, the participants were told some background information from the literature about patient advocacy and a sense of isolation. Participants were also told that in one study, $4 \%$ of research coordinators in Australasian ICUs were not nurses, then asked if they thought it was necessary for ResNs to be nurses in the ICU setting. When it came to analysis, I realised that one important question which emerged in some ResNs' data had not been asked of all participants. This was whether there was pressure to do further study. For the participants who had asked to review their transcript, I asked them this question in the cover letter accompanying their transcript. For the others I contacted them by email to ask what their highest qualification was, and whether there was any pressure to do advanced study.

The 'processes around studies' section was the most structured set of questions and was designed to populate the RAM. This section consisted of a mixture of open and closed questions. The closed questions were about who does specific pre-defined tasks.

The final section consisted of open ended questions regarding the development of the ResN role, and specific questions about the CTG recommendation that there always be a research coordinator on CTG-endorsed management committees. PIs were asked more specific questions about the future role of the Res $\mathrm{N}$ in regards to management committees. The ResNs and NMs were also asked about their knowledge of a consultation paper about developing a generic competency framework for ResNs in NZ (Walker et al., 2010). The interview was then closed with an invitation to make any other comments.

\section{Responsibility assignment matrix}

Prior to the interviews, I gave a 30 minute presentation about my proposed study to approximately 70 Australian and NZ ResNs and RCs at the IRCIG Research Coordinators Workshop, in Australia, in March 2011. The presentation was about my experience when I was a novice ResN, the background to why I wanted to conduct the study and basic themes of the international literature. Information delivered about the interviews was selective, outlining broad areas of questions, because only some of the potential ResN participants were at this presentation. Much positive feedback was given from the ResNs present, many stating that my story resonated with them. One of the other guest speakers, Dr Andrew Giddy from the Victoria Ethics and Governance Board approached me during a break (personal communication, $10^{\text {th }}$ March, 2011). He suggested using a "RACI Chart" to assess whether the different occupational groups agreed about the actual tasks that ResNs 
performed. RACI is an acronym for the four levels of responsibility; Responsible, Accountable, Consulted and Informed and is one form of a responsibility assignment matrix (RAM). It seemed like a good suggestion and I explored its use on the internet and in project management books (Project Management Institute, 2008; Smith \& Erwin, 2007; Williams, 2008).

RAMs are used in project management as a way of managing tasks and resources for projects (Project Management Institute, 2008). There are several variations of RAM which show levels of responsibility related to activities, decisions or tasks and articulating an individual's role in a project (Smith \& Erwin, 2007; Williams, 2008). As can be seen in Table 5, in RAMs, staff have various levels of responsibility for individual tasks. When RAMs are used prospectively, the purpose is to plan levels of responsibility assigned, in order to allocate resources accordingly (Williams). Using a RAM retrospectively allows the user to analyse problem areas in projects. For example if more than one person has overall accountability for a task, or if no-one has accountability for a task, this may make decisionmaking difficult (Smith \& Erwin).

Table 5 Levels of responsibility in responsibility assignment matrix*

\begin{tabular}{|l|l|}
\hline Level of Responsibility & Description \\
\hline Responsible & The person who actually does the task \\
\hline Accountable & $\begin{array}{l}\text { The person who has overall accountability for ensuring } \\
\text { that a task is carried out }\end{array}$ \\
\hline Consult & $\begin{array}{l}\text { The person who may be consulted for advice or } \\
\text { information (characterised by 2 way communication) }\end{array}$ \\
\hline Inform & $\begin{array}{l}\text { The person who is informed after decisions have already } \\
\text { been made and who may be required to take action as a } \\
\text { result (characterised by 1 way communication) }\end{array}$ \\
\hline
\end{tabular}

*Adapted from "Role and Responsibility Charting”, by Michael J. Smith \& James Erwin, 2007, Retrieved from http://alliancebestpractice.co.uk/downloaddoc/RACI\%20Explanation.pdf on 31 ${ }^{\text {st }}$ March 2012.

I decided to use a RAM as an analysis tool. This was developed prior to the interviews, so that relevant questions could be included in the interview schedule. The individual RAM charts consisted of a list of study tasks on the $\mathrm{Y}$ axis (Figure 2). These study tasks were developed using the literature and personal experience. The $\mathrm{X}$ axis consisted of different ICU health professionals, who I thought would be involved in the study tasks. I left spaces on the Y axis to include study tasks which I hadn't thought of. The spaces were filled in with 'R', 'A', 'C' or 'I' (Table 5), to indicate the level of involvement (if any), the different 
roles in ICU had for study tasks. The responsibilities could also be combined, so if someone had overall accountability for ensuring the task was done, and also did the task, this would be written as ' $\mathrm{A} / \mathrm{R}$ '.

Figure 2 Sample responsibility assignment matrix used for this study

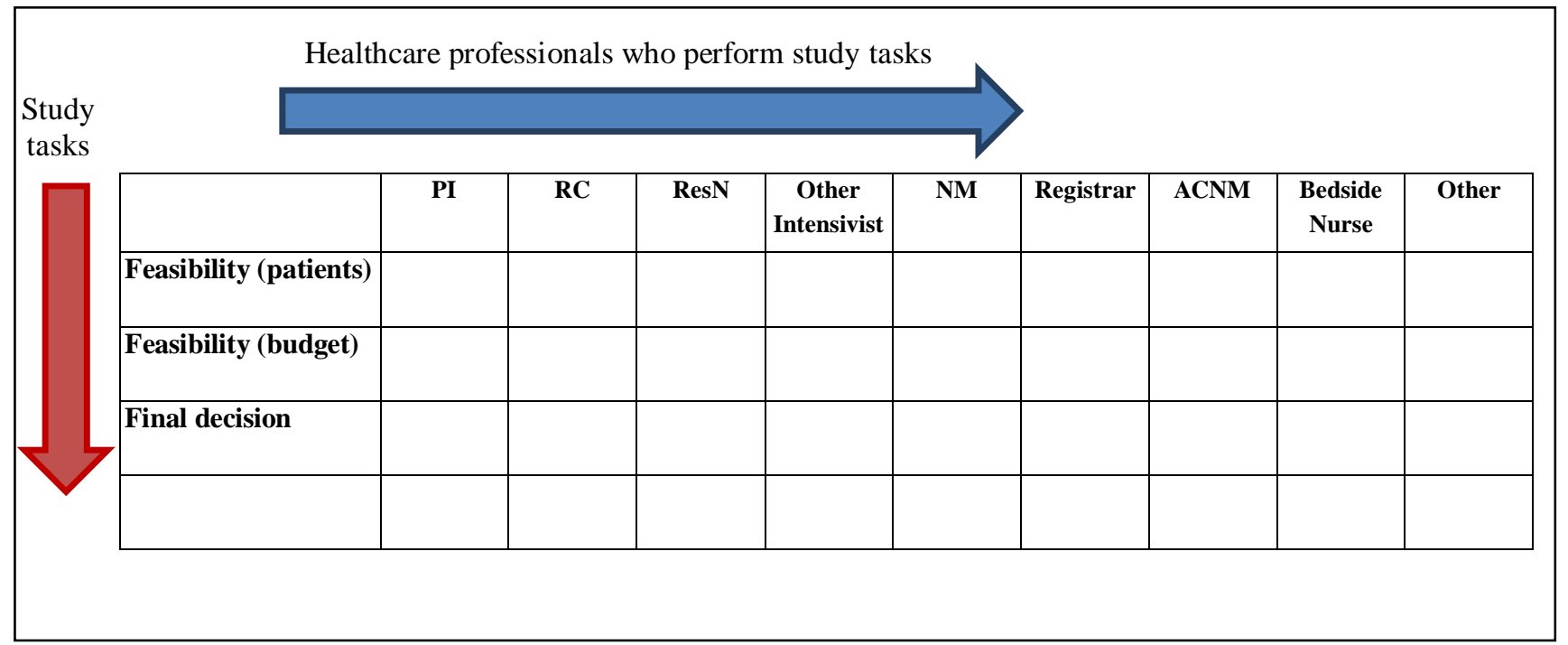

Initially I planned to fill in the RAM during the interview. However, this was too distracting so I introduced the matrix at the beginning of the interviews and explained that part of the interview schedule was directed at completing the matrix afterwards.

\section{Sample selection}

Level III ICUs were selected based on the CICM guideline, and my own knowledge that most of these ICUs employed a ResN. While the line manager for NZ ResNs was not known prior to the study, the literature indicated that PIs and NMs, along with the ResNs, were the most likely to be the most knowledgeable about the ResN role. Including all of these participant groups allowed me to evaluate if there was consensus about the role of the ResN and therefore enable some triangulation. Triangulation is a way of using more than one dataset to corroborate findings and gain a greater understanding of the phenomena being studied (Creswell, 2009; Gillis \& Jackson, 2002).

The inclusion criteria for the study (Table 6) were selected to ensure that the data collected were an accurate reflection of current practice in ICU research. I determined that gathering full data on at least three level III ICUs would be the minimum necessary to describe the ResN's role as I already knew that different ICUs operated in different ways and had different funding structures. I also endeavoured to interview ResNs who had a variety of 
length of service and experience to add depth and richness to the data collected. The PI selected needed to have a current active role in research and a working relationship with a ResN. Articulating this was difficult because some ICUs had several PIs involved in research, while others had one who was in charge of research.

Table 6

Inclusion criteria for participants

\begin{tabular}{|c|c|}
\hline Inclusion Criteria & Rationale \\
\hline \multicolumn{2}{|l|}{ Research Nurses } \\
\hline $\begin{array}{l}\text { Currently employed as a ResN in } \\
\text { an adult Level III ICU }\end{array}$ & $\begin{array}{l}\text { To gather information which is about the current } \\
\text { situation for ICU ResNs in NZ, their knowledge } \\
\text { and experience needs to be current. }\end{array}$ \\
\hline ResN for $>6$ months & $\begin{array}{l}\text { Questions being asked will require ResNs to call on } \\
\text { their experiences and give examples. It is unlikely } \\
\text { they would have sufficient depth of experience in } \\
\text { less than } 6 \text { months. }\end{array}$ \\
\hline \multicolumn{2}{|l|}{ Principal Investigator } \\
\hline $\begin{array}{l}\text { PI for at least one ICU study, or } \\
\text { have been a PI for a study within } \\
\text { the previous } 6 \text { months }\end{array}$ & $\begin{array}{l}\text { The literature determined that most ResNs reported } \\
\text { to an intensivist and it was known from personal } \\
\text { experience and anecdotal accounts that the PI } \\
\text { worked closely with ResNs. }\end{array}$ \\
\hline \multicolumn{2}{|l|}{ Nurse Manager } \\
\hline $\begin{array}{l}\text { Overall management of nursing } \\
\text { staff and budgets in the ICU }\end{array}$ & $\begin{array}{l}\text { Based on the literature and personal experience, an } \\
\text { assumption was made that the ResNs would have } \\
\text { some level of accountability to the NM. At a } \\
\text { minimum, the NM would have knowledge about } \\
\text { the role from managing the nursing staff. }\end{array}$ \\
\hline
\end{tabular}

Eleven ResNs were identified as meeting the criteria of being employed in their position for six months or more. As all 11 ResNs were aware of this study, once ethical approval was gained, they were all approached individually by email inviting them to participate. This invitation was accompanied by the information sheet (Appendix E) and consent form (Appendix F). The information sheet outlined the intent of the study, and positioned confidentiality and identifiability. This email elicited a 100\% agreement to participate, and enquiries were made by phone and email to determine the ResNs' availability for interviews. Because of the logistics of interviewing in six different ICUs, interviews were grouped together where possible. 
I planned to interview all of the ResNs prior to the PIs and NMs. The reason for this was twofold. Firstly, it meant that I could gather information about specific studies the ICUs were participating in, get an overview of the structure and also identify any issues which needed to be explored further in the PI and NM interviews. Secondly, as a novice interviewer, it was easier for me to interview people who were well known to me before the challenge of interviewing senior ICU staff, who I did not know.

The names of PIs and NMs were sought from the ResNs. Asking the ResNs for the contact details of the PI was most appropriate way of selecting a PI for each ICU. This had the potential to introduce some bias however, was not considered a problem as in four cases, the PI selected was in charge of research. Of the remaining two, one ResN selected the PI who was most involved on a daily basis. In the other case, both PIs were considered appropriate by the ResN and I contacted both in advance, with the final decision being made based on their availability on the day of the interview. As each ICU only had one NM, no decision had to be made about appropriateness.

An email of introduction explaining who I was and the purpose of my study was sent individually to all potential PI and NM participants, along with the consent form (Appendix F) and relevant information sheet (Appendix G). All PIs and NMs already knew about the study as they had been informed by the ResNs and all responded that they were agreeable to participating. Further liaison was required to ensure their availability. In some cases, an exact time for the interview was not able to be booked, but the day or days I would be in their hospital was confirmed. A PI and the NM from all six ICUs agreed to be interviewed.

\section{Setting for interviews}

Logistically, the easiest place for the interviews to take place was in the participants' own ICUs. An empty office was sought to conduct each of the interviews. This was their own office for three of the ResNs and all of the PIs and NMs. While it has been suggested that participants choose where the interview takes place (Clarke, 2006), the participants in this study were not seen as vulnerable and there was not thought to be a power imbalance. In fact many of the participants would be perceived as my seniors. 


\section{Interviews}

Interviews were conducted over 11 individual days between May and July 2011. While not ideal to conduct too many interviews in a short space of time, given the location of these, a pragmatic decision was made to conduct up to three interviews a day. The order of ICUs was also pragmatic, based on participant and flight availability. The interviews at my own ICU were conducted last, as I wanted to be immersed in the study, and more skilled at interviewing by the time I interviewed staff who I worked closely with. Because of scheduling, not all interviews were conducted in the planned order but in all cases, at least one ResN in each ICU was interviewed before the PI and NM.

Before the interview commenced, participants were asked if they had read the information sheet, offered the opportunity to have any questions clarified and were then asked to sign the consent form. Most participants stated that they had already read the information sheet, although none had printed it out or signed the consent form. Agreement was sought about clarifying data gathered, and in particular the RAM, after the interview if necessary. A copy of the interview schedule was not sent in advance of the interview.

The interview schedule was used as a guide, and field notes were made on the pre-printed individualised interview schedules throughout the interview. People were asked to provide evidence or examples to demonstrate their views, as opposed to just their opinions. At times, participants pre-empted questions when talking about other issues, in which case the questions weren't asked. The interviews were digitally recorded with two digital recorders and all recorded files were downloaded and backed up on-site. I kept an electronic log of the date, time and length of the interviews.

\section{Difficulties encountered with the interviews}

Only minor problems were encountered with the interviews and were symptomatic of working in a busy environment such as ICU. Twelve of the interviews were interrupted by phone calls $(n=4)$ and/or other staff entering the office $(n=9)$. The interruptions were more common in ResN interviews than the other participant groups (ResN, $n=9 ; \mathrm{PI}, \mathrm{n}=2$; $\mathrm{NM}, \mathrm{n}=1$ ). The interruptions ranged from a staff member mistakenly entering the room to lengthy work-related phone calls. The location of one interview had to be moved after it had started due to other staff needing the office. In one ICU, construction work was taking 
place outside, resulting in poor (but decipherable) recording quality, and the ICU intercom was disconnected in another ICU as it was disrupting the interviews.

Two PIs were only available for a limited time for the interview, due to other commitments. One of these interviews was quite rushed and I made a pragmatic decision to prioritise asking questions I considered essential. The PI offered to meet again at a later time or for me to phone with any additional questions or clarification of answers. As I had obtained all of the key material, this was not considered necessary, and the offer was not taken up.

In another case, the PI was working from home on the day I had arranged to interview them. The ResN organised for this PI to be interviewed by phone. This opportunity was accepted as I did not have plans to return to this ICU and I wanted to ensure the views of all stakeholders in the unit were heard. The interview was conducted by landline using a speakerphone and the interview was recorded and transcribed as the others had been.

\section{Analysis}

In qualitative studies, data analysis begins even as data is being collected (Creswell, 2009; Miles \& Huberman, 1994; Silverman \& Marvasti, 2008). While the data were collected using only semi-structured interviews, a mixed approach was used for the analysis. Several authors talk about a systematic approach to analysing qualitative data (Creswell; Sandelowski, 1995; Thomas, 2006). These processes typically included; data preparation, reading the data 'as a whole', analysing for common themes (coding) and interpretation of the data. I was initially guided by the work of Creswell, who described a six step process. The steps I took for data analysis are shown in Table 7.

\section{Interview transcripts}

The first step after gathering the data was to ensure the data were accurate and presented in a way which could be analysed. The interviews were transcribed verbatim, initially by the researcher $(\mathrm{n}=8)$, and then when the enormity of the task and time involved was realised, a grant was obtained from Victoria University of Wellington to pay a professional transcriber to complete the transcriptions $(n=15)$. The transcriber signed a confidentiality agreement (Appendix H). The transcriptions were then checked for accuracy against the recording. For the six participants who had elected to view their transcripts, these were sent with a cover letter and a relatively short time-frame for review. All but one of the reviewed transcripts were returned with minor changes, and some had additional information. 


\begin{tabular}{|l|l|}
\hline Step & Task \\
\hline 1 & Organise and prepare data \\
\hline 2 & Read through all transcripts as a whole \\
\hline 3 & Extract 'concrete' data from transcripts \\
\hline 4 & Open coding \\
\hline 5 & Synthesis and identification of grouping and patterns \\
\hline 6 & $\begin{array}{l}\text { Re-analyse transcripts for consensus about identified } \\
\text { themes }\end{array}$ \\
\hline 7 & Group data by ICU \\
\hline 8 & Group data by role \\
\hline 9 & Interpretation of data \\
\hline
\end{tabular}

Each of the transcripts was then read through in their entirety to get a sense of likely themes (Step 2). The 'concrete' data, including demographics and background information about the ResNs and ICU structures, were extracted (Step 3). The written transcripts were then read through line by line, and multiple themes identified (Step 4). At this point, the answers to specific questions in relation to the pre-defined topics were analysed. For the first two transcripts, my supervisor also analysed the transcripts line by line. The multiple themes were clustered with similar themes and labelled with a code (Step 5). Where themes were identified in some transcripts, all transcripts were then re-analysed for that specific theme, to see if there was consensus, if there were data which didn't comply and to find a good example of identified themes (Step 6). The data were hand coded and collated using spreadsheets and a notebook to record specific data. The final steps including grouping the data by ICU and by role for triangulation (Steps 7 and 8) and the data were then interpreted (Step 9).

The data for this study were analysed in five distinct parts, which were aligned with the interview schedules and have been analysed in different ways (Figure 3). Although the methodology for this study is qualitative, the nature of the question necessitated collection of some demographic data to provide context to the role. The demographic data about the ResNs and the structures of the individual ICUs required no interpretative analysis and were summarised in table form in the results chapter. 


\section{Figure $3 \quad$ Analysis by section of interview schedule}

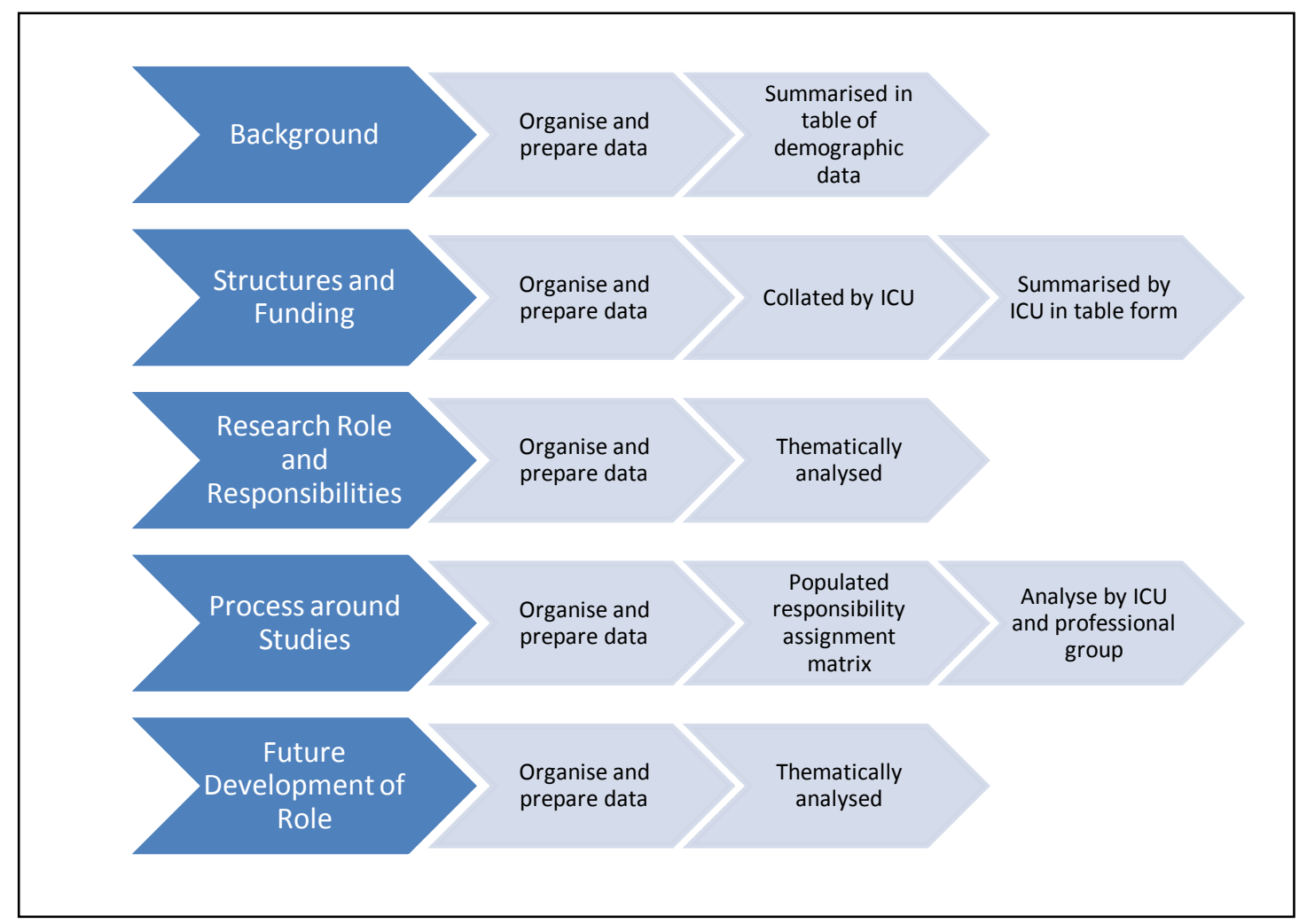

Data about the role of the ResN and future development of the role were analysed thematically. Qualitative data analysis requires familiarity and immersion in the data, and continuous reflection on the data from the time it is gathered (Creswell, 2009; Gillis \& Jackson, 2002). An interpretive approach has been applied to these data. Interpretation of the data involves reading what was said, who said it and what it means.

Defining themes is controversial with DeSantis and Ugarriza (2000) arguing that there is no clear definition of theme. For this study, a theme has been defined as "some level of patterned response or meaning with the dataset" (Braun \& Clarke, 2006, p. 82). Prevalence has been expressed using words such as 'many', 'most' or 'few', and where appropriate, numerically. This approach has been used for this study to add clarity and justification for some findings. The ResNs' transcripts were analysed first, then the PIs and NMs. Each dataset was analysed separately, then triangulation across the whole, then triangulated across the unit (Figure 4). 


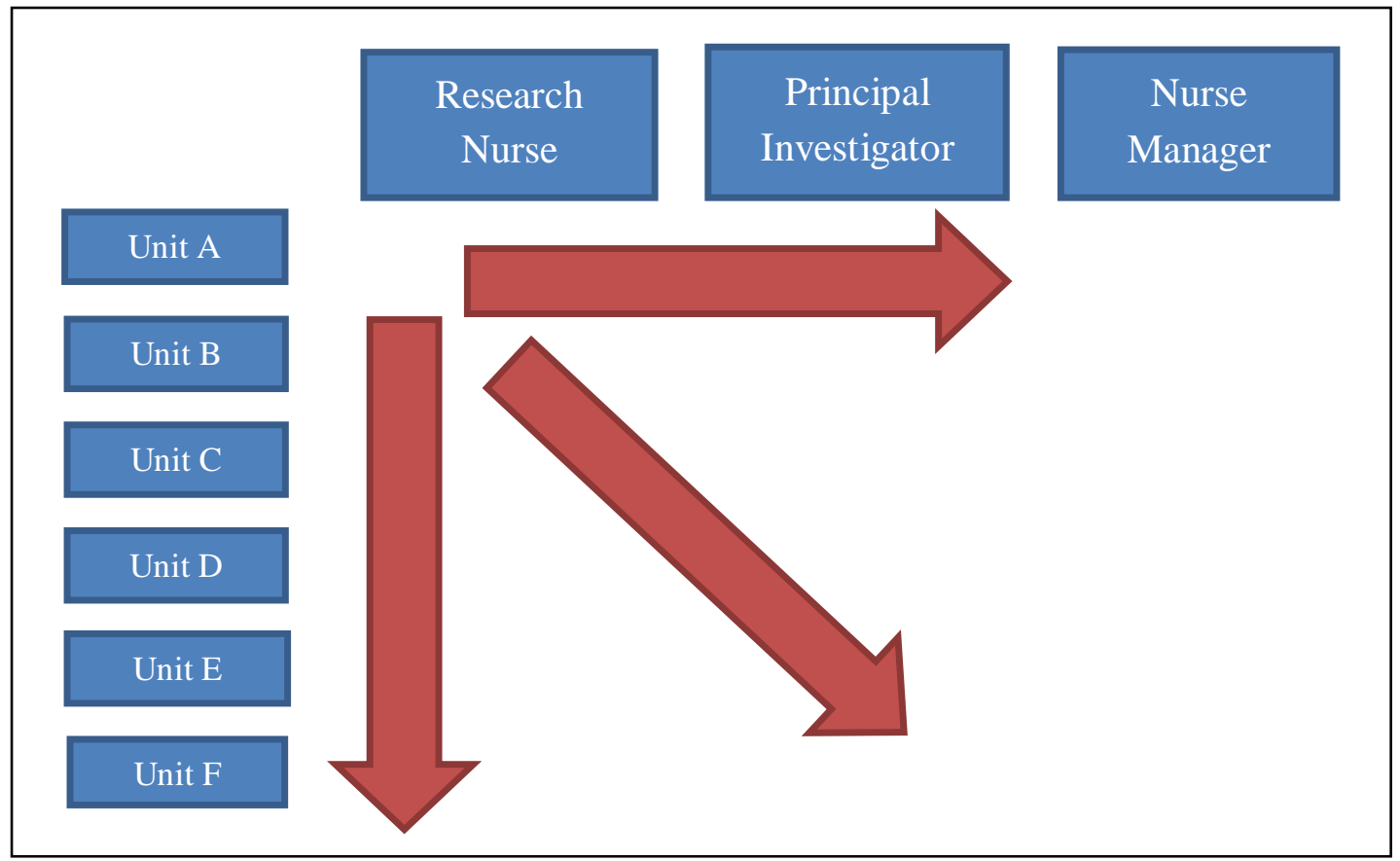

\section{Responsibility assignment matrix}

The research tasks (processes around studies section) were analysed using a RAM chart. The purpose of using the matrix for analysis was to find out what study duties ResNs did, if all of the participants agreed and if the nurses didn't do the tasks, who did? As described earlier, individual RAMs were completed based on the specific and generic interview questions. Once the individual charts had been completed, they were returned to participants as described in the 'member checking' section below.

The individual RAMs were then collated in an excel spreadsheet by ICU, by participant group and then all combined, to see similarities and differences between ICUs and participants (Figure 5). A column for 'Unknown' was added because some aspects of the role were not well understood by some participant groups. The results have not been displayed as a whole, to protect the identities of participants; however, they are referred to in the results section, with examples provided as evidence. To protect the identity of individual ICUs and therefore individual participants, where more than one ResN in an ICU participated in this study, the most common level of responsibility reported is shown. When the analysis was done, all views were recorded. The matrix was amended at the analysis stage, to ensure that all tasks talked about by the participants were captured. 


\section{Figure 5 Sample of analysis using Responsibility Assignment Matrix}

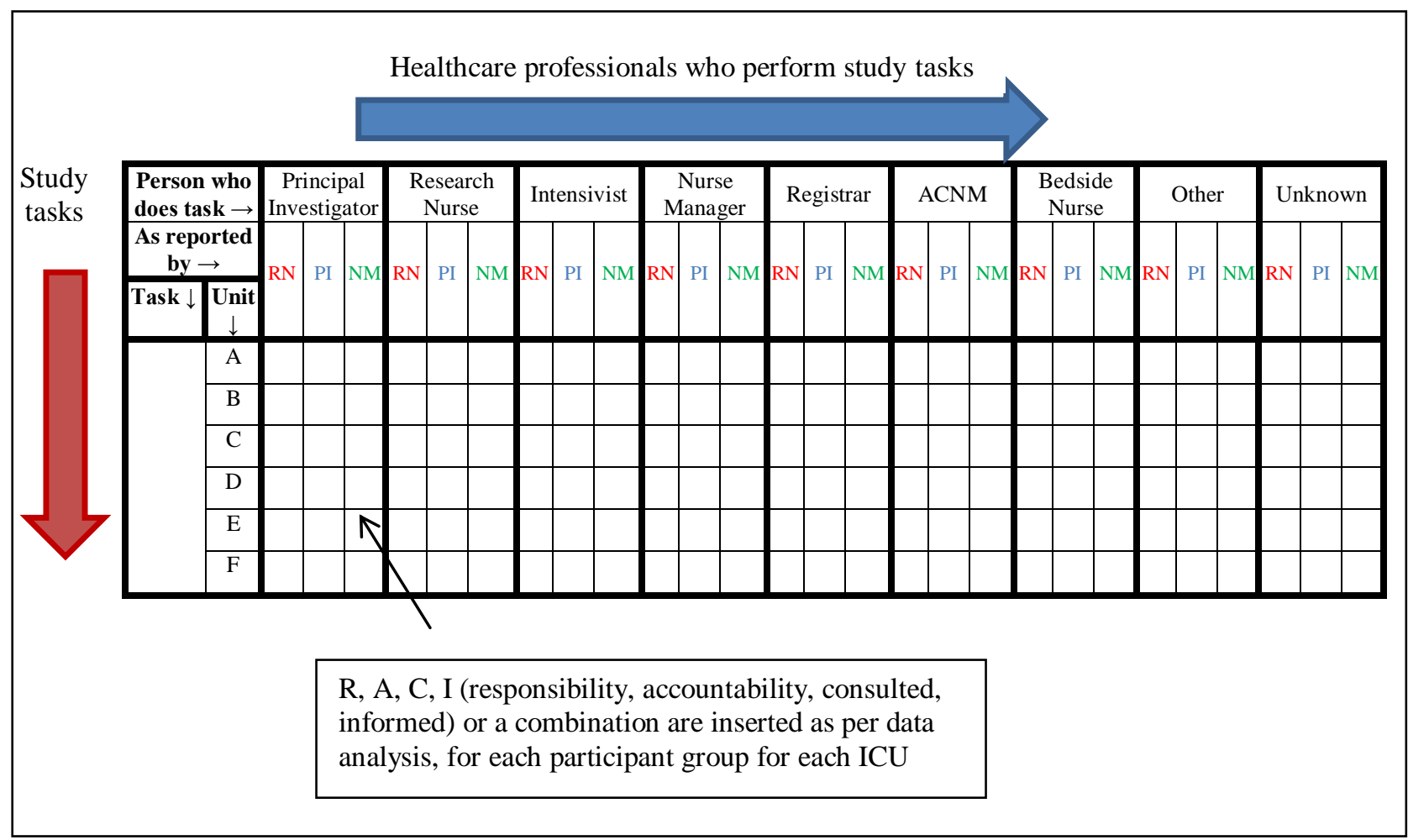

\section{Rigour}

Rigour and trustworthiness are demonstrated throughout this study and are summarised in this section. The purpose of rigour is so that readers can trust the findings of the research (McBrien, 2008; Silverman \& Marvasti, 2008). Rigour strategies employed in this thesis include reflexivity, triangulation, member checking, and audit trail while evidence of data are provided by appropriate use of quotes to give examples of findings (Crabtree \& Miller, 1992; Creswell, 2009; Liamputtong, 2009; McBrien; Sandelowski, 1994)

\section{Reflexivity}

Reflexivity is the process by which researchers have self-awareness of their perspective in the research (McBrien, 2008; Patton, 2002). Recognising how our own values may influence the findings of a study is important for the credibility and rigor of a study (Jootun, McGhee, \& Marland, 2009). Throughout this study, I have had an insider's perspective. I am studying a role I work in, interviewing colleagues I know and interpreting the data myself. At all stages of the study I have endeavoured to put my knowledge and experience 'aside' and rely on the data as presented. This process of 'putting aside' one's own 
knowledge and values about the subject being studied is called 'bracketing' (Silverman \& Marvasti, 2008). As a novice researcher, I need to acknowledge that despite my best efforts, I was probably not able to do this $100 \%$ of the time and this influence would have been present unconsciously throughout the study.

There are advantages and disadvantages to being an insider. Advantages include; access to and trust of the group and shared understanding of terminology (Bishop, 2005). The ResNs and PIs understood my questions, why I was asking those questions and I understood what they were talking about. The disadvantages included; the potential for bias and participants not always giving full answers because they thought I knew what they meant. To overcome this, participants were asked to give examples where possible, or follow up questions and open-ended questions were used. Insider knowledge and bias were not considered problems for this research because this study was about what ResNs do and what participants understood their role to be in research, and their answers were quite 'concrete'.

Despite being an insider, I remained open to interviewees' perspectives. I took the stance when interviewing that no answer was considered right or wrong. I did not challenge people's interpretation of their view of the role; that if a participant said it was true then it was true. During the analysis phase, I did not rank one participant's perspectives above or below others and have tried to present them with equal weighting, unless otherwise reported. The aim of this study was to obtain different stakeholders views, even if they differed from each other's and my own. Likewise, the study was not about testing participants' knowledge about the role. Where appropriate, if I already had information about something factual such as studies they were participating in, this was more of a statement requiring confirmation, than a question in the PI and NM interviews.

To help with bracketing during the interview process, I used the interview schedules with pre-defined prompts. Although it is inevitable with semi-structured interviewing that topics outside of the schedule arise, I endeavoured to follow the pre-defined interview questions where possible. I was careful not to engage in critique or offer information about other people or roles to the participants.

\section{Triangulation}

The term triangulation comes from the fields of navigation and surveying where having more than one landmark assists you to verify your bearings (Gillis \& Jackson, 2002; Patton, 
2002). Triangulation in research allows the researcher to verify their findings by using different data sources, investigators, theories or methodologies (Creswell, 2009; Patton). This study achieved some degree of triangulation by gathering data from the different practitioners, and additionally by analysing data thematically and using the RAM. Where findings from the key informants are incongruent, this has been highlighted in the findings section.

\section{Member checking}

Member checking is the process of checking the accuracy of the data collected with the participants (Liamputtong, 2009). Member checking is generally considered essential to the credibility of data (Creswell, 2009; McBrien, 2008), although Sandelowski (1993) controversially argues that by seeking clarification, the participants may change the data. This was less of a risk in this study, because the interview topic was not controversial, interview schedules were quite structured, examples were constantly sought and participants were unlikely to change their stance.

All participants were offered the opportunity to see their transcripts - four ResNs, one PI and one NM took this opportunity. In all cases, only minor changes were made. For participants who did not wish to view their transcript, I contacted them by email to check data I was unsure of, or that required clarification. Member checking afforded this study the cleanest possible data, and people elaborating or changing their transcripts did not result in significant changes to the final data.

Once the transcripts had been analysed and the information used to populate the individual RAMs, these RAMs were returned to participants for confirmation. This was to ensure the accuracy of my interpretation about study tasks. Of the $14(\operatorname{ResN}=10 ; \mathrm{PI}=2 ; \mathrm{NM}=2)$ who returned the RAMs, 13 made changes, which reinforced the need to member check when determining details about responsibility and accountability on a series of activities.

\section{Audit trail}

McBrien (2008) describes the use of an audit trail as essential for documenting decisions made about data kept and discarded. When the transcripts were initially analysed, notes were made on printed copies of these. Then multiple themes were collated in an excel spreadsheet, and handwritten notebook. The audit trail of information which was kept and discarded has been kept electronically, and thoughts about potential themes were recorded 
in a research diary. Audit trail in qualitative research ensures that even though another researcher may have a different interpretation of the same data, the process by which the findings were arrived at is clear.

\section{Ethical issues}

Ethical approval was sought and obtained from the Victoria University Human Ethics Committee (Appendix I). Several ethical issues were identified in this study. The main issue, which was recognised from the outset, was that the participants were from a select and small group from within the broader ICU community. De-identification will work in the main for people outside of the environment, but given the size of NZ, anyone who is part of the ICU research community may be able to identify the participants involved. Every endeavour has been made for individual quotes to be kept confidential by way of using a randomly assigned coding system for participants. The plural 'ResNs' has been used even if only one ResN was interviewed to prevent identification of the ICU they work in. Tables in Chapter 5 (Findings) have been designed so that cross reference between ResN demographics, ICU structures and quotes is not possible.

Recordings of interviews were only heard by me, and the transcriber, who was bound by a confidentiality agreement. Some written transcripts were viewed by my supervisor to assist with early coding. I have undertaken to keep the recordings and written transcripts confidential and they will be destroyed two years after the completion of this thesis. Care will be taken that any presentations about the results of the study are done in a forum where no one group has privileged information.

Much of the information about the ICUs themselves, such as how many studies they are participating in, was considered to be relatively low risk and readily available to anyone who asked. Information about structures and funding would be relatively easy to obtain within the ICU community and has been discussed in various forms in the public domain.

'Third party critique' was identified as an issue inherent in a study of this kind. The nature of the study topic and the method of semi-structured interviewing meant that participants were talking about other people, or their role, both positively and negatively. While I wanted to hear what people said as it related to the study, I did not take responsibility for the interview to become a 'venting' process. I took a stance in the interviews not to talk about 
individuals or share information and was careful not to engage if participants were critical of other people. This stance was important to make the research safe for everybody involved.

There were two ethical issues which arose at the interviewing stage, which were not anticipated. The first was a desire from many ResNs to know what their particular PI or NM knew about what they did. This was borne out of a feeling that many NMs, in particular, did not know what ResNs did. Obviously this information could not be passed onto the ResNs, but I consider that the findings of this thesis will help inform them, as well as their managers what their role is.

Related to this issue was a desire by PIs and NMs to know how things worked in other ICUs. In most cases, I answered this question with an example of what we do in the ICU I work in. I believe that the findings of this study will enable the information to be presented across ICUs, in a form which PIs and NMs find useful for their own departments. I found this curiosity interesting in such a small community and country where this information could easily be obtained with a phone call or email. I think interviewing the PIs and NMs may have provoked this interest.

One ethical issue which I didn't overly address was that participants may not have had the freedom to decline participation in this study. While I did not coerce people into participating, the ResNs may have felt pressure from their colleagues. It is also possible that when the ResNs told the PIs and NMs about this study, they influenced their decision about participating. This study is not controversial and I am satisfied that participants would have voiced if they did not wish to participate. ICU and research departments in particular, have a philosophy of participating in research so this may have influenced their decisions.

Another ethical issue was that I had to interview my own colleagues, including fellow ResNs, PI and NM who is my line manager. I elected to interview these colleagues last so that my interviewing skills were better than at the beginning. A risk to the data in interviewing colleagues was their desire to give the 'right' answer. At the time of the interviews and on reflection after reading the transcripts, I believe they answered with honesty and integrity. I made a conscious decision prior to the interviews to present myself as a 'researcher' and remain professional throughout the interviews. I believe I achieved 
this but on reflection think it would have been desirable to either omit the ICU I work in, which would have made the data incomplete or engaged another interviewer to interview them. It was challenging interviewing my work colleagues and the interviews were probably more structured, more closely aligned to the interview schedule, and less freeflowing than some others.

\section{Summary}

This chapter has described the methodology and methods employed to conduct this research. Rationales have been provided about these decisions and how they will ultimately answer the research question. The sample and setting have been described and strategies that were employed to ensure the rigour and trustworthiness of the research have been outlined. Ethical issues which were anticipated and encountered have been summarised.

This chapter, along with the preceding chapters will assist the reader to understand the findings which are presented in the next two chapters. 


\section{Chapter 5 Findings: Research participants, departments and supporting structures}

\section{Introduction}

The findings of this research are presented in Chapters 5 and 6 . In Chapter 5, the findings about who the ResNs are, their background and where they work are presented, while Chapter 6 is about the work they do. Specifically, the first part of Chapter 5 introduces the three participant groups. The ICU research departments are then described in terms of the ResN; including the management structures they work within, how their positions are funded, and their functional relationships. Finally the demographics, background and working conditions of the ResNs are reported. While it is unusual to present the findings for the secondary objectives first, it has been done for this thesis, so that the reader knows more about the context of the ResNs' work which is important for understanding the thematic findings presented in Chapter 6.

\section{Research participants}

The 23 people interviewed for this study included between one and three ResNs $(n=11)$, one PI $(n=6)$ and the NM $(n=6)$ from each ICU. The interviews took place between April and July 2011. The mean length of the ResN interviews was 74 minutes (range 50 - 101), the PIs 47 minutes (range 30 - 69) and the NMs was 39 minutes (range 33 - 48).

\section{Research nurses}

All of the ResNs interviewed were female. As seen in Table 8, they had worked in their current ResN positions for a mean of 6.1 years (range 1 - 14), and had worked in an ICU setting for a mean of 17.8 years (range 4 - 33). Two had worked in research prior to their current position. The ResNs' past nursing experience also included working in cardiac care, emergency department, general wards, health education, home care, neurosurgery and paediatrics. They had worked in a variety of positions including charge nurse, duty manager, educator and staff nurse. Several had worked internationally, including Australia, England, Hong Kong, Saudi Arabia and Zimbabwe. 
Table 8 Research nurse participants' demographics*

\begin{tabular}{|c|c|c|c|c|c|}
\hline $\begin{array}{c}\text { Research } \\
\text { FTE }\end{array}$ & ICU FTE & $\begin{array}{c}\text { Time in ICU } \\
\text { Research } \\
\text { Nurse role } \\
\text { (years) }\end{array}$ & $\begin{array}{c}\text { Previous } \\
\text { Research } \\
\text { experience } \\
\text { (years) }\end{array}$ & $\begin{array}{c}\text { ICU } \\
\text { experience } \\
\text { (years) }\end{array}$ & Highest qualification \\
\hline 0.4 & 0.2 & 5.0 & 0.5 & 17 & Post Graduate Diploma \\
\hline 0.4 & 0.6 & 5.0 & None & 10 & Post Graduate Diploma \\
\hline 0.8 & 0 & 9.0 & None & 20 & Post Graduate Certificate ${ }^{* *}$ \\
\hline 1.0 & 0 & 14.0 & None & 33 & Masters (coursework) \\
\hline 0.6 & 0.4 & 1.5 & None & 10 & Masters (coursework) \\
\hline 0.6 & 0 & 1.2 & None & 17 & Post Graduate Certificate ${ }^{* *}$ \\
\hline 0.6 & 0.4 & 7.0 & None & 24 & Post Graduate Diploma \\
\hline 0.5 & 0 & 12.0 & None & 29 & Masters (by thesis) \\
\hline 0.8 & 0.1 & 6.0 & None & 18 & Masters (by thesis) \\
\hline 0.9 & 0.1 & 3.0 & None & 14 & Post Graduate Certificate ${ }^{* *}$ \\
\hline 0.5 & 0.5 & 1.0 & 5.0 & 4 & Post Graduate Diploma \\
\hline
\end{tabular}

*No names or codes have been included in this table because it would make the ResNs readily identifiable when quoted later in the thesis

** Refers to an ICU specific qualification.

\section{Principal investigators}

All of the PIs were doctors (male $n=5$; female $n=1$ ). They had been actively involved in research in their current ICU for a mean of 9.7 years (range 0.3 - 19), and all had experience working with the ResNs on large, multi-centre clinical trials. Three of the PIs had a title as the head of the research department, and a further two considered themselves to be in charge although they didn't have a formal title. Two PIs were also the clinical director of the ICU they worked in.

\section{Nurse managers}

Of the six NMs, five were female and one was male. Five reported that the ResN had some line management accountability to them and in the sixth, the ResN reported directly to the PI. This sixth NM's involvement with research was as part of their ICU research 
management group. Three NMs stated that they were unsure how much they knew about the ResN's role. The reasons given were that the ResNs managed their own research workload $(n=2)$, the NM was relatively new to their position and sorting out research had not been their top priority $(n=2)$ and they were fully occupied with their own operational workload $(n=1)$.

\section{Research departments - structure and funding}

In two ICUs there was not shared understanding, amongst the participant groups, about the structure and management of the research departments. In one of these ICUs, the PI was uncertain who the ResNs' line manager was and thought it may have been the clinical director or administrator, while the ResN and NM thought it was the NM. In the other ICU, there was disagreement amongst the ResNs, NM and PI about overall accountability and professional accountability. Where there was not consensus, I have weighed the evidence and based the displayed models in Figure 6 on the majority view, which in all cases included at least one ResN. This figure shows the four identified models of line management structure within the research departments. The most common line management model was Model A $(n=3)$, with all other models being present in one ICU each. Professional, trial and operational (or financial) accountabilities are shown, based on the participants own descriptions. The bold lines on this figure show the official lines of accountability, while the dashed lines indicate unofficial accountability. In Model D, the day-to-day management of research had been delegated to the PI and research nurse coordinator, who worked collaboratively as a team. Model B shows the ResN has accountability to a service manager, which was identified as a nursing management role.

\section{Structure and lines of accountability}

The ResNs line manager was the NM $(n=8)$, service manager $(n=2)$ or PI $(n=1)$. In addition to this formal line of professional accountability, all of the ResNs had some operational research accountability to the PI. "It's probably officially [NM], but I would only report to [PI] really" ResN01 (L. 506 - 507). This was generally informal and the ResNs reported that they worked more closely with the PI than the NM and subsequently the PI had a better understanding of the role. Three ResNs referred to the PI as 'the boss'. ResN09 found the dual accountability confusing and reported "there's no structure for your job or reporting or management and you lie in-between ... you have two bosses" (L. 213 214). 
Figure 6 Reported line management models for research nurses
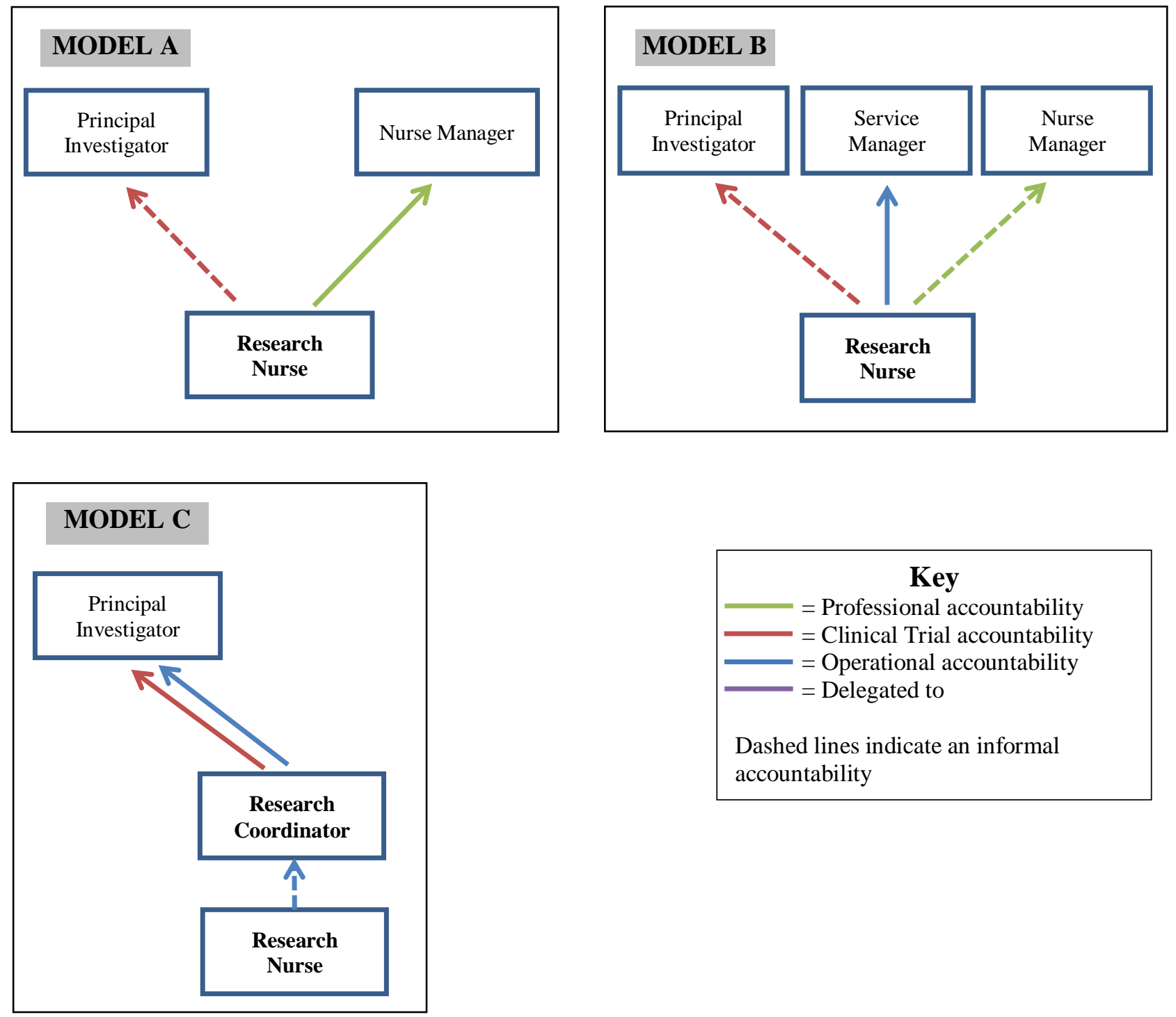

\section{Key}

$=$ Professional accountability

$=$ Clinical Trial accountability

$=$ Operational accountability

$=$ Delegated to

Dashed lines indicate an informal accountability

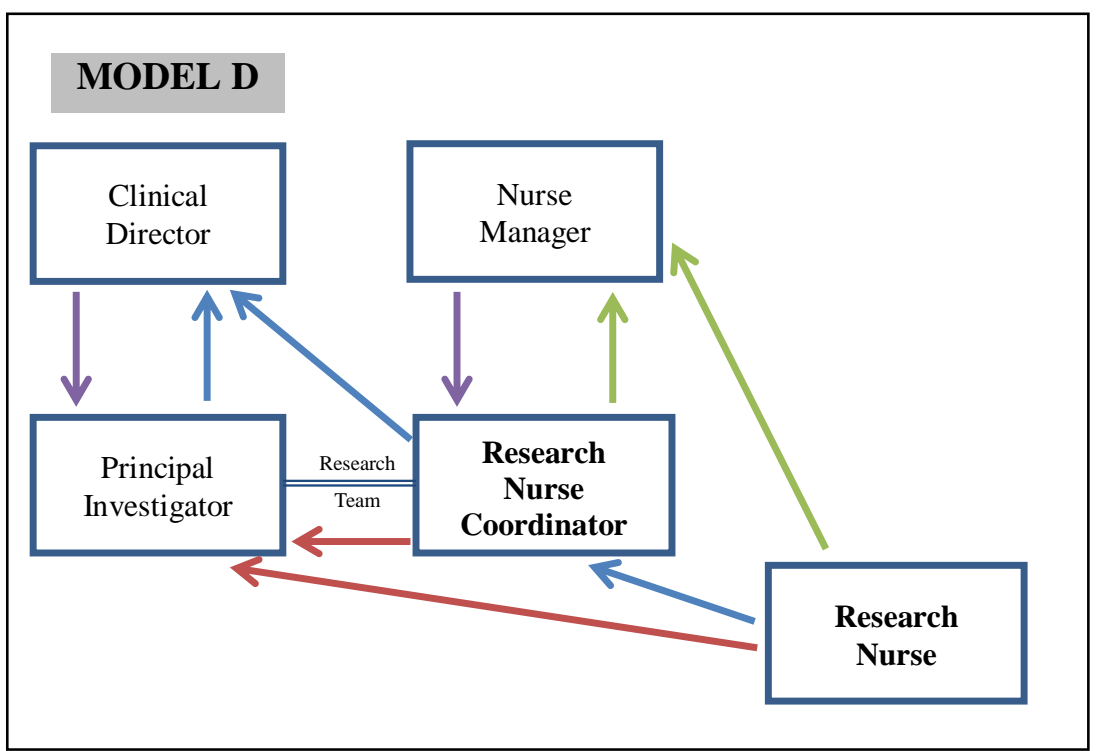


PI04 was unsure whether the ResNs being accountable to the NM was an appropriate structure.

It's a little bit complicated in terms of the staffing because professionally they have to report through the nursing hierarchy, which I have to say is a bit inappropriate I think, but that's the way it is ... and their professional accountability seems to go up through the nursing hierarchy whereas I'm a kind of a business manager I guess for that part of the business rather than the professional manager. (L. 85 - 91)

The ResNs were responsible for the day-to-day research work, and this is discussed further in the next chapter. All of the NMs confirmed this, three with the rationale that the nurses were senior nurses who had been delegated this responsibility and were expected to manage their own workload. NM03 quipped that "no news was good news" (L. 461 - 462).

In five ICUs, there was an intensivist in charge of research from a medical perspective, or who had research as a 'portfolio', in addition to their clinical duties. The remaining PI stated that the PIs in their unit worked as a team and no one in particular was in charge of research. The ResNs $(n=4)$, PIs $(n=5)$ and NMs $(n=5)$ often referred to the ResNs and PIs as 'the research team', who collectively worked together to incorporate research into ICU patient care. In Model D (Figure 6), where the Research Nurse Coordinator (RNC) and PI managed research together, the RNC was still accountable to the PI for study activities which had to be signed by a doctor.

Three ICUs had one main PI who was PI for all of the multi-centre studies. While Table 9 shows that all ICUs have more than one intensivist as a PI for research studies, the majority of the research workload fell to one PI, who had a particular interest, or was 'in charge' of research in ICU. Within these units, other intensivists were the PI for their own studies, which distorts the numbers given in the table. These other studies tended to be small, single-centred studies, which required little or no involvement from the ResN. In two ICUs, all intensivists were sub-investigators for all studies, and took an active role in recruiting patients into studies. More than one intensivist took the lead in the multi-centre studies in three ICUs. 
Table 9 Summary of principal investigators and studies for each ICU

\begin{tabular}{|c|c|c|c|c|c|c|c|}
\hline $\begin{array}{c}\text { ICU } \\
*\end{array}$ & \multicolumn{3}{|c|}{ Number of PIs } & \multicolumn{4}{|c|}{ Number of current studies } \\
\hline & Intensivist & $\begin{array}{l}\text { Research } \\
\text { Nurse }\end{array}$ & $\mathrm{PhD}$ & $\begin{array}{l}\text { Investigator } \\
\text { initiated** }\end{array}$ & Commercial & $\begin{array}{c}\text { Ongoing } \\
\text { Observational }\end{array}$ & Nursing \\
\hline Unit & 2 & 1 & & $8(4)$ & 1 & 2 & \\
\hline Unit & 2 & 1 & & $7(3)$ & 2 & 2 & \\
\hline Unit & 2 & 1 & & $8(2)$ & 1 & 2 & \\
\hline Unit & 3 & 1 & & $1(1)$ & 1 & 1 & 1 \\
\hline Unit & 3 & 2 & 1 & $9(6)$ & 1 & 2 & \\
\hline Unit & 4 & 1 & & $4(3)$ & 1 & 2 & \\
\hline
\end{tabular}

* ICUs have not been given codes in this table so that cross-reference cannot be made when findings are described later in Chapter 5 and 6 in order to preserve the confidentiality of participants.

**CTG-endorsed studies shown in brackets (CTG, 2010a)

\section{Hospital research department}

There had been significant changes to many hospital research departments in recent years and generally the role of these departments was not well articulated. Three PIs described these changes as a rationalisation of trust accounts because in the past, multiple trust funds existed within the hospital, sometimes with large sums of money in them, donated from past patients, or in the case of research, earned from participating in well-funded studies. As a result of these changes, there was variance amongst the participants about whether the hospital research department fulfilled their expectation (ResN; $n=2, P I ; n=3)$ or not $(\operatorname{ResN} ; \mathrm{n}=4, \mathrm{PI} ; \mathrm{n}=0)$. The remaining participants were uncertain either because they had nothing to do with the research department or the department was too new to assess, and one hospital did not have a research department.

The term 'overheads' refers to the costs associated with running a business. In research, it is the amount paid by the ICU to conduct research within the hospital, and contributes to running the hospital research department. As can be seen in Table 10 there was variation in the amount of overhead payments that ICU research departments paid. This is important for ResNs as the financial viability of their position is related to the robustness of their finances. 
Tasks which were identified as involving the hospital research department and would have incurred costs included hospital research office staff, statistician, legal consultation, accountants and Maori consultation. A ResN from the hospital which did not have a research department said they were working towards a system where they were "supposed to actually pay our own overheads" to recompense the hospital for costs incurred.

Table 10 Summary of ICUs' funding streams, research nurse FTE and overheads

\begin{tabular}{|l|r|l|l|}
\hline ICU* & $\begin{array}{c}\text { Research } \\
\text { Nurse } \\
\text { FTE }\end{array}$ & \multicolumn{1}{|c|}{$\begin{array}{c}\text { Research Nurse } \\
\text { Funding }\end{array}$} & \multicolumn{1}{c|}{ Hospital Overheads } \\
\hline Unit & 0.9 & 0.9 FTE studies & 25\% Research Nurses' Salary \\
\hline Unit & 1.0 & $\begin{array}{l}0.5 \text { FTE studies } \\
0.5 \text { FTE ICU budget }\end{array}$ & Nil \\
\hline Unit & 1.2 & 1.2 FTE studies & $\begin{array}{l}\text { Commercial studies } 15-20 \% \text { of income } \\
\text { Grant funded studies Nil }\end{array}$ \\
\hline Unit & 1.5 & $\begin{array}{l}0.5 \text { FTE studies } \\
0.5 \text { FTE ICU budget } \\
0.5 \text { FTE commercial sponsor }\end{array}$ & $\begin{array}{l}\text { Commercial studies 15\% of income } \\
\text { Grant funded studies } 10 \% \text { of income }\end{array}$ \\
\hline Unit & 1.9 & $\begin{array}{l}1.4 \text { FTE studies } \\
0.5 \text { FTE ICU budget }\end{array}$ & $\begin{array}{l}\text { Tiered structure, depending on type of study: } \\
\text { Fixed fee of } \$ 1500-\$ 6500 \text { plus } 0-20 \% \text { of } \\
\text { income }\end{array}$ \\
\hline Unit & 3.2 & $\begin{array}{l}1.2 \text { FTE studies } \\
1.0 \text { FTE commercial sponsor } \\
1.0 \text { FTE grants }\end{array}$ & $\begin{array}{l}\text { Tiered structure, depending on type of study: } \\
\text { Fixed fee of } \$ 1500-\$ 6500 \text { plus } 0-20 \% \text { of } \\
\text { income }\end{array}$ \\
\hline
\end{tabular}

* ICUs have not been given codes in this table so that cross-reference cannot be made when findings are described later in Chapter 5 and 6 , in order to preserve the confidentiality of participants.

** The FTE of ResNs within an ICU shown in Table 10 does not always match the number of ResNs participants listed in Table 8. This is because three ICUs had staff working in research who did not meet the inclusion criteria of working in the position for more than six months. In some cases, they were employed on a short-term basis for a specific trial.

\section{Funding}

Funding is essential for research, and the ability of an ICU to participate in, and produce research relies on funding. This directly affects the ResNs as a large part of the cost of research in ICU is paying staff to manage studies. Table 10 shows that all of the ResNs' positions were at least partially funded by the income from studies and in two ICUs, this was the only income. The 0.5 FTE from the ICU budget in a third ICU, was deemed to be for operational data collection, so the ResN role was actually also funded solely from studies. Two ICUs were additionally funded by an unconditional grant from a commercial 
sponsor and three ICUs had some portion of the ResN FTE funded from the ICU operational budget. NM06 stated that they had only been able to establish the ResN position if it was not funded from the ICU operating budget. This was by agreement with "the CEO [chief executive officer] that it was a cost neutral position that was funded from the research trust fund" (L. 58 - 59), and it had been self-supporting since. Funds for research departments were administered via a trust account, which was kept separate from the ICU operating budget. The trustees were generally a triumvirate consisting of a selection of people including the clinical director, ex-ICU administrators, ex-ICU nurses, ICU administrators, ICU doctors, NM and PI.

Only one PI stated they were fully funded for their research position and had a formal 0.5 FTE research role which was funded by an external commercial sponsor. Another PI said they were allocated $2 \frac{1}{2}$ hours per week for research and the other four managed their research responsibilities around their clinical duties. This lack of time was reported by PI02 as one reason for needing ResNs. "I often find ... my clinical duties just take me away and I wouldn't have time on a daily basis to keep things going” (L. 92 - 94).

As can be seen in Table 9, most ICUs participated in more grant-funded or unfunded investigator-initiated studies, than commercial. All participants were asked whether they preferred a certain mix of studies to maintain the research departments from a financial point of view. Four PIs were pragmatic about the need to do commercial studies and stated they needed to do them to support the rest of the research they undertook. The two who stated they did not need to do commercial studies had ongoing unconditional grants from a commercial sponsor which supported the research positions. They thought this was a good position to be in, because there was no pressure to recruit into studies, or to seek wellfunded studies. Amongst the ResNs, five thought they needed to do a commercial study, and the remaining six thought their ICU had a preference for CTG-endorsed studies. In five of the ICUs, their current commercial study was due to finish shortly after the interviews took place, and all were actively assessing a new commercial study. NMs had a limited knowledge about the different types of studies and four did not know if the fee was a consideration when deciding on studies.

Participants were also asked if they would consider unfunded or poorly funded studies if it was an area of research that the unit was interested in. Five PIs responded that they would 
definitely consider this situation and assessed each study on its merits, and likelihood of improving patient outcomes. Four PIs stated a preference and commitment to CTGendorsed studies, even though these studies only "broke even" at best. PI04 stated that their ICU was "very conscious of cost-recovery" and "had only a limited capacity to do unfunded studies" (L. 169, 179). Six ResNs said they thought their ICU would participate in low-funded studies, with most stating they already were. Many PIs and NMs talked about studies being at least "cost neutral".

\section{College of Intensive Care Medicine guidelines}

Participants' responses to the question that according to the CICM guideline "Level III ICUs have sufficient funding for a full time Research Coordinator to fund an active research programme" (CICM, 2010a) revealed that most of the ResNs thought their position should be at least partially funded. Over half $(n=8)$ thought the position should receive some funding from the ICU, hospital, DHB or government. The remaining three, all of whom worked in research departments funded by an external commercial sponsor, thought it should be funded but didn't know who would fund it. Reasons given for at least partial funding included increased job security $(n=2)$, research being an inherent part of patient care in large Level III ICUs $(n=2)$ and because other senior nurse positions which are part of the CICM guideline, such as nurse specialists were funded $(n=1)$. Most participants however, thought that it was unlikely that DHBs would fund research. There were other benefits to the guideline and ResN08 speculated that it was probably the only reason "we have a research unit here" (L. 975).

Three of the PIs expressed that the research role should be at least partially funded through the DHB. Of the remaining three PIs, two worked in ICUs where the ResN position was already partially funded from the ICU budget. One of these was new to the position and was looking at other sources for funding to support their research department. The other PI stated that the "holy grail is to have enough grant money to fully fund research trials" (PI01, L. 686). PI03 had mixed views about whether funding was a good idea, stating "you lose a bit of autonomy potentially" (L. 1157). Most PIs $(n=4)$ thought it was unlikely that their ICUs would get more or any funding for the research position because "hospitals don't see their role as funding research", or because they were already self-sufficient so they thought hospital management perceived that they didn't need funding. PI03 commented that the CICM recommendation may help an ICU without existing funding for ResNs to "get leverage" but if the role was already supported through other means, it was unlikely to 
help. PI02 stated that it could potentially be used as a "bargaining tool" to get hospitalfunded ResNs. A cited advantage to hospital-based funding was that it provided security for when study recruitment was low. PI01 observed that the recommendation was more about recognising the need for ICUs to participate in research.

I think it recognises that to be a training unit at a top level, part of training is that we can't make progress without doing research, so the trainees need to be exposed to the idea that patients will be involved in research and that it's part of our job to do that and obviously so if you're in this unit as trainee registrars they need to be exposed to that. So I think it is important. And practically you can't do research without [ResNs]. (L. 663 - 668)

Four of the NMs expressed outrage that the intensivists' training organisation would make a recommendation and that it had no financial attachment to it. This was described in various ways including that the guideline "had no teeth" (NM05) and "CICM don't do anything for me" (NM02) and "they [CICM] should fund it" (NM04). Four NMs also expressed that although they would like to see the research position funded there was no surplus in the hospital system, and they were not optimistic about getting funding. NM06 stated that if there was more money available it would be "more likely to go to operational and service budgets" (L. 355) and for NM02 they "would rather have another nurse on the floor than a research person" (L. 262 - 263). NM01 already funded some ResN FTE from the ICU budget and thought that funding was vital to maintain the position.

\section{Sense of security}

Security about the research departments' finances by PIs and NMs was attributed to both existing funds in their trust accounts and ongoing funding streams. In three ICUs neither the NM nor PI were concerned about their finances, and stated they had enough money saved in the trust account to pay the ResNs' salaries for a "long” period of time, even if funding streams were discontinued. One NM said they were not concerned about finances because if study recruitment was too low, one of the ResNs was aware that their tenure would be discontinued.

In the remaining three ICUs, either the PI, NM or both were concerned about the financial situation of their research department. In one of these, the NM and PI were very concerned about ongoing funding and the NM expressed concern that the ResNs may have to reduce 
their hours which didn't give them a sense of job security. The PI stated that they were "always running on empty".

The ResNs' sense of security depended both on the culture of the ICU they worked in and the way their position was funded. Most $(n=10)$ thought that unless their particular funding stream decreased or was discontinued, their position would be secure. Responses ranged from "I don't think my job is secure anymore" to "really secure", with the most frequent response being "as secure as anything [in the DHB] is". ResN09 and ResN11 stated that the research ethos was so strong in their ICUs, particularly among the doctors, that even if their funding ran out, this ethos added security to their positions. In contrast, ResN02 stated although there was always plenty of work to do, the security of their position was dependent on funding being available.

\section{Physical department}

All six of the ICUs had a research department office within the borders of the ICU. Four had a dedicated office only for ResNs, one shared their office with the ICU registrars and the other shared with a nurse educator. The ResNs from two ICUs had only recently moved to the ICU, having previously had their research office located in another department some distance from the ICU. ResN06 reported that being located in the ICU was "much more productive" (L. 24). The most often discussed advantages to having an office within the ICU were "visibility" and feeling like "part of the ICU team". A dedicated research office also provided the necessary space to store the large amount of paperwork that accompanies research, confidentiality, easy access to patient charts and a place to "concentrate".

\section{Relationship of research department to ICU}

Although the research department was physically within the confines of the ICU, where research and ResNs were in the overall ICU structure was less clear. The participants in all but one ICU thought the research department was seen as being slightly separate to the ICU with the most frequently used phrase being "to the side". This finding was as common in ICUs where some of the ResN salary was funded from the ICU operating budget as those where it wasn't. A reason research was seen as separate was because of the financial independence from the ICU operating budget. NM06 articulated this as

we like to think we have a small business that we're running and we're the directors of that small business and we have the final sign off and approval 
for all expenditure and also the vision and direction of the research department. (L. 82 - 85)

There were perceived advantages and disadvantages to being a considered a separate department. The main advantage to this financial independence was that the ResNs hours were protected from short-staffing within the ICU clinical area. Another advantage cited by ResN08 was that the research department was protected from restructuring which had affected other senior nurse positions. The PIs were more likely $(n=5)$ than the NMs $(n=2)$ to say that research hours were protected and PI02 summed this up by saying that "research has to be viewed with some degree of importance" (L. 172 - 173). Of these five PIs, four thought the nurses could help in the ICU if it was short-staffed, only if they chose to and by negotiation. The NMs were more likely to think the nurses should help out if the ICU was busy or short-staffed $(n=4)$ but acknowledged that research was also important and three stated that there were other options such as nurse specialists and educators before the ResN would be called upon. The ResNs were divided on whether they would help in the unit if it was short-staffed. Six stated that they didn't help, four would help and one had never been asked. Reasons given for not helping including separate funding $(\mathrm{n}=3)$, clinical unfamiliarity $(\mathrm{n}=1)$ and "if you started, you would always be helping" $(\mathrm{n}=1)$. Of the four who helped with lunch breaks or short-staffing, they thought it was a valuable opportunity to educate and good for their relationship with clinical staff. They suggested this was important because of the extra workload clinical staff sometimes had because of research.

Negative aspects of being seen as a separate department included a lack of research culture, visibility, ResN credibility, and a sense of isolation or "not being part of the team" (ResN10, L. 18 - 19). Only three ResNs reported feeling isolated, as articulated by ResN09 "So I thought it would be following patients and enrolling patients. I didn't envisage how isolated you'd be and how you're seen as the bottom of the pile. Yep and your manager doesn't understand" (L. 208 - 210). The remaining ResNs stated they did not feel isolated because they felt like they were either part of a team of several ResNs or part of a larger NZ network. ResN02 didn't view professional isolation a problem because she "was ready to step away from the bedside" (L. 930).

This difficulty in positioning where the research department belongs and finding the balance was summarised by ResN08. 
We are a separate bubble in so many ways. And that's quite good because we don't necessarily want to be part of the senior team because of a lot of things that have gone on over the last few years. So it is good to be our own separate bubble; however you don't want to be too separate and you still need to be seen as part of the unit and everything that goes on and - that's one of the reasons that we've really resisted moving offices outside the unit and things like that because we are a part of the unit and if you move too far out then you will be seen as something completely separate. (L. 916 - 924)

\section{Research nurses}

\section{Background}

The ResNs' job titles included research nurse $(n=5)$, speciality nurse research $(n=3)$, nurse coordinator for ICU research $(\mathrm{n}=1)$, research coordinator $(\mathrm{n}=1)$ and research nurse coordinator $(n=1)$. In one ICU, the research nurse coordinator was a position senior to a specialty nurse research position.

Our official hospital title is Research Nurses...It's slowly changing to Nurse Specialist Research... But I put myself down as the Research Coordinator when I'm talking to everyone except patients and families. Because that's what I do - I coordinate research. But when I'm talking to patients and families, I will call myself a Research Nurse, so they know where I'm coming from. (ResN02, L. 23 - 29)

Four of the ResNs in four different ICUs were the first appointed to the ResN role in their ICU. Three were in positions established specifically to assist with clinical trials while the fourth began with a mandate to "get nurses interested in research" (ResN06, L. 236). Generally, the ResN role had developed in response to ICUs or PIs becoming involved in research studies which the PIs did not have time to manage themselves.

There was no single reason nurses became ResNs. Five reported they had been in ICU clinical nursing for a long time and were looking for a new challenge, yet had a desire to stay in the ICU environment. A motivating factor for two ResNs was "getting off night duty" and for another two, the hours and flexibility were appealing. Many $(n=8)$ expressed a prior interest in research through their own tertiary level study or thought it might be interesting because they liked numbers or data, or gaining a greater understanding of critical 
illness. Most ResNs $(n=10)$ stated they still enjoyed the job, citing reasons such as autonomy, intellectual stimulation, variety and 'making a difference' to long-term patient outcomes.

I've stayed in it because I like the autonomy. I like the work. Now that I have a greater understanding and realise that there can be positives and negatives. But I do like it and I do like to think I'm making a difference by doing what the results have shown may necessarily save lives, and I quite like that. And I like the fact that I can help teach people on the floor about research and support them through research. I can do my own research and it's very supported for you to do your own research. (ResN04, L. 241 - 247)

All 11 ResNs had applied for advertised positions, which were generally only advertised within the ICU. ResN04, ResN07 and ResN11 were seconded to the position from their ICU staff nurse position prior to obtaining a permanent position. All but two had not actively sought a position in research, but when the position was advertised, thought it sounded interesting. One of the two ResNs who actively sought a role in research had worked in research prior to working in ICU, while the other had seen ResNs working in ICU.

I have always been a little bit interested in research because I like to know the 'why' and when I started in the ICU there were research nurses and I thought they were so cool. They were doing really cool jobs and ... obviously having saw what they did on the clinical side of things and all the fun stuff didn't actually see all the data collection and the data input and you know all the nightmarish stuff that you have to do or writing ethics applications, all that [stuff] and I thought that would be really cool. (ResN04, L. 209 - 216)

The majority of the ResNs ( $\mathrm{n}=8$ ) had no real understanding or expectation of what the role would entail prior to working in it. The work was busier and more stressful than they expected $(n=4)$, and they hadn't realised the amount of information they would need to know or tasks that weren't seen by other staff, such as ethics applications $(n=4)$. The responsibility weighed heavily on some ResNs and ResN10 reported "Absolutely, it's always there. When you go on holiday you've got stuff building up that's going to have to be done when you get back. It doesn't go away and it keeps me awake at night" (L. 145 147). 
ResNs were either 'self-taught' $(\mathrm{n}=6)$ about their role or learned from more experienced ResNs within their ICU $(n=5)$. Where they were self-taught, they had learned "on the job", through "trial and error" $(\mathrm{n}=2)$, from study monitors $(\mathrm{n}=3)$, reading protocols $(\mathrm{n}$ $=2)$, attending meetings $(\mathrm{n}=1)$ and from ResNs in other NZ ICUs $(\mathrm{n}=3)$. The learning process was described as "a steep learning curve" $(\mathrm{n}=2)$, "felt like a fish out of water" (ResN07) and "thrown in the deep end" (ResN11). Although the PIs were considered supportive, none had assisted with orientating the ResNs. ResNs learnt about GCP guidelines through formal training courses $(n=6)$, in-hospital seminars $(n=2)$ and the remainder through peers, study start up meetings and study monitors.

\section{Qualifications}

The ResNs were well qualified, and all had a post-registration qualification (Table 8). One nurse was currently studying towards a doctorate and a further three expressed that they were likely to work towards a Masters degree in the future. When specifically asked whether there was pressure to undertake advanced study in their role, all but one ResN stated that there wasn't. For ResN09 there was a contractual expectation to work towards a Masters degree because her role was a senior nurse role. ResN04 and ResN08 said although there was no pressure to study, working in the role presented an excellent opportunity to study and they would be well-supported to do so. ResN11 said her PI had offered her support to do her Masters degree. Two ResNs were conducting their own research studies; one towards a $\mathrm{PhD}$, and the other was conducting an observational study, which was not towards a qualification. A third ResN was leading a nursing study about ICU patient communication at her site.

\section{Working conditions}

As shown earlier in Table 8, 10 of the ResNs held part time research positions (0.4 - 0.9 FTE), and one worked full-time. For seven ResNs, their contract was permanent, with a further three due to become permanent shortly after the interviews, while the $11^{\text {th }}$ had a two year renewable contract. All worked variable hours between 0700 - 1800hrs, Monday Friday. Reasons given for working part-time in research were; funding restrictions $(n=2)$, personal preference $(n=2)$, poor pay in research $(n=1)$, and the position had historically been part-time $(\mathrm{n}=1)$. 
In addition to their research role, seven of the ResNs also worked within ICU as a staff nurse $(n=6)$ or charge nurse $(n=1)$. One spent most of her allocated staff nurse time doing the staff roster for her ICU. ResN01 and ResN03 reported that the balance they had between research and clinical was 'just right' and ResN04 would work more in research if it paid better.

There were personal, research and clinical reasons for working clinical shifts. Personal reasons were about remuneration $(n=2)$ that included needing a full-time income $(n=1)$ and better pay doing clinical shifts $(\mathrm{n}=1)$. Clinical reasons were equally divided between maintenance of clinical skills $(n=5)$ and credibility with staff $(n=5)$. Clinical credibility was not always about clinical competence but was described as being important to be "seen" by the bedside nurses. Research reasons were that working clinical helped with recruitment for studies $(n=2)$, increased research profile $(n=2)$, funding restrictions $(n=2)$ and compulsory part of contract $(\mathrm{n}=1)$. "Good for the staff to see you out there doing the stuff as well, not just always sitting in an office. And you find heaps more patients when you're on the floor, than when you're sitting in your office" (ResN01, L 30 - 32).

The four ResNs who did not work clinical had worked in the research role for a mean of 9.6 years (range 1.2 - 14). While all acknowledged that working clinically had advantages, the reasons they didn't included; they had worked in ICU as a nurse for so many years and saw no need to continue doing clinical $(n=4)$, the research job was busy enough $(n=2)$ and it was logistically difficult to fit in $(n=2)$. Three of the ResNs who didn't work clinical had initially worked both, but as the workload for research increased, they found it increasingly difficult to balance the requirements of both jobs. These three all thought it was good to have someone on the research team, who still regularly worked clinically.

Working clinically had its problems. When ResNs worked as bedside nurses, most reported doing research duties such as screening for potential patients and enrolling them into studies, while also having sole care of a critically ill patient. ResN02 commented that she "spent my lunchtime screening or doing other [research] stuff" (L. 934). ResN02 also reported previous charge nurses rostering her for afternoon or night shifts in the middle of the working week, making research difficult to do during the expected hours for the research role. ResN10 had encountered this problem just prior to the interview, and considered that 
this type of rostering indicated a lack of understanding by charge nurses about the requirements of the research role.

None of the PIs thought it was an absolute necessity for the ResNs to do clinical shifts, but it depended on the individual ResNs' level of clinical experience and familiarity to staff. PI06 stated that "even in the [research role], you're still doing some nursing" (L. 117). However, many of the PIs saw advantages to working clinical, including; enhancing communication and relationships $(n=3)$, raising the profile of research with a view to increasing recruitment into studies $(n=2)$, maintaining their skill level $(n=1)$ and understanding the work of the bedside nurse $(\mathrm{n}=1)$. This dual role was seen as advantageous by some PIs and ResNs who saw it as a way of getting greater value for the research department. "The plus for me is that obviously if [they're] on clinical then I can get some free research time out of them if we get a patient in a trial. So it increases our coverage a little bit" (PI01, L.97 99).

The NMs generally favoured the idea of ResNs doing clinical shifts $(n=5)$ although three thought it depended on the individual nurse and the length of time they had already worked clinically. Only two of these NMs were from ICUs where all of the ResNs did clinical shifts. NM04 ventured that having to do clinical was not important and was "an old notion" (L. 241). The reasons NMs gave for ResNs doing clinical were; to maintain their skill level $(n=3)$, to have credibility with patients, family and staff nurses $(n=3)$ and to have a presence on the unit $(\mathrm{n}=1)$. NM06 thought requiring ResNs to do clinical was an “idealistic, rather than practical expectation” (L. 242).

All participants were asked a benchmark question of what they would expect the ResNs to do if an emergency arose while they were with an ICU patient doing research duties. The examples given were that the patient had a cardiac arrest, or was trying to pull their endotracheal (breathing) tube out. All but one participant responded that the ResNs should intervene to their level of experience and ability. NM06 stated that as a registered nurse, that was their "duty of care". In contrast, NM05 stated that the ResNs should not get involved as it was no longer their area of expertise.

\section{Remuneration}

All of the ResNs were on a senior nurse salary scale of the Multi-Employer Collective Agreement (MECA) (DHB/NZNO, 2012). Of the seven who shared which level they were 
on, five were on Grade 2 and two were on Grade 3. Three ResNs volunteered that their research position was re-scoped between five and 10 years ago, which resulted in their pay scale not only being lowered but also their pay rate remaining the same for many years.

I was on level three and I went down to grade two and basically haven't had a pay rise for years, well not a proper one. So that was quite a sore point because I was regarded as the same level as an educator and suddenly it was a demotion, and that was quite a contentious time for me as well, by the then manager. (ResN06, L. 86 - 90)

There was discontent about the pay scale $(\operatorname{ResN} ; \mathrm{n}=7, \mathrm{PI} ; \mathrm{n}=2)$ and frustration that it was lower than ACNMs and educators despite similar levels of responsibility. "The salaries are on the nursing scale and we can't seem to get out of that" (PI04, L. 88).

The lack of a career structure was also a frustration and the research staff from one ICU has addressed this situation by introducing a career pathway. PI03 expressed the difficulty they experienced in securing this.

The organisation wanted everyone in research [in the hospital] on the same 'speciality clinical nurse research' position description and there was a big fight to get them to agree that that was inappropriate because you've got different people with different responsibilities and different experience. (L. $261-263)$

\section{After hours availability and pay}

There were very different expectations and remuneration for the nurses being 'on call' as seen in Table 11. Two ICUs had a formal, paid 'on call' system which meant they were expected to be available. In a further three ICUs, the ResNs were not paid to be on call, but were phoned if a patient needed to be recruited after the normal working day. The sixth ICU had no clinical trials running at the time of this study which required a ResN to be called in after hours.

One of the ResNs who was paid to be available commented that the on-call system was underutilised by ICU staff but conversely, if it was utilised more, their job would be even more demanding than it currently was. ResN09 said it was unclear how available she was expected to be, because she wasn't paid to be on-call and no formal system was in place. She commented that she was being called "more and more" and the expectation needed to be reviewed. If she was called in, she was paid at a higher call-back rate. NM01 thought 
the ResNs were expected to be available after hours to enrol patients if necessary, however the ResNs in this ICU stated that although they were usually available there was no on call roster and no obligation to come in. They were given 'time in lieu' rather than extra pay for being called back or working overtime, an arrangement made because of funding restrictions and FTE limitations. ResNs for another ICU were not paid to be on call and received extra pay at a normal rate if they came in to recruit a patient. Although not paid to be on call, ResNs from this ICU had experienced pressure from a PI to come and recruit patients after hours.

Table 11 On call and call back payments for ICU research nurses by ICU

\begin{tabular}{|l|l|l|l|l|l|l|}
\hline & \multicolumn{1}{|c|}{ Unit $*$} & \multicolumn{1}{|c|}{ Unit } & \multicolumn{1}{|c|}{ Unit } & \multicolumn{1}{|c|}{ Unit } & \multicolumn{1}{|c|}{ Unit } & \multicolumn{1}{|c|}{ Unit } \\
\hline On call times & Unsure & $0600-2200$ & $\begin{array}{l}\text { Only if } \\
\text { available }\end{array}$ & $\begin{array}{l}\text { At all } \\
\text { times }\end{array}$ & $\begin{array}{l}\text { Only if } \\
\text { available }\end{array}$ & $\begin{array}{l}\text { Not } \\
\text { required }\end{array}$ \\
\hline $\begin{array}{l}\text { On call } \\
\text { payment }\end{array}$ & Unpaid & MECA & Unpaid & MECA & Unpaid & $\begin{array}{l}\text { Not } \\
\text { required }\end{array}$ \\
\hline $\begin{array}{l}\text { Call back } \\
\text { remuneration }\end{array}$ & $\begin{array}{l}\text { MECA } \\
\text { Call back } \\
\text { rate }\end{array}$ & $\begin{array}{l}\text { MECA } \\
\text { Call back } \\
\text { rate }\end{array}$ & $\begin{array}{l}\text { Time in } \\
\text { lieu }\end{array}$ & $\begin{array}{l}\text { MECA } \\
\text { Call back } \\
\text { rate }\end{array}$ & $\begin{array}{l}\text { Normal } \\
\text { time }\end{array}$ & $\begin{array}{l}\text { Not } \\
\text { required }\end{array}$ \\
\hline
\end{tabular}

* ICU codes have not been included in this table to prevent the identifiability of ICUs and individual participants

Six of the ResNs volunteered they were available to be phoned after hours for advice. Many would also regularly phone the ICU to check that trial activities had been done correctly and on time. This out of hours work was recognised as important by the ResNs.

Fortunately I was rung at 11 o'clock at night and [gave advice] and I was very thankful to the doctors in that case ringing because as I have said many times a quick phone call would help solve issues or protocol violations. (ResN11, L. 638 - 642)

\section{Job descriptions and performance appraisals}

Seven ResNs said they had a job description, two were unsure and two said they didn't have one. Of the seven who had a job description, only three thought it was a true reflection of their role, and ResN11 stated that as she wrote hers, she was confident that it was correct. ResN06 had written hers many years prior when the role was quite different so although she was happy with the original job description, she no longer thought it was an accurate reflection of her role. In contrast, ResN02 stated that hers was a generic senior nurse job description, and not reflective of her research role and ResN09s included activities such as 
audit, which were not possible in the FTE she was employed for. ResN08 was in the process of having hers updated and ResN05 had never read her job description.

Performance appraisals were current in five of the ResNs, although one stated this was mainly for her nursing role. Two others had received a recent informal appraisal and ResN01, ResN09 and ResN11 had never had an appraisal for their research position. These ResNs had been in their research position between one and nine years, and two of them had a job description. ResN09 wondered how she would be appraised because "no one knows what you do" and "no one actually really knows what you would appraise" (L. 534, 537 538). ResN06 commented that she hadn't had a performance appraisal for many years because there had been several changes of manager during that time. Two ResNs who had had an up-to-date performance appraisal volunteered that it was done in consultation with staff they worked with including monitors, doctors and hospital research staff. This was called a "360 appraisal" and the ResNs thought it was done well.

All of the ICUs had more than one ResN working in a research role. Only one had a formal, tiered structure and career pathway for the ResN, with the senior nurse being called a research nurse coordinator. This was finalised around the time this study was conducted. In another, ResN11 said she was considered the senior in the department and delegated tasks to more junior ResNs. In addition, ResN09 reported that when staff worked in the department on secondments, they would be considered her junior and work would be delegated accordingly. The ResNs and PI in one ICU expressed a perception that the longer a ResN was in the role, the more senior they were, although this was not formal and all ResNs had the same title and pay. Two ResNs who had been in the role for a relatively short time regarded the experienced ResN as their senior, despite them being paid the same and sharing the same title. ResNs from three ICUs reported they were responsible for ICU staff who occasionally helped with research activities while they were on 'light duties' after an accident.

\section{Senior nurses}

ResNs were senior nurses as evidenced by their pay being on the senior pay scale of the MECA. However, many thought that the research role was not regarded as being a senior position within their ICU. Of the seven ResNs who saw themselves as seniors, three thought this was related to their ICU experience rather than being a ResN. The four who didn't think they were viewed by others as senior related this to the separateness of research 
from ICU. Of these four, three described themselves as "just the research nurse" and one as a "nurse with another job".

I think our role is that of a senior nurse but we're not involved in the operations of the unit so we wouldn't influence the development of the unit. It's a really bizarre role I think because you are a senior nurse but there's no senior nurses I think within this ICU apart from us that don't influence the day to day running of the unit. (ResN09, L. 659 - 663)

This uncertainty about where ResNs were placed in the nursing hierarchy was reinforced by NMs and was articulated by NM06 "It's separate, it's still got the job title, the role description, the responsibilities, however I don't believe staff at the bedside considers them their senior" (NM06, L. 148 - 149). This ambiguity was expressed by NM05 as:

Senior nurses here we would call charge nurses and the senior nursing team and then staff nurses, the senior staff nurses are all our level fours and very experienced and we've got a good core of them. I think that they [ResNs] would be seen from the floor as somewhere in-between. I don't think they're seen as part of the senior nursing team of the department because it's like that's more a management team. (L. 72 - 77)

These views were in contrast to the PIs, five of whom definitely thought that ResNs were senior nurses because of their speciality research and ICU knowledge. The sixth PI did not think they would be seen as senior nurses because of the separateness of research from the ICU.

\section{Clinical Trials Group meeting attendance}

The vast majority of PIs and NMs $(n=11)$ agreed that attending the main CTG in Australia was an essential part of the role and it was almost guaranteed that at least one ResN would attend. Attendance was considered important because of networking opportunities $(n=7)$, to learn about and participate in discussion about studies $(n=6)$ and find out study results $(n$ =2). Networking was regarded as important as other ResNs and RCs could serve as role models, and share experiences about specific trials, funding, and engendering a "research culture”. Knowing about studies enabled ResNs to participate in local discussions about which studies an ICU could participate in and enhanced the ResNs work. Both NM05 and PI01 commented that the nurses always came back "enthusiastic", and this was an element 
essential to involving other staff in research in the ICU. In contrast, one NM regarded attendance as a privilege that should be used as a reward for the ResNs doing their job well. The significant cost of attending research meetings overseas was acknowledged, and most were funded from the ICU research trust funds. Others applied to education funds within their hospitals to assist with paying for attendance and some ResNs had personal education fund allowances. Some ResNs attended other CTG meetings; however these were generally rated as less important than the main one.

The 10 ResNs who regularly attended the CTG meeting thought it was beneficial, that it assisted them to do their job well and was "part of the job". They identified similar reasons to the PIs and NMs including the opportunity to network with their Australian and NZ colleagues $(n=5)$, learning about upcoming trials $(n=6)$ and getting the results of trials they had contributed to $(n=2)$. Two ResNs did not like having to travel to Australia for the meetings and ResN09 stated that the usefulness of the CTG meeting was variable depending on the content. Two other ResNs regarded the RC Workshop as a good professional development opportunity, ResN09 stating there were few such opportunities for ResNs.

Without that [main CTG meeting] I wouldn't still be in the role... it reaffirms why I'm doing it ... I learn so much from it and its great knowing you've got a group of colleagues and that you are part of something that matters. If I was just here and not going to [CTG meeting] - just working here on my own, then I may as well just be entering data. (ResN10, L. 818 - 827)

\section{Summary}

This chapter has described the three groups of participants. Detailed information about the ICU research departments has helped to contextualise the structure the ResNs work within, and given a sense of how the role is perceived by those they work with. The ResNs demographics, background, qualifications and contractual working conditions have shown that the ResNs are experienced in ICU and well-qualified. Elements of dissatisfaction emerged around pay structures, job security and line management.

The next chapter presents the thematic findings drawn from the interviews and focuses on the work the ResNs do, to fulfil their role expectations. 


\section{Chapter 6 Findings: Research nurse role}

\section{Introduction}

This second findings chapter is about the actual work of the ResNs. There were three distinct areas which emerged from the thematic analysis about what was important about the role. The first is about the role of the ResN as project manager and reports their role in every aspect of trial management. Secondly, is the ResN role as a patient advocate, which focuses on the balance between patient and trial advocacy, and the ResN role in the consent process. The third area is about ResNs bridging knowledge gaps through their dual specialty and communication skills. This theme also identified skills and attributes important to being an ICU ResN. While job satisfaction was not a primary focus of this thesis, challenges experienced by the ResNs did emerge from the interviews and are presented in this section. Parts of their role were straight-forward such as the tasks that need to be performed for the safe conduct of clinical trials, while others such as communication and patient advocacy were more complex. Incorporated throughout this chapter are summary findings from the responsibility assignment matrix (RAM) which shows where there is consistency amongst participant groups and amongst units. When talking about the RAM, 'accountability' refers to the person who has the overall responsibility for a task, while 'responsibility' refers to the person who does the task. There was generally consensus about who did tasks within individual ICUs, however, RAM did highlight areas that participant groups within the same ICU didn't agree on a specific task. Areas where there was not agreement are highlighted in the text.

\section{Research nurses as project managers}

A responsibility which emerged over and over was that the ResN was a project manager or 'coordinator'. This role covered the different stages of managing clinical trials including design, selection, set up, maintenance, or day-to-day management, and trial closure. ResNs had involvement at every stage of the process and when ResNs didn't do the task themselves, they often facilitated it. The extent of their role within each stage varied depending on the skills required, how experienced they were and the complexity of the study. Some of the tasks were shared between the ResN and PI.

There are two main areas. One is in the initiation of a study, so there is a whole lot of setup type stuff and depending on skill sets the PI could do more or less or the nurse could do more or less but there are all of the ethics 
issues, all the paperwork that goes on with that, the contractual side of things and the reporting requirements that go along with that, both to the sponsor and to ethics and so on, so there's all that stuff that goes on in the setup and the background while the study is running and I think that's one area that's quite important and the other one is the actual operation of the study, where there is the screening and the support of recruitment consent and supporting whatever's required in terms of intervention in the bed space. (PI04, L.60 68)

Most of the ResNs described their last day working in research as 'typical'. It was extremely busy with many interruptions and they had to multi-task several activities simultaneously. There were similarities amongst the descriptions and all of the nurses had a basic daily routine which typically commenced with screening patients for trials, checking on patients who were already in studies and obtaining or facilitating consents. They then did a variety of other study duties, depending on what was outstanding and "dealing with what's thrown at you" (ResN09, L. 316).

An atypical day was mostly described by ResNs as one where they were either very busy or very quiet. If the ICU was really busy, many patients would be eligible for studies, including difficult studies which took all day to enrol one patient into. Other hallmarks of an atypical day included where study treatments didn't get administered correctly (protocol violations) and SAEs. An atypical day was also one where they "had no patients [in trials]" (ResN01, L. 331) or had "no interruptions and sitting in the office and able to do a full eight hours work, minus your coffee breaks” (ResN04, L 555 - 557).

It was widely acknowledged that PIs didn't have time to do most of the work for clinical trials, because of their clinical commitments as intensivists, and most of them weren't funded for research. PIs generally had a good understanding of what was involved to manage clinical trials within the ICU, and there was a sense of the nurses working alongside the PIs to manage the studies, however the day-to-day management of studies fell predominantly to the ResNs. The role had not been delegated in that PIs did not direct the work ResNs did daily, rather the ResNs took responsibility for their own work priorities. 
The ResNs in five ICUs were responsible for some part of the research finances, including maintaining budgets, writing invoices for patient payments or facilitating this process. Administration work included checking emails, looking for medical records, updating study folders, archiving, computer training and invoicing.

\section{Study duties}

\section{Trial design}

Five ResNs reported that they were currently a member of a management committee or had been in the past, which meant they had direct input into the trial design and protocol development. Additionally, two ResNs stated that they had been invited to be part of a CTG reviewing committee which meant they reviewed and had input into trial documents before being released to the wider research community. All participants thought it was important for ResNs to be on management committees and involved at the design stage because they had a good understanding about the practicalities of conducting the research, and impact on patients and nursing staff. Additionally, improving case report form (CRF) development and data dictionaries made their and other ResNs daily work easier. All ResNs working in ICU had the opportunity to review and contribute to CTG-endorsed studies, by way of attending CTG meetings. The ResNs reported much of their workload was for CTGendorsed studies.

\section{Trial selection}

Trial selection refers to deciding which trials an ICU participates in and has been included because ResNs are actively involved in this decision (Figure 7). When a study was being considered, the protocol was generally disseminated and discussed within the research team (ResNs and PI) before being sent to the other intensivists or a unit-level research committee for discussion. Scientific validity, equipoise, practicality, financial viability, clinical relevance and interest were taken into account when a study was being considered. Although the other specialists in the ICU were generally consulted, the interviews revealed that this was often a limited consultation. In Unit B, the ResN and PI decided together, and then the other intensivists were informed of the studies that were going to be done. A common finding for this part of the work was that both the PI and ResN were 'accountable' for particular tasks. Tasks such as feasibility data including number of patients likely to be recruited and the budget were collated by either the ResNs or PI, or done together which accounts for this finding. 
Figure 7 Responsibility assignment matrix - selecting studies for ICU

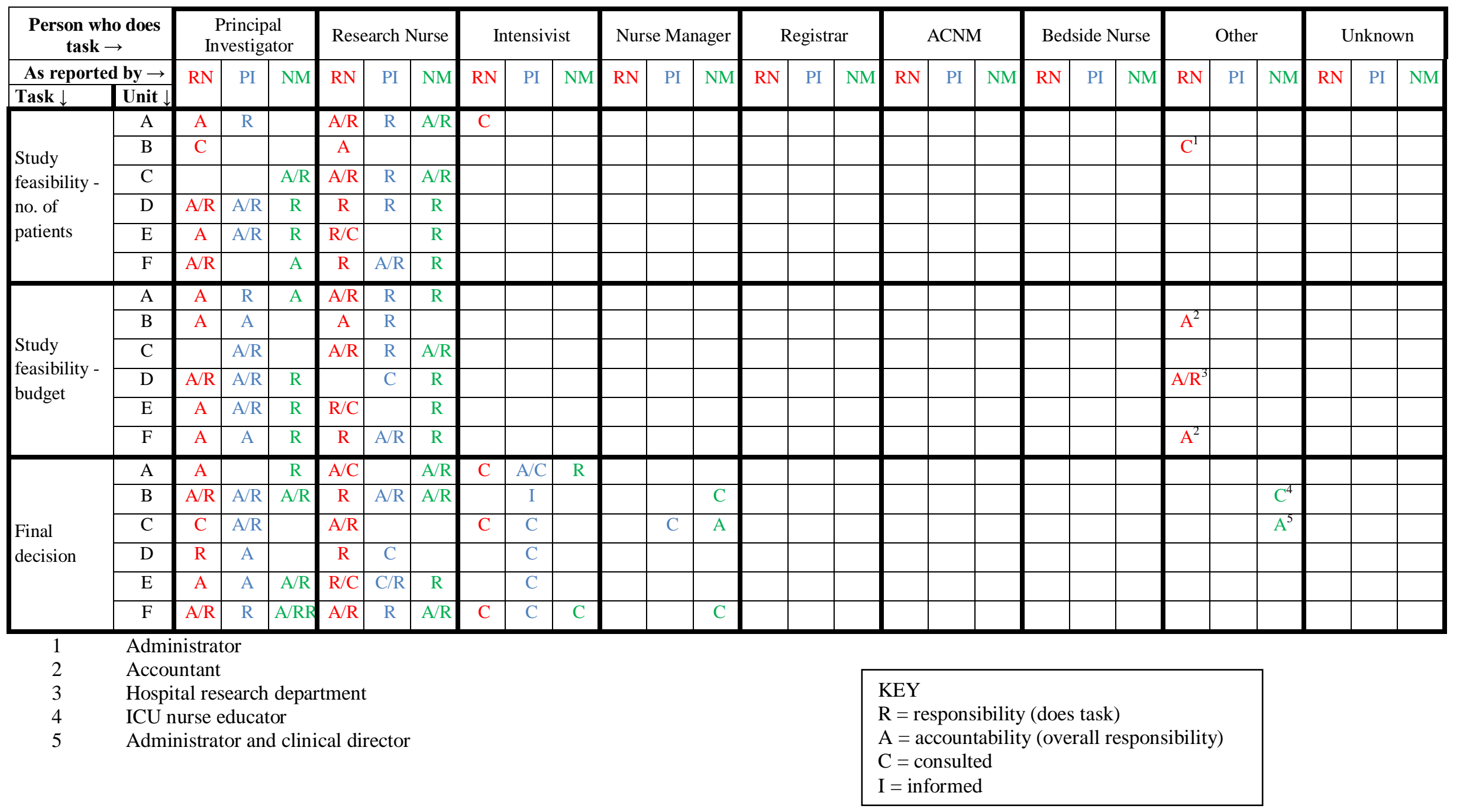


Few of the ResNs or PIs indicated that the NM was involved in this decision although two NMs stated they were consulted. In Unit C, the NM reported that the research account trustees who were the NM, clinical director and administrator made the final decision about which studies would be done. This was not however the view of the ResNs or PI in this ICU, who believed it was a research department decision, in consultation with the other intensivists and NM.

\section{Trial set up}

Once a trial had been accepted into ICU, documentation required for ethical review was typically the responsibility of the ResNs while the PI had ultimate accountability. "Then the research coordinator starts work. Everyone else forgets about it” (ResN10, L. 863). In five ICUs, one ResN would take ownership for the set up phase of a particular trial to ensure that everything was done, while in the sixth this responsibility fell solely to the most experienced ResN. Some of the ethics tasks were done by a ResN and PI together, or by the ResN alone. The exception to this was Maori consultation. The PIs $(n=5)$ clearly thought that ResNs had sole responsibility for this task, although four ResNs thought the PI had ultimate accountability for Maori consultation. The NMs reported that ResNs did these tasks for setting up trials, but were not always sure who had ultimate accountability.

The ResNs usually wrote the full ethics application, either on their own, or in conjunction with the PI. This included writing the information sheets and consent forms or adapting templates sent from the sponsor to make them more relevant to NZ. Five ResNs recorded that they had overall accountability for this task, while the remaining six stated that the PI had ultimate accountability. The PI would then read it, make any necessary adjustments and sign it. The locality assessment was usually obtained by the ResNs or facilitated by them. One ResN reported that she got the PI to do it because they had better access to the hospital level management who needed to approve the study being conducted in ICU.

The findings were similar for the documents required by the local hospital research department. The budget was generally written by a ResN or sometimes by the hospital accountant. Overall accountability for the budget lay either with the PI, the hospital research department and/or a hospital accountant. The ResN was responsible for organising and facilitating this process. In Unit E, the PI and NM thought the PI had overall accountability, while the ResNs, who facilitated the process, thought the hospital accountant had final sign-off. It was unclear in Unit $\mathrm{C}$ who had overall accountability for the budget. 
The ResNs thought that they and the accountant did the budget but didn't know who had overall accountability, the PI didn't know who did and the NM thought the ResNs did. Registering the research study with the hospital research department generally fell to the ResN, although in three ICUs, the PI had to sign the submission. In some cases, there wasn't a clear process and in one hospital, there was no research department.

There were differences in who was involved in contract negotiation between the sponsor and ICU. Commonly the ResNs would facilitate the process by consulting with the hospital legal department, with various roles within the hospital having ultimate sign-off. Five of the PIs stated they had overall accountability for the contract and in other cases only the legal department or hospital research department could do this. As can be seen in Figure 8, the NMs $(n=6)$ and most PIs $(n=5)$ were unaware that ResNs consulted the hospital lawyer or legal department regarding the trial contract in five ICUs.

Studies often required inter-departmental input and many ResNs talked about liaising with other departments to seek their support prior to the study commencing, in particular the emergency department (ED), pharmacy, laboratory and radiology (Figure 9). The hospital pharmacy was sometimes unable to support research due to resourcing limitations in which case, the ResNs facilitated suitable alternative staff to be the 'unblinded team' to store and dispense a study drug. This included recovery room staff or non-clinical nurses from within ICU. The ResNs liaised with ED about recruitment and management for particular studies. ResNs from four ICUs reported consulting the laboratory for quotes for local or study bloods and one ResN reported liaising with the radiology department for a particular study. Figure 9 shows relationships which exist in the setup and maintenance phases.

The final step for trial set up was getting the study into practice in the ICU, which involved educating doctors and nurses, and creating resources for staff. ResNs reported creating resource folders, posters, newsletters or updating information 'boards' about new trials which put the study duties into a more understandable form than the study protocol. Educating staff was identified as an ongoing process which continued throughout the study, however initial education was considered part of the trial set up. There was almost universal acceptance by all participant groups that the ResNs educated nurses about studies. The PIs were generally accountable for educating the doctors although the ResNs reported assisting with this. A common quote was "doctors for doctors, nurses for nurses". In 
Figure 8 Responsibility assignment matrix - trial set up stage (selected tasks)

\begin{tabular}{|c|c|c|c|c|c|c|c|c|c|c|c|c|c|c|c|c|c|c|c|c|c|c|c|c|c|c|c|c|}
\hline \multirow{2}{*}{\multicolumn{2}{|c|}{$\begin{array}{c}\text { Person who does task } \\
\rightarrow \\
\text { As reported by } \rightarrow \\
\end{array}$}} & \multicolumn{3}{|c|}{$\begin{array}{c}\text { Principal } \\
\text { Investigator }\end{array}$} & \multicolumn{3}{|c|}{ Research Nurse } & \multicolumn{3}{|c|}{ Intensivist } & \multicolumn{3}{|c|}{ Nurse Manager } & \multicolumn{3}{|c|}{ Registrar } & \multicolumn{3}{|c|}{ ACNM } & \multicolumn{3}{|c|}{ Bedside Nurse } & \multicolumn{3}{|c|}{ Other } & \multicolumn{3}{|c|}{ Unknown } \\
\hline & & RN & PI & NM & $\mathrm{RN}$ & PI & NM & $\mathrm{RN}$ & PI & NM & $\mathrm{RN}$ & PI & NM & RN & PI & NM & RN & PI & NM & $\mathrm{RN}$ & PI & NM & $\mathrm{RN}$ & PI & $\mathrm{NM}$ & $\mathrm{RN}$ & PI & NM \\
\hline \multirow{6}{*}{ Budget } & $\mathrm{A}$ & $\mathrm{A}$ & A & A & $\mathrm{A} / \mathrm{R}$ & $\mathrm{R}$ & $\mathrm{R}$ & & & & I & & & & & & & & & & & & $\mathrm{C}^{1}$ & & & & & \\
\hline & $\mathrm{B}$ & $\mathrm{A}$ & $\mathrm{A}$ & & $\mathrm{R}$ & $\mathrm{R}$ & $\mathrm{A} / \mathrm{R}$ & & & & & & & & & & & & & & & & & & $\mathrm{R}^{2}$ & & & \\
\hline & $\mathrm{C}$ & & & & $\mathrm{R}$ & & $\mathrm{A} / \mathrm{R}$ & & & & & & & & & & & & & & & & $\mathrm{R}^{1}$ & & & $\mathrm{~A}$ & $\mathrm{~A}$ & \\
\hline & $\mathrm{D}$ & & & & $\mathrm{R}$ & $\mathrm{C}$ & $\mathrm{A} / \mathrm{R}$ & & & & & & & & & & & & & & & & $\mathrm{A}^{3}$ & & & & & \\
\hline & $\mathrm{E}$ & & $\mathrm{A} / \mathrm{R}$ & $\mathrm{A} / \mathrm{R}$ & $\mathrm{R}$ & $\mathrm{C}$ & & & $\mathrm{C}$ & & & $\mathrm{C}$ & & & & & & & & & & & $\mathrm{A}^{1}$ & $\mathrm{C}^{1}$ & & & & \\
\hline & $\mathrm{F}$ & $\mathrm{A}$ & A & & $\mathrm{R}$ & $\mathrm{R}$ & $\mathrm{R}$ & & & & & & & & & & & & & & & & & & & & & \\
\hline \multirow{6}{*}{$\begin{array}{l}\text { Ethics } \\
\text { application }\end{array}$} & $\bar{A}$ & $\mathrm{~A} / \mathrm{R}$ & $\bar{A}$ & $\overline{\mathrm{A}}$ & $\mathrm{A} / \mathrm{R}$ & $\overline{\mathrm{R}}$ & $\mathrm{R}$ & & & & $\mathrm{I}$ & & & & & & & & & & & & & & & & & \\
\hline & B & & $\mathrm{A}$ & $\mathrm{R}$ & $\mathrm{R}$ & $\mathrm{R}$ & $\mathrm{R}$ & & & & & & & & & & & & & & & & & & & & & \\
\hline & $\mathrm{C}$ & & $\mathrm{A} / \mathrm{R}$ & & $\mathrm{A} / \mathrm{R}$ & $\mathrm{R}$ & $\mathrm{R}$ & & & & & & & & & & & & & & & & & & & & & \\
\hline & $\mathrm{D}$ & $\mathrm{A}$ & $\mathrm{A} / \mathrm{R}$ & $\mathrm{R}$ & $\mathrm{R}$ & $\mathrm{R}$ & $\mathrm{R}$ & & & & & & & & & & & & & & & & & & & & & \\
\hline & $\mathrm{E}$ & A & A & & $\mathrm{R}$ & $\mathrm{R}$ & & & & & & & & & & & & & & & & & & & & & & \\
\hline & $\mathrm{F}$ & A & A & & $\mathrm{R}$ & $\mathrm{R}$ & $\mathrm{R}$ & & & & & & & & & & & & & & & & & & & & & \\
\hline \multirow{6}{*}{$\begin{array}{l}\text { Contract } \\
\text { negotiation }\end{array}$} & $\mathrm{A}$ & $\mathrm{A} / \mathrm{R}$ & $\mathrm{A}$ & & $\mathrm{R}^{4}$ & $\bar{R}$ & $\mathrm{R}$ & & & & & & & & & & & & & & & & $A^{5}$ & & & & & \\
\hline & B & & $\mathrm{A}$ & & $\mathrm{A}$ & $\mathrm{R}$ & & & & & & & & & & & & & & & & & $\mathrm{C}^{5}$ & & & & & \\
\hline & $\mathrm{C}$ & A & $\mathrm{A} / \mathrm{R}$ & $\mathrm{C}$ & $\mathrm{A}$ & & $\mathrm{C}$ & & & & & & C & & & & & & & & & & $\mathrm{C}^{5}$ & & & & & \\
\hline & $\mathrm{D}$ & & C & $\mathrm{A} / \mathrm{R}$ & & C & & & & & & & & & & & & & & & & & $\mathrm{A}^{3}$ & $\mathrm{~A}^{3}$ & & & & \\
\hline & $\mathrm{E}$ & & $\mathrm{A} / \mathrm{R}$ & $\mathrm{R}$ & & & & & & & & & I & & & & & & & & & & $\mathrm{A} / \mathrm{C}^{5}$ & & & & & $\mathrm{~A}$ \\
\hline & $\mathrm{F}$ & A & $\mathrm{A} / \mathrm{R}$ & $\mathrm{A} / \mathrm{R}$ & $\mathrm{R}$ & $\mathrm{R}$ & & & & & & & & & & & & & & & & & & & & & & \\
\hline \multirow{6}{*}{$\begin{array}{l}\text { Implementing } \\
\text { the protocol } \\
\text { ('makes it } \\
\text { happen') }\end{array}$} & $\mathrm{A}$ & & & & $\mathrm{A} / \mathrm{R}$ & & $\mathrm{A} / \mathrm{R}$ & & & & & & & & & & & & & & & & & & & & & \\
\hline & B & & & & $\mathrm{A} / \mathrm{R}$ & & & & & & & & & & & & & & & & & & & & & & & \\
\hline & C & & $\mathrm{R}$ & & $\mathrm{A} / \mathrm{R}$ & $\mathrm{R}$ & $\mathrm{A} / \mathrm{R}$ & & & & & & & & & & & & & & & & & & & & & \\
\hline & $\mathrm{D}$ & & $\mathrm{R}$ & & $\mathrm{A} / \mathrm{R}$ & $\mathrm{A} / \mathrm{R}$ & $\mathrm{A} / \mathrm{R}$ & & & & & & & & & & & & & & & & & & & & & \\
\hline & $\mathrm{E}$ & & $\mathrm{R}$ & & $\mathrm{A} / \mathrm{R}$ & $\mathrm{R}$ & $\bar{R}$ & & $\mathrm{R}$ & & & & & & & & & & & & & & & & & & & \\
\hline & $\mathrm{F}$ & & & & $\mathrm{A} / \mathrm{R}$ & $\mathrm{A} / \mathrm{R}$ & $\mathrm{A} / \mathrm{R}$ & & & & & & & & & & & & & & & & & & & & & \\
\hline \multicolumn{29}{|c|}{$\begin{array}{ll}1 & \text { Accountant } \\
2 & \text { Finance department } \\
3 & \text { Hospital research department } \\
4 & \text { Facilitates } \\
5 & \text { Hospital lawyer or legal department }\end{array}$} \\
\hline
\end{tabular}


contrast, the ResN in Unit B was responsible for all research related education for doctors and nurses.

\section{Figure 9 Key functional relationships}

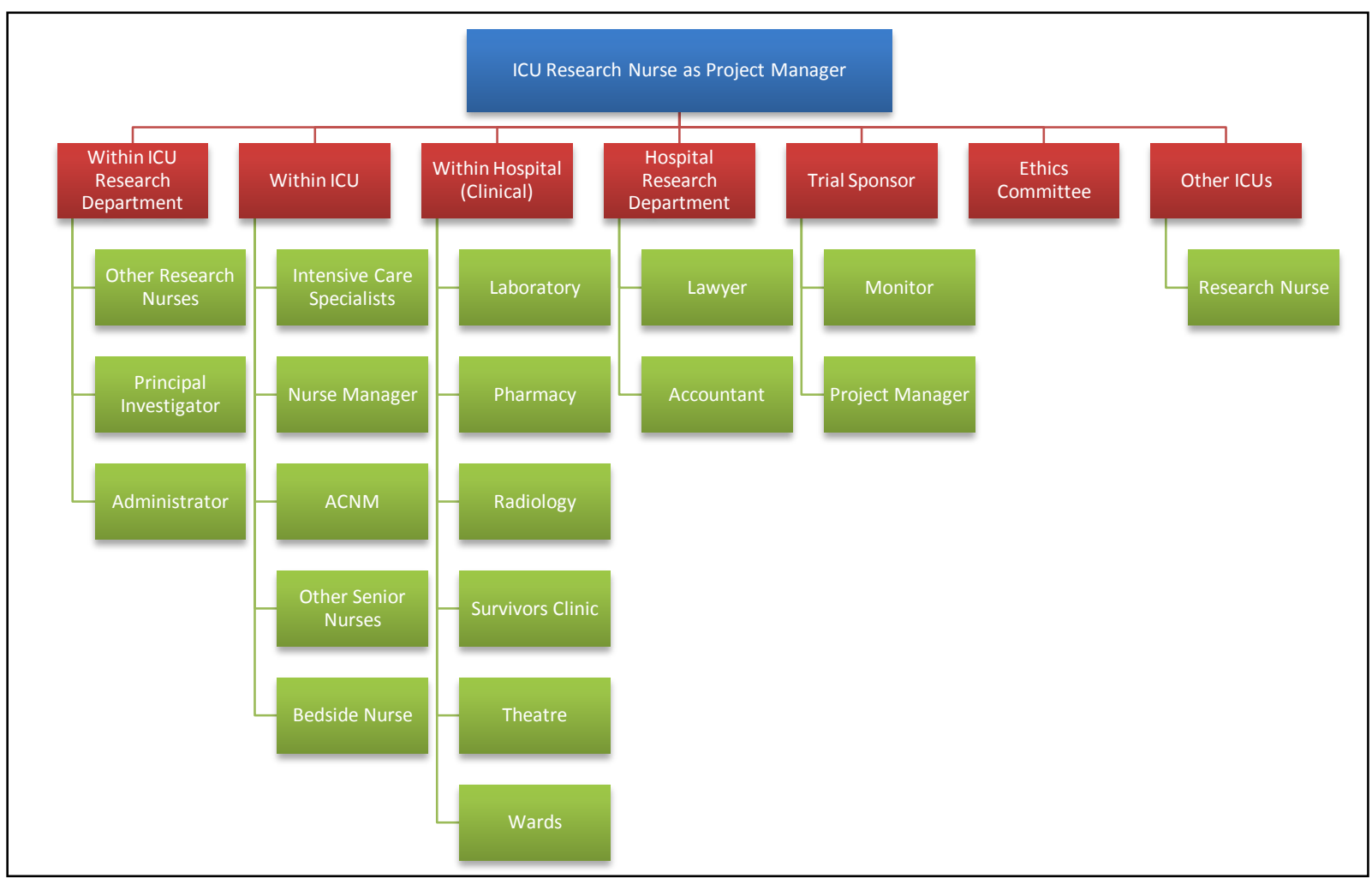

\section{Trial maintenance}

The majority of the ResNs' time was spent managing the various aspects of clinical trials. This was their 'daily work'. ResNs within research departments communicated by way of communication books, whiteboards and email, about which patients were already in studies, what issues were outstanding and if there were patients who could potentially be enrolled. Within each department everyone was responsible for all study maintenance tasks, including recruiting, consenting and data collection so ResNs had to know about all of the trials their ICU was participating in (Chapter 5, Table 9).

They'd be responsible for basically following the protocol which is encouraging, educating the unit staff, following the protocol, encouraging and enrolments, trying to avoid breaches of the protocol and making sure there's a paper trail of our activities for audit and follow up and trying to make sure those things are available for when we do get audited by the larger groups. (PI02, L. 105 - 111) 


\section{Screening and recruitment}

Screening and recruiting patients into trials was a very large part of trial management. There was variability about who identified patients eligible for trials. Among these were the ResN, PI, other intensivists, registrars, ACNMs and the bedside nurses. While most participants indicated when the RAM questions were asked that all of these groups were expected to recruit, the interviews revealed that the majority of patients were identified and recruited by the ResNs and PIs. This practice was more prevalent in some ICUs than others. In Unit F, almost all patients were identified by either the ResNs or PI although patients could only be enrolled into a study if the intensivist on call for the day agreed to it. A common reason given for screening and recruitment being the research team's responsibility was that nurses were too busy caring for acutely unwell patients.

The calibre of patients has changed so much in terms of the acuity. They've [clinical staff] just got too much else going on, and then to think about 'does this patient actually qualify for any studies'? We try and get the word out there but at the end of the day it's quite difficult if you have got a really sick patient and you're trying to get your headspace around $X, Y, Z$ and inotropes and whatever else. It's not $A$ [airway], $B$ [breathing], $C$ [circulation], Research. (ResN05, L. 440 - 446)

ResNs screened patients for eligibility for clinical trials early in the day and then throughout the day as new patients were admitted to the ICU. One ICU worked closely with the ED on a study which was commenced in the ED but was run by ICU research staff. This meant the ResNs screened in ED as well as ICU for patients.

The ResNs maintained the screening log which documented patients who were considered for studies but found to be ineligible or eligible but not enrolled ('missed'). When eligible patients were missed, there was variability about whether the PI or ResN was responsible for following up with staff. In some ICUs this was not a major consideration, the ResNs were philosophical about 'missing' patients and no feedback was given to staff. In others, every patient admitted was considered as being eligible for a study, unless they met an exclusion criterion, and staff were followed up about every missed patient by the ResNs or PI. Ensuring that consents had been obtained for patients in trials was part of the ResNs' daily routine and is discussed at length in the section about patient advocacy. 


\section{Protocol adherence}

Several of the ResNs talked about how they ensured a study protocol was adhered to. This entailed the ResNs doing study activities themselves and checking to see that delegated study tasks had been performed by bedside nurses, doctors or other staff. These delegated tasks included ensuring patients received the correct trial treatment at the correct time. Monitoring the patient involved ensuring that specific vital observations and blood tests were taken at the correct time. In four ICUs, the ResNs centrifuged blood tests and stored them in the research freezer themselves; while in the remaining two ICUs, ResNs ensured the blood tests were taken and sent to the laboratory for processing. If study interventions or monitoring occurred during the night or weekend, the ResNs organised it, delegating tasks to ACNMs or intensivists, and then checked by telephone to ensure that it happened correctly. All who did this stated that they were still responsible for the study interventions occurring correctly and in the correct time-frame and some volunteered that they preferred to come in after hours and do it themselves, rather than delegate it. There was a degree of anxiety about having to delegate some study tasks and various methods were employed to ensure these tasks were done correctly including education, pre-labelling blood tubes, filling in laboratory forms in advance, highlighting when tasks were due on the ICU flow chart, phoning staff to check, sending texts to intensivists, creating trial-specific instructions with tick boxes and checking blood results remotely from home. ResN11 verbalised the stress when she had to delegate study tasks such as taking and processing study bloods to other personnel when she wasn't there. She constantly checked that the delegated tasks had been done within the correct timeframes. Part of trial maintenance also included on-going checking of study supplies including study fluid and laboratory supplies and checking the research fridges and freezers were working correctly.

\section{Recognising protocol violations and serious adverse events}

The vast majority of protocol violations were recognised by the ResNs, although ResN08 stated that "occasionally" an ACNM might notice something had been done incorrectly and report it to the ResN. Protocol violations were either noticed at the time they occurred, when ResNs checked on patients throughout the day, or later when data were being collected. NM04 reported that bedside nurses would also realise and self-report, however this was not corroborated by the ResNs or PI in their ICU. ResN09 shared that it was reasonable for ResNs to notice protocol violations as they were the ones who knew the protocol in depth. Participants in some ICUs reported that protocol violations were rare, 
and the number of protocol violations varied depending on the studies ICUs were participating in.

Reporting and documenting of protocol violations was primarily the role of the ResNs, however this was often done in conjunction with the PI. The PIs had ultimate accountability for the violations and ResNs stated that the PI was informed if a violation was noticed, and in most cases, signed it off. In Unit B, all protocol violations were registered as 'reportable events' because the patient had not received the correct treatment. No similar organisational reporting was done in any other hospital.

Serious adverse events (SAEs) were uncommon but time-consuming when they occurred. The SAE reporting documentation was usually written by the ResNs although there was consensus that the PI had ultimate accountability for signing these off. SAEs involved investigation of what had happened and ResN02 reported that when they happened, they took "a big chunk of the day", gathering and collating the information to report to the sponsor and the lead site.

\section{Data collection and data entry}

Ten of the ResNs talked about data collection and data entry. In most cases this was done on a daily basis, although ResN04 preferred to do it all at once after the patient had left ICU. The PIs rated data collection as a key role that ResNs did, and for PI02, it was the most important thing they did. ResNs regarded high quality data collection as important because it respected the patient's contribution to the study and ensured that the study findings were valid. However, three ResNs expressed that the role was "more than data collection".

Outcome data were generally collected by phoning the patient, and enquiring after their well-being. An assessment was also made during this phone call about whether any adverse events had occurred since the patient was in ICU or hospital. ResNs primarily conducted the follow-up phone calls or if they were unable to contact the patient, gathered this information from the patient's family, computer system, general practitioner (GP) or outpatient clinics. ResNs reported that when contacted, patients frequently sought advice about non-research related issues that were concerning them. The ResNs generally referred patients to the appropriate health-care provider or GP to address these matters. In some cases, they were able to offer explanations if it was related to their treatment in ICU. 
ResN11 reported that in the past, phoning patients had been delegated to other staff, but now preferred to do it herself so that she knew of any issue or difficulty reaching the patient.

\section{Trial closure}

The final step in any study is disseminating results when they are published. Disseminating results to the nursing staff was seen as the responsibility of the ResN and was done by way of 'communication books', newsletters, education sessions and posting results on research notice boards. This was described by NM01 as "clos[ing] the loops to find out, has it actually made a difference" (L. 336 - 337). In addition to informing staff of the results, ResNs also sent results to study participants who had requested them during the consent process.

\section{Research nurses balancing patient needs with research requirements}

The ResNs contributed to balancing the rights and needs of the patient and their family, with the demands of the research at every stage of the trial process. This theme is about their role as patient advocates throughout the study and in particular, during the consent process.

It starts right from the beginning, making sure you're putting the right patient into the study so that the study gets the right participants and is a true reflection of that group. So you're protecting the study requirements and then you're making sure that the intervention that's to be delivered is delivered correctly. And you might see that at the bedside or you might see it when you're collecting the data and realise that there's been a mistake made or something's gone wrong and then you're educating the staff at the same time in terms of the study requirements, what to watch out for, for adverse events or whatever. So you need some knowledge of the patient and the care that's being provided whether it's a standard care or the intervention. And then educating the family when you're talking to them for consent and the same with the patient hopefully later on as well. So making sure that the patient is getting the best possible care, that they're not at risk by being in the study. And that the study data and study requirements are all met and true. (ResN08, L. 738 - 752)

\section{Patient advocacy}

There were different interpretations of patient advocacy amongst the ResNs. ResN06 stated that the term 'patient advocacy' was interchangeable with patient safety, and she and 
ResN02 said this was the main role of the ResN. Some ResNs advocated for patients by offering them the opportunity to participate in research, while others reported advocacy as refraining from offering some patients this opportunity. Others thought patient advocacy was present right from when the protocol was written. Protocol adherence was also seen as patient advocacy as it ensured the patient was kept safe and the study results were trustworthy. ResN06 stated "we are basically, in a lot of cases we are the only people standing between another National Women's and they don't recognise that. They don't recognise the risk to the organisation if things aren't done properly" (L. 1204 - 1208). 'National Womens' refers to a landmark case in NZ which much of our current ethics legislation is based on $(\mathrm{MOH}, 2007)$. This viewpoint was more extreme than beliefs expressed by most ResNs.

ResNs talked of using their clinical judgement to refrain from enrolling patients into clinical trials, who they assessed as unsuitable, despite their meeting the inclusion criteria. This was sometimes done without the knowledge of the PI. ResN06 and ResN07 had both acted in a patient's interests by not offering them studies. In one example, this involved supporting a family who wanted a patient to be enrolled into a study but thought the patient would not want to participate. ResN06 encouraged them to make the decision based on what the patient would have wanted, not what the family wanted and the patient was not enrolled. In another example, ResN07 had screened a patient for a study which required insertion of a central venous line (CVL). The patient was frail and elderly and would not have needed the CVL for their usual care, so ResN07 did not suggest the study. She expressed doubt that this was the way a ResN should behave "because I know that as a research nurse we should be going for it but I just think sometimes oh what are we doing, what are we doing?" (L. 479 - 480). ResN02 stated that making a clinical judgement about the suitability of a patient being offered a trial, above and beyond the inclusion and exclusion criteria was definitely the role of the ResN.

One example is choosing not to put patients in a study or even approach it with them because even though they fit the protocol, they may have been diagnosed with terminal $\mathrm{Ca}$ [cancer] or they've got terminal Ca or you look at them, they'll say yes, but it's going to put an undue pressure on them to do things, and onerous things where they have to do this or that, and they're just not up to it, but they would do it. (L. 800 - 806) 
ResN01 shared a clinical situation when she was a patient advocate in her role as a ResN. Throughout the course of a study intervention for a patient on the ward, she assessed that the patient was sicker than initially thought, and she facilitated their admission to ICU.

Three ResNs said that most of the time, advocacy was ensuring patients and their families were given the opportunity to be in a study. ResN11 described this as a "patient's right" and ResN08 reported advocacy went both ways but more frequently involved arguing for patients to go into studies than being kept out. The remaining ResNs answered in general terms about patient advocacy. They didn't have examples but volunteered they definitely thought it was part of their role and would intervene if necessary to ensure patients' interests were upheld. Although ResN04 and ResN09 thought it was done so automatically that they didn't think about it, they both talked about patients in special circumstances e.g. patients with an intellectual disability or with dementia potentially being enrolled under the delayed consent option, when they could never actually consent for themselves. ResN09 stated that ResNs in the ICU setting tended to advocate more for the relatives than the patient themselves.

Three of the ResNs volunteered that patient advocacy began at the trial design stage, thus validating the need for ResNs to be involved in writing the protocol and be on trial management committees. ResN02 talked about ensuring that the protocol was written in a way that the research question would be answered and the patients would be kept safe, and then adhered to so that a patient's contribution was worthwhile. Ensuring the protocol was adhered to and the data collected was of a high quality was seen as a way of respecting the patient's contribution.

Two ResNs and one PI (ResN02, ResN04, PI05) made references to patients doing better overall when patients were involved in a research trial because care was delivered correctly and on time. ResN11 also pointed out that patient care such as blood results were scrutinised much more carefully if a patient was in a trial.

\section{Consent process}

The part of the ResNs role with the greatest diversity amongst ICUs, was the issue of consent. There were different levels of responsibility for obtaining consent, depending on the nature of the study and potential risk and side effects of study treatment. The very minimum ResNs did in the process of consenting patients into clinical trials was to identify 
potential patients and if they were eligible, give them or their family an information sheet about the trial. If the family seemed interested, they would facilitate a doctor to obtain consent. At the other end of the scale, one ResN was able to complete the full consent process for all clinical trials, including a commercial trial for an experimental drug. The difference amongst practices tended to be at the ICU or hospital policy level, combined with the personal view of the individual ResN. The PI and ResNs for two ICUs reported that the majority of consents were obtained by the research team.

To make it easier to understand, the subject of consent is split into lower-risk studies which compare two standard existing treatments and where delayed consent is an option, and higher-risk studies in which prior assent or consent must be obtained (usually from a surrogate) prior to the commencement of any study procedures. In reality, the degree of risk in research studies is on a continuum, rather than dichotomous, as there is always the possibility of side effects because the result of any treatment is unknown when it is being researched. The scale of 'Always, Usually, Sometimes, Rarely, Never' has been applied based on the findings from the interviews (Table 12). For this table, the view of the ResN has been given more weight, as the assumption has been made that they know whether they obtain consents or not. As will be seen in the RAM charts, the NMs were not always aware or certain of the level of involvement ResNs had in the consent process. This could be because of a recent change in practice or the NM just not knowing.

\section{Lower risk}

Examples of lower risk were a study about glucose control in ICU patients (NICE Study Investigators, 2009) and comparison between two intravenous fluids for fluid resuscitation (CHEST Trial) (The CHEST Management Committee, 2011). For these lower risk studies the ResNs in five of the ICUs were able to obtain prior or delayed written consent for specific trials from the patient (Figure 10). The ResN from one of these ICUs did not obtain assents from family because of her interpretation of the wording in the Health and Disability Code of Rights: Right 7, which states that where a patient is not competent, health service delivery is 'in the patient's best interests' (Health and Disability Commisioner, 2009). A ResN in this ICU shared that this was a decision only a doctor could make. She also highlighted the issue of indemnity, and questioned who would be responsible if something went wrong with a trial if it was the ResN who had obtained assent. In all five of the ICUs where the ResNs were able to obtain written consent, they were almost exclusively the staff who obtained follow-up consent from the patient once they were competent, when 
the initial assent had been signed by a family member. ResNs from two ICUs stated that they had only recently started obtaining consents and it was much easier than having to wait for doctors to do it.

Table 12 Research nurses' role in the consent process

\begin{tabular}{|l|l|l|l|l|l|l|l|}
\hline \multicolumn{1}{|c|}{ UNIT } & \multicolumn{1}{|c|}{ A } & \multicolumn{1}{c|}{ B } & \multicolumn{1}{c|}{ C } & \multicolumn{1}{c|}{ D } & \multicolumn{1}{c|}{ F } \\
\hline Lower Risk Study & Usually & Sometimes & Sometimes & Usually & Usually & Usually \\
\hline $\begin{array}{l}\text { Discuss study } \\
\text { (Patient or Family) }\end{array}$ & Usually & Sometimes & Sometimes & Never & Sometimes & Usually \\
\hline $\begin{array}{l}\text { Patient consent } \\
\text { (prior) }\end{array}$ & Usually & Sometimes & Sometimes & Never & Sometimes & Usually \\
\hline $\begin{array}{l}\text { Patient consent } \\
\text { (delayed) }\end{array}$ & Usually & Sometimes & Sometimes & Never & Never & Usually \\
\hline $\begin{array}{l}\text { Relative assent } \\
\text { Follow up consent } \\
\text { (patient) }\end{array}$ & Usually & Usually & Usually & Never & Usually & Usually \\
\hline $\begin{array}{l}\text { Higher Risk Study } \\
\text { Discuss study } \\
\text { (Patient or Family) }\end{array}$ & Rarely & Sometimes & Sometimes & Usually & Usually & Usually \\
\hline $\begin{array}{l}\text { Patient consent } \\
\text { Relative assent }\end{array}$ & Never & Never & Never & Never & Never & Never & N/A \\
\hline $\begin{array}{l}\text { Follow up consent } \\
\text { (patient) }\end{array}$ & Never & Never & Never & Never & Never & N/A \\
\hline
\end{tabular}

a dependent on seniority and of the research nurse and whether they are a sub-investigator

b experimental study had pre-hospital consent only

In Unit D, where ResNs never obtained written consents or assents, this was the policy of the hospital research department. Although these Unit D ResNs were not able to obtain written consent, they were involved in the consent process. It was their role to screen the patient for eligibility and they usually spoke to the family (or patient), gave them the information sheet to read and answered any questions they had. After this the PI would answer any additional questions and get the consent form signed.

In some instances, although obtaining consent was part of the ResN's role they used their judgement with particular patients to decide whether it would be more appropriate for a doctor to obtain written consent. ResN03 and ResN08 said they gauged potential family reaction to research and also tried to minimise the impact or distress to families by 
Figure 10 Responsibility Assignment Matrix - consent process (lower risk studies)

\begin{tabular}{|c|c|c|c|c|c|c|c|c|c|c|c|c|c|c|c|c|c|c|c|c|c|c|c|c|c|c|c|c|}
\hline \multirow{2}{*}{\multicolumn{2}{|c|}{$\begin{array}{c}\begin{array}{c}\text { Person who does } \\
\text { task } \rightarrow\end{array} \\
\text { As reported by } \rightarrow\end{array}$}} & \multicolumn{3}{|c|}{$\begin{array}{l}\text { Principal } \\
\text { Investigator }\end{array}$} & \multicolumn{3}{|c|}{ Research Nurse } & \multicolumn{3}{|c|}{ Intensivist } & \multicolumn{3}{|c|}{ Nurse Manager } & \multicolumn{3}{|c|}{ Registrar } & \multicolumn{3}{|c|}{ ACNM } & \multicolumn{3}{|c|}{ Bedside Nurse } & \multicolumn{3}{|c|}{ Other } & \multicolumn{3}{|c|}{ Unknown } \\
\hline & & & & & & & & & & & & & & & & & & & & & & & & & & & & \\
\hline Task $\downarrow$ & Unit & RN & PI & NM & $\mathrm{RN}$ & PI & NM & $\mathrm{RN}$ & PI & $\mathrm{NM}$ & RN & PI & $\mathrm{NM}$ & $\mathrm{RN}$ & PI & $\mathrm{NM}$ & RN & PI & NM & RN & PI & NM & $\mathrm{RN}$ & PI & NM & $\mathrm{RN}$ & PI & NM \\
\hline \multirow{6}{*}{$\begin{array}{l}\text { Discusses } \\
\text { study with } \\
\text { patient or } \\
\text { family }\end{array}$} & $\mathrm{A}$ & $\mathrm{R}$ & & & $\mathrm{A} / \mathrm{R}$ & $\mathrm{A} / \mathrm{R}$ & $\mathrm{A} / \mathrm{R}$ & $\mathrm{R}$ & & & & & & I & & & & & & I & & & & & & & & \\
\hline & B & $\mathrm{R}$ & & & $\mathrm{A} / \mathrm{R}$ & $\mathrm{R}$ & A/R & $\mathrm{R}$ & & & & & & $\mathrm{R}$ & & $\mathrm{R}$ & & & & & & & & & & & & \\
\hline & $\mathrm{C}$ & $\mathrm{A}$ & $\mathrm{R}$ & & $\mathrm{R}$ & $\mathrm{R}$ & $\mathrm{R}^{1}$ & $\mathrm{R}$ & $\mathrm{R}$ & $\mathrm{A}$ & & & & & & & & & & & & & & & & & & $\mathrm{A}$ \\
\hline & $\mathrm{D}$ & $\mathrm{A} / \mathrm{R}$ & $\mathrm{A} / \mathrm{R}$ & $\mathrm{R}$ & $\mathrm{R}$ & $\mathrm{R}$ & $\mathrm{R}$ & $\mathrm{A} / \mathrm{R}$ & & $\mathrm{R}$ & & & & & & & & & & & & & & & & & & \\
\hline & $\mathrm{E}$ & $\mathrm{R}$ & $\mathrm{R}$ & & $\mathrm{A} / \mathrm{R}$ & $\mathrm{R}$ & & $\mathrm{R}$ & & & & & & & & & & & & & & & & & & & & \\
\hline & $\mathrm{F}$ & $\mathrm{A} / \mathrm{R}$ & $\mathrm{A} / \mathrm{R}$ & & $\mathrm{R}$ & $\mathrm{A} / \mathrm{R}$ & $\mathrm{A} / \mathrm{R}$ & & $\mathrm{R}$ & & & & & & & & & & & & & & & & & & & \\
\hline \multirow{6}{*}{$\begin{array}{l}\text { Obtains } \\
\text { written } \\
\text { consent - } \\
\text { Patient }\end{array}$} & A & $\mathrm{A} / \mathrm{R}$ & & $\mathrm{R}$ & $\mathrm{A} / \mathrm{R}$ & $\mathrm{A} / \mathrm{R}$ & $\mathrm{A} / \mathrm{R}$ & $\mathrm{A} / \mathrm{R}$ & & $\mathrm{R}$ & & & & I & & $\mathrm{R}$ & & & & I & & & & & & & & \\
\hline & $\mathrm{B}$ & $\mathrm{A} / \mathrm{R}$ & $\mathrm{A} / \mathrm{R}$ & & $\mathrm{R}$ & $\mathrm{R}$ & $\mathrm{A} / \mathrm{R}$ & $\mathrm{R}$ & & & & & & $\mathrm{R}$ & $\mathrm{R}$ & $\mathrm{R}$ & & & & & & & & & & & & \\
\hline & $\mathrm{C}$ & $\mathrm{A}$ & $\mathrm{R}$ & & $\mathrm{R}$ & $\mathrm{R}$ & $\mathrm{R}^{1}$ & $\mathrm{R}$ & $\mathrm{R}$ & $\mathrm{A}$ & & & & & & & & & & & & & & & & & & \\
\hline & $\mathrm{D}$ & $\mathrm{A} / \mathrm{R}$ & $\mathrm{A} / \mathrm{R}$ & $\mathrm{R}$ & & & $\mathrm{R}$ & $\mathrm{A} / \mathrm{R}$ & & $\mathrm{R}$ & & & & & & & & & & & & & & & & & & \\
\hline & $\mathrm{E}$ & $\mathrm{A} / \mathrm{R}$ & $\mathrm{A} / \mathrm{R}$ & & $\mathrm{R}$ & & & $\mathrm{A} / \mathrm{R}$ & & & & & & & & & & & & & & & & & & & & \\
\hline & $\mathrm{F}$ & $\mathrm{A} / \mathrm{R}$ & $\mathrm{A} / \mathrm{R}$ & & $\mathrm{R}$ & $\mathrm{A} / \mathrm{R}$ & $\mathrm{A} / \mathrm{R}$ & & $\mathrm{I}$ & & & & & & & & & & & & & & & & & & & \\
\hline \multirow{6}{*}{$\begin{array}{l}\text { Obtains } \\
\text { written } \\
\text { assent - } \\
\text { Family* }\end{array}$} & A & $\mathrm{A} / \mathrm{R}$ & & $\mathrm{R}$ & $\mathrm{A} / \mathrm{R}$ & $\mathrm{A} / \mathrm{R}$ & $\mathrm{A} / \mathrm{R}$ & $\mathrm{A} / \mathrm{R}$ & & $\mathrm{R}$ & & & & I & & & & & & I & & & & & & & & \\
\hline & $\mathrm{B}$ & $\mathrm{A} / \mathrm{R}$ & $\mathrm{A} / \mathrm{R}$ & & $\mathrm{R}$ & $\mathrm{R}$ & $\mathrm{A} / \mathrm{R}$ & $\mathrm{R}$ & $\mathrm{R}$ & & & & & $\mathrm{R}$ & $\mathrm{R}$ & $\mathrm{R}$ & & & & & & & & & & & & \\
\hline & $\mathrm{C}$ & $\mathrm{A}$ & $\mathrm{R}$ & & $\mathrm{R}$ & $\mathrm{R}$ & $\mathrm{R}^{1}$ & $\mathrm{R}$ & $\mathrm{R}$ & $\mathrm{A}$ & & & & & & & & & & & & & & & & & & \\
\hline & $\mathrm{D}$ & $\mathrm{A} / \mathrm{R}$ & $\mathrm{A} / \mathrm{R}$ & $\mathrm{R}$ & & & $\mathrm{R}$ & $\mathrm{A} / \mathrm{R}$ & & $\mathrm{R}$ & & & & & & & & & & & & & & & & & & \\
\hline & $\mathrm{E}$ & $\mathrm{A} / \mathrm{R}$ & $\mathrm{A} / \mathrm{R}$ & & & & & $\mathrm{A} / \mathrm{R}$ & & & & & & & & & & & & & & & & & & & & \\
\hline & $\mathrm{F}$ & $\mathrm{A} / \mathrm{R}$ & $\mathrm{A} / \mathrm{R}$ & & $\mathrm{R}$ & $\mathrm{A} / \mathrm{R}$ & $\mathrm{A} / \mathrm{R}$ & & I & & & & & & & & & & & & & & & & & & & \\
\hline \multirow{6}{*}{$\begin{array}{l}\text { Obtains } \\
\text { written } \\
\text { follow-up } \\
\text { consent - } \\
\text { Patient }\end{array}$} & A & & & & $\mathrm{A} / \mathrm{R}$ & $\mathrm{A} / \mathrm{R}$ & $\mathrm{A} / \mathrm{R}$ & I & & & & & & & & & & & & I & & & & & & & & \\
\hline & B & & $\mathrm{A} / \mathrm{R}$ & & $\mathrm{R}$ & $\mathrm{R}$ & $\mathrm{A} / \mathrm{R}$ & & $\mathrm{R}$ & & & & & & & $\mathrm{R}$ & & & & & & & & & & & & \\
\hline & $\mathrm{C}$ & A & & $\mathrm{R}$ & $\mathrm{R}$ & $\mathrm{R}$ & $\mathrm{R}^{1}$ & $\mathrm{R}$ & $\mathrm{R}$ & & & & & & & & & & & & & & & & & & & $\mathrm{A}$ \\
\hline & $\mathrm{D}$ & $\mathrm{A} / \mathrm{R}$ & $\mathrm{A} / \mathrm{R}$ & $\mathrm{R}$ & & & $\mathrm{R}$ & $\mathrm{A} / \mathrm{R}$ & & $\mathrm{R}$ & & & & & & & & & & & & & & & & & & \\
\hline & $E$ & & & & $\mathrm{~A} / \mathrm{R}$ & & & & & & & & & & & & & & & & & & & & & & & \\
\hline & $\mathrm{F}$ & $\mathrm{A} / \mathrm{R}$ & $\mathrm{A} / \mathrm{R}$ & & $\mathrm{R}$ & $\mathrm{A} / \mathrm{R}$ & A/R & & & & & & & & & & & & & & & & & & & & & \\
\hline
\end{tabular}

1 Facilitates

*Refers to Relative/Whanau member/Friend assent

$\mathrm{A}=$ accountability (overall responsibility)

$\mathrm{C}=$ consulted

$\mathrm{I}=$ informed 
combining clinical trial discussions with family meetings about a patient's general condition.

\section{Higher risk studies}

Higher risk studies generally involved an experimental therapy, such as an unlicensed drug, new treatment or procedure, or a drug where one purpose of the study was to evaluate the risks of potentially harmful side effects compared to the beneficial use of the drug. They were often, but not always commercial studies. These studies tended to be for the sickest ICU patients who had a higher likelihood of poor outcomes including death.

In most ICUs, the RAM results indicated that only an intensivist, who was also a subinvestigator for a study could obtain consent/assent from a patient or family for these higher risk studies (Figure 11). The role of the ResN in these situations was often to approach the family about the possibility of a trial and gauge the likelihood of them agreeing to a study. The ResNs were sometimes present in the family meeting about the trial, along with the intensivist and assisted with answering questions about the research. ResN11 also talked about going over the information sheet with a sub-investigator before they spoke to the family, and sitting in the meeting to ensure the information was correct, as she was more familiar with the protocol. Reasons given for ResNs not consenting for higher risk studies were that "doctors could explain complicated procedures better" (ResN02, L. 1244) and a belief that a "doctor should consent for novel and early phase studies" (ResN11, L. 303).

\section{Delayed consent}

Delayed consent had been used for studies in all of the participants' ICU. The ResNs and PIs had a good understanding of delayed consent, while half of NMs either weren't familiar or had limited understanding of it. Amongst those participants who were familiar with it, there was universal support for the process of delayed consent for ICU patients participating in clinical trials. Most ResNs and PIs considered research would be impossible in the ICU setting without it. However, all were conscious that such consents did not get misused or inappropriately used and several talked about using it "judiciously", and stating that every practicable effort was made to contact family prior to enrolling a patient into a clinical trial. 
Figure 11 Responsibility Assignment Matrix - consent process (higher risk studies)

\begin{tabular}{|c|c|c|c|c|c|c|c|c|c|c|c|c|c|c|c|c|c|c|c|c|c|c|c|c|c|c|c|c|}
\hline \multirow{2}{*}{\multicolumn{2}{|c|}{$\begin{array}{c}\begin{array}{c}\text { Person who does } \\
\text { task } \rightarrow\end{array} \\
\text { As reported by } \rightarrow\end{array}$}} & \multicolumn{3}{|c|}{$\begin{array}{c}\text { Principal } \\
\text { Investigator }\end{array}$} & \multicolumn{3}{|c|}{ Research Nurse } & \multicolumn{3}{|c|}{ Intensivist } & \multicolumn{3}{|c|}{ Nurse Manager } & \multicolumn{3}{|c|}{ Registrar } & \multicolumn{3}{|c|}{ ACNM } & \multicolumn{3}{|c|}{ Bedside Nurse } & \multicolumn{3}{|c|}{ Other } & \multicolumn{3}{|c|}{ Unknown } \\
\hline & & & & & & & & & & & & & & & & & & & & & & & & & & & & \\
\hline Task $\downarrow$ & Unit & $\mathrm{RN}$ & PI & NM & $\mathrm{RN}$ & PI & $\mathrm{NM}$ & RN & PI & $\mathrm{NM}$ & $\mathrm{RN}$ & PI & NM & $\mathrm{RN}$ & PI & $\mathrm{NM}$ & $\mathrm{RN}$ & PI & NM & $\mathrm{RN}$ & PI & NM & $\mathrm{RN}$ & PI & $\mathrm{NM}$ & $\mathrm{RN}$ & PI & NM \\
\hline \multirow{6}{*}{$\begin{array}{l}\text { Discusses } \\
\text { study with } \\
\text { patient or } \\
\text { family }\end{array}$} & $\mathrm{A}$ & $\mathrm{A} / \mathrm{R}$ & $\mathrm{A} / \mathrm{R}$ & & $\mathrm{R}$ & & $\mathrm{A} / \mathrm{R}$ & $\mathrm{A} / \mathrm{R}$ & $\mathrm{A} / \mathrm{R}$ & & & & & $\mathrm{I}$ & & & & & & I & & & & & & & & $\mathrm{A}$ \\
\hline & B & $\mathrm{A} / \mathrm{R}$ & & & $\mathrm{C}$ & $\mathrm{R}$ & & $\mathrm{A} / \mathrm{R}$ & & & & & & & & & & & & & & & & & & & & \\
\hline & $\mathrm{C}$ & $\mathrm{A} / \mathrm{R}$ & $\mathrm{A} / \mathrm{R}$ & $\mathrm{C}$ & & & $\mathrm{R}^{1}$ & $\mathrm{~A} / \mathrm{R}$ & $\mathrm{A} / \mathrm{R}$ & $\mathrm{A}$ & & & & & & & & & & & & & & & & & & \\
\hline & $\mathrm{D}$ & $\mathrm{A} / \mathrm{R}$ & $\mathrm{A} / \mathrm{R}$ & $\mathrm{R}$ & $\mathrm{R}$ & $\mathrm{R}$ & $\bar{R}$ & $\mathrm{~A} / \mathrm{R}$ & & $\mathrm{R}$ & & & & & & & & & & & & & & & & & & A \\
\hline & $\mathrm{E}$ & $\mathrm{A} / \mathrm{R}$ & $\mathrm{A} / \mathrm{R}$ & & $\mathrm{R}$ & & & $\mathrm{R}$ & & & & & & & & & & & & & & & & & & & & \\
\hline & $\mathrm{F}$ & $\mathrm{A} / \mathrm{R}$ & $\mathrm{A} / \mathrm{R}$ & & $\mathrm{R}$ & $\mathrm{A} / \mathrm{R}$ & $\mathrm{A} / \mathrm{R}$ & $\mathrm{R}$ & & & & & & & & & & & & & & & & & & & & $\mathrm{A}$ \\
\hline \multirow{6}{*}{$\begin{array}{l}\text { Obtains* } \\
\text { written } \\
\text { consent - } \\
\text { Patient }\end{array}$} & $\mathrm{A}$ & $\mathrm{A} / \mathrm{R}$ & $\mathrm{A} / \mathrm{R}$ & $\mathrm{R}$ & & & & $\mathrm{A} / \mathrm{R}$ & $\mathrm{A} / \mathrm{R}$ & $\mathrm{R}$ & & & $\mathrm{R}$ & I & & & & & & I & & & & & & & & \\
\hline & B & $\mathrm{A} / \mathrm{R}$ & $\mathrm{A} / \mathrm{R}$ & & & & & $\mathrm{A} / \mathrm{R}$ & $\mathrm{A} / \mathrm{R}$ & & & & & & & & & & & & & & & & & & & $\mathrm{A}$ \\
\hline & C & $\mathrm{A} / \mathrm{R}$ & $\mathrm{A} / \mathrm{R}$ & $\mathrm{C}$ & & & $\mathrm{R}^{1}$ & $\mathrm{~A} / \mathrm{R}$ & & $\mathrm{A}$ & & & & & & & & & & & & & & & & & & A \\
\hline & D & $\mathrm{A} / \mathrm{R}$ & $\mathrm{A} / \mathrm{R}$ & $\mathrm{R}$ & & & $\mathrm{R}$ & $\mathrm{A} / \mathrm{R}$ & & & & & & & & & & & & & & & & & & & & \\
\hline & $\mathrm{E}$ & $\mathrm{A} / \mathrm{R}$ & $\mathrm{A} / \mathrm{R}$ & & & & & $\mathrm{A} / \mathrm{R}$ & & & & & & & & & & & & & & & & & & & & \\
\hline & $\mathrm{F}$ & $\mathrm{A} / \mathrm{R}$ & $\mathrm{A} / \mathrm{R}$ & & $\mathrm{A} / \mathrm{R}^{2}$ & $\mathrm{~A} / \mathrm{R}$ & $\mathrm{A} / \mathrm{R}$ & & I & & & & & & & & & & & & & & & & & & & A \\
\hline \multirow{6}{*}{$\begin{array}{l}\text { Obtains } \\
\text { written } \\
\text { assent - } \\
\text { Family** }\end{array}$} & A & $\mathrm{A} / \mathrm{R}$ & $\mathrm{A} / \mathrm{R}$ & $\mathrm{R}$ & & & & $\mathrm{R}$ & $\mathrm{A} / \mathrm{R}$ & $\mathrm{R}$ & & & $\mathrm{R}$ & I & & & & & & I & & & & & & & & \\
\hline & B & $\mathrm{A} / \mathrm{R}$ & $\mathrm{A} / \mathrm{R}$ & & & & & $\mathrm{A} / \mathrm{R}$ & $\mathrm{A} / \mathrm{R}$ & & & & & & & & & & & & & & & & & & & $\mathrm{A}$ \\
\hline & $\mathrm{C}$ & $\mathrm{A} / \mathrm{R}$ & $\mathrm{A} / \mathrm{R}$ & $\mathrm{C}$ & & & $\mathrm{R}^{1}$ & $\mathrm{~A} / \mathrm{R}$ & $\mathrm{A} / \mathrm{R}$ & $\mathrm{A}$ & & & & & & & & & & & & & & & & & & $\mathrm{A}$ \\
\hline & D & $\mathrm{A} / \mathrm{R}$ & $\mathrm{A} / \mathrm{R}$ & $\mathrm{R}$ & & & $\mathrm{R}$ & $\mathrm{A} / \mathrm{R}$ & & $\mathrm{R}$ & & & & & & & & & & & & & & & & & & \\
\hline & $\mathrm{E}$ & $\mathrm{A} / \mathrm{R}$ & $\mathrm{A} / \mathrm{R}$ & & & & & $\mathrm{A} / \mathrm{R}$ & & & & & & & & & & & & & & & & & & & & \\
\hline & $\mathrm{F}$ & $\mathrm{A} / \mathrm{R}$ & $\mathrm{A} / \mathrm{R}$ & & $\mathrm{A} / \mathrm{R}^{2}$ & $\mathrm{~A} / \mathrm{R}$ & $\mathrm{A} / \mathrm{R}$ & & & & & & & & & & & & & & & & & & & & & A \\
\hline \multirow{6}{*}{$\begin{array}{l}\text { Obtains } \\
\text { written } \\
\text { follow-up } \\
\text { consent - } \\
\text { Patient }\end{array}$} & A & $\mathrm{A} / \mathrm{R}$ & $\mathrm{A} / \mathrm{R}$ & & $\mathrm{R}$ & & $\mathrm{A} / \mathrm{R}$ & $\mathrm{A} / \mathrm{R}$ & $\mathrm{A} / \mathrm{R}$ & & & & & & & & & & & & & & & & & & & \\
\hline & B & $\mathrm{A} / \mathrm{R}$ & $\mathrm{A} / \mathrm{R}$ & & & & & $\mathrm{A} / \mathrm{R}$ & $\mathrm{A} / \mathrm{R}$ & & & & & & & & & & & & & & & & & & & A \\
\hline & C & $\mathrm{A} / \mathrm{R}$ & $\mathrm{A} / \mathrm{R}$ & $\mathrm{C}$ & & & $\mathrm{R}^{1}$ & $\mathrm{~A} / \mathrm{R}$ & $\mathrm{A} / \mathrm{R}$ & $\mathrm{A}$ & & & & & & & & & & & & & & & & & & $\mathrm{A}$ \\
\hline & D & $\mathrm{A} / \mathrm{R}$ & $\mathrm{A} / \mathrm{R}$ & $\mathrm{R}$ & & & $\mathrm{R}$ & $\mathrm{A} / \mathrm{R} / \mathrm{C}$ & & $\mathrm{R}$ & & & & & & & & & & & & & & & & & & \\
\hline & $\mathrm{E}$ & $\mathrm{A} / \mathrm{R}$ & & & $\mathrm{R}$ & & & & & & & & & & & & & & & & & & & & & & & \\
\hline & $\mathrm{F}$ & $\mathrm{A} / \mathrm{R}$ & $\mathrm{A} / \mathrm{R}$ & & $\mathrm{A} / \mathrm{R}^{2}$ & $\mathrm{~A} / \mathrm{R}$ & $\mathrm{A} / \mathrm{R}$ & & & & & & & & & & & & & & & & & & & & & \\
\hline \multicolumn{29}{|c|}{ 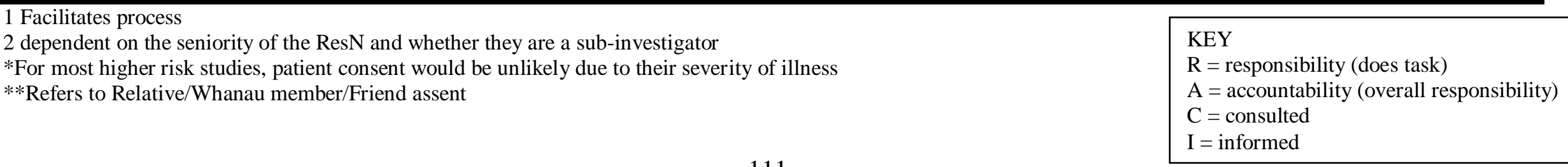 } \\
\hline
\end{tabular}


Reasons given for supporting delayed consent as an option for ICU studies included pragmatic reasons such as initiating study treatment in a timely fashion by being able to put a patient who was unconscious and without family present into a study and ethical reasons such as giving families more time to consider a study. ResN08 questioned whether approaching families or patients for assent or consent in ICU could be seen as "informed", even if it was obtained prior to initiation of study treatment. This was because families were often upset and unable to comprehend the information being given to them, and that delayed consent as an option meant they could consider the study in their own time.

Some participants (ResN, $\mathrm{n}=5$; PI, $\mathrm{n}=1$ ) volunteered that delayed consent was only appropriate to use in low risk studies. Two examples of using delayed consent for patients who were conscious were given. In both cases, the study had been discussed with the patient, and they had agreed to participate, but research staff assessed that the patient was unable to make an informed decision due to their illness (ResN01, ResN07).

\section{Difficulties with consent}

A difficulty highlighted with delayed consent was when and by whom the patient or family should be approached about the study and consent or assent sought. Most participants agreed that it was best to inform the patient or family that the patient was in a clinical trial as soon as practically possible. However, the ResNs and PI in one ICU strongly thought that it should be a member of the research team who approached the patient or family. They stated that from past experience it was more important to wait for a member of the research team and if it was sought sooner by the bedside nurse, consent was more likely to be refused.

So we would see the family as soon as we feasibly can at an appropriate timewhen they're not too distressed, and just let them know who we are, and that they're in the study. If it's appropriate you could leave them the information sheet at that point, well I'd go back the next day and catch up with them and introduce myself again, and say this is what we're doing, "are you happy?", give them the information sheet and go back at an appropriate time. You just have to play it by ear, all the families are different, but try and get the family consent as soon as possible - certainly let them know about the study as soon as you can. And then when the patient's better, you obviously follow up the patient on the ward and get the patient's consent. (ResN03, L. 300 - 308) 
In contrast, ResN06 stated that the patient or family should be told immediately by whichever staff member saw them first (usually the bedside nurse). PI01 stated that withdrawal of consent after a patient being enrolled into a study using the delayed consent option was very low. ResN09 spoke about missing consents after a patient was enrolled in a study using the delayed consent option. If ResNs worked part-time, it was possible for the patient to have been discharged to the ward or home by the time the ResN became aware that they were even in the study. This scenario made on-going consent "difficult to get” (ResN09, L. 1011).

An additional difficulty in ICU was identifying the most appropriate person to talk to about research and ICU staff relied on family members identifying themselves as having a close relationship to the patient (ResN04). It was also common to have to talk to several people and need them all to agree. This was echoed by ResN11, who shared a situation where consent was obtained from one family member, then another, closer relative arrived, and the consent process was gone through again.

Two ResNs had concerns about the consent process when doctors, who were not specifically involved in research, obtained consent from patients or their families. ResN09 and ResN11 were uncertain that these doctors always had sufficient knowledge to obtain consents. ResN09 questioned how "informed" the consents were and ResN11 reported feeling anxious about how correctly consents were done when some doctors did them. The correct procedure, with correct dates, times and patients filling in all relevant sections were all cause for anxiety.

\section{Research nurses bridging gaps and making research happen}

This theme is about the necessity of having a ResN dedicated to research in ICU. It also addresses key attributes including whether they need to be an ICU nurse. The reasons cited as important for being an ICU nurse are grouped into specialist knowledge, communication, relationships and 'other' attributes, which are discussed below. The first three of these are interrelated and are about the importance of being an ICU nurse. This section also discusses barriers to performing the job well.

\section{Essential to research}

Most of the contribution to patient care was described as long-term, and that the ResN was integral to the process of researching to find "best practice" (NM04). ResNs were seen as essential for enabling ICUs to participate in a research programme. Their dual role as 
specialists in research and ICU was seen as advantageous for bridging knowledge gaps for patients and nurses, in particular. Having the knowledge and ability to combine the two specialties in a way that kept the patient safe and produced high quality research was recognised as an important part of the ResN role. There was universal agreement from PIs that without ResNs there would be little or no research in ICU. The ResNs were described variously as the "link" (NM04), "lynchpin" (PI04), "driver of research" (NM04) and the "glue that makes research happen" (PI01). Some reasons for these descriptions were pragmatic and included that the ResNs provided continuity by being dedicated to the research role and that they had time which PIs just didn't have.

They make it happen. It would not happen. I mean we tried to do a little bit of stuff here back in the early 90s with nobody and it was just about impossible really. I mean I, it's essential that we have them ... so not only do they bring anything its kind of the whole lynchpin of the whole system really is a good research coordinator. (PI04, L. 590 - 593)

\section{Specialist knowledge}

Amongst the ResN participants, there was a continuum of answers about the necessity of ICU ResNs being an ICU nurse. These answers ranged from agreement being an ICU nurse would help with the job $(n=2)$ to strong opinion that ICU ResNs needed to be ICU nurses $(n=6)$, through to the need that they needed to be very senior ICU nurses $(n=3)$. ResN01 and ResN03 stated that although being an ICU nurse would help, it wasn't essential and probably depended on the individual. Two ResNs who had experience working with non-ICU and nonnurse researchers in ICU strongly believed that you needed to be an ICU nurse to be an ICU ResN, in order to understand the complexities of the ICU patient and environment.

Being proficient in ICU was generally considered more important than a background in research as it was considered that research could be taught more easily than ICU care. All participants from one ICU universally supported this notion because of past experience, where a ResN who was experienced in research, but not ICU, had been employed. This had been problematic and a conscious decision had been made for future employments to add more weight to ICU experience and ability to work with the research team.

The NMs cited many reasons that ICU experience would be advantageous including the need for clinical skills unique to nursing and ICU, relationships with bedside nurses (credibility), 
understanding the nursing perspective, severity of illness of ICU patients and ICU clinical data. NM04 and NM05 stated that it was important to be a senior or expert nurse and NM01 stated they should be at least a proficient-level, intensive care trained nurse. NM06 volunteered that in a larger research department, it may be appropriate to have some non-nurses.

All PIs agreed that that the "ideal" ICU ResN would be an ICU nurse and the role would be very difficult if the ResNs weren't ICU nurses first. PI06 however speculated that it would be possible to incorporate non-nurses into a larger research department, and PI02 thought it would be possible for someone with a non-nursing health background to work in the role of an ICU ResN. PI03 and PI06 volunteered that ResNs should have worked to quite a senior level or have 'significant' ICU experience. The reasons they gave were relationships with staff, knowledge of ICU for data collection and the ability to talk to patients about non-research clinical problems. PI06 highlighted that being more senior meant the ResNs had a "degree of trust and respect out there amongst your peers" (L. 97 - 98).

A reason given for needing to be an ICU nurse was the holistic view that nurses have and their ability to be able to bring the needs of the patient into research. This intertwining of roles and patient advocacy were discussed in the previous section. ICU trained nurses knew what was expected for the study and also assisting with the clinical components of the study. Several ResNs said if you employed a data collector, then that is all you would get. By knowing what was required for research and ICU care, the impact on the bedside nurse and therefore patient care could be minimised (ResN02, ResN06).

They need to be able to look at the patient in a very rounded view to see because it's not just about trial - it's about assessing the patient, having a disease prediction about where that disease could potentially go and if they're going to fill the trial or not, so we've made it a proficient qualified critical care nurse. NM01 (L. 178 - 182)

Three areas of clinical knowledge regarded as specific to ICU included ventilation, an area most ICU nurses would be experts on, interpretation of arterial blood gases (ABGs) and 'monitoring' patients. Monitoring in the ICU context refers to both the close monitoring of patients through high nurse/patient ratios, and cardiac monitoring. PI05 had recent experience with engineering $\mathrm{PhD}$ students who had little clinical understanding about ventilation which 
reinforced their belief that ICU ResNs should be ICU nurses. ResN01 also thought having ICU expertise was advantageous because you could do trial activities yourself, and not have to rely on the bedside nurses doing it. ResN07, ResN10 and PI03 stated that you could do the research job without being a nurse in other clinical areas, but definitely not in ICU.

\section{Communication and education}

Communication was reported as a very important skill by most of the ResNs $(n=10)$. This was articulated either as "communication", "good people skills" or "rapport". For ResN02 and 10 , there was a need for not only good verbal communication, but also written communication. ResNs were able to communicate with a wide sphere of people from stressed families, through to ICU nurses and medical specialists, and ethics committees. They had expertise in research, but because of their additional expertise in ICU were able to understand and apply complicated research protocols and make them more understandable for patients and nurses. ResNs also reported that being an ICU nurse helped to understand the clinical implications of a study from the bedside nurse's perspective.

Communication with patients and families was important to ensure they had a good understanding of the research trial. Having an ICU background was also important for communicating with patients and their families about all of their clinical issues (not just research). ResN06 stated that ResNs could "take some of the stress off nurses by talking to families" (L. 1049) and PI06 volunteered that as ICU nurses, the ResNs were "comfortable talking about research" (L. 469) to families and patients.

Communication with staff was essential to ensure the smooth-running of clinical trials. This began before the trial even started, with educating nurses and doctors about what the trial involved. When a patient was on a trial, the ResNs communicated with the bedside nurses, ACNMs, PI and other intensivists to ensure protocol adherence and the safety of the patient. ResNs delegating duties to bedside nurses has already been described in the first part of this section. In the ICU setting, ResNs had to have clinical knowledge in order to ensure the safe conduct the trials. Their extensive knowledge of individual study protocols meant they could 'translate' information for other nursing staff. Several ResNs volunteered that they talked to the bedside staff as part of their daily routine and educated them informally about research studies. 


\section{Relationships}

The NMs described the ResNs being the link between research and the bedside nursing staff. Many studies increased the workload of the bedside nurse, by requiring more laboratory testing, monitoring or delivery of a therapeutic substance. ResNs reported working closely with the bedside nurse to ensure that the research protocol was conducted safely and within given timeframes. To alleviate the increased workload of trials on the bedside nurse, ResNs often did clinical tasks which were required for the study, such as putting up intravenous or enteral feeds, taking routine blood tests when study bloods were taken and assisted with nursing cares. The two reasons for this were to ease the burden to the bedside nurse's workload and to ensure that it was done correctly and in a timely manner. It was widely reported by NMs that nurses were more likely to discuss aspects of the study with nurses, rather than an intensivist and ResN11, who had worked with medical and $\mathrm{PhD}$ students reported that nurses responded better to nurses than non-nurses. She felt that rapport with the nurses and the respect earned from being a senior ICU nurse also helped with research. ResN03 also thought that nurses responded better to nurses.

Maintaining relationships with bedside nurses was important for research, as without the input of bedside nurses, research was difficult. Part of this relationship was being seen as being clinically "credible" by other staff, and 'visible' in and around ICU. Being part of the team has already been discussed in Chapter 5. Two ResNs talked about wearing their nursing uniform or scrubs to feel like part of the team. Visibility was seen as important for recruitment and for staff relations. Ways of achieving visibility included having an office within ICU and being out on the unit.

\section{Other attributes}

The PIs stated that attributes such as "attention to detail", "enthusiasm" and "being organised" were very important. The ResNs identified additional attributes as "methodical", "computer and numeric skills", "good time management", "good detective work" and "diplomacy" as essential to the role. The ability to motivate the team and engender enthusiasm was identified frequently because engendering a research culture within an ICU was the biggest barrier to conducting research. This belief was shared by all participant groups and was expressed as "marketing" (NM02), "motivate the team" (PI01), "raise the profile", (NM02) and "enthusiasm" (ResN04). PI05 and NM06 also stated that a tertiary level degree, such as a Masters (or working towards one) was important. 


\section{Nursing research}

A vocal minority which included one ResN, one PI and two NMs from different ICUs thought the ResN role should include more focus on nursing research. Other ResNs $(n=8)$ reported that their role with regards to nursing and other primary research was more of an advisory and support role. This was mainly for staff undertaking their Masters degrees and most advice was regarding ethics. As stated earlier, two ResNs were conducting their own studies. Four of the NMs reported that ResNs assisted with nursing research in an advisory and support role, and that they had particular knowledge about the ethics programme. NM01 expressed frustration that the ResN role was solely clinical trials and not nursing research. NM06 reported that part of the role was to "promote and lead nursing research in the ICU” (L. 198). NM02 and NM04 stated there was no nursing research programme in their ICUs, and therefore the ResNs had no involvement in nursing research.

\section{Role challenges}

Although there was widespread satisfaction among ResNs, two main challenges were identified and echoed by the PIs and some NMs. These challenges which are interrelated were "lack of a research culture" and "staff obstructiveness", both of which are discussed below. These were identified as barriers to enrolling patients into trials, and were in no way related to patient safety.

\section{Research culture}

The issue of "lack of research culture" was reported by participants from four ICUs. They expressed frustration that research only happened when the ResNs or PIs were present to recruit patients and had few solutions about how to "get people who are not really about research to screen for you" (PI01, L. 603). One suggestion was employing ResNs with "enthusiasm" and "passion" for research. Research staff wanted to change the culture of the unit so that research was an integral part of ICU care, however as ResN04 articulated "Everybody believes in evidence based practice and they like to think that they do it, but there's a real aversion if it requires any extra work" (L. 719 - 721). For PI05

It's changing the culture so that everybody knows that they're part of this research thing and it's not just for the money and it's not because you're short [of money] but you're in intensive care and you should be doing research. (L. 472 - 474) 
This lack of research culture was certainly not unanimous and all participants in one ICU expressed a strong research culture which extended to senior doctors, registrars, charge nurses and bedside nurses.

\section{Staff}

The most common challenge identified by participants was a perception of "staff obstructiveness" or "disinterest". The obstructive staff most commonly referred to were (nonPI) intensivists, as well as some senior nursing staff, such as ACNMs. These difficulties with intensivists (other than the PI) were reported in four ICUs. This was proffered not only by the ResNs $(n=8)$, but also the PIs $(n=3)$ and NMs $(n=2)$. At the lower end of the spectrum, the ResNs knew that depending on which intensivist was in charge for the day, they would need to make a "good case" to be allowed to approach a patient for a study. At the other end of the spectrum NM05 stated that "one or two [senior] doctors here who are quite obstructive and always don't want the patients in the trials" (L. 610 - 611). In some cases, these intensivists had participated in the decision-making about whether units were going to participate in a study.

It [research] comes way down the list. And, and that's the feeling you getthat it's completely down the bottom of the list so it's the last thing anyone thinks of and us research nurses we have to just make it fit, they're not going to help us and it's all up to us to make it work. (ResN10, L. 555 - 558)

This was seen as a medical problem and ResN02 stated the solution needed to come from "the top". However, ResN08 reported there was a lack of support from all higher levels of nursing management, in particular the NM and ACNMs.

Not feeling as though you have the support from the nursing leadership of all levels and again I think it starts at the charge nurse level. I think there's a couple of charge nurses [ACNMs] who...I don't know if they intentionally set out to sabotage or just do it because they don't get it, but there could certainly be a lot more support after hours from that side. And from the [NM]. (L. 896 - 901)

Many ResNs volunteered that it depended which clinical staff were working and in particular, the ACNM, as to how well research worked. When ResN02 was talking about protocol adherence, she shared 
that's just part of the job, and part of the job is realising if you get a 100 people, 50\% actively try to help you do it, $30 \%$ passively try and help you do it, 5\% will do things wrong if it occurs to them, and the other 10-15\% will actively try and sabotage it without them putting it that way. So it helps to work in a unit where you know your staff, so you know which ones to keep an eye out for and which ones to say 'thank [goodness] that ones on'. (L. 918 924)

\section{Future development of the role}

Much of the discussion about the future development of the ResN role was about recognition and the development of a career pathway. This incorporated recognition of the work ResNs do, responsibility they have and their contribution to patient care and research $(n=6)$. Two ways of showing recognition were identified as the advent of a career pathway $(n=6)$ and an appropriate level of pay $(n=6)$. Additionally recognition needed to be through the Nursing Council of NZ $(n=1)$ and NZNO $(n=2)$ and nursing in general $(n=1)$. ResN02 was "cynical" about the likelihood of these things happening, as they hadn't in the past. ResN08 highlighted the pay disparity between ResNs compared to nurse educators and ACNMs despite similar levels of responsibility and expertise. While there was support for a career pathway, ResNs and NMs were uncertain how this would fit in with other clinical areas, because the ResN role in ICU was perceived as different to other areas by three ResNs and one NM. Another avenue expressed as a future direction $(n=3)$ was ResNs doing their own research, ResN06 expressing that "we've got the expertise" (L. 1616). There was acknowledgement that the development and acceptance of the position statement, recognition as a subgroup of the critical care section of NZNO and the meetings and networking within NZ had been a 'good start' $(n=3)$, and that working together was important in the future.

\section{Summary}

The thematic findings presented in this chapter have collectively shown the importance of the role, and the components that ResNs do in the ICU setting. Project management of every part of the trial process was a significant part of the ResNs work. The finding that ResNs had a significant role to play in patient and trial advocacy added to the third finding that they bridged gaps in knowledge and relationships between research, patients, their families and other healthcare professionals. Additionally, important attributes which enabled them to do these roles well, and barriers to performing their role were identified. These themes, in addition to 
the findings about demographic data, structures and background about the role of the ResN presented in Chapter 5, give a picture of the ResN role as a whole.

The next and final chapter draws these findings together, and reflects on them in the context of the literature and all of the information provided in the preceding chapters. 


\section{Chapter 7 Discussion}

\section{Introduction}

This study aimed to describe the role of ResNs in NZ Level III ICUs and the structures and funding arrangements they work within. An in-depth description of the ResN role was achieved by interviewing ResNs, PIs and NMs. The structures pertaining to ResNs, including line accountability and key relationships were described. The objectives included describing the ICU ResNs in NZ, identifying important components of their role and assessing the similarities and differences about the role. This chapter outlines key findings, and then discusses the three main findings in more depth. These findings are: The significance of the ResN role in trial management and 'making research happen' in ICUs, patient advocacy, and employment issues. Strengths and limitations of the study and the research design and process are then reflected on, particularly the use of the responsibility assignment matrix (RAM) as an analysis tool. Finally, practice, educational and research recommendations drawn from this study are made.

\section{Overview of findings}

This study interviewed ResNs $(n=11)$, PIs $(n=6)$ and NMs $(n=6)$ from six NZ ICUs about the role of the ResN. The ResNs were all female, well-educated and were an experienced workforce both in ICU and research. They had a commitment to ongoing education and two were conducting their own studies. All of the ResNs had learned how to do their job 'on the job' - five from senior ResNs in their department and six were self-taught. There were four different management structures within the research departments (Figure 6, p. 74), although the ResNs predominantly reported to a nursing or service manager $(n=10)$. Only three ResNs had a job description which was reflective of their role, and only five had a current performance appraisal. There was variability in the way ICU research departments were funded, which came from the ICU operational budget, studies and commercial sponsorship. All ICUs received some commercial funding either through unconditional grants or by participating in commercial trials. ICUs participated predominantly in investigator-initiated, grant funded studies, of which half were CTG endorsed.

There was almost universal agreement amongst ResNs, PIs and NMs that the primary role of ResNs in NZ ICUs was managing clinical trials. They had input into every stage of the 
research process including protocol design, deciding which studies ICUs would participate in, and setting up and safely managing trials through protocol adherence. ResNs worked in partnership with PIs on the daily management of clinical trials. All ResNs had some role in the consent process, either as the first person to approach a family about a trial or obtaining full consent. Individual hospital policy, PIs and ResNs determined how involved the ResNs were with the consent process.

ResNs were seen as the link between patients, bedside nurses and research. They balanced the needs of the trial with the needs of the patient using their dual specialty knowledge of ICU and research. Patient advocacy was synonymous with patient safety, which underpinned all of the ResNs' work. ResNs advocated for patients at every stage of research from the protocol design to ensuring the protocol was adhered to, and this advocacy ensured the patients' health needs had the highest priority.

ResNs were generally satisfied with their role, however, along with PIs and NMs cited overcoming a negative research culture within their ICUs as a problem. Additionally there was inconsistency in the expectations of ResNs regarding after hours availability and dissatisfaction about recognition and remuneration. There was variability about whether ResNs were considered senior nurses within ICU or not, although if they were, this was usually related to their ICU role or experience, rather than their research role. Research departments were regarded as being 'to the side' of ICU.

\section{Trial management - the research nurse's primary role}

The role of the ResN has been shown to predominately be managing clinical trials, which is in line with international literature (Bevans et al., 2011; Catania et al., 2012; Hill \& MacArthur, 2006; Jeong et al., 2007; Mori et al., 2007; Mueller, 2001; Nagel et al., 2010; Rickard et al., 2006; Rico-Villademoros et al., 2004; Roberts et al., 2011b). ResNs were like the conductor of an orchestra, who direct the parts to create the 'whole'. Conductors ensure that everyone knows the part they have to play, and ensures they play it correctly so that the music is brought together harmoniously. Like the conductor, ResNs knew what had to be done, when it had to be done and by whom. They also understood why it had to be done, and in some cases, they did the task, and in all other cases they facilitated it. ResNs became experts on individual trials 
and probably knew more about the practical aspects of conducting the study than any other person involved.

The components of the ResN's role were in alignment with the international literature, with a focus on administering the protocol, protocol adherence, data collection, patient follow-up, as well as consenting, which is discussed later in this chapter. The ResNs were responsible for the ethical components of trial set-up including writing ethics submissions, information sheets and consent forms. They also facilitated contract negotiations, liaised with other departments and educated nurses (and sometimes doctors) about new trials. One important component of their daily work which kept emerging was the importance of screening and recruitment for studies. Where ICUs were funded by studies, recruitment was essential to generate funding. This made the ResNs reliant on other staff because recruitment into studies in ICU was a 24 hour a day job. Recruitment was previously only articulated in the literature by Hill and MacArthur (2006) and Roberts et al. (2011b). Hill and MacArthur also reported that ResNs were dependent on recruiting patients to pay their salary.

Research tasks were divided between ResNs and PIs according to practicalities such as availability and expertise. This division of labour can be seen as the delegation of duties referred to by Mueller (2001) and Mueller and Mamo (2002) but also as a practical application of teamwork in the healthcare setting (Opie, 1999). Teamwork in research is not only practically advantageous, but offers patients the best that professional groups have to offer. The finding that nurses had different knowledge to doctors, who tended to have a more scientific approach was similar to the findings in a paper by Snelgrove and Hughes (2000). These authors identified nursing knowledge as patient advocacy and holistic care which were similar to the findings in this study. When PIs spoke of the ResN, they appreciated the practical elements and expertise they brought to the role; however they never spoke about more nursing-focussed activities such as patient advocacy. Similar to the findings in Anderson (2008), ResNs worked in partnership with PIs and there was significant trust between them. The PI trusted the ResNs to do some difficult trial-related tasks, which they would then "sign off'. ResNs respected that trust and would seek their guidance and help when required as seen in Mueller and Mamo (2002). The ResNs had a close and autonomous working relationship with the PI, who held the ResNs in the very highest regard and were their biggest advocates. They worked with ResNs and delegated responsibilities such as obtaining consent to them, as 
the ResNs became more experienced. While this was reminiscent of Mueller and Mamo's (2000) finding that study duties were delegated along socially-stratified lines from higher (doctor) to lower (nurse), the close working relationship described above was suggestive of a collegial and collaborative division of labour according to skill set and work expectations. ResNs were solely responsible for the day-to-day management of the trials, which was not delegated, but was considered their work. The RAM analysis often had both the PI and ResN being 'accountable' for a task. In a management setting this might be concerning (Smith \& Erwin, 2007), however in the context of the ResN and PI working in a partnership this was an understandable finding. This close nurse-doctor relationship is reportedly more common in specialised areas such as ICU, where nurses are seen as having specialised knowledge (Chaboyer \& Patterson, 2001; Coombs \& Ersser, 2004).

The role of the ResN was identified as essential for ICUs participating in research. While some of the reasons may have been pragmatic, it was also recognised that ResNs had extensive skills and experience, both in ICU and research. The ResN was the 'face of research' for nurses and patients, and 'made research happen' on a daily basis. To achieve this, they needed to be skilled and knowledgeable. There was resistance from ResNs to being seen as "just data collectors". While all acknowledged this was an important part of their role, and in some cases was seen as essential for respecting the patients' contribution, ResNs did not want to be defined by this. Perhaps this side of the role is all that many clinical staff see, but their role was so much more. ResNs were clearly not 'handmaidens', and were not 'doing doctors work', although it was highlighted that few were leading their own research, which is an area that may be developed in the future.

The ResNs' contribution to patient care is perhaps best viewed as a long-term investment. In the time that I have worked as a ResN, the ICU I work in has contributed to two significant studies which, when published changed or reaffirmed practice in ICU. The first of these compared tightly controlled blood glucose levels with a more liberal approach (NICE Study Investigators, 2009). The study showed that ICU patients who had their blood glucose levels tightly controlled by insulin had a higher mortality rate, at the 90 day follow up. Another study showed that early decompressive craniectomy in patients with a severe traumatic brain injury worsened the functional outcome of these patients (Cooper et al., 2011). These studies 
highlight the significant contribution ICU research can make to improving patient care and outcomes.

Many ResNs and PIs spoke about their frustrations with medical and nursing staff within their ICUs, and a 'lack of a research culture' was reported in four ICUs. This was reflective of the study by Spilsbury et al. (2008) where management had agreed to participate in a trial, then the ward staff were uncooperative with study treatment. In the case of ICUs what is interesting is that the CICM Minimum Standards of Intensive Care Medicine (CICM, 2010b) state that level III ICUs should have an "active research programme" and "employ a research coordinator". Additionally, this guideline states that "the majority of nursing staff will have a post registration qualification in intensive care or the specialty of the unit" (p. 2) and must have a "formal nursing education program" (p. 2). On the basis of the Minimum Standard guideline, it could be assumed that all nurses and intensivists would want research to occur in their ICUs, yet their lack of cooperation was cited as a barrier to recruiting patients into research studies. Johnson (2008) completed a thesis on the perspectives of nurses towards clinical trials in one NZ ICU. Johnson's study reported that bedside nurses needed more support regarding education and trial implementation, because clinical trials impacted on their workload. The nurses also raised ethical concerns about consent procedures. Without speaking to (nonresearch) intensivists, it is impossible to determine why they don't fully participate in studies, even though their ICU has agreed to participate.

\section{Patient advocacy}

While all staff involved in research act as ethical gatekeepers, ResNs played a particularly significant role in advocating for patients. The ResNs in this study acted as patient advocates throughout the entire research process. While Davis (2002) importantly found that study coordinators had a key role in balancing the needs of the trial, the patient and the patient as a study subject, this research has shown that advocacy extends beyond what is done clinically in research. In participating at the development stage of trial protocols, ResNs were able to advocate for the patient, but also for the study, by identifying study components that would and wouldn't work, or would be too difficult in the ICU setting. They were experts in the practicalities of conducting clinical research in this challenging environment, and their involvement enabled trials to start with a clear and practical protocol. 
To be a patient advocate, ResNs needed to use their clinical judgement and experience to decide which patients were suitable for clinical trials from a holistic point of view, taking into account human elements beyond a trial's inclusion and exclusion criteria. This tendency to advocate for the patient is an inherent part of nursing (Fouka \& Mantzorou, 2011; MacDonald, 2007; Nursing Council of New Zealand, 2012); however, perhaps it is also good for clinical trials. Although Chatfield (2008) states that the quality of research is improved if nurses are involved, we don't really know what effect the ResN role has on clinical trials. ResNs had a significant role in protocol adherence, which adds to the overall quality of studies and the data collected. It is possible that their involvement may reduce the number of patients who decline consent, are 'lost to follow-up' or were assessed as unsuitable for enrolment in the first place because of the constant checking and monitoring they do.

Another layer of advocacy identified was that ResNs 'bridged knowledge gaps'. This was most significant for the bedside nurse, and patients and/or families. Providing information in a way that patients and families understood provided them with the opportunity to make an informed decision about participating in trials. 'Translating' medical information for families is an important role for ICU nurses (Coombs \& Ersser, 2004) and communicating information about studies to bedside nurses and associate charge nurse managers (ACNMs) contributed to good conduct of clinical trials and adherence to the protocol. ResNs have been variously described in the literature as 'the hub' (Davis et al., 2002) and the 'glue' (Duane et al., 2007), and the findings in this study are congruent with these studies. ResNs had a key relationship with the bedside nurse, assisting with trial duties, and educating them about aspects of trials. This finding is in contrast to those reported in Johnson (2008).

ResNs had extensive knowledge about ethics - both at the bedside advocating for patients, and the procedural requirements of ethics committees. ICU patients are extremely vulnerable and ResNs took this responsibility seriously. This study provides important findings about the role of ResNs in the consent process. The consent process was the area which showed the most difference across the ICUs. The level of involvement with regard to consent varied according to the institution ResNs worked in and their interpretation of guiding documents. What this study has done is articulate that there are different levels of risk associated with different clinical trials, and in some cases ResNs were able to obtain consent from patients and/or their families. When ResNs did obtain consent for trials, it was generally for 'low risk' studies 
where two existing standard treatments were being compared. In particular, ICUs that were participating in the CHEST trial (The CHEST Management Committee, 2011), which was considered lower risk, had recent experience of ResNs obtaining written consents. Considering the trials that ResNs in the Australian literature were participating in were likely to be similar or the same CTG-endorsed studies, it is not surprising that this finding was similar to those studies (Rickard et al., 2006; Roberts et al., 2011b), in which 78 - 80\% of ResNs obtained consent. It was unclear how involved ResNs were in the consent process in other studies (Bevans et al., 2011; Catania et al., 2008; Nagel et al., 2010). Informed consent has traditionally been the domain of the medical profession. The more studies that ICUs participated in, the more 'routine' obtaining of consent became and so ResNs' obtaining consent could be seen as a task delegated 'down' from doctors to nurses (Mueller, 2001). Other reasons identified for ResNs obtaining these consents were that they had more time, and had extensive knowledge about trials. In addition, as ResNs became more experienced, they may have developed the expertise and confidence necessary. Some ResNs went as far as to say they were more familiar with the protocol than many non-research doctors, and were sceptical about non-research doctors completing consents. By enrolling patients into studies and obtaining consents correctly, ResNs could be seen as straddling medical and nursing work as Mueller suggests.

ResNs and PIs used their judgement to decide the most appropriate time to obtain ongoing consent, following entry into a study with delayed consent or assent. ResNs also used their experience and judgement to decide if it would be more appropriate for a doctor to talk to a patient or family, in some situations. As long as ResNs are fully aware of any risks associated with consenting patients into clinical trials and are acting within accordance of their professional responsibilities, these institutional and personal beliefs must be respected.

The 'Guideline on the Regulation of Therapeutic Products in NZ' states that "supplying clinical trial medicines to trial participants is not considered to be prescribing or dispensing" (MOH, 2011, p. 20), however the relevance of this to the ICU setting is unknown. There is no doubt that consent in the ICU setting is a complex ethical situation, to which both doctors and nurses contribute. ResNs, even those who do not obtain consent for some or all ICU studies, participate in the process, by ensuring that it is done correctly and ethically. What would happen to this process if ResNs were replaced with data collectors? The area of consent in 
unconscious patients is largely untested (MOH, 2006), and ResNs must continue to be ethical gatekeepers. It is unknown what the role of ResNs in other clinical areas is in obtaining consent for clinical trials.

\section{Employment issues}

ResNs were a highly skilled and experienced workforce, yet despite this, many thought this work was poorly recognised. All ResNs did most of their learning 'on the job', not unlike apprentices, although eight had attended formal GCP training through external agencies or their hospitals. The finding that newer ResNs were trained by more experienced ResNs in their departments is pleasing as it reveals the depth of experience that is now available to new ResNs. Apprenticeship type training is, however, dependent on the opportunities that arise, a degree of trial and error, and the skills of the trainers.

The finding that eight (73\%) of the ResNs did not have an accurate job description, and three had never had a formal performance appraisal for their research position highlights a major issue. A similar problem was identified by both Hill and MacArthur (2006) and Rickard et al. (2006) where $28 \%$ and $31 \%$ respectively did not have job descriptions. In these studies, there was also concern that ResNs did not have permanent contracts, which was identified as a reason for not having a job description. The difference in the NZ setting is that virtually all of the ResNs studied were employed on permanent contracts (or were soon after the interviews), yet $73 \%$ did not have an accurate job description. The reasons for no job description were not identified; however their absence highlights risk and safety issues both to the individual and the hospital. If anything ever went wrong with a patient in a research trial, this could be problematic for an individual ResN and the hospital. Not having an accurate job description also adds to role confusion, even more so when ResNs were employed for one reason, and then their job changed. Equally difficult was where there was no chance of a ResN completing tasks such as quality and audit within their funded FTE. This study was conducted during a time of change, and I am aware that some ICUs were updating job descriptions. This lack of clarity about the role in some ICUs would make it difficult to conduct a performance appraisal. On top of this, ResNs expressed that their NMs didn't know in detail what they did and had no involvement in the day-to-day management of the research departments. In most cases, the NM was their line manager, who they were professionally accountable to and who was responsible for their appraisal. 
ResNs enjoyed their autonomy and did not suggest that NMs became more involved with the day-to-day management of research. While some NMs had limited knowledge about specific aspects of research, others had considerable knowledge about many parts of the ResN role and their work. Most NMs took the stance that as senior nurses ResNs were capable of working independently and were responsible for the day-to-day work which had been delegated to them. This could be considered an appropriate stance as perhaps the NMs were only involved with the management of ICU patients if a problem arose. The question is, to what extent do NMs need to know about the role of the ResN and how much should be known for line management?

It was not an objective of this study to advocate one structure over another; however, the study did highlight the importance of a shared understanding amongst those in key positions about lines of accountability and responsibilities. There was not always agreement between participant groups about the management structure that ResNs worked in. The reason for this is unknown and because the ResNs did not have current job descriptions, it is difficult to assess the most accurate viewpoint. One possibility is that in some ICUs, an informal structure coexists along with a formal structure. While the official accountability for all but one ResN was a nursing manager, over half of ResNs also reported an informal accountability to the PI. This differed from the literature in which only 16 - 17\% had a nursing line management (Hill \& MacArthur, 2006; Rickard et al., 2006), although Rickard et al. reported dual line management with the PI in a further $18 \%$. The appropriateness of the NM being the ResNs line manager was questioned by one PI - a view that was also inferred by the informal accountability with PIs, reported by ResNs. While it was unclear whether the NM was the most appropriate line manager, the question about who would be an appropriate line manager is then raised. What is of utmost concern is that a two-tiered (formal and informal), dual accountability (NM and PI) structure was the most common structure in use. Dual accountability and unclear lines of accountability are very confusing. The problem with dual accountability is that it has the potential to mean no accountability, direction or leadership. There would be professional accountability implications for a nurse having a doctor as a manager although this was the management structure for one ICU. 
Despite ResNs being employed on the collective agreement as senior nurses (DHB/NZNO, 2012) another area of concern was the ambiguity reported by NMs about whether ResNs were senior nurses in ICU and the suggestion that they were considered senior nurses 'in name only'. This was not a view shared by most PIs, who had the greatest respect for the contribution ResNs made. ResNs were (generally) expected to perform their duty of care if an ICU patient experienced a clinical emergency, yet weren't necessarily considered a senior nurse operationally. Despite this, NMs expected ResNs to manage their own workload, be autonomous and professional. While ResNs did not identify themselves as feeling isolated, in many ICUs, research was seen as 'separate' from ICU. It was unclear whether the 'separateness' of the research departments was beneficial or not although it contributed to the role of ResN not being seen as part of the senior team within some ICUs. While much of the work ResNs did in ICU was seen as clinical, there was still a need, either self-imposed or contractual, to be seen as 'credible' with nursing staff by doing clinical shifts as a bedside nurse. This suggests that, in the absence of doing clinical or bedside nursing, the ResN role was not seen as contributing to patient care, which is not congruent with the findings of this study. It would be of value to see if the bedside nurses agreed with this finding and whether they think that nurses who have 'stepped away from the bedside' are no longer nurses, or if it's an "old notion" as suggested by one NM.

Many ResNs were protective about their set research hours, citing reasons such as funding as a reason not to help clinically when the ICU was short-staffed. This stance has to be viewed in the larger context and that in most ICUs ResNs received no help with their research workload. The ResNs took their role and responsibilities seriously and were prepared to do nearly all research activities for a patient who was enrolled in a trial including phoning from home to check on them or come in out of hours when a problem occurred. When they did work clinical shifts, they continued to do research duties where possible, while also looking after an ICU patient.

The reported 'separateness' is significant not only because ResNs are working in ICU and doing research, but also for their career pathway. Only one ICU had a formal career pathway, although there was an informal hierarchy in others where a more senior ResN delegated tasks to a junior. Having no career pathway meant that no matter how experienced ResNs were, their grade and pay remained the same. As seen in the ICU which had developed a career 
pathway, this could be developed at a local level, and did not rely on a national collective agreement. However, in order to achieve this, the role needed to recognised as important, which was not always the case. ResNs clearly have expert skills in a wide variety of studyrelated activities including clinical, ethical and financial. In order to retain this depth of knowledge, a career pathway must be considered to ensure experienced ResNs are retained in these positions, an issue recognised by Leonie Walker, a researcher at NZNO (Walker, 2009a, 2009b, 2010). Although the ResN role has arisen out of a need to have 'someone' taking responsibility for managing clinical trials in ICU, it is an important role and its origins as work delegated by doctors does not preclude the role from becoming a specialised position. Bevans et al. (2011) described a pathway of sorts, which defined clinical research nurses in a large research centre as those that delivered care to patients, while research nurse coordinators focussed on the whole process including study coordination. As this role has been identified as a nursing role in the NZ setting, it is imperative that Directors of Nursing and other nurse leaders understand the significant work that ResNs do, and acknowledge the importance of it by establishing a defined career pathway with an appropriate salary scale. While PIs recognise that without ResNs there would be no research, nurse leaders have yet to acknowledge the importance of this role.

Remuneration was a source of dissatisfaction for some ResNs. This was because they were reportedly on a lower 'grade' than other senior nurses, including ACNMs and educators in ICU, despite having similar levels of ICU experience. Some ResNs had also been 'downgraded' in the past, when the role was re-scoped by NZNO. The pay one receives can be seen as a form of recognition. Lack of recognition was reported by Roberts et al. (2011a) as the 'worst' aspect of the role and dissatisfaction about pay was similar to the findings in their earlier studies (Roberts et al., 2006). When I contacted the NZNO about the pay structure, their only suggestion was to apply for the role to be re-scoped at a local level through the "Job Evaluation Review Committee" (DHB Shared Services, 2012). More work needs to be done collectively amongst ResNs if the job is to be re-scoped to a higher grade, as in the past rescoping had not worked in the ResNs' favour.

The need for an on call system varied depending on the particular trials that units were participating in. ResNs generally did not express dissatisfaction about their on-call arrangements, because they had developed informal systems which worked for their ICU and 
possibly because they didn't know what was happening in other NZ ICUs. However, some of these arrangements were problematic because they relied on the goodwill of ResNs, and as such are unsustainable in the long term. ResNs in three ICUs were not paid to be on-call, yet there was a tacit understanding that they would be available to come in after hours to enrol patients into studies. Goodwill and tacit understandings are problematic and mean the ResN who can't respond to such requests could be vulnerable. It also means the ability of the ICU to enrol patients is at risk. The Australian ICU studies (Rickard et al., 2007; Roberts et al., 2011a; Roberts et al., 2006) reported on call arrangements as a source of dissatisfaction. ResNs must be involved in any changes to on call arrangements because one ResN who was paid to be on call was dissatisfied, and an appealing aspect of the role for others was the 'flexible hours'. For most ResNs, this was not a job which could be left behind at the end of the day. In many cases, ResNs worked above and beyond the terms of their contract to ensure protocol adherence both for the patient and the trial, by phoning and checking on trial patients after hours and during their days off. In this context, the bitterness about downgrading of ResNs pay rates and dissatisfaction with pay can be understood.

Funding was an issue for half of research departments which meant that the job security of some ResNs was tenuous. There was a mix of how ICU research departments were funded, and while commercial sponsors and ICU operational funding added to the overall security of the research department, the ResNs' sense of security was equally related to the research culture of the ICU they worked in. The two PIs who were unconcerned about finances had an unconditional ongoing grant from an external commercial sponsor. In these difficult financial times, more public/private partnerships may need to be considered for future funding for research. However, careful consideration must be made about how research remain rigorous and independent if there is a dependence on commercial funding, before embarking on this route. There was widespread belief amongst ResNs and PIs that research should at least be partly funded by the government or DHB. The NMs were more pragmatic about this, and thought any additional health spending would be better spent operationally rather than on research. This disagreement between nursing and medicine is of concern, particularly given the dual accountability that ResNs had to them.

The network established by NZ ICU ResNs themselves is an important forum for future development of the role. While many employment issues were decided locally, a collective 
national voice would raise the profile of ResNs, and a shared understanding about the role would help all ICU staff to understand it better. There was widespread acknowledgement that initiatives already underway such as the draft National Competency Framework for Clinical Research Nurses (Walker et al., 2010), were an important first step for all ResNs.

\section{Strengths and limitations of this study}

This study is the first of its kind in NZ describing ResNs in the ICU setting. It draws on the views of multiple stakeholders and $100 \%$ of invited participants agreed to be interviewed. This has enabled triangulation of data within ICUs and across professional groups to provide a balanced role description. Early consultation with ResNs about this study and being known to participants enhanced recruitment and was also important for informing what to ask. Being an 'insider' in this study also proved to be advantageous and was a strength. When participants talked about specific trials or parts of the role, I understood what they were talking about and was able to concentrate on what they were saying. Additionally, people were interested in the study, were open with their answers, and the range of views provided indicates they probably answered honestly. This thesis has presented a detailed methodology chapter which highlights rigour strategies employed such as member checking, triangulation and audit trail. For example, relevant quotations with line numbers are evidence of the audit trail.

In the main, limitations are an inherent part of the research question, which had a limited scope of ResNs in the NZ ICU setting. Generalisability to settings other than ICU was never an aim for this study, or methodology, although describing the role within this setting adds to the body of knowledge about ResNs generally. While ICU patients are extremely ill, and it could be assumed that they are the 'sickest of the sick', patients are also extremely sick in areas such cardiology and oncology, and with different requirements, so it is possible that some of the findings could be transferable to other clinical areas.

\section{Reflection on methods of inquiry}

Two main methods were used for this study - semi-structured interviews and RAM. While both of these tools had advantages and limitations, the overall data were comprehensive and rich. I would recommend the use of both of these methods in any future research about the topic, however some considerations are needed when using the RAM in this type of study again. 


\section{Interviews}

Using semi-structured interviews was a sound method as the data elicited were rich and provided good information about the role of the ResN. However, there are always ways to improve the way we do things and this section reflects on what I learnt from my first interviewing experience.

The technique of using closed questions first to establish rapport with participants worked well with ResNs and NMs, but less so PIs, who were generally very busy. One of the background questions intended to establish rapport with the PIs was 'Can you tell me which major studies you have been involved in, and your role, in the past?' One PI, who had been involved in many research studies before, offered me their curriculum vitae with a list in lieu of answering this question. This question had the opposite effect on the interview, and it took some time for me to settle again. Because of other commitments, two PIs imposed time limits for the interview, and another had to eat their lunch during the interview. Because of these time limitations, I made judgements about which questions were essential. While all main points were covered, thorough discussion about some topics was limited. Next time, I would consider a shorter interview schedule.

Semi-structured interviewing allowed for participants to talk about topics they thought were important. It also meant that interviews often proceeded in a different order to the interview schedule and the odd question was omitted or asked differently. Changes to the way I asked some questions also evolved throughout the interviews, although ultimately this did not affect the data collected. The way answers were elicited from the three groups also differed. The ResNs were generally happy to talk around topics, whereas the PIs and NMs tended to just answer the questions asked of them. There was an inclination in all of the interviews for the participants to talk about their own role in research, rather than that of the ResN. This was to be expected and most attempts made to bring the interview back to the ResN were successful.

I took the stance not to question participants' answers which I believe I achieved. However, questions about which studies ICUs were participating in became more of a statement than a question in some of the PI and NM interviews. In some interviews, the participant didn't answer a question, even with prompting. It was difficult to determine the meaning of this. For example did they not know the answer, did they not understand the question or did they 
disagree with what was stated or did they just not get around to saying it, as I had prompted them too soon? I did however ask for examples and not just opinions.

ResNs who were more experienced and senior spoke with more clarity about the role, and were able to identify a range of examples, compared to those who had been in the role for a relatively short period of time. While this clarity was useful, care was taken to give newer ResNs' views the same weighting as their more experienced colleagues.

There was little time in-between interviews, which was confusing at times, and meant I relied heavily on the interview schedules to 'keep on track'. The use of recordings meant I could distinguish responses without reliance on my field notes or memory of the interviews. This was unavoidable due to time constraints of the participants and my own budgetary limitations, and while I don't think it affected the overall quality of the study, I would not recommend that such a tight timeframe is used for future studies of this type.

One ResN said she was disappointed that I hadn't asked them to complete the time-in-motion diaries, as she found it difficult to recall her previous day, citing how busy the role was, and how different the days can be. This is a valid point, however most ResNs were at least able to describe a 'typical' day in detail. Another method which had been considered was a document analysis of the ResNs' job descriptions. While this would not have made a difference to this study, as ResNs reported they did not have accurate job descriptions, it would be a useful adjunct, when writing about the management structures and I would recommend this for future research.

One aspect of the ResN's role which I did not directly address on the interview schedule and did not arise in the interviews was whether ResNs were named in publications. This was an oversight as it was noted as an issue in the literature review. I already knew before starting this study that PIs and ResNs who are involved in CTG-endorsed studies are usually listed at the back of publications - a stance which must be commended by authors who do this. ResNs are only listed as a named author if they are on the management committee for a study, of whom five ResNs were. I am unaware of ResNs being named on publications resulting from other studies, such as commercial trials. I perhaps incorrectly interpreted that if ResNs did not bring this issue up, then they did not view it as important. 


\section{Responsibility assignment matrix}

Using the RAM was a novel addition to analysing the work that ResNs do. Overall, its use contributed to the study. In some cases, not all questions related to the RAM had been asked and in some cases the participants misunderstood the terms used or did not know or understand the specific tasks the ResNs did. This was evident in the NM interviews. Adding 'Unknown' as an option was a useful modification for this research.

Analysing data using the RAM from a qualitative method, such as one-to-one interviews was difficult. One of the limitations of this particular model was that it didn't reflect the extent to which an activity occurred. An example of this was study recruitment. When participants answered that particular professional groups recruited patients for studies "very occasionally" and "seldom" there was no way of articulating this degree of engagement on the matrix. In the case of consent, all participants stated that non-research intensivists obtained patient consents, in addition to the research team. On the RAM, this looks like they actively do, whereas the qualitative data from the interviews revealed consents were obtained almost exclusively by the ResN or PI in some ICUs.

A short-coming in my own use of the RAM was that it was designed as an analysis tool, and I did not validate it before the completed matrices were sent to participants for verification. Only later did I realise that 'protocol development and design' was not listed and so the data collected in the interviews were used in the final results.

A modification of the way the tool was used would have been to send the RAM out for the participants to fill in themselves. Given that $93 \%(\mathrm{n}=13)$ of those who returned their RAM made changes, this may have been an easier option, however; there was potential for low return rates and misinterpretation of the tool and tasks. A disadvantage of asking participants to review their RAMs was that collaboration appears to have occurred amongst participants at some ICUs. Although this may have resulted in data changing, enabling a shared understanding amongst participant groups must be seen as positive. The use of the RAM, along with qualitative data collection added to the overall depth of understanding, and I would recommend its use in a similar study. 


\section{Recommendations}

\section{Practice recommendations}

Issues regarding employment conditions such as remuneration, on-call payments and line management must be addressed. Ensuring ResNs have an accurate job description and current job description is the responsibility of both the individual ResN and of the organisation. Some ResNs need to find out if they have a job description, read it, and determine its accuracy. If it is not accurate, this must be addressed urgently with management. ResNs must also ensure their own professional safety in the domain of consent.

Nursing management in consultation with medical staff, and DHBs' legal services must address the issue of professional accountability, including indemnity, and who is responsible if something goes wrong in a clinical trial. ResNs should not be put at risk by assuming tasks which were once the domain of doctors.

While less urgent, the ambiguity about whether the ResN role was a senior position must be addressed by the sector, in order to retain this experienced workforce. ResNs had specialist knowledge (about research) in an already specialised clinical area (ICU) and should not be side-lined because management are uncertain where the role fits into the overall structure.

The recommendation that all CTG-endorsed studies have a 'research coordinator' on the management committee is very sound and must continue. It allows studies to succeed in a practical, rather than just theoretical way. ResNs were also involved in reviewing study documents, to ensure the smooth-running of studies. The CTG must be commended for these initiatives which logically increase the success and quality of CTG-endorsed studies.

\section{Educational recommendations}

As the number of ResNs increase, the appropriateness of the 'apprentice' style of learning about their role must be addressed. In addition to the research opportunities at universities that offer postgraduate studies in nursing, there is now a Postgraduate Diploma in Clinical Research (Victoria University of Wellington, n.s.) and a clinical research postgraduate pathway through to Masters level at University of Otago (University of Otago, n.s.). These options may offer an avenue for ResNs to pursue academically in the future. All those who don't yet have a Masters degree should be supported to do one, and where possible PhDs should be encouraged. 
Few professional development opportunities for ResNs were identified. When these opportunities do arise, NMs must support ResNs to attend to ensure the ongoing success of research in ICU. The experience amongst ICU ResNs throughout NZ is now considerable, and they themselves have established a network, to support newer members. This work needs to continue, and I am aware that there is development of a website affiliated with the NZNO Critical Nurses Section. These opportunities need to be embraced to ensure that ResNs are seen as a cohesive group.

\section{Research recommendations}

Several avenues for further research were identified throughout this thesis. These concern the issue of consent, research culture, knowledge translation and other ICU professionals' views about research issues in ICU.

The area of consent was contentious, and very reliant upon research staff acting ethically and in the best interests of the patients. I believe research staff in NZ ICUs do act ethically; however it would be useful to see how patients feel when they are de-sedated after a prolonged stay in ICU only to find they have participated in a research study. Delayed consent is considered essential for conducting research in ICU. A future study should investigate how often it is used in this setting, studying the time it takes to obtain full consent and the number of patients who withdraw from trials. It would be important to see if other staff treated the option of 'delayed' consent with as much care as research staff, who seemed to understand the value of this ability to enrol patients into ICU studies without their prior consent was. Another study should also ask patients how they feel when a study shows 'no result' or worse still a negative result, or a positive result when they were in the control group.

Incorporating bedside nurses and non-PI doctors would be a useful adjunct to this study as their viewpoint has not been heard. Although this study has extended the boundaries of what is known by seeking the views of the NM and PI, it highlights that ResNs work with many other professional disciplines within ICU. Lack of research culture in ICUs was a significant problem and must be investigated in the future. It would be useful to know what other intensivists and nurses in the ICU setting think about research and find out why they didn't participate more actively. What are the barriers to conducting research? Do staff nurses and non-research doctors even consider it part of their role? 
As already stated, this thesis is limited to the ICU setting. It would be useful to compare their role with research nurses working in other settings such as primary and secondary care, academic settings and private research companies. Additionally, the majority of ResNs assisted and supported nursing research - a future project could explore the barriers and enablers fpr ResNs supporting nursing research.

Future research must also, and to some extent already is, focus on the financial and social savings benefits of ICUs participating in research. Additionally, work must focus on translating these research findings into ICU patient care, thus improving outcomes for intensive care patients. The role of the ResN in such practice development work should be explored. Finally, the increased value ResNs add to this research through increased recruitment and having a dedicated role should be included in these studies.

\section{Conclusion}

When I began this research, my starting point was my own experience, seeking answers to whether my experiences were similar to those of other ResNs. As an insider in a role which participates in research, seeking answers through research was a good solution for my questions. I have learned so much along the way. By conducting this research, I have learned how to do my role better, and I understand the larger environment we work within. Some of my experiences were similar to others, while other ResNs had taken a different path to arrive at their individual destination. What was clear was that they all wanted the same thing, and that was to participate in research with the potential to improve the outcomes of ICU patients in a way they saw as valuable.

This study will enable ResNs, their PIs and NMs in ICUs around NZ to have a more global picture of the role. There was already literature about the ResN role, but where this study goes further, is it has described and given specific examples of exactly how ResNs contribute to research in ICU. This research, in turn, improves outcomes for future ICU patients. This is an important study which celebrates the significant work that ResNs do in NZ ICUs. It demonstrates that they are not only the glue that holds ICU research together but they 'make research happen' on a daily basis. ResNs enable ICUs to participate in research which is imperative not only for improving patient outcomes for ICU patients, but to ensure Level III 
ICUs fulfil their obligation to be involved in research. ResNs ensure that patients and their families are offered the opportunity to improve the care of future patients. Major issues that ResNs encounter have been identified, and this will pave the way to enhancing and developing this role. 


\section{Appendices}

\section{Appendix A Summary of quality of literature using STROBE criteria}

\begin{tabular}{|c|c|c|c|c|c|c|c|c|c|c|c|c|c|c|c|c|c|c|c|c|c|c|c|c|c|}
\hline \multirow[b]{2}{*}{ Author } & \multirow[b]{2}{*}{ Year } & & \multirow[b]{2}{*}{1} & \multicolumn{2}{|c|}{ Intro } & \multicolumn{9}{|c|}{ Methods } & \multicolumn{5}{|c|}{ Results } & \multicolumn{4}{|c|}{ Discussion } & \multirow[b]{2}{*}{22} & \\
\hline & & & & 2 & 3 & 4 & 5 & 6 & 7 & 8 & 9 & 10 & 11 & 12 & 13 & 14 & 15 & 16 & 17 & 18 & 19 & 20 & 21 & & \\
\hline & & 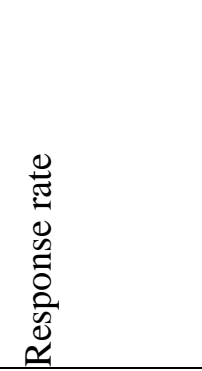 & 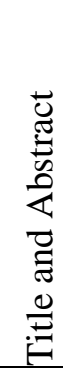 & 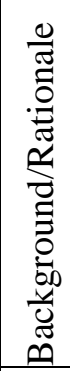 & $\begin{array}{l}\stackrel{0}{0} \\
.00 \\
0 \\
0\end{array}$ & 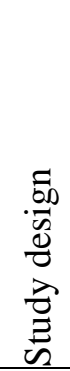 & 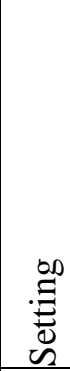 & 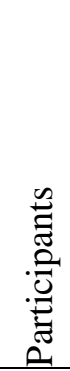 & 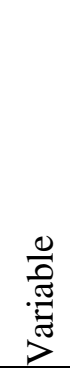 & 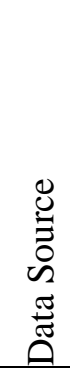 &.$\tilde{0}$ & 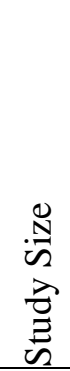 & 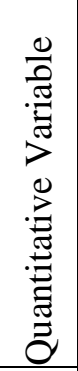 & 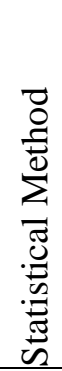 & 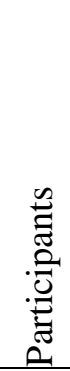 & 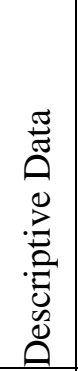 & $\begin{array}{l}\tilde{\Xi} \\
0 \\
0 \\
\Xi \\
0 \\
0 \\
0 \\
0\end{array}$ & 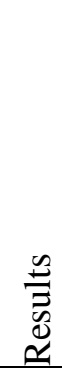 & 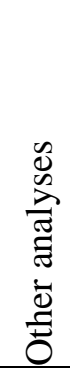 & 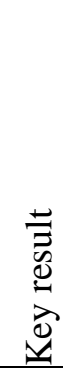 & .0.0 & 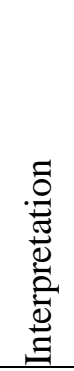 & 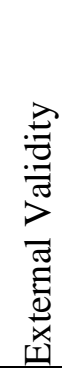 & 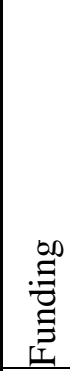 & ర్ \\
\hline Anderson & 2008 & $47 \%$ & $\mathrm{Y}$ & $\mathrm{Y}$ & $\mathrm{Y}$ & $\mathrm{Y}$ & $\mathrm{Y}$ & $\mathrm{Y}$ & $\mathrm{Y}$ & $\mathrm{Y}$ & $\mathrm{Y}$ & $\mathrm{Y}$ & $\mathrm{Y}$ & $\mathrm{Y}$ & $\mathrm{Y}$ & $\mathrm{Y}$ & $\mathrm{Y}$ & $\mathrm{Y}$ & $\mathrm{Y}$ & $\mathrm{Y}$ & $\mathrm{Y}$ & $\mathrm{Y}$ & $\mathrm{N}$ & $\mathrm{Y}$ & 21 \\
\hline Bevans et al. & 2011 & $41 \%$ & $\mathrm{Y}$ & $\mathrm{Y}$ & $\mathrm{Y}$ & $\mathrm{Y}$ & $\mathrm{Y}$ & $\mathrm{Y}$ & $\mathrm{Y}$ & $\mathrm{Y}$ & $\mathrm{Y}$ & $\mathrm{Y}$ & $\mathrm{Y}$ & $\mathrm{Y}$ & $\mathrm{Y}$ & $\mathrm{Y}$ & $Y$ & $\mathrm{Y}$ & $\mathrm{Y}$ & $\mathrm{Y}$ & $\mathrm{Y}$ & $\mathrm{Y}$ & $\mathrm{Y}$ & $\mathrm{Y}$ & 22 \\
\hline Catania et al. & 2012 & $88 \%$ & $\mathrm{Y}$ & $\mathrm{Y}$ & $\mathrm{Y}$ & $\mathrm{Y}$ & $\mathrm{Y}$ & $\mathrm{Y}$ & $\mathrm{Y}$ & $\mathrm{Y}$ & $\mathrm{Y}$ & $\mathrm{Y}$ & $\mathrm{Y}$ & $\mathrm{Y}$ & $\mathrm{Y}$ & $\mathrm{Y}$ & $\mathrm{Y}$ & $\mathrm{Y}$ & $\mathrm{Y}$ & $\mathrm{Y}$ & $\mathrm{Y}$ & $\mathrm{Y}$ & $\mathrm{Y}$ & $\mathrm{Y}$ & 22 \\
\hline Davis et al. & 2002 & $\mathrm{n} / \mathrm{a}$ & $\mathrm{N}$ & $\mathrm{Y}$ & $\mathrm{Y}$ & $\mathrm{Y}$ & $\mathrm{Y}$ & $\mathrm{Y}$ & $\mathrm{Y}$ & $\mathrm{Y}$ & $\mathrm{N}$ & $\mathrm{Y}$ & $\mathrm{N}$ & $\mathrm{N}$ & $\mathrm{Y}$ & $\mathrm{Y}$ & $\mathrm{Y}$ & $\mathrm{Y}$ & $\mathrm{Y}$ & $\mathrm{Y}$ & $\mathrm{N}$ & $\mathrm{Y}$ & $\mathrm{Y}$ & $\mathrm{Y}$ & 17 \\
\hline Duane et al. & 2007 & $83 \%^{1}$ & $\mathrm{~N}$ & $\mathrm{Y}$ & $\mathrm{Y}$ & $\mathrm{Y}$ & $\mathrm{Y}$ & $\mathrm{Y}$ & $\mathrm{N}$ & $\mathrm{N}$ & $\mathrm{N}$ & $\mathrm{Y}$ & $\mathrm{N}$ & $\mathrm{N}$ & $\mathrm{N}^{2}$ & $\mathrm{Y}$ & $\mathrm{Y}$ & $\mathrm{Y}$ & $\mathrm{N}$ & $\mathrm{Y}$ & $\mathrm{N}$ & $\mathrm{Y}$ & $\mathrm{N}$ & $\mathrm{N}$ & 11 \\
\hline Gordon & 2008 & ns & $\mathrm{N}$ & $\mathrm{Y}$ & $\mathrm{N}$ & $\mathrm{N}$ & $\mathrm{Y}$ & $\mathrm{N}$ & $\mathrm{N}$ & $\mathrm{N}$ & $\mathrm{N}$ & $\mathrm{N}$ & $\mathrm{N}$ & $\mathrm{N}$ & $\mathrm{N}$ & $\mathrm{N}$ & $\mathrm{Y}$ & $\mathrm{Y}$ & $\mathrm{N}$ & $\mathrm{Y}$ & $\mathrm{N}$ & $\mathrm{N}$ & $\mathrm{N}$ & $\mathrm{N}$ & 5 \\
\hline Hill \& MacArthur & 2006 & $\begin{array}{l}\text { St. 1: } 66 \% \\
\text { St. 2: } 48 \%\end{array}$ & $\mathrm{~N}$ & $\mathrm{Y}$ & Y & $\mathrm{Y}$ & $\mathrm{Y}$ & $\mathrm{Y}$ & $\mathrm{Y}$ & Y & $\mathrm{N}$ & $\mathrm{Y}$ & $\mathrm{N}$ & $\mathrm{N}$ & $\mathrm{N}$ & $\mathrm{Y}$ & $\mathrm{Y}$ & $\mathrm{Y}$ & $\mathrm{N}$ & Y & $\mathrm{N}$ & $\mathrm{Y}$ & $\mathrm{N}$ & $\mathrm{Y}$ & 14 \\
\hline Jeong, King \& Kim & 2007 & ns & $\mathrm{N}$ & $\mathrm{Y}$ & $\mathrm{Y}$ & $\mathrm{Y}$ & $\mathrm{Y}$ & $\mathrm{Y}$ & $\mathrm{N}$ & $\mathrm{N}$ & $\mathrm{N}$ & $\mathrm{Y}$ & $\mathrm{Y}$ & $\mathrm{Y}$ & $\mathrm{N}^{2}$ & $\mathrm{Y}$ & $\mathrm{Y}$ & $\mathrm{Y}$ & $\mathrm{N}$ & $\mathrm{Y}$ & $\mathrm{N}$ & $\mathrm{N}$ & $\mathrm{N}$ & $\mathrm{N}$ & 12 \\
\hline
\end{tabular}




\begin{tabular}{|c|c|c|c|c|c|c|c|c|c|c|c|c|c|c|c|c|c|c|c|c|c|c|c|c|c|}
\hline \multirow[b]{2}{*}{ Author } & \multirow[b]{2}{*}{ Year } & \multirow{2}{*}{\multicolumn{2}{|c|}{\begin{tabular}{l|l} 
& \\
& 1
\end{tabular}}} & \multicolumn{2}{|c|}{ Intro } & \multicolumn{9}{|c|}{ Methods } & \multicolumn{5}{|c|}{ Results } & \multicolumn{4}{|c|}{ Discussion } & \multirow[b]{2}{*}{22} & \\
\hline & & & & 2 & 3 & 4 & 5 & 6 & 7 & 8 & 9 & 10 & 11 & 12 & 13 & 14 & 15 & 16 & 17 & 18 & 19 & 20 & 21 & & \\
\hline & & 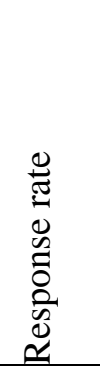 & 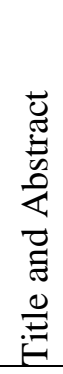 & 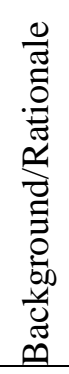 & $\frac{\sum_{0}^{0}}{\frac{0}{0}}$ & 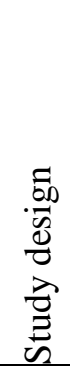 & $\stackrel{\infty}{. \Xi}$ & 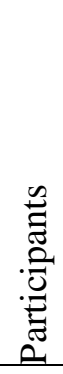 & $\frac{\frac{0}{\pi}}{\frac{\pi}{3}}$ & 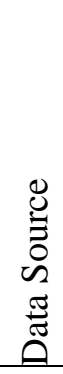 & . & 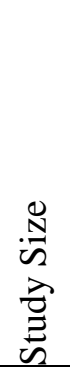 & 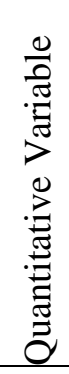 & 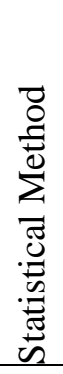 & 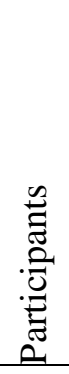 & 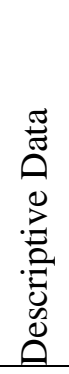 & 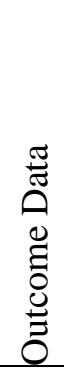 & 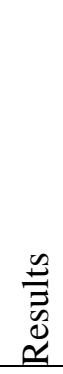 & 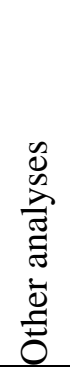 & 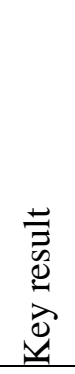 & .0. & 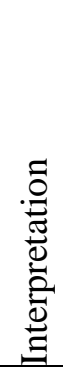 & 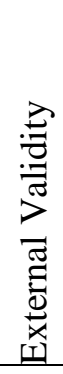 & $\stackrel{\infty}{\Xi}$ & 承 \\
\hline Loh et al. & 2002 & $\mathrm{n} / \mathrm{a}$ & $\mathrm{N}$ & $\mathrm{Y}$ & $\mathrm{Y}$ & $\mathrm{Y}$ & $\mathrm{Y}$ & $\mathrm{Y}$ & $\mathrm{N}$ & $\mathrm{Y}$ & $\mathrm{Y}$ & $\mathrm{Y}$ & $\mathrm{N}$ & $\mathrm{Y}$ & $\mathrm{Y}$ & $\mathrm{Y}$ & $\mathrm{Y}$ & $\mathrm{Y}$ & $\mathrm{N}$ & $\mathrm{Y}$ & $\mathrm{N}$ & $\mathrm{Y}$ & $\mathrm{N}$ & $\mathrm{N}$ & 15 \\
\hline Mori et al. & 2007 & $40 \%$ & $\mathrm{Y}$ & $\mathrm{Y}$ & $\mathrm{Y}$ & $\mathrm{Y}$ & $\mathrm{Y}$ & $\mathrm{Y}$ & $\mathrm{Y}$ & $\mathrm{Y}$ & $\mathrm{Y}$ & $\mathrm{Y}$ & $\mathrm{Y}$ & $\mathrm{Y}$ & $\mathrm{Y}$ & $\mathrm{Y}$ & $\mathrm{Y}$ & $\mathrm{Y}$ & $\mathrm{Y}$ & $\mathrm{Y}$ & $\mathrm{N}$ & $\mathrm{Y}$ & $\mathrm{Y}$ & $\mathrm{Y}$ & 20 \\
\hline Mueller & 2001 & $\mathrm{n} / \mathrm{a}$ & $\mathrm{N}$ & $\mathrm{Y}$ & $\mathrm{Y}$ & $\mathrm{Y}$ & $\mathrm{Y}$ & $\mathrm{Y}$ & $\mathrm{Y}$ & $\mathrm{Y}$ & $\mathrm{N}$ & $\mathrm{Y}$ & $\mathrm{Y}$ & $\mathrm{Y}$ & $\mathrm{Y}$ & $\mathrm{Y}$ & $\mathrm{Y}$ & $\mathrm{Y}$ & $\mathrm{Y}$ & $\mathrm{Y}$ & $\mathrm{Y}$ & $\mathrm{Y}$ & $\mathrm{Y}$ & $\mathrm{Y}$ & 19 \\
\hline Mueller \& Mamo & 2000 & $\mathrm{n} / \mathrm{a}$ & $\mathrm{Y}$ & $\mathrm{Y}$ & $\mathrm{Y}$ & $\mathrm{Y}$ & $\mathrm{Y}$ & $\mathrm{Y}$ & $\mathrm{Y}$ & $\mathrm{Y}$ & $\mathrm{N}$ & $Y$ & $\mathrm{Y}$ & $\mathrm{N}$ & $\mathrm{Y}$ & $Y$ & $Y$ & $\mathrm{Y}$ & $Y$ & $\mathrm{Y}$ & $\mathrm{N}$ & $Y$ & $\mathrm{~N}$ & $\mathrm{~N}$ & 17 \\
\hline Mueller \& Mamo & 2002 & $\mathrm{n} / \mathrm{a}$ & $\mathrm{N}$ & $\mathrm{Y}$ & $\mathrm{Y}$ & $\mathrm{Y}$ & $\mathrm{Y}$ & $\mathrm{Y}$ & $\mathrm{Y}$ & $\mathrm{Y}$ & $\mathrm{N}$ & $\mathrm{Y}$ & $\mathrm{Y}$ & $\mathrm{N}$ & $\mathrm{Y}$ & $\mathrm{Y}$ & $\mathrm{Y}$ & $\mathrm{Y}$ & $\mathrm{N}$ & $\mathrm{Y}$ & $\mathrm{Y}$ & $\mathrm{Y}$ & $\mathrm{N}$ & $\mathrm{N}$ & 16 \\
\hline Nagel et al. & 2010 & ns & $\mathrm{Y}$ & $\mathrm{Y}$ & $\mathrm{Y}$ & $\mathrm{Y}$ & $\mathrm{Y}$ & $\mathrm{Y}$ & $\mathrm{Y}$ & $\mathrm{Y}$ & $\mathrm{N}$ & $\mathrm{Y}$ & $\mathrm{Y}$ & $\mathrm{Y}$ & $\mathrm{Y}$ & $\mathrm{Y}$ & $\mathrm{Y}$ & $\mathrm{Y}$ & $\mathrm{Y}$ & $\mathrm{Y}$ & $\mathrm{Y}$ & $\mathrm{Y}$ & $\mathrm{Y}$ & $\mathrm{N}$ & 20 \\
\hline Rickard et al. & 2006 & $71 \%$ & $\mathrm{Y}$ & $\mathrm{Y}$ & $\mathrm{Y}$ & $\mathrm{Y}$ & $\mathrm{Y}$ & $\mathrm{Y}$ & $\mathrm{Y}$ & $\mathrm{Y}$ & $\mathrm{Y}$ & $\mathrm{Y}$ & $\mathrm{Y}$ & $\mathrm{Y}$ & $\mathrm{N}^{2}$ & $\mathrm{Y}$ & $\mathrm{Y}$ & $\mathrm{Y}$ & $Y$ & $\mathrm{Y}$ & $\mathrm{N}$ & $\mathrm{Y}$ & $\mathrm{N}$ & $\mathrm{N}$ & 18 \\
\hline Rickard et al. & 2007 & $71 \%$ & $\mathrm{Y}$ & $\mathrm{Y}$ & $\mathrm{Y}$ & $\mathrm{Y}$ & $\mathrm{Y}$ & $\mathrm{Y}$ & $\mathrm{Y}$ & $\mathrm{Y}$ & $\mathrm{Y}$ & $\mathrm{Y}$ & $\mathrm{Y}$ & $\mathrm{Y}$ & $\mathrm{Y}$ & $\mathrm{Y}$ & $\mathrm{Y}$ & $\mathrm{Y}$ & $\mathrm{Y}$ & $\mathrm{Y}$ & $\mathrm{Y}$ & $\mathrm{Y}$ & $\mathrm{Y}$ & $\mathrm{N}$ & 21 \\
\hline Rico-Villademoros et al. & 2004 & $90 \%$ & $\mathrm{~N}$ & $\mathrm{Y}$ & $\mathrm{Y}$ & $\mathrm{Y}$ & $\mathrm{Y}$ & $\mathrm{Y}$ & $\mathrm{Y}$ & $\mathrm{Y}$ & $\mathrm{Y}^{2}$ & $\mathrm{Y}$ & $\mathrm{Y}$ & $\mathrm{Y}$ & $\mathrm{N}^{4}$ & $\mathrm{Y}$ & $\mathrm{Y}$ & $\mathrm{Y}$ & $\mathrm{Y}$ & $\mathrm{Y}$ & $\mathrm{Y}$ & $\mathrm{Y}$ & $\mathrm{Y}$ & $\mathrm{Y}$ & 20 \\
\hline Roberts et al. & 2006 & $71 \%$ & $\mathrm{~N}$ & $\mathrm{Y}$ & $\mathrm{Y}$ & $\mathrm{Y}$ & $\mathrm{Y}$ & $\mathrm{Y}$ & $\mathrm{Y}$ & Y & $\mathrm{N}$ & $\mathrm{Y}$ & $\mathrm{Y}$ & $\mathrm{Y}$ & $\mathrm{Y}$ & Y & $\mathrm{Y}$ & $\mathrm{Y}$ & $\mathrm{N}$ & $\mathrm{Y}$ & $\mathrm{N}$ & Y & $\mathrm{N}$ & $\mathrm{N}$ & 16 \\
\hline Roberts et al. & $2011 \mathrm{a}$ & $54 \%$ & $\mathrm{Y}$ & $\mathrm{Y}$ & $\mathrm{Y}$ & $\mathrm{Y}$ & $\mathrm{Y}$ & $\mathrm{Y}$ & $\mathrm{Y}$ & $\mathrm{Y}$ & $\mathrm{N}$ & $\mathrm{Y}$ & $\mathrm{Y}$ & $\mathrm{Y}$ & $\mathrm{Y}$ & $\mathrm{Y}$ & $\mathrm{Y}$ & $\mathrm{Y}$ & $\mathrm{Y}$ & $\mathrm{Y}$ & $\mathrm{Y}$ & $\mathrm{Y}$ & $\mathrm{Y}$ & $\mathrm{N}$ & 20 \\
\hline
\end{tabular}




\begin{tabular}{|c|c|c|c|c|c|c|c|c|c|c|c|c|c|c|c|c|c|c|c|c|c|c|c|c|c|}
\hline \multirow[b]{2}{*}{ Author } & \multirow[b]{2}{*}{ Year } & & \multirow[b]{2}{*}{1} & \multicolumn{2}{|c|}{ Intro } & \multicolumn{9}{|c|}{ Methods } & \multicolumn{5}{|c|}{ Results } & \multicolumn{4}{|c|}{ Discussion } & \multirow[b]{2}{*}{22} & \\
\hline & & & & 2 & 3 & 4 & 5 & 6 & 7 & 8 & 9 & 10 & 11 & 12 & 13 & 14 & 15 & 16 & 17 & 18 & 19 & 20 & 21 & & \\
\hline & & 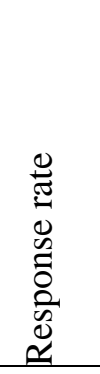 & 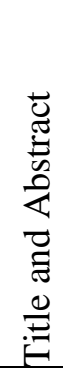 & 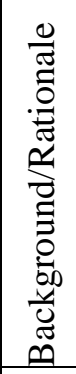 & $\begin{array}{l}\stackrel{0}{0} \\
. \frac{\tilde{U}}{0} \\
0\end{array}$ & 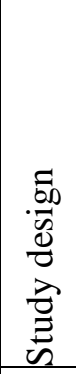 & 咆 & 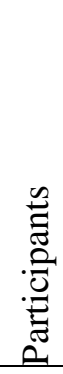 & $\frac{\frac{0}{0}}{\frac{\pi}{\pi}}$ & 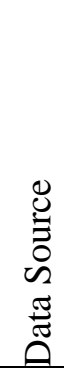 & $\frac{\tilde{t}}{\mathfrak{n}}$ & 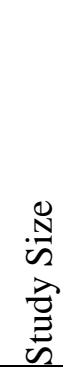 & 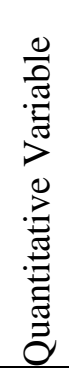 & 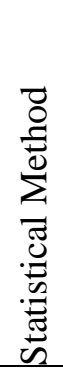 & 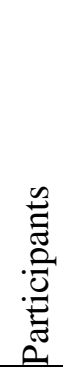 & 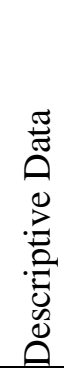 & 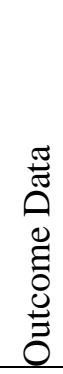 & 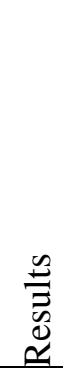 & 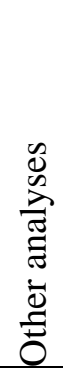 & 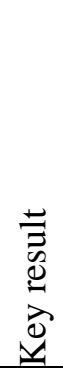 & 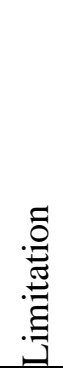 & 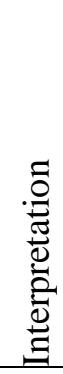 & 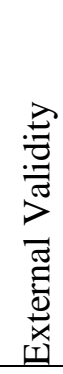 & 怘 & 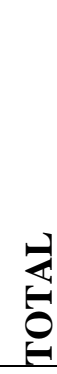 \\
\hline Roberts et al. & $2011 b$ & $54 \%$ & $\mathrm{Y}$ & $\mathrm{Y}$ & $\mathrm{Y}$ & $\mathrm{Y}$ & $\mathrm{Y}$ & $\mathrm{Y}$ & $\mathrm{Y}$ & $\mathrm{Y}$ & $\mathrm{Y}$ & $\mathrm{Y}$ & $\mathrm{Y}$ & $\mathrm{Y}$ & $\mathrm{Y}$ & $\mathrm{Y}$ & $\mathrm{Y}$ & $\mathrm{Y}$ & $\mathrm{Y}$ & $\mathrm{Y}$ & $\mathrm{Y}$ & $\mathrm{Y}$ & $\mathrm{Y}$ & $\mathrm{N}$ & 21 \\
\hline Spilsbury et al. & 2008 & $\mathrm{n} / \mathrm{a}$ & $\mathrm{N}$ & $\mathrm{Y}$ & $\mathrm{Y}$ & $\mathrm{Y}$ & $\mathrm{Y}$ & $\mathrm{Y}$ & $\mathrm{Y}$ & $\mathrm{Y}$ & $\mathrm{Y}$ & $\mathrm{Y}$ & $\mathrm{Y}$ & $Y$ & $\mathrm{Y}$ & $\mathrm{N}$ & $\mathrm{Y}$ & $\mathrm{Y}$ & $\mathrm{N}$ & $\mathrm{Y}$ & $\mathrm{N}$ & $\mathrm{Y}$ & $\mathrm{Y}$ & $\mathrm{Y}$ & 18 \\
\hline
\end{tabular}

Although the stated return rate was $83 \%, 1000$ people were invited, but only a quarter opened the email and accessed the link. Of this number, $83 \%$ participated.(Duane et al., 2007)

Did not use a validated questionnaire

Selection bias present - not overcome, but discussed

Part of questionnaire was validated; part was not

The type of methodology did not elicit a response rate eg a snowballing recruitment for focus groups 


\section{Appendix B Interview schedule - research nurses}

\section{A descriptive study about the role of the Research Nurse in \\ New Zealand tertiary level ICUs}

\begin{tabular}{|c|c|}
\hline Questions & Interviewer Prompts \\
\hline \multicolumn{2}{|c|}{ 1. Background information and employment } \\
\hline \multicolumn{2}{|c|}{ I would like to start by asking a few background questions. } \\
\hline 1.1 Do you work full or part time? & $\begin{array}{l}\text { If part-time } \\
\text { - What hours do you work? } \\
\text { - Is part-time your choice? } \\
\text { - What other work do you do? }\end{array}$ \\
\hline $\begin{array}{l}1.2 \text { Are you required to work as } \\
\text { an ICU nurse as part of your } \\
\text { employment agreement? }\end{array}$ & $\begin{array}{l}\text { - How necessary do you think it is? } \\
\text { - Would you if you didn't have to? } \\
\text { - How does it help your research position? }\end{array}$ \\
\hline $\begin{array}{l}\text { 1.3 Are you on the MECA } \\
\text { (collective contract)? }\end{array}$ & $\begin{array}{l}\text { - How are you paid if you are an on-call or have to } \\
\text { come in after hours to enrol a patient? } \\
\text { - How secure do you feel your job is? }\end{array}$ \\
\hline $\begin{array}{l}\text { 1.4 Tell me when and why you } \\
\text { became a research nurse }\end{array}$ & $\begin{array}{l}\text { Consider: } \\
\text { - Enjoy research work } \\
\text { - } \quad \text { Convenient hours }\end{array}$ \\
\hline \multicolumn{2}{|l|}{$\begin{array}{l}1.5 \text { How long have you worked in } \\
\text { your current position? }\end{array}$} \\
\hline $\begin{array}{l}\text { 1.6 What positions did you work } \\
\text { in prior to this Research Nurse } \\
\text { position? }\end{array}$ & $\begin{array}{l}\text { - Other Research } \\
\text { - ICU Nurse } \\
\text { - Other nursing } \\
\text { - If not ICU Nurse, what additional challenges do } \\
\text { you experience? }\end{array}$ \\
\hline $\begin{array}{l}\text { 1.7 When you first started as a } \\
\text { research nurse, how did you } \\
\text { learn what to do in the role? } \\
\text { How about your ICU role? }\end{array}$ & $\begin{array}{l}\text { - Formal training or study or qualifications } \\
\text { - Inservice Education } \\
\text { - Past experience } \\
\text { - Orientation - who orientated you? Was it } \\
\text { sufficient? }\end{array}$ \\
\hline $\begin{array}{l}1.8 \text { Is the work what you } \\
\text { expected when you applied for } \\
\text { the job? }\end{array}$ & - In what ways does it differ? \\
\hline $\begin{array}{l}\text { 1.9 Do you have a job } \\
\text { description? }\end{array}$ & - Do you think is is a true reflection of your role? \\
\hline $\begin{array}{l}\text { 1.10 When did you last have a } \\
\text { performance appraisal? }\end{array}$ & \\
\hline
\end{tabular}

\section{Accountability and Funding}

The next set of questions is more about the way your ICU Research department is 


\begin{tabular}{|c|c|}
\hline $\begin{array}{l}\text { 2.1 How many Research Nurses } \\
\text { work in your ICU? }\end{array}$ & - What FTE is that? \\
\hline \multicolumn{2}{|l|}{$\begin{array}{l}2.2 \text { How many medical staff are } \\
\text { Principal Investigators in your } \\
\text { ICU? }\end{array}$} \\
\hline $\begin{array}{l}\text { 2.3 Tell me about the way the } \\
\text { Research Department is } \\
\text { structured in your ICU. }\end{array}$ & $\begin{array}{l}\text { - Research Nurses } \\
\text { - Pls } \\
\text { - Managers } \\
\text { - Accountant/Finance Person } \\
\text { - Support Staff }\end{array}$ \\
\hline $\begin{array}{l}2.4 \text { Where do you fit into the } \\
\text { overall ICU structure? }\end{array}$ & $\begin{array}{l}\text { For example: } \\
\text { - } \quad \text { Are you recognised as a senior nurse? } \\
\text { - Do you attend unit-wide senior nurse meetings? }\end{array}$ \\
\hline $\begin{array}{l}2.5 \text { In your role as a Research } \\
\text { Nurse, who are you accountable } \\
\text { to for governance and } \\
\text { management issues? }\end{array}$ & $\begin{array}{l}\text { - Who is 'in charge'? } \\
\text { - Who is your line manager? } \\
\text { - How involved are they on a day to day basis? }\end{array}$ \\
\hline $\begin{array}{l}2.6 \text { How is the research } \\
\text { department in your ICU funded? } \\
\text { Is your position funded that way } \\
\text { too? }\end{array}$ & $\begin{array}{l}\text { For example: } \\
\text { - } \quad \text { Self funded through studies (trust account) } \\
\text { - } \text { Company sponsored } \\
\text { - } \quad \text { Funded through ICU budget }\end{array}$ \\
\hline $\begin{array}{l}2.7 \text { Is there a certain mix of } \\
\text { studies your ICU likes to do } \\
\text { because of financial } \\
\text { considerations? }\end{array}$ & $\begin{array}{l}\text { For example: } \\
\text { - } 1 \text { pharmaceutical sponsored study/CTG studies }\end{array}$ \\
\hline \multicolumn{2}{|c|}{$\begin{array}{l}\text { One of the recommendations that the College of Intensive Care Medicine (IC-3) has } \\
\text { made for tertiary training accredited ICUs is that your ICU should have access to "an } \\
\text { appropriate level of funding for research coordinators to support the active research } \\
\text { programme". }\end{array}$} \\
\hline $\begin{array}{l}\text { 2.8 What do you think about this } \\
\text { guideline? } \\
\text { How do you think it should be } \\
\text { funded? }\end{array}$ & \\
\hline \multicolumn{2}{|l|}{$\begin{array}{l}\text { 2.9 Who manages the finances } \\
\text { and makes financial decisions for } \\
\text { the research department within } \\
\text { your ICU? }\end{array}$} \\
\hline $\begin{array}{l}2.10 \text { How are meetings such as } \\
\text { the annual CTG in Noosa } \\
\text { funded? } \\
\text { How important do you consider it } \\
\text { is to attend these meetings to } \\
\text { help you do your job well? }\end{array}$ & $\begin{array}{l}\text { - Is it dependent on funds? } \\
\text { - } \quad \text { Funded through study grants? }\end{array}$ \\
\hline $\begin{array}{l}\text { 2.11 What do you see the role of } \\
\text { your hospital research } \\
\text { department as being? }\end{array}$ & $\begin{array}{l}\text { - How much do you pay for overheads or other } \\
\text { fees? } \\
\text { - Does the service they deliver meet your }\end{array}$ \\
\hline
\end{tabular}


expectations?

- Any other issues?

\begin{tabular}{|c|c|}
\hline \multicolumn{2}{|l|}{ 3. Research Nursing Role } \\
\hline \multicolumn{2}{|c|}{ Now I will be asking you more about your role and responsibilities as a Research Nurse } \\
\hline $\begin{array}{l}3.1 \text { What studies is your ICU } \\
\text { currently participating in? }\end{array}$ & $\begin{array}{ll}\text { For example: } \\
\text { - } \text { CHEST } \\
\text { - } \text { EPO-TBI } \\
\text { - } \text { PROWESS-Shock } \\
\text { - } \text { POLAR } \\
\text { - } \text { HEAT }\end{array}$ \\
\hline $\begin{array}{l}\text { 3.2 Tell me how it works with the } \\
\text { studies - do you all work on the } \\
\text { same studies or are you } \\
\text { responsible for individual ones? }\end{array}$ & $\begin{array}{l}\text { - Do you all have the same role in every study? } \\
\text { - Are there parts of a study that one nurse is } \\
\text { responsible for? } \\
\text { - How is the allocation worked out? } \\
\text { - Any issues? }\end{array}$ \\
\hline $\begin{array}{l}\text { 3.3 What involvement do you } \\
\text { have in nursing research? }\end{array}$ & $\begin{array}{ll}\text { - } & \text { Your own projects } \\
\text { - } & \text { Other nursing projects }\end{array}$ \\
\hline \multicolumn{2}{|c|}{ l'd like you to think about the last day you worked as a Research Nurse } \\
\hline \multicolumn{2}{|l|}{$\begin{array}{l}\text { 3.4 Tell me about what you did } \\
\text { that day and how. Think about } \\
\text { interruptions too. }\end{array}$} \\
\hline \multicolumn{2}{|l|}{$\begin{array}{l}3.5 \text { Was the day you described a } \\
\text { typical day? } \\
\text { Why? }\end{array}$} \\
\hline $\begin{array}{l}\text { 3.6 Can you tell me what sort of } \\
\text { things could happen on an } \\
\text { atypical day? }\end{array}$ & $\begin{array}{l}\text { Consider: } \\
\text { - } \quad \text { Protocol Violations } \\
\text { - } \quad \text { Serious Adverse Events } \\
\text { - } \quad \text { Assisting staff with research projects } \\
\text { - } \quad \text { Monitoring }\end{array}$ \\
\hline $\begin{array}{l}\text { 3.7 What do you think Research } \\
\text { Nurses bring to research in ICU? }\end{array}$ & $\begin{array}{l}\text { - What do you consider the most important part of } \\
\text { your role as a research nurse is? }\end{array}$ \\
\hline \multicolumn{2}{|l|}{$\begin{array}{l}\text { 3.8 How do you think research } \\
\text { nurses contribute to patient care } \\
\text { and outcomes? }\end{array}$} \\
\hline \multicolumn{2}{|c|}{$\begin{array}{l}\text { You're probably aware that in some Australian ICUs and many other clinical areas not all } \\
\text { Research Coordinators are nurses. }\end{array}$} \\
\hline $\begin{array}{l}3.9 \text { How important do you think } \\
\text { being a nurse is in your role as a } \\
\text { Research Coordinator in ICU? }\end{array}$ & - In what ways? \\
\hline \multicolumn{2}{|c|}{$\begin{array}{l}\text { A lot of the literature talks about one of the main roles of the Research Nurse as being a } \\
\text { patient advocate, which is one of our roles as a nurse. }\end{array}$} \\
\hline $\begin{array}{l}\text { 3.10 Can you tell me about a } \\
\text { situation in research when you } \\
\text { had to act as a patient advocate? }\end{array}$ & $\begin{array}{l}\text { Was there a time when you would have liked to } \\
\text { advocate or a patient (in research) but didn't feel } \\
\text { able to? }\end{array}$ \\
\hline 3.11 What skills do you think are & Consider: \\
\hline
\end{tabular}




\begin{tabular}{|c|c|}
\hline $\begin{array}{l}\text { necessary for being an ICU } \\
\text { Research Nurse? }\end{array}$ & $\begin{array}{ll}\text { - } & \text { ICU clinical skills } \\
\text { - } & \text { Research } \\
\text { - } & \text { Multi-tasking } \\
\text { - } & \text { Communication } \\
\end{array}$ \\
\hline $\begin{array}{l}3.12 \text { Have you ever been } \\
\text { involved in protocol, CRF or } \\
\text { eCRF development? } \\
\text { Or on a management committee } \\
\text { for a study? }\end{array}$ & $\begin{array}{l}\text { - What do think Research Nurses could contribute } \\
\text { in these situations? }\end{array}$ \\
\hline $\begin{array}{l}3.13 \text { What challenges do you } \\
\text { face in your role? }\end{array}$ & $\begin{array}{l}\text { Consider: } \\
\text { - Staff frustrations } \\
\text { - } \quad \text { Non-adherence to protocol } \\
\text { - } \quad \text { Short of time } \\
\text { - } \quad \text { Paperwork }\end{array}$ \\
\hline \multicolumn{2}{|c|}{$\begin{array}{l}\text { The international literature talks about one of the challenges for Research Nurses being } \\
\text { a sense of isolation they experience. I'm interested to see if this is a problem in New } \\
\text { Zealand ICUs because there are so few of us. }\end{array}$} \\
\hline $\begin{array}{l}3.14 \text { Have you ever experienced } \\
\text { a sense of isolation? }\end{array}$ & $\begin{array}{l}\text { - Professionally from other staff } \\
\text { - Geographically isolated from support network }\end{array}$ \\
\hline $\begin{array}{l}3.15 \text { If you did feel isolated, what } \\
\text { things would help or have helped } \\
\text { in the past? }\end{array}$ & \\
\hline $\begin{array}{l}3.16 \text { In what situations would you } \\
\text { assist the bedside nurse with } \\
\text { direct patient care? } \\
\text { It may or may not be research } \\
\text { related. }\end{array}$ & $\begin{array}{l}\text { Consider: } \\
\text { - Doing extra bloods when you're taking trial } \\
\text { bloods } \\
\text { - Helping with turning heavy patients } \\
\text { - Getting equipment or staff stuck at bedspace or } \\
\text { in a sideroom } \\
\text { - Do you help with short-staffing or do lunch } \\
\text { reliefs? } \\
\text { - If there was an emergency like a patient you } \\
\text { were nearby having a cardiac arrest or a patient } \\
\text { trying to extubated themselves, what would you } \\
\text { expect to contribute to the arrest situation? }\end{array}$ \\
\hline
\end{tabular}

\section{Processes around studies}

The next set of questions is about who does each aspect for studies. I'll start with the process when you are considering new studies, through to the day-to-day work involved in running studies

4a. Considering new studies

4.1 Tell me about the process in your ICU when a new trial is being considered.
- What do you take into consideration (fee being offered, whether it's cost neutral, clinical relevance, areas of interest)?

- Who is involved in the final decision?

- Who works out feasibility (budget and number of potential patients) of doing a new study? 
- Is the Research Nurse workload discussed with you?

\begin{tabular}{|c|c|}
\hline \multicolumn{2}{|c|}{ 4b. Processes when you are starting a new study } \\
\hline $\begin{array}{l}4.2 \text { Once your unit decides to do } \\
\text { a study, tell me what happens } \\
\text { then. }\end{array}$ & $\begin{array}{l}\text { Who is responsible for the following? } \\
\text { - Budget } \\
\text { - } \text { Maori approval } \\
\text { - } \text { Locality assessment } \\
\text { - Ethics application } \\
\text { - Information Sheet } \\
\text { - } \text { Contract } \\
\text { - Educates staff } \\
\text { - } \quad \text { Anything else? }\end{array}$ \\
\hline
\end{tabular}

\begin{tabular}{|c|c|}
\hline $\begin{array}{l}\text { 4c. Day-to-day Management of } \\
\text { studies }\end{array}$ & \\
\hline $\begin{array}{l}4.3 \text { Who is responsible for } \\
\text { maintaining the ongoing ethics } \\
\text { reporting in your ICU? }\end{array}$ & $\begin{array}{l}\text { - If you are the lead site, who in your unit does the } \\
\text { reports to ethics committees? } \\
\text { - Do you have a system for knowing when these } \\
\text { are due? } \\
\text { - If you're not the lead site, do you know when } \\
\text { reports are due? }\end{array}$ \\
\hline $\begin{array}{l}4.4 \text { Tell me about recruiting } \\
\text { patients for studies in your ICU }\end{array}$ & $\begin{array}{l}\text { - Whose role is it? } \\
\text { - Who identifies patients? } \\
\text { - Who does the screening log? } \\
\text { - How do you communicate about missed } \\
\text { patients? } \\
\text { - Any issues? }\end{array}$ \\
\hline $\begin{array}{l}\text { 4.5 What is your role in obtaining } \\
\text { consent for clinical trials? }\end{array}$ & $\begin{array}{l}\text { If nurse doesn't obtain consent: } \\
\text { - Who does the consent in your unit? } \\
\text { - Why don't you? } \\
\text { - Who answers questions that patients have about } \\
\text { the studies? } \\
\text { - Any issues with this? }\end{array}$ \\
\hline $\begin{array}{l}\text { 4.6 Tell me about your } \\
\text { experience using the delayed } \\
\text { consent option for any current or } \\
\text { past studies. }\end{array}$ & $\begin{array}{l}\text { - What do you think about delayed consent in the } \\
\text { ICU setting? }\end{array}$ \\
\hline $\begin{array}{l}\text { 4.7 Tell me about data collection } \\
\text { and follow-up for studies }\end{array}$ & $\begin{array}{l}\text { - Who does it? } \\
\text { - Do ever get help from other staff? e.g. with data } \\
\text { entry or phone calls } \\
\text { - Do you think it is/would be appropriate for } \\
\text { someone other than a research nurse to help with } \\
\text { this? }\end{array}$ \\
\hline 4.8 What is your role when a & - Getting ready for monitoring \\
\hline
\end{tabular}




\begin{tabular}{|l|l|}
\hline study is being monitored? & $\bullet \quad$ Any issues? \\
\hline 4.9 In what circumstances, if any & Think about: \\
do you delegate research duties & - Blood collection, study drug administration \\
to staff at the bedside? & $\bullet \quad$ Timing of patient observations \\
How do you manage this? & \\
\hline
\end{tabular}

\begin{tabular}{|c|c|}
\hline \multicolumn{2}{|l|}{ 5. Development of the Role } \\
\hline \multicolumn{2}{|c|}{$\begin{array}{l}\text { We've talked at some of our New Zealand meetings about developing the Research } \\
\text { Nurse role and getting more recognition. }\end{array}$} \\
\hline $\begin{array}{l}5.1 \text { What do you think needs to } \\
\text { happen for the role to be } \\
\text { developed? }\end{array}$ & $\begin{array}{l}\text { - } \text { Professionally across New Zealand } \\
\text { - Within your unit } \\
\text { - } \quad \text { Career Pathway }\end{array}$ \\
\hline \multicolumn{2}{|c|}{$\begin{array}{l}\text { I'm not sure if you're aware but there is a consultation paper about developing a } \\
\text { Competency Framework for Research Nurses in New Zealand. }\end{array}$} \\
\hline $\begin{array}{l}5.2 \text { Have you read it? } \\
\text { What do you think about it? }\end{array}$ & $\begin{array}{l}\text { If not: What do you think about this sort of work } \\
\text { going on? }\end{array}$ \\
\hline \multicolumn{2}{|c|}{$\begin{array}{l}\text { One of the recommendations that the ANZICS Clinical Trials Group have agreed to is } \\
\text { that there should always be a Research Coordinator on the management committee for } \\
\text { CTG-endorsed studies. }\end{array}$} \\
\hline $\begin{array}{l}5.3 \text { What do you think about this } \\
\text { recommendation? } \\
\text { What impact, if any, do you think } \\
\text { it will have on New Zealand } \\
\text { Research Nurses? }\end{array}$ & \\
\hline
\end{tabular}

\section{End of interview}

Any other comments? 


\section{Appendix C Interview schedule - principal investigators}

\section{A descriptive study about the role of the Research Nurse in}

New Zealand tertiary level ICUs

\section{Questions}

\section{Interviewer Prompts}

\section{Background information}

I would like to start by getting some background about your ICU and your work with Research Nurses.

1.1 Can I confirm that your ICU is $\quad$ (Details completed before each interview) currently participating in the following studies?

1.2 Which of these studies are you the PI for?

1.3 Can you tell me which major studies you have been involved in, and your role, in the past? 1.4 How long have you been working as a Principal Investigator for ICU studies? And have you worked with a Research Nurse all of that time?

- Role - Chief Investigator, PI, Co-investigator

- Now that you have worked with a Research Nurse, how is it different?

\begin{tabular}{|c|c|}
\hline \multicolumn{2}{|l|}{ 2. Accountability and Funding } \\
\hline \multicolumn{2}{|c|}{$\begin{array}{l}\text { The next set of questions is more about the structure and funding of your ICU Research } \\
\text { department. }\end{array}$} \\
\hline $\begin{array}{l}2.1 \text { I understand that research in } \\
\text { your ICU is ................ funded }\end{array}$ & $\begin{array}{l}\text { - How worried are you about the finances and } \\
\text { funding of research in your ICU? }\end{array}$ \\
\hline \multicolumn{2}{|l|}{$\begin{array}{l}2.2 \text { Who manages the finances } \\
\text { and makes financial decisions for } \\
\text { the research department within } \\
\text { your ICU? }\end{array}$} \\
\hline $\begin{array}{l}2.3 \text { Is there a certain mix of } \\
\text { studies your ICU likes to do } \\
\text { because of financial } \\
\text { considerations? }\end{array}$ & $\begin{array}{l}\text { - E.g. } 1 \text { pharmaceutical sponsored study/CTG } \\
\text { studies }\end{array}$ \\
\hline $\begin{array}{l}2.4 \text { What is your view about } \\
\text { funded vs non-funded research? }\end{array}$ & $\begin{array}{l}\text { - For example, would you consider taking on an } \\
\text { unfunded study if it was something that as a unit you } \\
\text { had a particular interest in? }\end{array}$ \\
\hline $\begin{array}{l}2.5 \text { Tell me about the way your } \\
\text { ICU Research Department is } \\
\text { structured }\end{array}$ & $\begin{array}{l}\text { - } \text { Pls } \\
\text { - Research Nurses } \\
\text { - Managers } \\
\text { - Accountant/Finance Person } \\
\text { - Support Staff } \\
\text { - Who is 'in charge'? }\end{array}$ \\
\hline 2.6 Where would you say & For example: \\
\hline
\end{tabular}




\begin{tabular}{|l|l|}
\hline $\begin{array}{l}\text { Research Nurses fit into the } \\
\text { overall ICU structure? }\end{array}$ & $\begin{array}{l}\text { - Are they recognised as senior nurses? } \\
\text { - Do they attend unit-wide senior nurse meetings? }\end{array}$ \\
\hline $\begin{array}{l}\begin{array}{l}\text { is How important do you think it } \\
\text { is for Rearch Nurses to attend } \\
\text { meetings such as the annual } \\
\text { CTG in Noosa? }\end{array} \\
\text { How are these meetings funded? }\end{array}$ & - E.g. Trust account or study grants \\
\hline $\begin{array}{l}\text { 2.8 What do you see the role of } \\
\text { your hospital research } \\
\text { department as being? }\end{array}$ & $\begin{array}{l}\text { - How much do you pay for overheads or other } \\
\text { fees? } \\
\text { - Does the service they deliver meet your } \\
\text { expectations? } \\
\text { - Any other issues? }\end{array}$ \\
\hline
\end{tabular}

\begin{tabular}{|c|c|}
\hline \multicolumn{2}{|l|}{ 3. Research Nursing Role } \\
\hline \multicolumn{2}{|c|}{$\begin{array}{l}\text { Now l'd like to ask you about you more about the role and responsibilities of the } \\
\text { Research Nurse. }\end{array}$} \\
\hline $\begin{array}{l}3.1 \text { Tell me about the role of the } \\
\text { Research Nurse in relation to } \\
\text { your role as Principal } \\
\text { Investigator. }\end{array}$ & $\begin{array}{l}\text { - In what things do you consider they are } \\
\text { accountable to you? } \\
\text { - Who is their line manager? } \\
\text { - Do you work as a team on particular studies? }\end{array}$ \\
\hline $\begin{array}{l}3.2 \text { What do you consider their } \\
\text { role and responsibilities in } \\
\text { research to be? }\end{array}$ & $\begin{array}{l}\text { Consider: } \\
\text { - Compared to the role of the PI } \\
\text { - Day to day management of studies } \\
\text { - Does the PI have overall responsibility for } \\
\text { studies? }\end{array}$ \\
\hline $\begin{array}{l}\text { 3.3 Do you know how the } \\
\text { Research Nurses are orientated } \\
\text { to the role within ICU? Do you } \\
\text { assist in this? }\end{array}$ & \\
\hline $\begin{array}{l}3.4 \text { What sort of occupational } \\
\text { background helps them to fulfil } \\
\text { the role expectations of an ICU } \\
\text { Research Nurse? }\end{array}$ & \\
\hline $\begin{array}{l}\text { 3.5 Do you think Research } \\
\text { Nurses should also do bedside } \\
\text { nursing in ICU on a regular } \\
\text { basis? }\end{array}$ & $\begin{array}{l}\text { - What do you think this brings to their role as } \\
\text { Research Nurses? }\end{array}$ \\
\hline $\begin{array}{l}3.6 \text { If the unit was short staffed, } \\
\text { would you expect the Research } \\
\text { Nurse to change from research } \\
\text { duties to clinical duties? }\end{array}$ & - Or help out with lunch reliefs or side rooms? \\
\hline $\begin{array}{l}3.7 \text { If there was an emergency } \\
\text { like a patient having a cardiac } \\
\text { arrest or a patient was trying to } \\
\text { extubated themselves and the } \\
\text { Research Nurse was nearby, }\end{array}$ & \\
\hline
\end{tabular}


what would you expect the Research Nurse to do in this situation?

\section{Processes around studies}

The next set of questions is to help me get an understanding of who does the various aspects required for clinical trials. I'll start with the process when you are considering new studies, through to the day-to-day work involved in running studies

\section{4a. Considering new studies}

4.1 Tell me about the process in your ICU when a new trial is being considered.
- What do you take into consideration (per patient fee, cost neutral, clinical relevance, areas of interest)?

- Who is involved in the final decision?

- Who works out feasibility (budget and number of potential patients) of doing a new study?

- Is the potential workload discussed with the Research Nurse?

\section{4b. Processes when you are starting a new study}

4.2 Once your unit decides to do a study, tell me what happens then.
Who is responsible for the following?

- Budget

- Maori approval

- Locality assessment

- Ethics application

- Information Sheet

- Contract

- Educates staff (doctors and nurses)

- Anything else?

4c. Day-to-day Management of studies

4.3 Who is responsible for maintaining ongoing ethics reporting in your ICU?

4.4 Tell me about recruiting patients for studies in your ICU

\subsection{What is the Research Nurse's} role in obtaining consent for clinical trials?

4.6 Tell me about your experience using the delayed consent option for any current or past studies.

4.7 Tell me about data collection

- Who does it?

- If you are the lead site, who in your unit does the reports to ethics committees?

- Whose role is it?

- Who identifies patients?

- Who does the screening log?

- How do you communicate about missed patients?

- Any issues?

- Are there any circumstances where you do/don't think it's appropriate for them to obtain consent?

- Any issues with this?

- What do you think about delayed consent in the ICU setting? 


\begin{tabular}{|l|l|}
\hline and follow-up for studies & $\begin{array}{l}\text { - Do you think it would be appropriate for } \\
\text { someone other than a research nurse to help with } \\
\text { this? }\end{array}$ \\
\hline $\begin{array}{l}\text { 4.8 What happens when you } \\
\text { have protocol violations or } \\
\text { serious adverse events occur? }\end{array}$ & $\begin{array}{l}\text { - Who tends to notice them? } \\
\text { - Who reports them? } \\
\text { - Who follows up with staff involved? (Doctor or } \\
\text { nurses) }\end{array}$ \\
\hline $\begin{array}{l}\text { 4.9 Who's responsibility is it to } \\
\text { prepare for monitoring visits? }\end{array}$ & - Any issues? \\
\hline
\end{tabular}

\begin{tabular}{|c|c|}
\hline \multicolumn{2}{|c|}{ 5. Development of the Research Nurse Role } \\
\hline \multicolumn{2}{|c|}{$\begin{array}{l}\text { One of the recommendations that the College of Intensive Care Medicine (IC-3) has } \\
\text { made for tertiary training accredited ICUs is that your ICU should have access to "an } \\
\text { appropriate level of funding for research coordinators to support the active research } \\
\text { programme". }\end{array}$} \\
\hline $\begin{array}{l}5.1 \text { What do you think of this } \\
\text { guideline? } \\
\text { How would you like to see it } \\
\text { funded? }\end{array}$ & \\
\hline \multicolumn{2}{|c|}{$\begin{array}{l}\text { One of the recommendations that the ANZICS Clinical Trials Group have agreed to is } \\
\text { that there should always be a research coordinator on the management committee for } \\
\text { CTG-endorsed studies. }\end{array}$} \\
\hline $\begin{array}{l}5.2 \text { What do you think about this } \\
\text { recommendation? } \\
\text { What impact, if any, do you think } \\
\text { it will have in New Zealand? }\end{array}$ & \\
\hline $\begin{array}{l}5.3 \text { What do you think Research } \\
\text { Nurses bring to research in ICU? }\end{array}$ & \\
\hline $\begin{array}{l}5.4 \text { How do you think research } \\
\text { nurses contribute to patient care } \\
\text { and outcomes? }\end{array}$ & \\
\hline
\end{tabular}

\section{End of interview}

Any other comments? 


\section{Appendix D Interview schedule - nurse managers \\ A descriptive study about the role of the Research Nurse in \\ New Zealand tertiary level ICUs}

\begin{tabular}{|c|c|}
\hline Questions & Interviewer Prompts \\
\hline \multicolumn{2}{|l|}{ 1. Background information } \\
\hline \multicolumn{2}{|c|}{$\begin{array}{l}\text { I would like to start by getting some background about the research work your ICU is } \\
\text { participating in, and the overall structure of research in your ICU. }\end{array}$} \\
\hline $\begin{array}{l}1.1 \text { Can I confirm that your ICU is } \\
\text { currently participating in the } \\
\text { following studies? }\end{array}$ & (Details completed before each interview) \\
\hline $\begin{array}{l}1.2 \text { Is there any other research } \\
\text { work going on within the ICU at } \\
\text { the moment? } \\
\text { For example: Nursing research } \\
\text { Investigator-led research studies }\end{array}$ & $\begin{array}{l}\text { - Are research nurses involved in this sort of } \\
\text { work? } \\
\text { - In a support or advisory role? }\end{array}$ \\
\hline $\begin{array}{l}\text { 1.3 Tell me about the way your } \\
\text { ICU Research Department is } \\
\text { structured. }\end{array}$ & $\begin{array}{l}\text { - Research Nurses } \\
\text { - Pls } \\
\text { - Managers } \\
\text { - Accountant/Finance Person } \\
\text { - Support Staff }\end{array}$ \\
\hline $\begin{array}{l}\text { 1.4 Where would you say the } \\
\text { Research Nurses fit into the } \\
\text { overall ICU structure? }\end{array}$ & $\begin{array}{l}\text { For example: } \\
\text { - Are they recognised as senior nurses? } \\
\text { - Do they attend unit-wide senior nurse meetings? } \\
\text { - Is the research department considered part of } \\
\text { ICU or is it a separate department? }\end{array}$ \\
\hline $\begin{array}{l}1.5 \text { What is your role in relation to } \\
\text { the Research Nurses? }\end{array}$ & $\begin{array}{l}\text { - In what things do you consider they are } \\
\text { accountable to you? } \\
\text { - Are you their line manager? } \\
\text { - Who does their performance appraisals? }\end{array}$ \\
\hline $\begin{array}{l}1.6 \text { Who manages the research } \\
\text { that is done in your unit on a day- } \\
\text { to-day basis? }\end{array}$ & $\begin{array}{l}\text { - All the aspects involved with running clinical } \\
\text { trials }\end{array}$ \\
\hline
\end{tabular}

\begin{tabular}{|c|c|}
\hline \multicolumn{2}{|l|}{ 2. Research Nursing Role } \\
\hline \multicolumn{2}{|c|}{$\begin{array}{l}\text { Now l'd like to ask you about you more about the role and responsibilities of the } \\
\text { Research Nurse. }\end{array}$} \\
\hline You have multiple studies going $\mathrm{c}$ & at any one time. \\
\hline $\begin{array}{l}2.1 \text { How is it decided which } \\
\text { research nurse is responsible for } \\
\text { each study? }\end{array}$ & $\begin{array}{l}\text { For example: } \\
\text { - Are they each responsible for a study or do they } \\
\text { all have the same role in every study? }\end{array}$ \\
\hline
\end{tabular}




\begin{tabular}{|c|c|}
\hline $\begin{array}{l}2.2 \text { What do you know about how } \\
\text { the research nurse is orientated } \\
\text { into the role? }\end{array}$ & \\
\hline $\begin{array}{l}2.3 \text { Tell me about what you } \\
\text { consider to be the role and } \\
\text { responsibilities of the Research } \\
\text { Nurse. }\end{array}$ & $\begin{array}{l}\text { Consider: } \\
\text { - } \quad \text { Day to day management of trials } \\
\text { - } \quad \text { Compared to PI }\end{array}$ \\
\hline $\begin{array}{l}2.4 \text { What sort of occupational } \\
\text { background helps them to fulfil } \\
\text { the role expectations of an ICU } \\
\text { Research Nurse? }\end{array}$ & \\
\hline $\begin{array}{l}2.5 \text { In what situations would you } \\
\text { expect the Research Nurse to } \\
\text { assist the bedside nurse with } \\
\text { direct patient care? } \\
\text { It may or may not be research } \\
\text { related. }\end{array}$ & $\begin{array}{l}\text { Consider: } \\
\text { - Helping with turning heavy patients } \\
\text { - Getting equipment or staff stuck at bedspace or } \\
\text { in a sideroom } \\
\text { - Doing routine bloods when they're taking trial } \\
\text { bloods? }\end{array}$ \\
\hline $\begin{array}{l}\text { 2.6 Do you think Research } \\
\text { Nurses should also do bedside } \\
\text { nursing in ICU on a regular } \\
\text { basis? }\end{array}$ & - What do you think this brings to their role? \\
\hline $\begin{array}{l}2.7 \text { If the unit was short staffed, } \\
\text { would you expect the Research } \\
\text { Nurse to change from research } \\
\text { duties to clinical duties? }\end{array}$ & - Or help out with lunch reliefs or side rooms? \\
\hline $\begin{array}{l}2.8 \text { If there was an emergency } \\
\text { like a patient having a cardiac } \\
\text { arrest or a patient was trying to } \\
\text { extubated themselves and the } \\
\text { Research Nurse was nearby, } \\
\text { what would you expect the } \\
\text { Research Nurse to do in this } \\
\text { situation? }\end{array}$ & \\
\hline $\begin{array}{l}2.9 \text { What do you think Research } \\
\text { Nurses bring to research in ICU? }\end{array}$ & \\
\hline
\end{tabular}

\section{Accountability and Funding}

The next set of questions is more about the funding of your ICU Research department.

3.1 I understand that research in $\bullet$ How worried are you about the finances and your ICU is funded funding of research in your ICU?

3.2 Who manages the finances and makes financial decisions for the research department within your ICU?

3.3 Is there a certain mix of studies your ICU likes to do because of financial

- For example: 1 pharmaceutical sponsored study/CTG studies

- Is the 'per patient' fee a major factor when you 


\begin{tabular}{|c|c|}
\hline considerations? & are considering a new study? \\
\hline $\begin{array}{l}3.4 \text { What is your view about } \\
\text { funded vs non-funded research? }\end{array}$ & $\begin{array}{l}\text { - For example, would you consider taking on an } \\
\text { unfunded study if it was something that as a unit you } \\
\text { had a particular interest in? }\end{array}$ \\
\hline \multicolumn{2}{|c|}{$\begin{array}{l}\text { One of the recommendations that the College of Intensive Care Medicine (IC-3) has } \\
\text { made for tertiary training accredited ICUs is that your ICU should have access to "an } \\
\text { appropriate level of funding for research coordinators to support the active research } \\
\text { programme". }\end{array}$} \\
\hline $\begin{array}{l}\text { 3.5 What do you think of this } \\
\text { guideline? } \\
\text { How do you think it should be } \\
\text { funded? }\end{array}$ & \\
\hline $\begin{array}{l}3.6 \text { How important do you think it } \\
\text { is for Research Nurses to attend } \\
\text { meetings such as the annual } \\
\text { CTG meeting in Noosa, to help } \\
\text { them do their job well? } \\
\text { How are meetings like this } \\
\text { funded? }\end{array}$ & $\begin{array}{l}\text { - Is it dependent on funds? } \\
\text { - Funded through study grants }\end{array}$ \\
\hline $\begin{array}{l}\text { 3.7 What do you see the role of } \\
\text { your hospital research } \\
\text { department as being? }\end{array}$ & $\begin{array}{l}\text { - How much do you pay for overheads or other } \\
\text { fees? } \\
\text { - Does the service they deliver meet your } \\
\text { expectations? } \\
\text { - Any other issues? }\end{array}$ \\
\hline
\end{tabular}

\section{Processes around studies}

The next set of questions is to help me get an understanding of who does the various aspects required for clinical trials. You may or may not know the answer to some of the questions as they may be managed at the clinical level. I'll start with the process when you are considering new studies, through to the day-to-day work involved in running studies.

4a. Considering new studies

4.1 Tell me about the process in your ICU when a new trial is being considered.
- What do you take into consideration (per patient fee, cost neutral, clinical relevance, areas of interest)

- Who is involved in the final decision?

- Who works out feasibility (budget and number of potential patients) of doing a new study?

- Is the potential workload discussed with the Research Nurse?

4b. Processes when you are starting a new study

4.2 Once your unit decides to do a study, tell me what happens then.
Who is responsible for the following?

- Budget

- Maori approval

- Locality assessment 


\begin{tabular}{|c|c|}
\hline & $\begin{array}{l}\text { - Ethics application } \\
\text { - Information Sheet } \\
\text { - } \text { Contract } \\
\text { - Educates staff (doctors and nurses) } \\
\text { - Anything else? }\end{array}$ \\
\hline \multicolumn{2}{|l|}{$\begin{array}{l}\text { 4c. Day-to-day Management of } \\
\text { studies }\end{array}$} \\
\hline $\begin{array}{l}4.3 \text { Who is responsible for } \\
\text { maintaining ongoing ethics } \\
\text { reporting in your ICU? }\end{array}$ & $\begin{array}{l}\text { - If you are the lead site, who in your unit does the } \\
\text { reports to ethics committees? } \\
\text { - If you're not the lead site, who would know when } \\
\text { reports are due? }\end{array}$ \\
\hline $\begin{array}{l}\text { 4.4 Tell me about recruiting } \\
\text { patients for studies in your ICU }\end{array}$ & $\begin{array}{l}\text { - Whose role is it? } \\
\text { - Who identifies patients? } \\
\text { - Who does the screening log? } \\
\text { - How do you communicate about missed } \\
\text { patients? } \\
\text { - Any issues? }\end{array}$ \\
\hline $\begin{array}{l}4.5 \text { What is the Research Nurse's } \\
\text { role in obtaining consent for } \\
\text { clinical trials? }\end{array}$ & $\begin{array}{l}\text { - Are there any circumstances where you do/don't } \\
\text { think it's appropriate for them to obtain consent? } \\
\text { - Any issues with this? }\end{array}$ \\
\hline $\begin{array}{l}\text { 4.6 Tell me about your } \\
\text { experience using the delayed } \\
\text { consent option for any current or } \\
\text { past studies. }\end{array}$ & $\begin{array}{l}\text { - What do you think about delayed consent in the } \\
\text { ICU setting? }\end{array}$ \\
\hline $\begin{array}{l}\text { 4.7 Tell me about data collection } \\
\text { and follow-up for studies }\end{array}$ & $\begin{array}{l}\text { - Who does it? } \\
\text { - Do you think it would be appropriate for } \\
\text { someone other than a research nurse to help with } \\
\text { this? }\end{array}$ \\
\hline $\begin{array}{l}\text { 4.9 What happens when you } \\
\text { have protocol violations or } \\
\text { serious adverse events occur? }\end{array}$ & $\begin{array}{l}\text { - Who tends to notice them? } \\
\text { - Who reports them? } \\
\text { - Who follows up with staff involved? (Doctor or } \\
\text { nurses) }\end{array}$ \\
\hline
\end{tabular}

\section{Development of the Role}

5.1 What do you think needs to happen for the Research Nurse role to be developed?

- Professionally across New Zealand

- Within your unit

- Career Pathway

I'm not sure if you're aware but there is a consultation paper about developing a Competency Framework for Research Nurses in New Zealand.

\begin{tabular}{l|l}
\hline 5.2 Have you read it? & If not: What do you think about this sort of work
\end{tabular} What do you think about it? going on?

One of the recommendations that the ANZICS Clinical Trials Group have agreed to is that there should always be a research coordinator on the management committee for CTG-endorsed studies.

5.3 What do you think about this 
recommendation?

What impact, if any, do you think

it will have in New Zealand?

5.4 What do you think Research

Nurses bring to research in ICU?

5.5 How do you think research nurses contribute to patient care and outcomes?

6. End of interview

Any other comments? 


\section{Appendix E Information sheet for research nurses}

VICTORIA UNIVERSITY OF WELLINGTON

Te Whare Wananga o te Upoko o te Ika a Maui

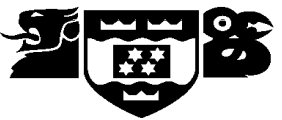

\section{Research Nurse Information Sheet}

\section{A descriptive study about the role of the Research Nurse in New Zealand tertiary level Intensive Care Units}

My name is Diane Mackle. I have worked at Wellington Hospital Intensive Care Unit (ICU) since 1996, and have been a Research Nurse there since 2007. I am currently undertaking my Master of Nursing at Victoria University of Wellington Graduate School of Nursing, Midwifery and Health. As part of this, I am completing a thesis about the role and responsibilities of the Research Nurse in New Zealand (NZ) tertiary level ICUs.

You have been invited to participate in this study because you are currently working as a Research Nurse in a tertiary-level ICU.

Ethics approval for the study has been granted by the Victoria University of Wellington Human Ethics Committee.

\section{Background}

Research Nurses, also known as Research Coordinators have been employed in NZ ICUs since approximately 1995. Research studies in NZ ICUs are increasing, and it is likely that more research nurses will be employed in the future. This study will add to the body of knowledge about research nursing, and will potentially add to the overall quality of future research. It will inform Unit Management, who may be considering employing research nurses.

Over time, the Research Nurse role has evolved in an ad hoc manner within individual ICUs. There is little literature about the role and responsibilities of the research nurse, and their place in the ICU structure. International literature has focussed on the research nurse role only from the perspective of the research nurse. This study will analyse their role and responsibilities from the perspective of Research Nurses, Principal Investigators and Unit Managers. This study is a descriptive study, using semi-structured interviews.

\section{What would participating involve?}

Participating in this research would involve being interviewed in person by the Principal Investigator about the role and responsibilities of the Research Nurse, and the structure of research undertaken within your ICU. This interview will be semistructured, take approximately $1-1 \frac{1}{2}$ hours and will be digitally recorded with your 
consent. The interviews will be conducted at a mutually agreeable time and place in March-April, 2011.

A professional transcriber will be employed and be subject to a transcriber agreement. You will be able to view your own transcript if requested, and make amendments. The data will be analysed using content and thematic analysis.

Tertiary level ICUs are commonly known within the ICU community, as are the research staff. Findings will be aggregated by theme, and quotations will be reported by research role and number. Attention will be given in the presentation of results to remove all identifiable language patterns or content data. While every effort will be made to maintain confidentiality, there is the potential for identifiability of who has participated in the study.

\section{Who will have access to the recordings, transcripts and field notes?}

The only people who will hear the recordings or see the transcripts and field notes will be the Principal Investigator, my research supervisor, and the transcriber. The transcripts and recordings will be kept in a locked cupboard until 2 years after the completion of the thesis. After this time they will be destroyed.

\section{Publication}

In addition to my thesis, it is my intention to publish the findings of this study in a New Zealand nursing journal and present at ICU and Research conferences and forums.

If you have any questions about the study, please contact:

Diane Mackle (Principal Investigator)

Specialty Nurse - Research

Intensive Care Unit

Wellington Regional Hospital

Private Bag

Wellington South

Email: $x x x x x x x x x x x x x x x x x x x x x x x x x$

Phone $x x x x x x$ xxx

Dr Katherine Nelson (Supervisor)

Senior Lecturer

Graduate School of Nursing, Midwifery \& Health

Victoria University of Wellington

P.O. Box 7625

Wellington 6242

Email: $x x x x x x x x x x x x x x x x x x x x x x x x$

Phone $x x$ xxx $x x x x$ 


\section{Appendix F Consent form for all participants}

VICTORIA UNIVERSITY OF WELLINGTON

Te Whare Wananga o te Upoko o te Ika a Maui

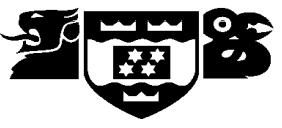

\section{CONSENT FORM}

\section{A descriptive study about the role of the Research Nurse in New Zealand tertiary level ICUs}

I have been provided with adequate information relating to the nature and objectives of this research project. I have understood that information, and have been given the opportunity to seek further clarification or explanations.

I understand that the information I provide and the digital recording of the interview will be kept confidential to the interviewer and her supervisor.

I understand that what I say in the interview will be confidential. Every effort will be made to protect my confidentiality and that of the ICU I work in. However, given the small size of the ICU research community, it is possible that I may be identifiable as a participant.

I understand that I do not have to answer any questions which I am not happy with or think could potentially identify the ICU I work in or me.

I agree to be interviewed for the above study and for the interview to be digitally recorded.

I am aware that I can withdraw from the study up to 2 weeks after the interview or after receiving the transcript. If I withdraw, the digital recording and transcript will be destroyed.

On completion of the project I understand that Diane Mackle will contact me and inform me of where I can access a copy of the completed thesis, should I wish to read it.

I would like the opportunity to review the transcript of my interview.

YES/NO

I would like a summary of the results of this study.

YES/NO

Name of Participant

Address for transcript/results

Signature of Participant

Date 
Appendix G

\author{
Information sheet for principal investigators and nurse \\ managers \\ VICTORIA UNIVERSITY OF WELLINGTON \\ Te Whare Wananga o te Upoko o te Ika a Maui

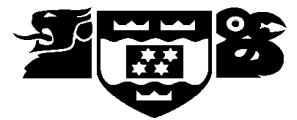

\section{Principal Investigator and Unit Manager Information sheet}

\title{
A descriptive study about the role of the Research Nurse in New Zealand tertiary level Intensive Care Units
}

My name is Diane Mackle. I have worked at Wellington Hospital Intensive Care Unit (ICU) since 1996, and have been a Research Nurse there since 2007. I am currently undertaking my Master of Nursing at Victoria University of Wellington Graduate School of Nursing, Midwifery and Health. As part of this, I am completing a thesis about the role and responsibilities of the Research Nurse in New Zealand (NZ) tertiary level ICUs.

You have been invited to participate in this study because you are currently working as Principal Investigator (or have recently) for a research study or are a Unit Manager responsible for a Research Nurse in a tertiary-level ICU.

Ethics approval for the study has been granted by the Victoria University of Wellington Human Ethics Committee.

\section{Background}

Research Nurses, also known as Research Coordinators have been employed in NZ ICUs since approximately 1995 . Research studies in NZ ICUs are increasing, and it is likely that more research nurses will be employed in the future. This study will add to the body of knowledge about research nursing, and will potentially add to the overall quality of future research. It will inform Unit Management, who may be considering employing research nurses.

Over time, the Research Nurse role has evolved in an ad hoc manner within individual ICUs. There is little literature about the role and responsibilities of the research nurse, and their place in the ICU structure. International literature has focussed on the research nurse role only from the perspective of the Research Nurse. This study will analyse their role and responsibilities from the perspective of Research Nurses, Principal Investigators and Unit Managers. This study is a descriptive study, using semi-structured interviews.

\section{What would participating involve?}

Participating in this research would involve being interviewed in person by the Principal Investigator about the role and responsibilities of the Research Nurse, and the structure of research undertaken within your ICU. This interview will be semi- 
structured, take approximately 1 hour and will be digitally recorded with your consent. The interviews will be conducted at a mutually agreeable time and place in March-April, 2011.

A professional transcriber will be employed and be subject to a transcriber agreement. You will be able to view your own transcript if requested, and make amendments. The data will be analysed using content and thematic analysis.

Tertiary level ICUs are commonly known within the ICU community, as are the research staff. Findings will be aggregated by theme, and quotations will be reported by research role and number. Attention will be given in the presentation of results to remove all identifiable language patterns or content data. While every effort will be made to maintain confidentiality, there is the potential for identifiability of who has participated in the study.

\section{Who will have access to the recordings, transcripts and field notes?}

The only people who will hear the recordings or see the transcripts and field notes will be the Principal Investigator, my research supervisor, and the transcriber. The transcripts and recordings will be kept in a locked cupboard until 2 years after the completion of the thesis. After this time they will be destroyed.

\section{Publication}

In addition to my thesis, it is my intention to publish the findings of this study in a New Zealand nursing journal and present at ICU and Research conferences and forums.

If you have any questions about the study, please contact:

Diane Mackle (Principal Investigator)

Specialty Nurse - Research

Intensive Care Unit

Wellington Regional Hospital

Private Bag

Wellington South

Email: $x x x x x x x x x x x x x x x x x x x x x x x x$

Phone $x x x x x x$ xxxx

Dr Katherine Nelson (Supervisor)

Senior Lecturer

Graduate School of Nursing, Midwifery \& Health

Victoria University of Wellington

P.O. Box 7625

Wellington 6242

Email: $x x x x x x x x x x x x x x x x x x x x x x x x x x$

Phone $x x x x x x x x x$

Wellington 6242 


\title{
Appendix H Transcriber agreement
}

\author{
VICTORIA UNIVERSITY OF WELLINGTON \\ Te Whare Wananga o te Upoko o te Ika a Maui

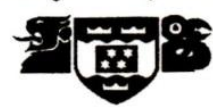

\section{Confidentiality Agreement for Transcriber of a Research study, titled:}

\section{A descriptive study about the role of the Research Nurse in New} Zealand tertiary-level Intensive Care Units

\section{Researcher: Diane Mackle}

I have read the information sheet outlining this study. I have discussed with the researcher the nature of the research and have had any questions that I have had answered to my satisfaction. My role as the research transcriber has been outlined to me by the researcher.

At all times the research information (digital recordings and transcripts) will be inaccessible to other persons. The researcher has assured me that she will debrief me following transcribing to address any issues that transcribing bring up for me.

Most importantly, I understand and agree to keep the information I hear and type in the course of transcribing confidential to the researcher and myself.

Full Name: brende watsan

Researcher's Signature:

$$
\text { diravere. }
$$

Date:

$$
\text { sh June } 2011
$$




\section{Appendix I Ethics approval}

\section{MEMORANDUM}
Phone $\quad 0-4-4635676$
Fax 0-4-4635209
Email Allison.kirkman@vuw.ac.nz

\begin{tabular}{l|l}
\hline TO & Diane Mackle \\
\hline COPY TO & Kathy Nelson \\
\hline FROM & Dr Allison Kirkman, Convener, Human Ethics Committee \\
\hline
\end{tabular}

\begin{tabular}{l|l}
\hline DATE & 25 March 2011 \\
\hline PAGES & 1 \\
\hline
\end{tabular}

\begin{tabular}{l|l}
\hline SUBJECT & $\begin{array}{l}\text { Ethics Approval: 18345 The Role of the Research Nurse in } \\
\text { Level } 3 \text { Intensive Care Units in New Zealand - a descriptive } \\
\text { study }\end{array}$ \\
\hline
\end{tabular}

Thank you for your application for ethical approval, which has now been considered by the Standing Committee of the Human Ethics Committee.

Your application has been approved from the above date and this approval continues until 31 July 2012. If your data collection is not completed by this date you should apply to the Human Ethics Committee for an extension to this approval.

Best wishes with the research.

Allison Kirkman

Human Ethics Committee 


\section{Reference list}

Ahern, J. A., Kruger, D. F., Gatcomb, P. M., Petit, W. A., \& Tamborlane, W. V. (1989). The diabetes control and complication trial (DCCT): The trial coordinator perpective. Diabetes Educator, 15(3), 236 - 241.

Anderson, G. (2008). Ethical preparedness and performance of gene therapy study coordinators. Nursing Ethics, 15(2), 207-221.

Australian and New Zealand Intensive Care Society. (2010). 'About us' webpage. Retrieved 30 November, 2010, from http://www.anzics.com.au/about-us

Australian and New Zealand Intensive Care Society Centre for Outcome and Resource Evaluation Database. (2011). New Zealand ICU Data.

Australian and New Zealand Intensive Care Society Clinical Trials Group. (2008).

Competing Studies Policy. CTG14a. Retrieved 26 April, 2012, from

http://www.anzics.com.au/ctg/ctgdocuments

Australian and New Zealand Intensive Care Society Clinical Trials Group. (2010a). CTG website. Retrieved 30 November, 2010, from http://www.anzics.com.au/clinicaltrials-group

Australian and New Zealand Intensive Care Society Clinical Trials Group. (2010b). Guiding principles for investigators wanting to incorporate research projects into the ANZICS Clinical Trials Group point prevalence program. (pp.1-4). Retrieved 14 June, 2012 from http://www.anzics.com.au/images/ctg/ctg16a2010011110_ppp_guiding_principles_f or_investigators.pdf

Australian and New Zealand Intensive Care Society Clinical Trials Group. (2011a). Activity Report 2010 - 2011. Carlton South, Australia.

Australian and New Zealand Intensive Care Society Clinical Trials Group. (2011b). Coenrolment Policy. C19a. Retrieved 26 April, 2012, from http://www.anzics.com.au/ctg/ctgdocuments

Australian and New Zealand Intensive Care Society Clinical Trials Group. (2011c). Terms of reference (Version 150811 ed., pp.1-20): ANZICS Clinical Trials Group.

Baumbusch, J. (2010). Semi-structured interviewing in practice-close research. Journal for Specialists in Pediatric Nursing, 15(3), 255-258. doi: 10.1111/j.17446155.2010.00243.x

Becze, E. (2010). Defining the role of the clinical research nurse. ONS Connect, 25(5), 1213.

Bell, J. (2009). Towards clarification of the role of research nurses in New Zealand: A literature review. Nursing Praxis in New Zealand, 25(1), 4-16.

Bevans, M., Hastings, C., Wehrlen, L., Cusack, G., Matlock, A. M., Miller-Davis, C., . . Wallen, G. R. (2011). Defining clinical research nursing practice: Results of a role 
delineation study. Clinical and Translational Science, 4(6), 421-427. doi: 10.1111/j.1752-8062.2011.00365.x

Bird, J., \& Kirshbaum, M. (2005). Towards a framework of advanced nursing practice for the clinical research nurse in cancer care. Clinical Effectiveness in Nursing, 9, 161171.

Bishop, R. (2005). Freeing ourselves from neocolonial domination in research. In N.K. Denzin \& Y. S. Lincoln (Eds.), The SAGE handbook of qualitative research (3rd ed.). Thousand Oaks, CA.: Sage.

Braun, V., \& Clarke, V. (2006). Using thematic analysis in psychology. Qualitative Research in Psycology, 3, 77 - 101.

Catania, G., Poir Ã “, I., Dozin, B., Bernardi, M., \& Boni, L. (2008). Validating a measure to delineate the clinical trials nursing role in Italy. Cancer Nursing, 31(5), E11.

Catania, G., Poirè, I., Bernardi, M., Bono, L., Cardinale, F., \& Dozin, B. (2012). The role of the clinical trial nurse in Italy. European Journal of Oncology Nursing, 16(1), 87-93. doi: 10.1016/j.ejon.2011.04.001

Chaboyer, W. P., \& Patterson, E. (2001). Australian hospital generalist and critical care nurses' perceptions of doctor-nurse collaboration. Nursing and Health Sciences, 3, 73-79.

Chadwick, L. R. (1992). Professional nursing with a new focus: Staff nurse to research coordinator. Journal of Neuroscience Nursing, 24(3), 170 -0172.

Chatfield, D. A. (2008). Role of the specialized neuro intensive care nurse in neuroscience research. European Journal of Anaesthesiology Supplement, 42, 160-163.

Chester, P., Kennedy, E. D., Hynd, S., \& Matthews, D. R. (2007). Clinical research networks in diabetes: The evolving role of the research nurse. European Diabetes Nursing, 4(1), 10-13.

Clarke, A. (2006). Qualitative interviewing: Encountering ethical issues and challenges. Nurse Researcher, 13(4), 19-29.

College of Intensive Care Medicine. (2010a). Guidelines for intensive care units seeking accreditation for training in intensive care medicine (IC-3). Retrieved 10 June, 2010, from http://www.cicm.org.au/cmsfiles/IC-

$3 \% 20$ Guidelines\%20for\%20Intensive \%20Care \%20Units\%20Seeking\%20Accreditat ion\%20for\%20Training\%20in\%20Intensive\%20Care\%20Medicine.pdf

College of Intensive Care Medicine. (2010b). Minimum standards for intensive care units (IC-01). Retrieved 30 November, 2010, from http://www.cicm.org.au/cmsfiles/IC1\%20Minimum\%20Standards\%20for\%20Intensive\%20Care\%20Units.pdf

College of Intensive Care Medicine. (2012a). 'About us' webpage. Retrieved 12 June, 2012, from http://www.cicm.org.au/aboutus.php

College of Intensive Care Medicine. (2012b). Units approved for advanced training. Retrieved 16 March, 2012, from http://www.cicm.org.au/accredunits.php\#NZ 
Coombs, M., \& Ersser, S. J. (2004). Medical hegemony in decision-making -- a barrier to interdisciplinary working in intensive care? Journal of Advanced Nursing, 46(3), 245-252. doi: 10.1111/j.1365-2648.2004.02984.x

Cooper, D. J., Rosenfeld, J. V., Murray, L., Arabi, Y. M., Davies, A. R., D'Urso, P., . . Wolfe, R. (2011). Decompressive craniectomy in diffuse traumatic brain injury. New England Journal of Medicine, 364(16), 1493-1502. doi: doi:10.1056/NEJMoa1102077

Coulson, S., \& Phelan, L. (2000). Clinical research in paediatric oncology and the role of the research nurse in the UK. European Journal of Oncology Nursing, 4(3), 154161.

Covic, A., Kothawala, P., Bernal, M., Robbins, S., Chalian, A., \& Goldsmith, D. (2009). Systematic review of the evidence underlying the association between mineral metabolism disturbances and risk of all-cause mortality, cardiovascular mortality and cardiovascular events in chronic kidney disease. Nephrology Dialysis Transplantation, 24(5), 1506-1523. doi: 10.1093/ndt/gfn613

Crabtree, B. F., \& Miller, W. L. (1992). Doing qualitative research (Vol. 3). Newbury Park, CA: Sage.

Creswell, J. W. (2009). Research design. Qualitative, quantitative and mixed methods approaches (3rd ed.). Thousand Oaks, CA: Sage.

Crotty, M. (1998). The foundations of social research. Crows Nest, Australia: Allen \& Unwin.

Davis, A. M., Hull, S. C., Grady, C., Wilford, B. S., \& Henderson, G. E. (2002). The invisible hand in clinical research: The study coordinator's critical role in human subjects protection. Journal of Law, Medicine \& Ethics, 30, 411-419.

DeSantis, L., \& Ugarriza, D. N. (2000). The concept of theme as used in qualitative nursing research. Western Journal of Nursing Research, 22(3), 351-372.

DiCicco-Bloom, B., \& Crabtree, B. F. (2006). The qualitative research interview. Medical Education, 40(4), 314-321.

District Health Boards/New Zealand Nurses Organisation. (2012). District Health Boards/New Zealand Nurses Organisation Multi-employer collective agreement 2011 - 2015 Retrieved 23 July, 2012, from http://www.nzno.org.nz/dhb

District Health Boards Shared Services. (2012). Job evaluation review committee. Retrieved 23 July, 2012, from http://www.dhbsharedservices.health.nz/site/er/projects/jerc.aspx

Duane, C., Granda, S., Munz, D., \& Cannon, J. (2007). Study coordinators' perceptions of their work experiences. The Monitor(September 2007), 39-41.

Edwards, J. J. (1976). Haematology research nurse in the United States. Nursing Times, 72(5), 194-195. 
Ehrenberger, H. E., \& Lillington, L. (2004). Development of a measure to delineate the clinical trials nursing role. Oncology Nursing Forum, 31(3), E64 - E68.

European Medicines Agency. (2002). Note for guidance on good clinical practice Step 5. Retrieved 10 May, 2012, from http://www.ema.europa.eu/docs/en_GB/document_library/Scientific_guideline/2009 /09/WC500002874.pdf

European Medicines Agency. (2005). Guideline on data monitoring committee. Retrieved 26 April, 2012, from http://www.emea.europa.eu/docs/en_GB/document_library/Scientific_guideline/200 9/09/WC500003635.pdf

Fergus, B. E. B., \& Watts, H. G. H. (1967). The intensive care unit at Tauranga Hospital. The New Zealand Medical Journal, 66(422), 673-678.

Finfer, S., Liu, B., Taylor, C., Bellomo, R., Billot, L., Cook, D., .. . SAFE TRIPS Investigators. (2010). Resuscitation fluid use in critically ill adults: an international cross-sectional study in 391 intensive care units. Critical Care and Resuscitation, 14(5).

Fouka, G., \& Mantzorou, M. (2011). What are the major ethical issues in conducting research? Is there a conflict between the research ethics and the nature of nursing? Health Science Journal, 5(1), 3-14.

Fowler, S. B., \& Stack, K. (2007). Research and the clinical trials coordinator. Journal of Neuroscience Nursing, 39(2), 120-123.

Gillis, A., \& Jackson, W. (2002). Research for nurses: Methods and interpretation. Philadelphia, PA: F.A. Davis.

Gordon, C. (2008). Exploring the new specialty of clinical research nursing (extended version). Nursing Times.net. Retrieved 19 August, 2009 from http://www.nursingtimes.net/exploring-the-new-specialty-of-clinical-researchnursing/1735530.article

Harrison, L., \& Nixon, G. (2002). Nursing activity in general intensive care. Journal of Clinical Nursing, 11(2), 158-167. doi: 10.1046/j.1365-2702.2002.00584.x

Health and Disability Commissioner (2009). Code of Health and Disablilty Services Consumers' Rights. Retrieved 18 November, 2012, from http://www.hdc.org.nz/media/24833/leaflet\%20code\%20of\%20rights.pdf

Health Research Council of New Zealand. (1993). Terms of Reference for Standing Committee on Therapeutic Trials (SCOTT). Retrieved 24 April, 2012, from http://www.hrc.govt.nz/sites/default/files/SCOTT\%20TOR.pdf

Hicks, P. R., \& Mackle, D. M. (2010). Cause of death in intensive care patients within 2 years of discharge from hospital. Critical Care and Resuscitation, 12(2), 78-82.

Hill, G., \& MacArthur, J. (2006). Professional issues associated with the role of the research nurse. Nursing Standard, 20(39), 41-47. 
Intensive Care Clinical Advisory Group. (2005). Intensive care services in New Zealand. A report to the Deputy Director-General, Clinical Services. Retrieved 10 November, 2011, from

http://www.moh.govt.nz/moh.nsf/0/30B1B421D7B627B6CC257036000792F5/\$File /intensivecare.doc

Intensive Care Research Coordinators Interest Group. (2011). Terms of reference version 4 (Vol. 2010, pp. 1-12).

Intensive Care Research Coordinators Interest Group.. (2012). Intensive Care Research Coordinators Interest Group Home page. Retrieved 6 June, 2012, from http://www.anzics.com.au/ctg/ircig

Irvine, D., Sidani, S., \& Hall, L. M. (1998). Finding value in nursing care: A framework for quality improvement and clinical evaluation. Nursing Economic\$, 16(3), 110.

Jeong, I., Kang, H.-S., \& Kim, W.-O. (2007). Clinical research nurses: Roles and qualifications in South Korea. Drug Information Journal, 41(2), 251-256.

Johnson, H. M. (2008). Clinical trials in the intensive care setting: A nursing perspective. Master of Nursing, Otago Polytechnic, Dunedin, NZ.

Jootun, D., McGhee, G., \& Marland, G. R. (2009). Reflexivity: Promoting rigour in qualitative research. Nursing Standard, 23(23), 42-46.

Kenkre, J. E., \& Foxcroft, D. R. (2001). Clinical pathways in research: Clinical research. Nursing Standard, 16(5), 41-44.

Kerman, F. F. (1970). An experimental clinic for narcotic abusers - the nurse as a research coordinator. The Alumnae Magazine, 69(1), 5-7.

Lambert, S. D., \& Loiselle, C. G. (2008). Combining individual interviews and focus groups to enhance data richness. Journal of Advanced Nursing, 62(2), 228-237. doi: 10.1111/j.1365-2648.2007.04559.x

Liamputtong, P. (2009). Qualitative research methods (3rd ed.). South Melbourne, Australia: Oxford University Press Australia and New Zealand.

Lindquist, R., VanWormer, A., Lindgren, B., MacMahon, K., Robiner, W., \& Finkelstein, S. (2011). Time-motion analysis of research nurse activities in a lung transplant home monitoring study. Progress in Transplantation, 21(3), 190-199.

Loh, W. Y., Butow, P. N., Brown, R. F., \& Boyle, F. (2002). Ethical communication in clinical trials. Issues faced by data managers in obtaining informed consent. Cancer, 95(11), 2414-2421.

MacDonald, H. (2007). Relational ethics and advocacy in nursing: Literature review. Journal of Advanced Nursing, 57(2), 119-126. doi: 10.1111/j.13652648.2006.04063.x

McBrien, B. (2008). Evidence-based care: Enhancing the rigour of a qualitative study. British Journal of Nursing, 17(20), 1286-1289. 
Miles, M. B., \& Huberman, A. M. (1994). An expanded sourcebook. Qualitative data analysis (2nd ed.). Thousand Oaks, CA: Sage.

Milne, J., \& Oberle, K. (2005). Enhancing rigor in qualitative description. Journal of Wound, Ostomy \& Continence Nursing November/December, 32(6), 413-420.

Ministry of Health. (2002). Reducing inequalities in health. Retrieved 23 April, 2012, from http://www.health.govt.nz/publication/reducing-inequalities-health

Ministry of Health. (2006). Operational standard for ethics committees. Retrieved 10 November, 2011, from http://www.ethicscommittees.health.govt.nz/moh.nsf/pagescm/6777/\$File/Operation alStandard2006.pdf

Ministry of Health. (2007). Ethical review in New Zealand - history. Retrieved 15 June, 2010, from http://www.ethicscommittees.health.govt.nz/moh.nsf/indexcm/ethicsethicalreview-history?Open\&m_id=3.3

Ministry of Health. (2010a). District health boards. Retrieved 24 April, 2012, from http://www.moh.govt.nz/districthealthboards

Ministry of Health. (2010b). Tatau Kahukura: Maori health chart book 2010. (2nd ed.). Wellington, NZ: Ministry of Health.

Ministry of Health. (2011). Guideline on the regulation of therapeutic products in New Zealand Part 11. Edition 1.1. Retrieved 11 November, 2011, from www.medsafe.govt.nz/regulatory/Guideline/GRTPNZ/Part11.doc

Mori, C., Mullen, N., \& Hill, E. E. (2007). Describing the role of the clinical research nurse. Research Practitioner, 8(6), 220-228.

Morley, A. (2005). Minimum guidelines for intensive care nurse staffing in New Zealand. Retrieved 26 April, 2012, from http://www.nzno.org.nz/LinkClick.aspx?fileticket=Y4SmJPbXvhI\%3d\&tabid=317

Moule, P., \& Goodman, M. (2009). Research in nursing Nursing research: An introduction. Los Angeles, CA: Sage.

Mueller, M.-R. (2001). From delegation to specialization: Nurses and clinical trial coordination. Nursing Inquiry, 8(3), 182-190.

Mueller, M.-R., \& Mamo, L. (2000). Changes in medicine, changes in nursing: Career contingencies and the movement of nurses into clinical trial coordination. Sociological Perspectives, 43(4), s43-57.

Mueller, M.-R., \& Mamo, L. (2002). The nurse clinical trial coordinator: Benefits and drawbacks of the role. Research and Theory for Nursing Practice: An International Journal, 16(1), 33-42.

Mullin, S. M., Warwick, S., Akers, M., Beecher, P., Helminger, K., Moses, B., . . Wettach, R. (1984). An acute intervention trial: The research nurse coordinator's role. Controlled Clinical Trials, 5(2), 141-156. doi: 10.1016/0197-2456(84)90120-x 
Murray, L. (2011). Research 101. Paper presented at the Research Coordinator Workshop.

Nagel, K., Gender, J., \& Bonner, A. (2010). Delineating the role of a cohort of clinical research nurses in a pediatric cooperative clinical trials group. Oncology Nursing Forum, 37(3), E180.

National Ethics Advisory Committee. (2006). Ethical guidelines for observational studies: Observational research, audits and related activities. Retrieved 6 June, 2012, from http://www.neac.health.govt.nz/moh.nsf/pagescm/520/\$File/ethicalguidelines.pdf

New Zealand Health and Disability Ethics Committees. (2010). Reporting of serious adverse events. Retrieved 3 June 2012, from http://www.ethicscommittees.health.govt.nz/moh.nsf/indexcm/ethics-formsreportingsaes?Open\&m_id=5.4

New Zealand Health and Disability Ethics Committees. (2012). Standard operating procedures for health and disability ethics committees. Wellington, NZ: Ministry of Health.

New Zealand Health and Disability Ethics Committees. (n.d.). Multi-region Ethics Committee terms of reference. Retrieved 24 April, 2012, from http://www.ethicscommittees.health.govt.nz/moh.nsf/indexcm/ethics-aboutmulti?Open\&m_id=2.1\#tor

New Zealand Government (2000). New Zealand Public Health and Disability Act (2000). Retrieved 23 April, 2012 from http://www.legislation.govt.nz/act/public/2000/0091/latest/DLM80051.html

New Zealand Nurses Organisation. (2009). Home page. Retrieved 29 July, 2012, from http://www.nzno.org.nz/

NICE Study Investigators. (2009). Intensive versus conventional glucose control in critically ill patients. New England Journal of Medicine, 360(13), 1283-1297. doi: doi:10.1056/NEJMoa0810625

Nursing Council of New Zealand. (2011). Guidelines for cultural safety, the Treaty of Waitangi and Maori health in nursing education and practice. Wellington, NZ.

Nursing Council of New Zealand. (2012). Code of conduct for nurses. Retrieved 30 July, 2012, from http://www.nursingcouncil.org.nz/download/283/coc-web.pdf

NZNO Critical Care Nurses' Section. (2011). The role of the New Zealand critical care clinical research nurse. Retrieved 26 April, 2012, from http://www.nzno.org.nz/LinkClick.aspx?fileticket=i4EKBuKXFtY\%3d\&tabid=317

Opie, A. (1999). Knowledge-based teamwork. In P. Davis \& K. Dew (Eds.), Health and society in Aotearoa New Zealand (pp. 181 - 197). Auckland, NZ: Oxford University Press.

Oxford University Press. (n.d.). Oxford Dictionaries (on-line version). Retrieved 23 July, 2012, from http://oxforddictionaries.com/definition/english/role?q=role 
Patton, M. Q. (2002). Qualitative research and evaluation methods (3rd ed.). Thousand Oaks, CA: Sage.

Plummer-D'Amato, P. (2008). Focus group methodology part 1: Considerations for design. International Journal of Therapy \& Rehabilitation, 15(2), 69-73.

Poston, R. D., \& Buescher, C. R. (2010). The essential role of the clinical research nurse. Urologic Nursing, 30(1), 55-63, 77.

Project Management Institute. (2008). A guide to the project management body of knowledge (PMBOK guide). PA: Project Management Institute.

Raja-Jones, H. (2002). Role boundaries - research nurse or clinical nurse specialist? Journal of Clinical Nursing, 11, 415-420.

Reid, P. (1999). Te pupuri i te ao o te tangata whenua. In K. Dew \& P. Davis (Eds.), Health and society in Aotearoa New Zealand (pp. 51 - 62). Auckland, New Zealand: Oxford University Press.

Rickard, C. M., Roberts, B. L., Foote, J., \& McGrail, M. R. (2006). Intensive care research coordinators: Who are they and what do they do? Results of a binational survey. Dimensions of Critical Care Nursing, 25(5), 234.

Rickard, C. M., Roberts, B. L., Foote, J., \& McGrail, M. R. (2007). Job satisfaction and importance for intensive care unit research coordinators: Results from binational survey. Journal of Clinical Nursing, 16(9), 1640.

Rico-Villademoros, F., Hernando, T., Sanz, J.-L., Lopez-Alonso, A., Salamanca, O., Camps, C., \& Rosell, R. (2004). The role of the clinical research coordinator - data manager - in oncology clinical trials. BMC Medical Research Methodology, 4(6), 6-15.

Rischbieth, A., \& Blythe, D. (2005). Ethics handbook for researchers (1st ed.). Melbourne, Australia: The Australian and New Zealand Intensive Care Society Clinical Trials Group.

Roberts, B. L., Eastwood, G. M., Raunow, H., Howe, B., \& Rickard, C. M. (2011a). The intensive care research coordinator position in Australia and New Zealand: Selfperception of professional development priorities and "best" and "worst" aspects of the position. A cross-sectional web-based study. Intensive \& Critical Care Nursing, 27(3), 129-137. doi: 10.1016/j.iccn.2011.02.002

Roberts, B. L., Eastwood, G. M., Raunow, H., Howe, B., \& Rickard, C. M. (2011b). Intensive care research coordinators in Australia and New Zealand: A crosssectional survey of demographics, responsibilities, job satisfaction and importance. Australian Critical Care, 24(4), 259-268.

Roberts, B. L., Rickard, C. M., Foote, J., \& McGrail, M. R. (2006). The best and worst aspects of the ICU research coordinator role. Nursing in Critical Care, 11(3), 128.

Ryan, F., Coughlan, M., \& Cronin, P. (2009). Interviewing in qualitative research: The oneto-one interview. International Journal of Therapy \& Rehabilitation, 16(6), 309-314. 
Sandelowski, M. (1993). Rigor or rigor mortis: The problem of rigor in qualitative research revisited. Advances in Nursing Science, 16(2), 1-8.

Sandelowski, M. (1994). The use of quotes in qualitative research. Research in Nursing \& Health, 17(6), 479-482.

Sandelowski, M. (1995). Focus on qualitative methods. Qualitative analysis: What it is and how to begin. Research in Nursing \& Health, 18(4), 371-375.

Sandelowski, M. (2000). Focus on research methods. Whatever happened to qualitative description? Research in Nursing \& Health, 23(4), 334-340.

Sandelowski, M. (2010). What's in a name? Qualitative description revisited. Research in Nursing \& Health, 33(1), 77-84. doi: 10.1002/nur.20362

Silverman, D., \& Marvasti, A. (2008). Doing qualitative research. Thousand Oaks, CA: Sage.

Smith, M., \& Erwin, J. (2007). Role and responsibility charting. Retrieved 31 March, 2012, from http://alliancebestpractice.co.uk/downloaddoc/RACI\%20Explanation.pdf

Snelgrove, S., \& Hughes, D. (2000). Interprofessional relations between doctors and nurses: perspectives from South Wales. Journal of Advanced Nursing, 31(3), 661-667. doi: 10.1046/j.1365-2648.2000.01321.x

Spilsbury, K., Petherick, E., Cullum, N., Nelson, A., Nixon, J., \& Mason, S. (2008). The role and potential contribution of clinical research nurses to clinical trials. Journal of Clinical Nursing, 17(4), 549-557.

Stake, R. E. (1995). The art of case study research. Thousand Oaks, CA: Sage.

Stephens-Lloyd, A. (2004). The extended role of the clinical research nurse: Building an evidence base for practice. Nursing Times Research, 9(1), 18-27.

Tellis, W. (1997). Application of a case study methodology. The Qualitative Report, 3(3).

The ANZIC Influenza Investigators. (2009). Critical care services and 2009 H1N1 influenza in Australia and New Zealand. New England Journal of Medicine, 361, 1-10.

The Blood Observational Study Investigators on behalf of the ANZICS-Clinical Trials Group. (2010). Transfusion practice and guidelines in Australian and New Zealand intensive care untis. Intensive Care Medicine, 36(7), 1138-1146. doi: $10.1007 / \mathrm{s} 00134-010-1867-8$

The CHEST Management Committee. (2011). The crystalloid versus hydroxyethyl starch trial: Protocol for a multi-centre randomised controlled trial of fluid resuscitation with $6 \%$ hydroxyethyl starch (130/0.4) compared to $0.9 \%$ sodium chloride (saline) in intensive care patients on mortality. Intensive Care Medicine, 37(5), 816-823. doi: $10.1007 / \mathrm{s} 00134-010-2117-9$

Thomas, D. R. (2006). A general inductive approach for analyzing qualitative evaluation data. American Journal of Evaluation, 27(2), 237-246. 
Trubuhovich, R. V., \& Judson, J. A. (2001). Intensive care in New Zealand. A History of the New Zealand region of ANZICS: The Department of Critical Care Medicine, Auckland Hospital.

United Kingdom Clinical Research Collaboration. (2007). Developing the best research professionals. Retrieved 10 June, 2010, from

http://www.ukcrc.org/publications/reports/

University of Otago. (n.s.). Study Clinical Research at Otago. Retrieved 10 November, 2012, from http://www.otago.ac.nz/courses/subjects/cres.html

Victoria University of Wellington. (n.s.). Postgraduate Diploma of Clinical Research . Retrieved 30 July, 2012, from http://www.victoria.ac.nz/sbs/study/postgraduatestudy/clinical-res\#PGDipCR

von Elm, E., Altman, D. G., Egger, M., Pocock, S. J., Gøtzsche, P. C., \& Vandenbroucke, J. P. (2007). The strengthening the reporting of observational studies in epidemiology (STROBE) statement: Guidelines for reporting observational studies. The Lancet, 370(9596), 1453.

Walker, L. (2009a). Recognising clinical research nurses. Kai Tiaki Nursing New Zealand, 15(11), 26.

Walker, L. (2009b). Research nursing issues. Paper presented at the New Zealand ICU research nurses' workshop, Wellington.

Walker, L. (2010). Clinical research nursing. Paper presented at the NZ Association of Clinical Research conference, Auckland.

Walker, L., Clendon, J., McKinlay, E., Harker, D., Gilmour, C., Anderson, A., . . Shaw, B. (2010). Draft New Zealand Competency Framework for Clinical Research Nurses, Version 6, August 2010.

Waller, J. (2002). An insight into the role of the clinical trials coordinator. Australian Nursing Journal, 10(6), 17-18.

Watmough, S., Flynn, M., Wright, A., \& Fry, K. (2010). Research nurse or nurse researcher? British Journal of Cardiac Nursing, 5(8), 396-399.

Whiting, L. S. (2008). Semi-structured interviews: Guidance for novice researchers. Nursing Standard, 22(23), 35-40.

Williams, M. (2008). The principles of project management. VIC, Australia: Sitepoint.

World Medical Association. (1964). Declaration of Helsinki . Retrieved 6 June, 2012, from http://www.wma.net/en/30publications/10policies/b3/17c.pdf 